\title{
Metabolic Syndrome in Childhood Cancer Survivors
}

Metabool syndroom in overlevenden van kinderkanker

Marjolein van Waas 
Publication of this thesis was financially supported by:

Stichting Kinderoncologisch Centrum Rotterdam (SKOCR)

Sanofi B.V.

Novartis Oncology Pharma B.V.

Ipsen Farmaceutica B.V.

Cover: "Tree of possibilities" by Irene van Waas-Korpershoek

Lay-out: Legatron Electronic Publishing, Rotterdam

Printing: Ipskamp Drukkers BV, Enschede

ISBN/EAN: 9789461913807

2012 (CM. van Waas

No part of this thesis may be reproduced, stored in a retrieval system or transmitted in any form or by any means, without written permission of the author or, when appropriate, of the publishers of the publications. 


\section{Metabolic Syndrome in Childhood Cancer Survivors}

Metabool syndroom in overlevenden van kinderkanker

\section{Proefschrift}

ter verkrijging van de graad van doctor aan de

Erasmus Universiteit Rotterdam

op gezag van de

rector magnificus

Prof.dr. H.G. Schmidt

en volgens besluit van het College voor Promoties.

De openbare verdediging zal plaatsvinden op woensdag 19 september 2012 om 13.30 uur

door

Marjolein van Waas

geboren te Vlaardingen

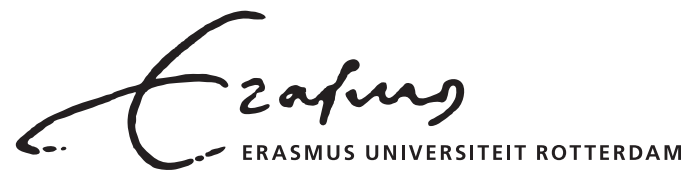




\section{Promotiecommissie:}

Promotor: $\quad$ Prof.dr. R. Pieters

Overige leden: $\quad$ Prof.dr. A.J. van der Lelij

Prof.dr. A.P.N. Themmen

Prof.dr. J.J. van Binsbergen

Copromotoren: Dr. M.M. van den Heuvel-Eibrink

Dr. S.J.C.M.M. Neggers 


\section{Contents}

Chapter 1 General introduction and aims of the thesis

Chapter 2 The metabolic syndrome in adult survivors of childhood cancer, a review J Pediatr Hematol Oncol 2010; 32:171-9

Chapter 3 Components of the metabolic syndrome in 500 adult long-term survivors of childhood cancer Ann Oncol 2010; 21:1121-6

Chapter 4 Endocrine late sequelae in long-term survivors of childhood non-Hodgkin lymphoma

Ann Oncol 2011;23:1626-32

Chapter 5 Adrenal function in adult long-term survivors of nephroblastoma and neuroblastoma

Eur J Cancer 2012;48:1159-66

Chapter 6 Daily life physical activity in adult long-term nephroblastoma and neuroblastoma survivors

submitted

Chapter 7 Abdominal irradiation: An important determinant of metabolic syndrome after nephroblastoma and neuroblastoma

Submitted

Chapter 8 Cardiovascular risk factors in adult very long-term survivors of 109 nephroblastoma and neuroblastoma submitted

Chapter 9 Treatment factors rather than genetic variation determine metabolic syndrome in childhood cancer survivors

submitted 
Nederlandse samenvatting

Affiliations co-authors

List of abbreviations

Dankwoord

Curriculum vitae

175

List of publications

PhD portfolio 



\subsection{Childhood cancer}

Over 200,000 children under the age of fifteen are diagnosed with cancer worldwide every year. ${ }^{1,2}$ Cancer is the second most common cause of death among children between the ages of 1 and 14 years in developed countries, surpassed only by accidents. ${ }^{1,3}$ Nearly one third of the cancers diagnosed in children are leukemias (particularly acute lymphoblastic leukemia (ALL)), followed by cancer of the brain or central nervous system (21\%), soft tissue sarcomas (including neuroblastoma (7\%) and rhabdomyosarcoma (3\%)), renal (nephroblastoma) tumors (5\%), and non-Hodgkin lymphoma $(4 \%) .^{3}$

\section{Survival}

Childhood cancer survival has increased significantly over the past few decades (Figure 1). Especially the advent of combination chemotherapy in the late 1960s and 1970s brought about large increases in survival for many childhood cancers. Furthermore, improved surgery, radiotherapy, stem cell transplantation, supportive care and better stratification of therapy regimens played an important role. ${ }^{4,5}$ Population-based statistics show the probability of fiveyear survival of cancer in those under the age of 20 in the United States to be $80 \% .{ }^{6}$ This success in therapy translates into a growing and aging population of long-term survivors. To date, 1 out of 640 young adults in the United States is a childhood cancer survivor. ${ }^{7}$ In the Netherlands, currently 7000 adults are long-term childhood cancer survivors.

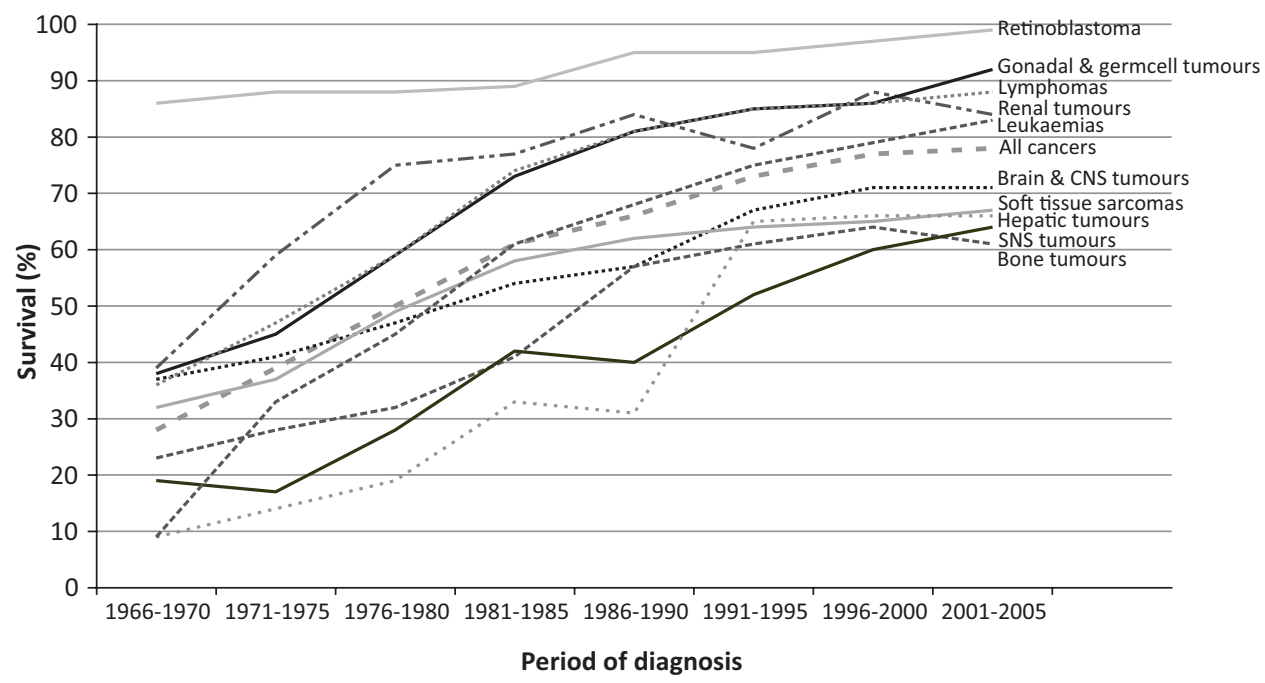

Figure 1. Five-year survival by cancer type in children aged $0-14$ years.

Stiller C, ed. Oxford University Press; 2007. 


\section{Mortality and morbidity in childhood cancer survivors}

With an ever increasing absolute number of long-term survivors, research has focussed on mortality and morbidity in childhood cancer survivors. In the past few decades, it has become evident that cure alone is not enough.

An overall standardized mortality ratio (SMR) of 8.4 in childhood cancer survivors was reported by Mertens et al. ${ }^{8}$ Increases in cause-specific mortality were seen for deaths due to subsequent malignancy $(S M R=15.2)$ and cardiac $(S M R=7.0)$, pulmonary (SMR=8.8), and other medical causes $(S M R=2.6)$. At 20 years of follow-up, the death rate due to a subsequent malignancy exceeded that due to all other causes. Deaths from pulmonary, cardiac, and other causes were relatively low during the 5- to 15-year interval, but increases were observed 15 - 30 years after diagnosis of the original cancer. ${ }^{8}$ While mortality due to recurrence or progression seems to reach a plateau, mortality due to nonreccurrence and nonexternal causes continues to increase, and even 30 years after diagnosis does no seem to plateau (Figure 2).

Besides increased mortality rates survivors of childhood cancer have an increased risk for developing chronic health conditions. More than $60 \%$ of survivors has at least one chronic condition and $27.5 \%$ has a severe or life-threatening condition. ${ }^{9}$

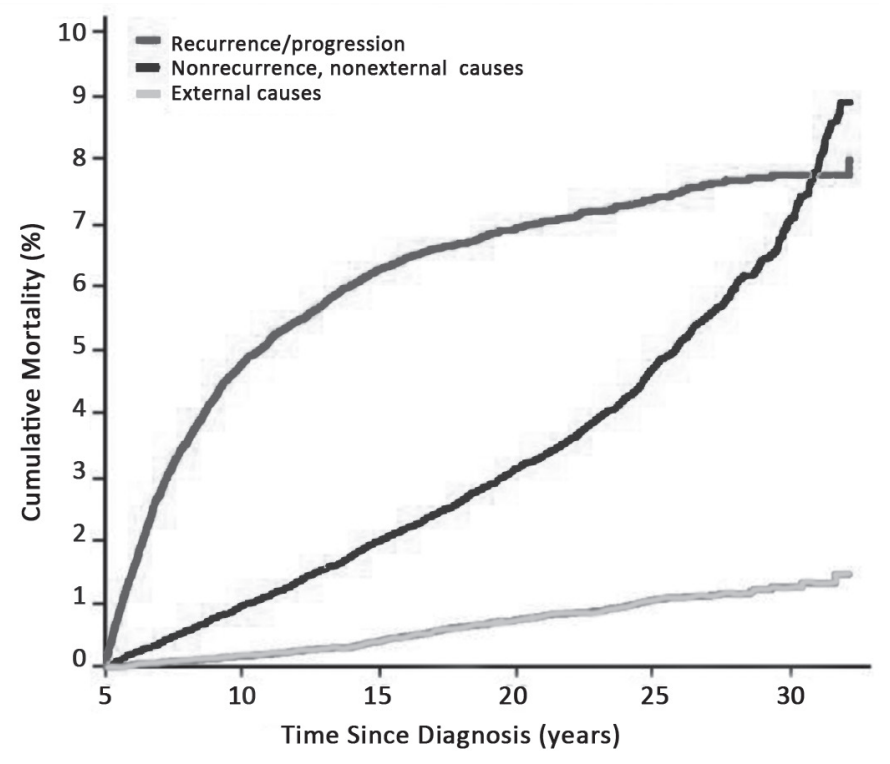

Figure 2. Cumulative cause-specific mortality. Armstrong G T et al. J Clin Oncol 2009;27:2328-2338

In particular the risk for second cancers, cardiovascular disease, renal dysfunction, severe muscoskeletal problems and endocrinopathies is high. Treatment combinations including chest, abdominal or pelvic irradiation have been associated with an increased risk for severe or life- 
threatening conditions, and female survivors had a greater risk of diminished health status than males. ${ }^{9}$ Similar findings were reported by a Dutch study: almost $75 \%$ of survivors had one or more adverse events, and $24.6 \%$ had five or more adverse events. Furthermore, $40 \%$ of survivors had at least one severe or life-threatening or disabling adverse event. A high or severe burden of adverse events was observed in $55 \%$ of survivors who received radiotherapy only and $15 \%$ of survivors treated with chemotherapy only, compared with $25 \%$ of survivors who had surgery only. ${ }^{10}$

\subsection{Metabolic syndrome}

\section{Prevalence and definitions}

Metabolic syndome is a cluster of adiposity, insulin resistance, hypertension and dyslipidemia. Furthermore, proinflammatory and prothrombotic factors are associated with the metabolic syndrome (Figure 3). It is estimated that $20-25 \%$ of the world's adult population have this syndrome and are, as a result, twice as likely to die from and three times more likely to have a heart attack or stroke compared to people without metabolic syndrome. In addition, these people are five times more likely to develop type 2 diabetes and people with diabetes are three times more likely to develop cardiovascular diseases (Figure 4). ${ }^{11}$ Prevalence of the metabolic syndrome in the Netherlands is $14 \%$ : in male subjects aged $28-39$ years it is $12 \%$ in and in females $5 \% .^{12}$

The three most current definitions of metabolic syndrome, are those created by the National Cholesterol Education Program - Third Adult Treatment Panel (NCEP/ATPIII), ${ }^{14}$ the World Health Organization (WHO), ${ }^{15}$ and the International Diabetes Foundation (IDF), ${ }^{11}$ which provide useful guidelines to identify those individuals at increased risk for development of type 2 diabetes, atherosclerotic cardiovascular diseases, and cardiovascular death. Although the utility of establishing criteria for a syndrome is based on the principle that the individual components either act synergistically to amplify risk or cluster together, conferring additive risk, the components comprising the metabolic syndrome are also independent risk factors for the development of atherosclerotic cardiovascular disease. ${ }^{15}$

\section{Potential molecular mechanisms involved in development of the metabolic syndrome} Increased adiposity, particularly increased visceral adiposity, results from a centrally regulated imbalance in energy intake versus expenditure. Secondary effects of adiposity include increased circulating free fatty acids (FFAs), in part resulting from a net increase in lipolysis; reduced adiponectin; a potential increase in insulin resistance factors; and increased proinflammatory mediators such as tumour necrosis factor (TNF)-alpha. Increased lipid flux into peripheral tissues can result in accumulation of triglycerides and long-chain fatty acyl-CoA esters (steatosis), which mediate insulin resistance and pancreatic beta cell "lipotoxicity". Insulin resistance in the liver and muscle leads to hyperinsulinemia, which can, in turn, augment adipose tissue accretion and exacerbate tissue resistance to insulin (Figure 3 ). ${ }^{15}$ 


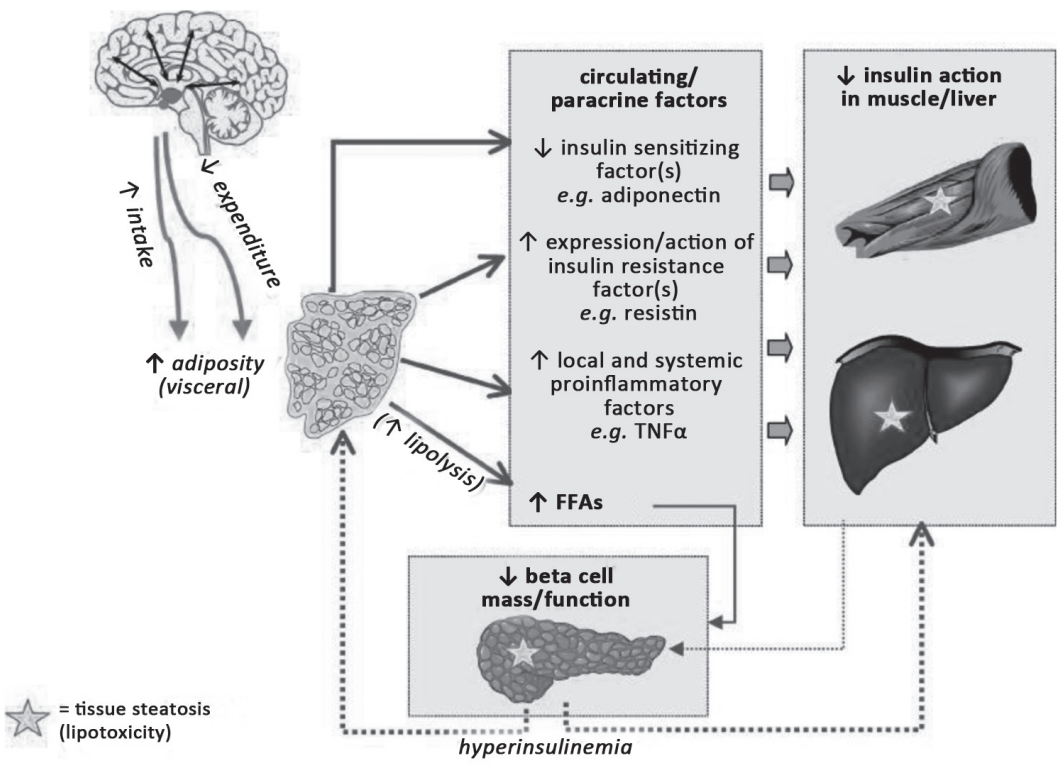

Figure 3. Pathofysiology of the metabolic syndrome.

Moller DE, Kaufman KD. Annu Rev Med 2005;56:45-62.

\section{Treatment and prevention}

The primary approach to the treatment and prevention of metabolic syndrome is lifestyle change. With a substantial component of metabolic syndrome attributed to unhealthy diet and sedentary lifestyle, improvements in these areas will likewise prevent clinical manifestations of this disease such as hypertension, dyslipidemia, insulin sensitivity, and glucose tolerance. ${ }^{15}$ The secondary approach for treatment is to treat the individual clinical manifestations. Since metabolic syndrome is a cluster of symptoms that presents uniquely between individuals, treatment with medication is handled similarly, unique to each individual. ${ }^{15}$

There is currently no drug therapy to treat metabolic syndrome as a whole disease, however extensive research continues to be published yearly. ${ }^{15}$

\subsection{Metabolic syndrome in childhood cancer survivors}

There is a growing body of evidence indicating that pediatric cancer survivors are at a greater risk of developing metabolic syndrome or metabolic syndrome component traits than are members of the general population. ${ }^{16-21}$ In Chapter two, literature on the (components of) the metabolic syndrome in childhood cancer survivors is summarized. 
In the normal population, metabolic syndrome is known to be determined by genetic makeup and environmental factors, including maternal influences and lifestyle factors (Figure 4). In survivors of childhood cancer, treatment or disease, but also the occurrence of other late effects, might result in increased prevalence of the metabolic syndrome and, consequently, an increased risk for type 2 diabetes and cardiovascular disease.

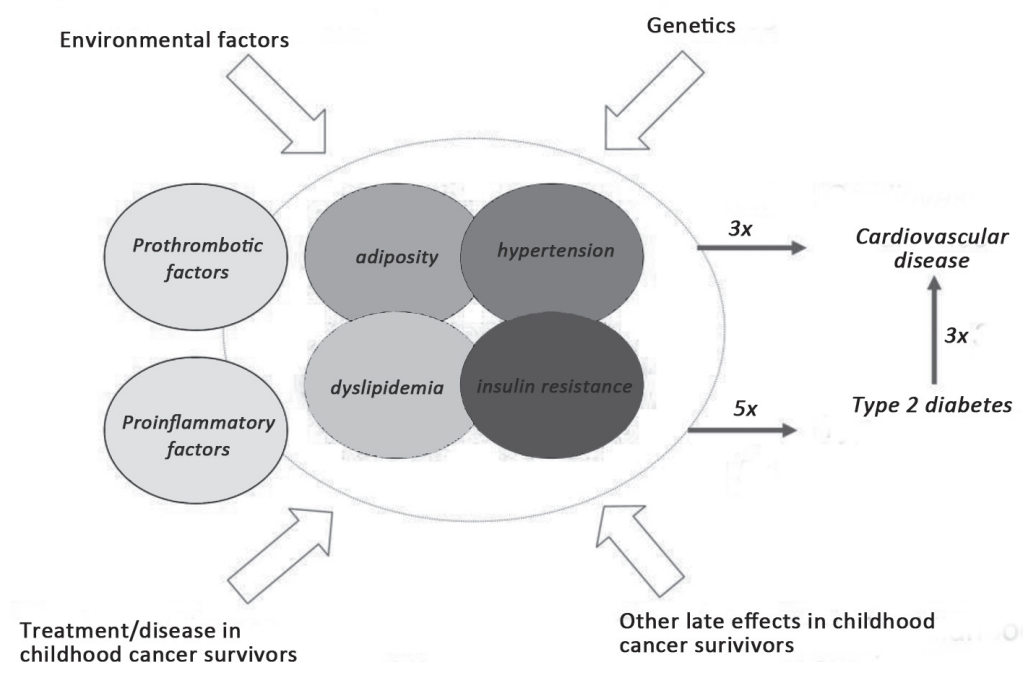

Figure 4. Metabolic syndrome in childhood cancer survivors.

\subsection{Aims of the study}

The general aim of the research presented in this thesis is to assess the (components of the) metabolic syndrome in childhood cancer survivors. Specific aims are:

1. To determine the frequency of the metabolic syndrome in adult survivors of childhood cancer.

2. To identify which risk factors, especially treatment factors, increase the risk for metabolic syndrome after childhood cancer.

3. To identify additional cardiovascular risk factors associated with the metabolic syndrome in childhood cancer survivors.

4. To identify the influence of genetic variation on the development of the metabolic syndrome in childhood cancer survivors. 


\section{Outline of this thesis}

Chapter 1 covers the general introduction of childhood cancer, childhood cancer survival and the metabolic syndrome.

In Chapter 2, a review of the literature concerning metabolic syndrome and its individual components in childhood cancer survivors is presented.

In Chapter 3, the prevalence of the metabolic syndrome in our single centre cohort is described, with a special focus on survivors of ALL.

In Chapter 4, we focussed on endocrine late effects, including adiposity and body compositon, in survivors of non-Hodgkin lymphoma.

In Chapter 5, we assessed adrenal function and its influence on metabolic syndrome in survivors of nephroblastoma and neuroblastoma.

In Chapter 6, we studied physical activity in nephroblastoma and neuroblastoma survivors.

In Chapter 7, we assessed the metabolic syndrome and focussed on abdominally irradiated nephroblastoma and neuroblastoma survivors.

In Chapter 8, we assessed proinflammatory factors, prothrombotic factors and vascular parameters in nephroblastoma and neuroblastoma survivors.

In Chapter 9, we assessed the influence of genetic variation on the development of the metabolic syndrome in childhood cancer survivors.

Chapter $\mathbf{1 0}$ covers the general discussion and conclusions. 


\section{References}

1. Kaatsch P. Epidemiology of childhood cancer. Cancer Treat Rev 2010;36:277-85.

2. Terracini B. Epidemiology of childhood cancer. Environ Health 2011;10 Suppl 1:S8.

3. Jemal A, Siegel R, Xu J, Ward E. Cancer statistics, 2010. CA Cancer J Clin 2010;60:277-300.

4. Meadows AT. Pediatric cancer survivors: Past history and future challenges. Curr Probl Cancer 2003;27:112-26.

5. van den Heuvel-Eibrink MM, Tissing WJE. Compendium kindergeneeskunde. 4th ed. Houten: Bohn Stafleu van Loghum; 2011.

6. Ries LAG, Eisner MP, Kosary CL, et al. SEER Cancer Statistics Review, 1975-2002, National Cancer Institute, Bethesda, MD. 2005.

7. Hewitt M, Weiner SL, Simone JV, editors. National Research Council. Childhood Cancer Survivorship: Improving Care and Quality of Life. . Washington, DC: National Academies Press 2003.

8. Mertens AC, Liu Q, Neglia JP, et al. Cause-specific late mortality among 5-year survivors of childhood cancer: the Childhood Cancer Survivor Study. J Natl Cancer Inst 2008;100:1368-79.

9. Oeffinger KC, Mertens AC, Sklar CA, et al. Chronic health conditions in adult survivors of childhood cancer. N Engl J Med 2006;355:1572-82.

10. Geenen MM, Cardous-Ubbink MC, Kremer LC, et al. Medical assessment of adverse health outcomes in long-term survivors of childhood cancer. JAMA 2007;297:2705-15.

11. Alberti KG, Zimmet P, Shaw J. Metabolic syndrome--a new world-wide definition. A Consensus Statement from the International Diabetes Federation. Diabet Med 2006;23:469-80.

12. Bos $M B$, de Vries $J H$, Wolffenbuttel BH, Verhagen $H$, Hillege JL, Feskens EJ. [The prevalence of the metabolic syndrome in the Netherlands: increased risk of cardiovascular diseases and diabetes mellitus type 2 in one quarter of persons under 60].

De prevalentie van het metabool syndroom in Nederland: verhoogd risico op hart- en vaatziekten en diabetes mellitus type 2 bij een kwart van de personen jonger dan 60 jaar. Ned Tijdschr Geneeskd 2007;151:2382-8.

13. Expert Panel on Detection E, Treatment of High Blood Cholesterol in A. Executive Summary of The Third Report of The National Cholesterol Education Program (NCEP) Expert Panel on Detection, Evaluation, And Treatment of High Blood Cholesterol In Adults (Adult Treatment Panel III). JAMA 2001;285:248697.

14. Alberti KG, Zimmet PZ. Definition, diagnosis and classification of diabetes mellitus and its complications. Part 1: diagnosis and classification of diabetes mellitus provisional report of a WHO consultation. Diabet Med 1998;15:539-53.

15. Moller DE, Kaufman KD. Metabolic syndrome: a clinical and molecular perspective. Annu Rev Med 2005;56:45-62.

16. Trimis G, Moschovi M, Papassotiriou I, Chrousos G, Tzortzatou-Stathopoulou F. Early indicators of dysmetabolic syndrome in young survivors of acute lymphoblastic leukemia in childhood as a target for preventing disease. J Pediatr Hematol Oncol 2007;29:309-14.

17. Oeffinger KC. Are survivors of acute lymphoblastic leukemia (ALL) at increased risk of cardiovascular disease? Pediatr Blood Cancer 2008;50:462-7.

18. Nuver J, Smit AJ, Postma A, Sleijfer DT, Gietema JA. The metabolic syndrome in long-term cancer survivors, an important target for secondary preventive measures. Cancer Treat Rev 2002;28:195-214.

19. Meacham LR, Chow EJ, Ness KK, et al. Cardiovascular risk factors in adult survivors of pediatric cancer a report from the childhood cancer survivor study. Cancer Epidemiol Biomarkers Prev 2010;19:170-81.

20. Gurney JG, Ness KK, Sibley SD, et al. Metabolic syndrome and growth hormone deficiency in adult survivors of childhood acute lymphoblastic leukemia. Cancer 2006;107:1303-12.

21. Follin C, Thilen U, Ahren B, Erfurth EM. Improvement in cardiac systolic function and reduced prevalence of metabolic syndrome after two years of growth hormone (GH) treatment in GH-deficient adult survivors of childhood-onset acute lymphoblastic leukemia. J Clin Endocrinol Metab 2006;91:1872-5. 


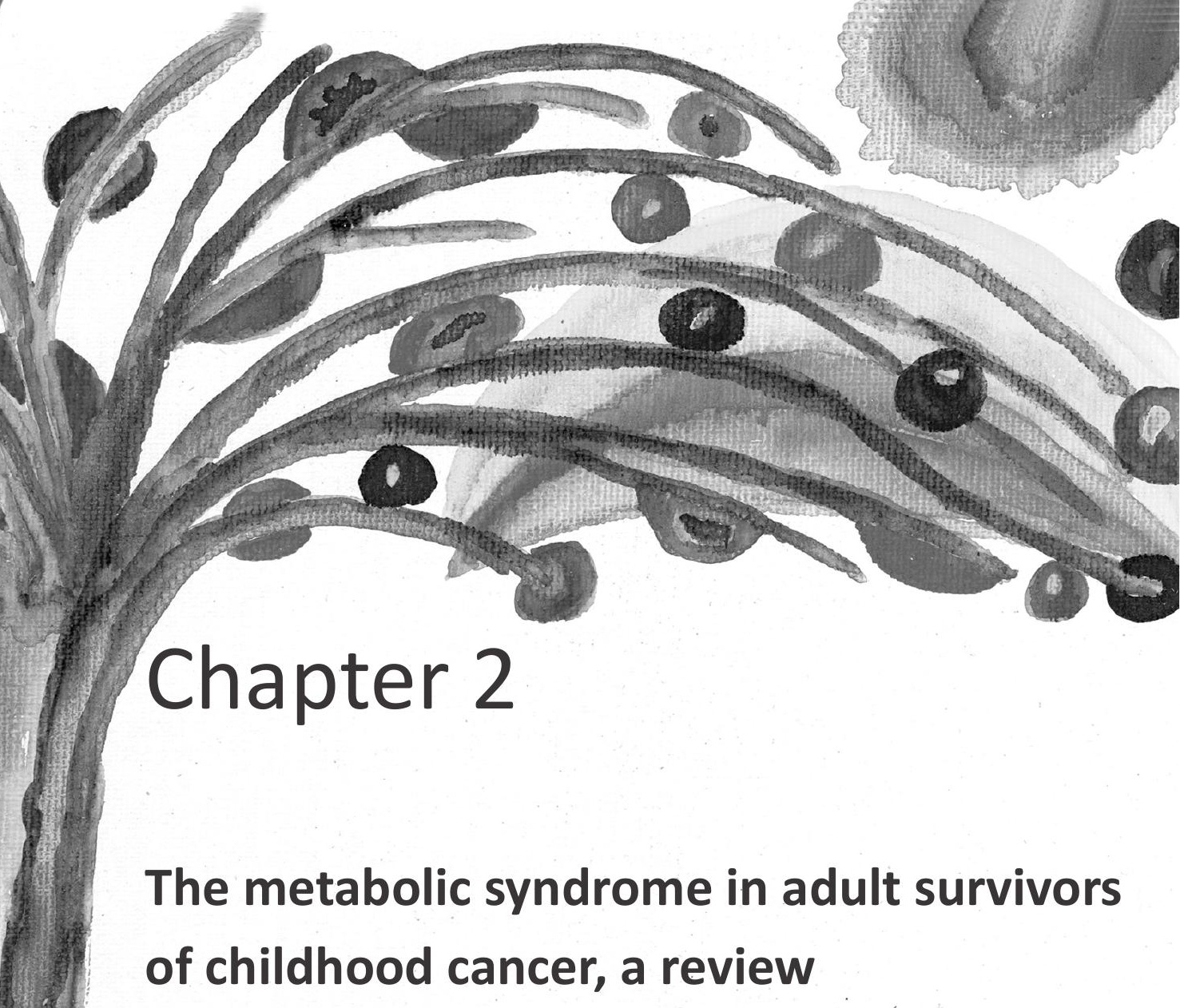

Marjolein van Waas ${ }^{1}$, Sebastian J.C.M.M. Neggers ${ }^{1,2}$, Aart-Jan van der Lely ${ }^{2}$, Rob Pieters ${ }^{1}$, Marry M. van den Heuvel-Eibrink ${ }^{1}$

'Department of Pediatric Oncology/Hematology, Erasmus MC-Sophia Children's Hospital, Rotterdam, the Netherlands

${ }^{2}$ Department of Medicine, Section Endocrinology, Erasmus University Medical Centre, Rotterdam, the Netherlands

J Pediatr Hematol Oncol 2010; 32:171-9 


\begin{abstract}
The number of adult survivors of childhood cancer in the general population is increasing. Because reports on the prevalence of the metabolic syndrome in adult survivors of childhood cancer are scarce, we reviewed the available literature on the components of the metabolic syndrome in adult survivors of childhood cancer. Although there is a lack of studies estimating the prevalence of metabolic syndrome directly, especially prevalence of insulin resistance, obesity and dyslipidemia is increased in certain groups. Therefore, adult survivors of childhood cancer are at increased risk of developing cerebro- and cardiovascular diseases. Accordingly, it is important to identify the predisposing factors of the metabolic syndrome in cohorts of survivors, in order to introduce medical interventions and to subsequently decrease the risk of cerebroand cardiovascular events.
\end{abstract}




\section{Introduction}

\section{Late effects in survivors of childhood cancer}

During the last decades, survival rates of childhood cancer have increased considerably, i.e. from $5-30 \%$ in the early seventies, to currently approximately $80 \%$. This can be explained by the development of (combinations of) effective chemotherapy, surgery, radiotherapy, stem cell transplantation regimens and better supportive care regimens with an optimized stratification of therapy. These improved survival rates result in an ongoing increasing number of survivors. ${ }^{1}$ Some of these survivors are now 30 or even more years after treatment. Therefore, survivors of childhood cancer gain increased attention to identify late side effects of therapy. Various studies investigated the risk of second malignancies, cardiac problems, pulmonary complications, impaired fertility, problems of offspring, osteoporosis and obesity in survivors of childhood cancer. ${ }^{2-7}$ Among others, a standardized mortality ratio of 9.7 for circulatory diseases has been found in survivors of childhood cancer. ${ }^{8}$ Furthermore, components of the metabolic syndrome, such as obesity, dyslipidemia, hypertension, impaired glucose tolerance and insulin resistance have been described in long-term survivors of cancer. ${ }^{3,4,9,10}$ Accordingly, it is important to identify the components of the metabolic syndrome in childhood cancer survivors. To estimate the impact of the metabolic syndrome in adult survivors of childhood cancer, we reviewed the available literature on the metabolic syndrome, and on insulin resistance, obesity, dyslipidemia and hypertension, i.e. the components of the metabolic syndrome, in adult survivors of childhood cancer.

\section{Metabolic syndrome in the normal population \\ Definitions}

The metabolic syndrome was first described by Reaven in $1988 .{ }^{11} \mathrm{He}$ found a clustering of symptoms in patients and called this Syndrome $X$. The syndrome included insulin resistance, hyperinsulinemia, glucose intolerance, elevated levels of triglycerides, reduced high-density lipoprotein-cholesterol (HDL-C) levels and arterial hypertension. It was suggested that this could be an important risk factor for developing cardiovascular diseases and type 2 diabetes mellitus. Since then, many definitions of the metabolic syndrome, with slightly different characteristics, were proposed by different organizations. Cut-offs and threshold values for the various individual components are slightly different (Table 1). ${ }^{12-17}$

The most commonly used definitions are those of the WHO and the NCEP/ATPIII. The WHO definition was meant to facilitate research on the metabolic syndrome and intends to improve comparability between studies, rather than serve as a strict definition. Others criticized the inclusion of microalbuminuria and and the use of waist/hip ratio to assess obesity. The euglycemic clamp, which is the golden standard to measure insulin resistance, is not easy to use in a clinical and/or epidemiologic research setting. The NCEP definition was designed for clinical purposes and does not require at least one specific component to be present, in contrast to the WHO 
definition, which needs insulin resistance, increased fasting plasma glucose or impaired glucose tolerance to be present.

\section{Pathogenesis of metabolic syndrome}

The pathogenesis of the metabolic syndrome is not fully elucidated. However, two factors are supposed to be the key factors: insulin resistance and abdominal obesity. Factors that predispose development of the metabolic syndrome are genetic variation, a proinflammatory state, a prothrombic state, hormonal deregulation, physical inactivity and aging. It is known that central obesity can cause insulin resistance, and vice versa, insulin resistance modifies adipose tissue responses to insulin and thereby recapitulates the obese state..$^{18}$ Insulin resistance is present in the majority of people with the metabolic syndrome and is strongly associated with the risk of type 2 diabetes and cerebro- and cardiovascular diseases. It creates a physiologic state that significantly increases the likelihood of developing the metabolic abnormalities that comprise the metabolic syndrome, like glucose intolerance and dyslipidemia. Insulin resistance is also closely related to hypertension. Although this correlation is not completely understood, presumably enhanced renal sodium retention and increased sympathic nervous system activity play a role.

Epidemiological studies have shown that in individuals with increased body mass index (BMI), the prevalence and incidence of type 2 diabetes, dyslipidemia and cerebro- and cardiovascular disease subsequently increases. It also has been shown that among equally overweight or obese individuals, those that have an increase in abdominal fat (as assessed by waist circumference) are at increased risk of type 2 diabetes and cerebro- and cardiovascular disease. ${ }^{19,20}$

There is a strong and independent relationship between intra-abdominal fat and the metabolic syndrome. ${ }^{21}$ In the past several years, the view of adipose tissue has evolved considerably. Adipose tissue is now considered to be an active endocrine organ and appears to be a source of pro-inflammatory factors and of factors that play a role in the development of atherosclerosis. Several of these factors, including adipokines, free fatty acids and inflammatory mediators like tumor necrosis factor-alpha (TNF- $\alpha$ ) and interleukin (IL)-6, have been causally implicated in the development of insulin resistance. In obese and in insulin resistant subjects, excess amounts of free fatty acids and many other adipokines are released into the circulation. Increased free fatty acids levels can impair insulin sensitivity, promote hypercholesterolemia, and impact endothelial dysfunction, thereby promoting hypertension. Furthermore, adipokines enhance the cardiovascular risk as they play a role in dyslipidemia, hypertension and impaired glucose tolerance. This strengthens the association between obesity and inflammation. ${ }^{22}$ As abdominal obesity is one of the main components of the metabolic syndrome, the increasing prevalence of obesity worldwide is likely to be one of the main drivers of the increasing prevalence of the metabolic syndrome. 


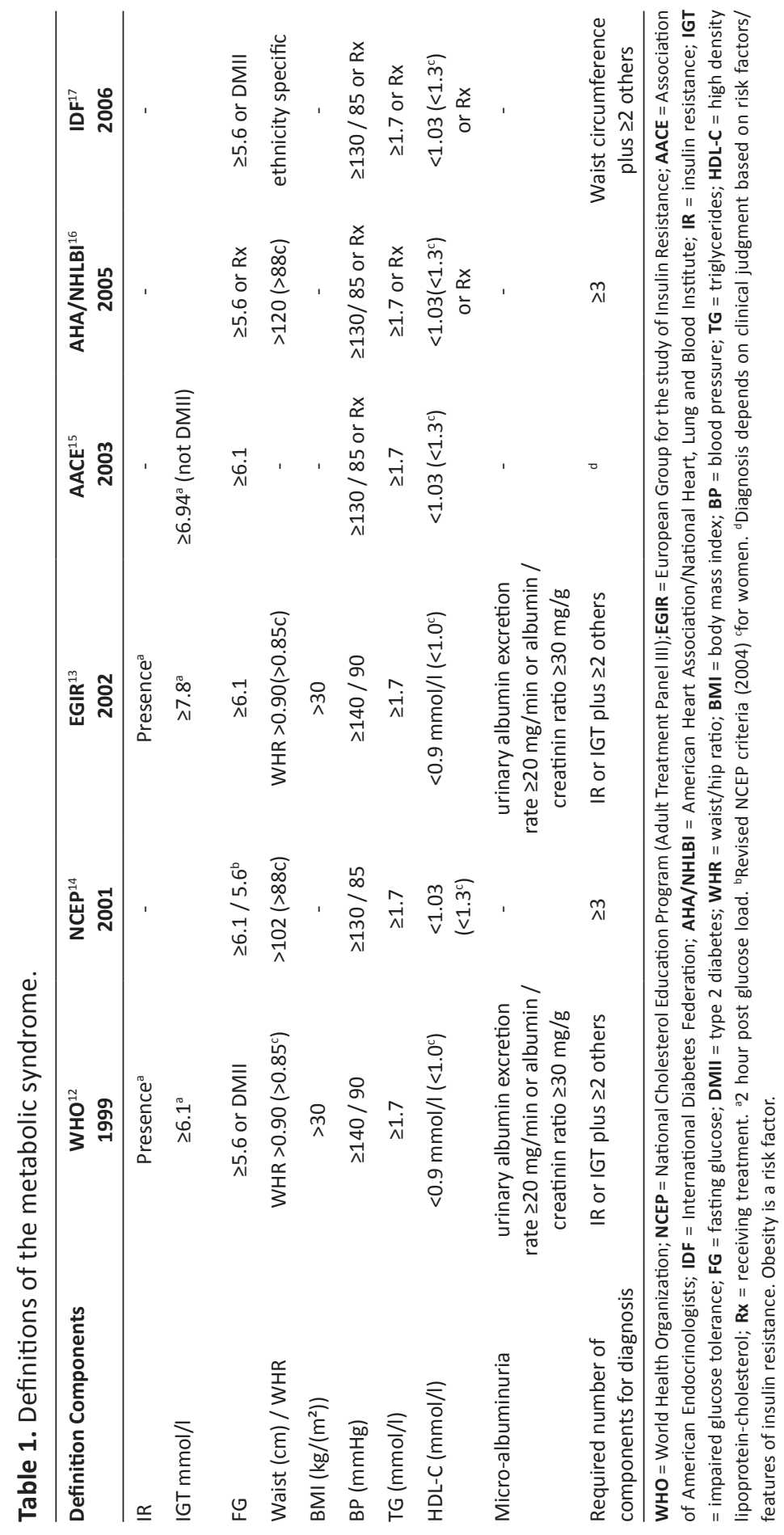




\section{Clinical relevance of the metabolic syndrome for the normal population Increased risk for cardiovascular disease}

As the metabolic syndrome includes several accepted cardiovascular and cerebrovascular risk factors, it is expected to be a strong predictor of cerebro- and cardiovascular disease. Several studies observed an increased risk for cardiovascular morbidity and mortality in individuals who met the criteria for the metabolic syndrome. ${ }^{23-25}$ Nevertheless, other studies doubt whether the metabolic syndrome predicts the cardiovascular risk better than the individual known cardiovascular risk factors. For example, McNeill et al. ${ }^{26}$ did not detect excess cardiovascular risk with the presence of the metabolic syndrome. However, in people that met the criteria for metabolic syndrome and fell in the category $<20 \%$ coronary heart disease risk, according to the Framingham Risk Score, a higher absolute rate of coronary heart disease was detected than in individuals without the metabolic syndrome. Golden et al. ${ }^{27}$ observed that several combinations of risk factors associated with the metabolic syndrome provided additional risk beyond the risk predicted by the individual components. Another study described the predictive value of the individual criteria and found that the risk for cardiovascular diseases increased when the number of components of the metabolic syndrome increased. ${ }^{25} \mathrm{It}$ is possible, that this inconsistency is related to differences in definition of the metabolic syndrome, alternatively this may be due to the fact that groups with different characteristics are used in these studies.

WHO and NCEP definitions have been compared concerning their predictive values for cardiovascular disease and type 2 diabetes. In these studies, a variety of estimated risks is reported. Although no consensus is reached as yet, it appears that the NCEP and the WHO definition can both predict cardiovascular diseases (Table 2).

Table 2. Comparison of metabolic syndrome definitions.

\begin{tabular}{lccccc}
\hline Reference & Population & CVD & DMII & Definitions & Preferred definition \\
\hline Lakka $^{23}$ & 1209 & $X$ & & WHO* | NCEP* & WHO \\
Hunt $^{24}$ & 2815 & $X$ & & WHO* | NCEP & NCEP \\
Laaksonen $^{28}$ & 1038 & & $X$ & WHO* | NCEP* & WHO \\
Lorenzo $^{75}$ & 1734 & & $X$ & WHO* | NCEP & NCEP \\
\hline
\end{tabular}

CVD = Cardiovascular diseases; DMII = Type 2 diabetes mellitus; $\mathbf{W H O}=$ World Health Organization; NCEP = National Cholesterol Education Program/Adult Treatment Panel III. *Modified definition.

\section{Increased risk for type 2 diabetes}

As type 2 diabetes, insulin resistance, impaired glucose tolerance and increased fasting plasma glucose are components of the metabolic syndrome, it is difficult to predict the risk of type 2 diabetes in subjects with the metabolic syndrome. Several studies suggest that subjects with the metabolic syndrome, have an increased risk of developing type 2 diabetes. ${ }^{28,29} \mathrm{WHO}$ and 
NCEP definitions have been compared concerning their predictive values for diabetes (Table 2). Both definitions identified persons at high risk for developing type 2 diabetes. Thus far, there is no consensus about the most appropriate definition for the metabolic syndrome. In general the NCEP definition and WHO definition both provide similar predictive values for cardiovascular diseases and type 2 diabetes, however the NCEP definition holds the best clinical usefulness.

\section{Methods}

A review of the available literature on the metabolic syndrome in adult survivors of childhood cancer, but also on obesity, dyslipidemia, hypertension and insulin resistance, i.e. the components of the metabolic syndrome, was performed using PubMed data bases (National Library of Medicine, Washington, DC) until June 2008. The keywords 'survivors of childhood cancer', 'metabolic syndrome', 'insulin resistance', 'obesity', 'dyslipidemia' and 'hypertension' were used. Inclusion criterium for analysis was that the study reported survivors aged 18 years or older.

\section{Results}

\section{Metabolic syndrome in adult survivors of childhood cancer}

To date several studies have focused on the possible relationship between treatment of childhood cancer and an increased risk of developing risk factors contributing to the metabolic syndrome. However, these studies have limited impact because of small sample sizes and lack of optimal matched groups. Moreover, in other studies only components of the metabolic syndrome, like obesity, have been studied. To our knowledge, so far only four studies described the metabolic syndrome in adult long-term survivors of childhood cancer (Table 3). Trimis et al. assessed the metabolic syndrome according to a modified definition in 80 survivors of acute lymphoblastic leukemia (ALL) (median age at follow-up 13.9 years, range 5.2 - 24.1; median follow-up time 6.3 years, range 1.1 - 12.2). They found a 2 -fold increased prevalence of the metabolic syndrome in patients treated with chemotherapy only and a 5 -fold increased prevalence in patients treated with chemotherapy and radiotherapy, compared with the normal population. ${ }^{30}$ Another study assessed the metabolic syndrome in 75 childhood ALL survivors (mean age at follow-up 30 years, mean follow-up time 25 years) and found a prevalence of $16.6 \%$. Although this prevalence was not significantly different from population norms, it was striking that $60 \%$ of survivors treated with cranial irradiation, compared to $20 \%$ of those who were not, had at least two components of the metabolic syndrome. A strong relation between cranial irradiation and growth hormone $(\mathrm{GH})$ deficiency, and in turn components of the metabolic syndrome, was found. ${ }^{5}$ Damage to the hypothalamic-pituitary 


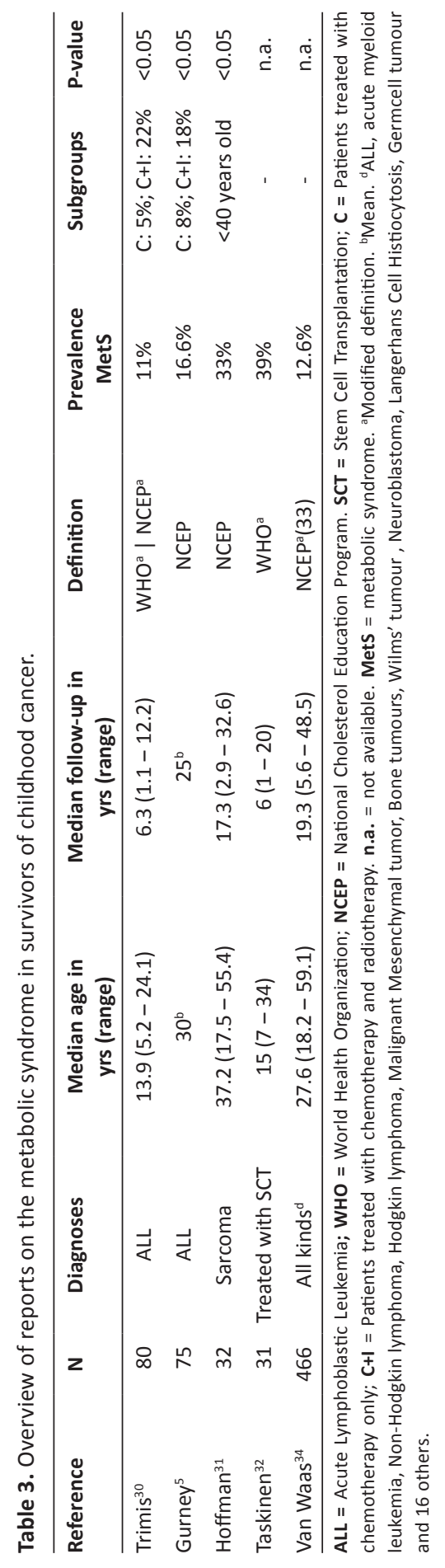


axis from cranial irradiation is largely responsible for the long-term effects of GH deficiency. $\mathrm{GH}$ deficiency is strongly associated with abnormal vascular reactivity and development of atherosclerosis, and also with a pattern of dyslipidemia and abdominal obesity. Hoffman et al. ${ }^{31}$ determined the metabolic syndrome in sarcoma survivors (median age at follow-up 37.2 years, range 17.5 - 55.4 years; follow-up time 17.3 years, range $2.9-32.6$ years) and found an increased prevalence among subjects aged $20-39$ years. In male survivors, testosterone levels declined as the number of metabolic syndrome traits increased, indicating that gonadal function and metabolic syndrome might influence each other. Moreover, GH deficiency was seen both in those who received cranial irradiation and in those who did not. Another potential mechanism for the development of the metabolic syndrome in at least a subgroup of sarcoma survivors is physical inactivity, as this promotes obesity and subsequent insulin resistance. Taskinen et al. ${ }^{32}$ described long-term survivors (median age at follow-up 15 years, range 7 - 34 years; median follow-up time 6, range 1 - 20 years) who were transplanted for leukemia or nonmalignant hematological diseases and observed 12 out of 31 to have the metabolic syndrome. GH deficiency clustered among the patients with metabolic syndrome, whereas metabolic syndrome was found in $60 \%$ of the patients with GH deficiency, in contrast to only $19 \%$ of the patients with normal GH levels. Except for GH deficiency, the patients with or without the metabolic syndrome did not differ in abnormalities in hormones such as thyroid hormones, sex hormones and androgens.

We assessed the metabolic syndrome according to a modified NCEP definition, that was used previously by Haugnes et al., ${ }^{33}$ in a heterogeneous group of 466 adult childhood cancer survivors. We found a prevalence of $12.6 \%$ in this relatively young group (median age at follow-up 27.6 years; range $18.2-59.1) .^{34}$

\section{Factors contributing to the metabolic syndrome in adult survivors of childhood cancer} Many studies do not report on the metabolic syndrome but on various components of the metabolic syndrome in adult survivors of childhood cancer.

\section{Insulin resistance / Diabetes mellitus}

Insulin resistance, hyperinsulinemia and impaired glucose tolerance have been assessed in survivors of several childhood malignancies (Table 4).9,10,35-38 Patients treated with stem cell transplantation and total body irradiation seem to be at an increased risk of developing one of these states. Neville et al. ${ }^{9}$ found that stem cell transplantation with total body irradiation is a prominent risk factor for the development of hyperinsulinemia, impaired glucose tolerance, or type 2 diabetes in a heterogeneous group of 248 survivors. Especially pubertal and adult subjects seem to be at risk. However, the risk was not limited to subjects who had undergone stem cell transplantation, and untreated hypogonadism and abdominal adiposity also emerged as important. Taskinen et al. ${ }^{10}$ also found an association between stem cell transplantation and hyperinsulinemia and impaired glucose tolerance. Another study found hyperinsulinemia in three out of 17 survivors after stem cell transplantation. ${ }^{36}$ 


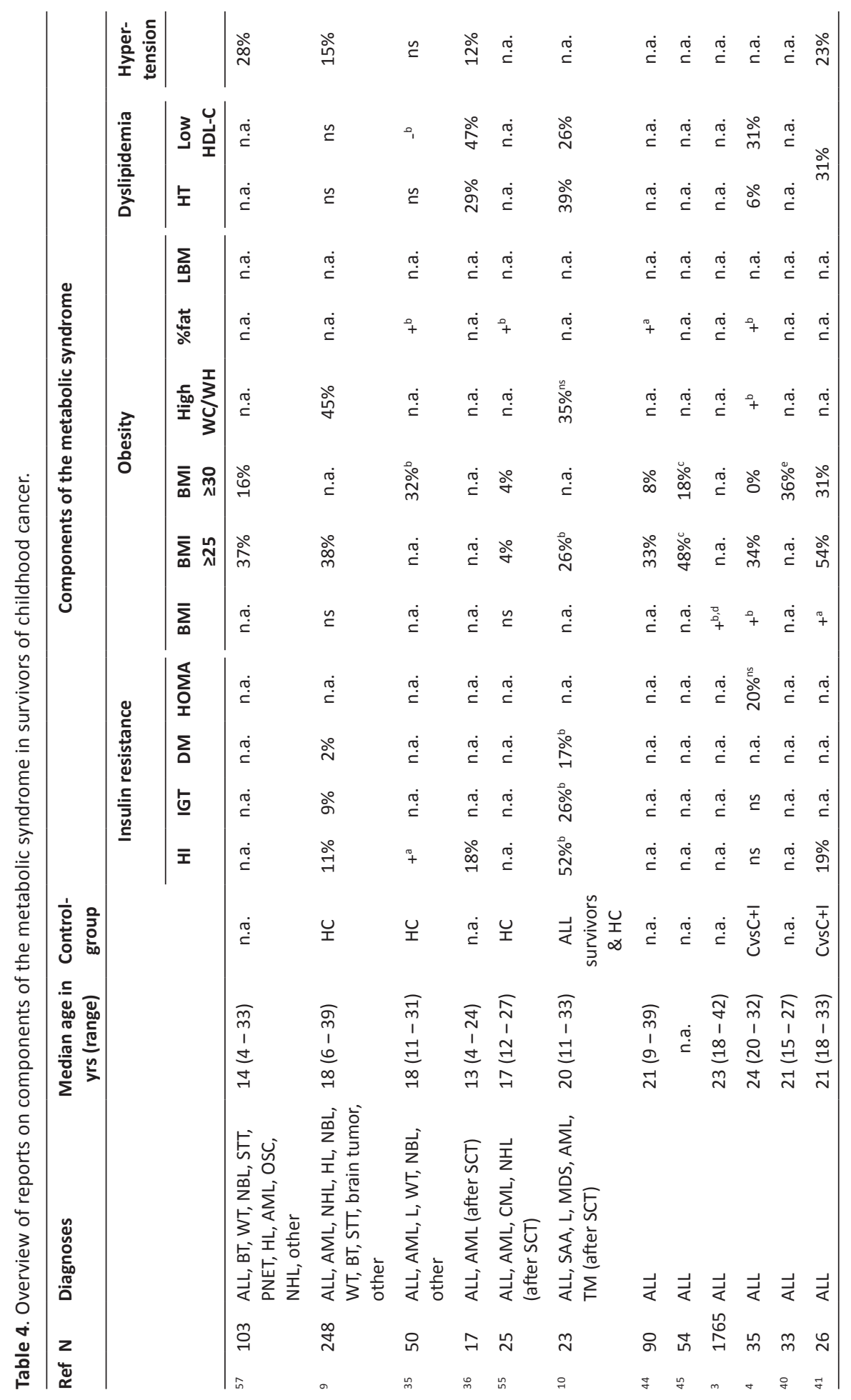




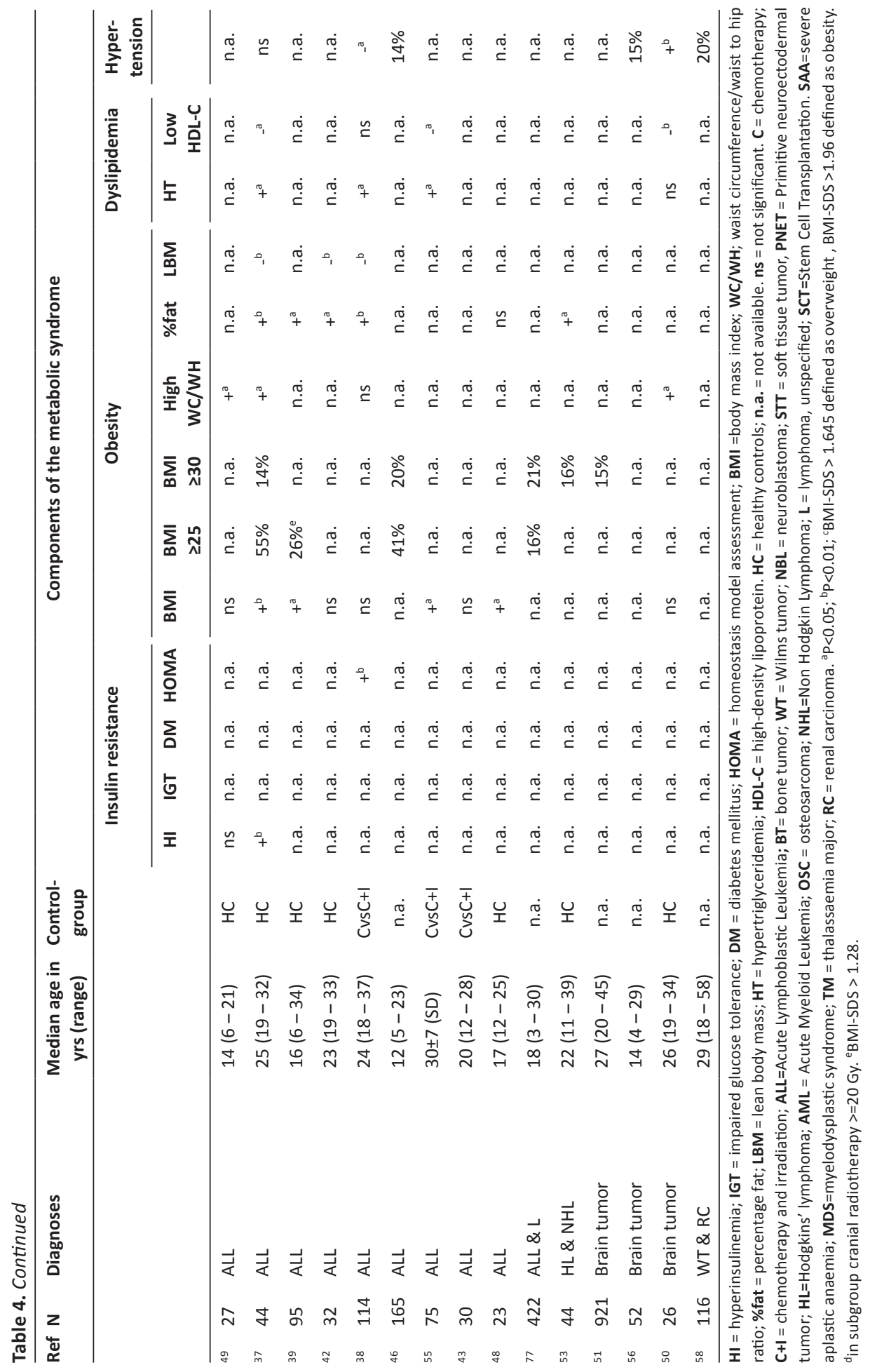


Survivors treated with cranial irradiation also seem to be at increased risk of developing insulin resistance, hyperinsulinemia and impaired glucose tolerance. Survivors of ALL treated with chemotherapy and cranial irradiation tend to have increased plasma levels of insulin and blood glucose compared to healthy controls. ${ }^{37}$ Another study found increased homeostasis model assessment of insulin resistance (HOMA) scores and fasting glucose levels in cranially irradiated survivors of ALL compared with survivors who did not receive cranial irradiation. ${ }^{38}$ Talvensaari et al. found significantly higher plasma fasting glucose and insulin concentrations both in survivors of leukemia and lymphoma treated with cranial irradiation, testicular irradiation and chemotherapy, and in survivors of solid tumors treated with surgery, local irradiation and chemotherapy. Yet, the latter group consisted only of four patients. Still, they also found a triad of hyperinsulinemia, obesity and low HDL-C concentration in eight out of 50 patients, of whom four did not receive cranial irradiation, indicating that cranial irradiation might not be the only contributing factor. ${ }^{35}$ Normal serum insulin concentrations, fasting plasma glucose and HOMA-indexes have also been assessed in groups of survivors of ALL treated with and without cranial irradiation. ${ }^{4}$

\section{Obesity}

A well-described phenomenon in ALL patients who were treated with cranial irradiation is obesity (Table 4). This can be explained by GH deficiency, resulting from damage to the hypothalamicpituitary axis, which occurs more frequently in irradiated patients. ${ }^{35}$ However, GH deficiency is probably not the only cause. Many studies found increased prevalence of obesity in cranially irradiated patients, ${ }^{3,4,37-43}$ but others described this increased prevalence also in patients who received chemotherapy only. ${ }^{40,44-46} \mathrm{~A}$ study from our institute showed that patients treated with and without cranial irradiation did not differ in weight gain four years after cessation of therapy. Children treated with chemotherapy including steroids, however, did have an increased $\mathrm{BMI}$, indicating that not cranial irradiation but chemotherapy including steroids explains the weight gain in children treated for ALL. ${ }^{47}$ We also observed an increased BMI in ALL survivors. Additionally, cranially irradiated patients had an increased percentage of body fat and decreased lean body mass and bone mineral content, compared to non-irradiated patients. Patients treated with prednisolone also had an increased percentage of body fat. Percentage fat was normal in dexamethasone treated patients, however, there was no significant difference between prednisolone treated and dexamethasone treated patients. ${ }^{44}$ Another study described a higher percent fat in survivors of ALL, significantly related to cranial irradiation or GH deficiency, but not to cumulative doses of steroids. ${ }^{39}$ Van der Sluis et al. ${ }^{48}$ studied ALL survivors treated with dexamethasone and methothrexate and did not find an elevated percentage fat or reduced lean body mass.

Several studies found increased levels of leptin in ALL survivors treated with cranial irradiation. ${ }^{37,38,42,49}$ Birkebaek et al. found serum levels of leptin, independently from GH status, to be increased in irradiated survivors compared to non-irradiated survivors. ${ }^{43}$ Leptin, a hormone produced by adipose tissue, acts as an afferent signal of satiation in a feedback loop involving 
areas of the brain associated with appetite and satiety. The final effect of this loop is the regulation of body fat. Obese subjects have high plasma leptin levels, suggestive for leptin resistance. Therefore, it is possible that high serum leptin levels in these survivors are a direct consequence of radiation-induced hypothalamic damage or an indirect effect produced by centrally induced GH deficiency.

Besides in ALL survivors, obesity has also been described in survivors of other types of childhood cancer, ${ }^{9,35}$ including (subgroups of) survivors of brain tumors. ${ }^{50,51}$ Also, we estimated the body composition in long-term survivors of Hodgkin's disease, who were treated without radiotherapy, and found an increased percentage body fat in male and female patients treated with chemotherapy containing prednisolone. ${ }^{52}$ Nysom et al. did similar findings in non Hodgkin Lymphoma and Hodgkin's disease survivors ${ }^{53}$ and in a group of survivors of leukemia and lymphoma who were treated with stem cell transplantation. ${ }^{54}$ These findings indicate that, besides survivors of ALL, survivors of other types of childhood cancer might also be at risk of developing obesity.

\section{Dyslipidemia}

Dyslipidemia has been described in survivors of ALL, brain tumors and after stem cell transplantation (Table 4). Decreased levels of $\mathrm{HDL}-\mathrm{C}^{4,10,35-38,50}$ and increased levels of triglycerides ${ }^{4,10,32,36-38,41,55}$ and low-density lipoprotein-cholesterol (LDL-C) levels, ${ }^{4,36-38,50,55}$ are the most described forms of dyslipidemia in survivors of childhood cancer. Other manifestations of dyslipidemia that have been described in survivors of childhood cancer are increased total cholesterol, ${ }^{36,37,55}$ very low-density lipoprotein-cholesterol (VLDL-C) ${ }^{41}$ and apolipoprotein (apo)-B levels, ${ }^{36,49}$ and decreased apo-A1 levels. ${ }^{36,49}$ All these studies suggested that GH deficiency, presumably caused by hypothalamic damage after cranial irradiation, or total body irradiation as part of the conditioning regimen for stem cell transplantation, is involved in the pathogenesis of dyslipidemia. This indicates that GH may play an important role in the regulation of lipoprotein metabolism during adult life. However, Dengel et al. found that ALL survivors treated with chemotherapy only had significantly higher triglyceride levels compared with healthy controls. On the other hand, survivors who were also treated with cranial irradiation had a significantly increased total cholesterol, LDL-C, triglyceride levels and decreased HDL-C as compared to both a healthy control group and a chemotherapy-only group..$^{55} \mathrm{~A}$ study with $26 \mathrm{ALL}$ survivors described a difference between survivors treated with cranial irradiation and those who where not, with respect to triglyceride and VLDL-C levels, but not to total cholesterol, LDL-C and HDL-C. ${ }^{41}$

Endothelial function was not different between a control group and ALL survivors, treated with chemotherapy only and treated with chemotherapy and cranial irradiation after a mean follow-up of 24.6 years (SD \pm 4.8 ). ${ }^{55}$ Increased intima media thickness in the carotic bulb ${ }^{50}$ and renal glomerular dysfunction and changes in renal tubular functions ${ }^{55}$ have been described in survivors of brain tumor, presumably due to GH deficiency and cisplatin treatment, respectively (Table 4). 


\section{Hypertension}

As obesity has been described very often in survivors of childhood cancer, it is conceivable that hypertension may occur often as well. Blood pressure has been investigated in survivors of several malignancies and outcomes have varied (Table 4). Haddy et al. ${ }^{57}$ found (pre-) hypertension in $28.2 \%$ of childhood cancer survivors. Seven out of 10 Wilms' tumor survivors were found to have (pre-) hypertension, however, comparison with a normal control group was not available. Increased prevalence of hypertension has been assessed in survivors of ALL, but the studies were limited by lack of control group or loss to follow-up, resulting in a small amount of patients. ${ }^{41,45}$ Interestingly, Kantor et al. ${ }^{58}$ did not find a significant difference between blood pressure in survivors of renal cancers and the normal population. Survivors of brain tumors and survivors after hematopoietic stem transplant have also been described to suffer from hypertension. ${ }^{50,56}$ In heterogeneous group of our 440 adult survivors aged $20-40$ years, we found hypertension in $24 \%$, which was significantly higher compared to the normal Dutch population. ${ }^{34}$

\section{Discussion}

Based upon studies of (components of) the metabolic syndrome in childhood cancer survivors, it seems to be likely that adult survivors of childhood cancer are at increased risk. Especially the prevalence of obesity seems to be increased in adult survivors of childhood cancer, however, increased prevalence of insulin resistance, dyslipidemia and hypertension have also been reported.

A possible explanation for this increased risk might be the presence of GH deficiency. Several studies reported GH deficiency in survivors, particularly in patients who received cranial irradiation, $5,30,31,35,37,42,43,50$ but also in patients who were treated with chemotherapy only, $5,31,35,43$ or with stem cell transplantation. ${ }^{32,37}$ Described prevalences of GH deficiency in adult survivors of childhood cancer vary between $35 \%$ and $91 \% .{ }^{5,9,31,32,37,38,44,51}$ It is known that children ${ }^{60}$ and adults ${ }^{60}$ who are $\mathrm{GH}$ deficient for other reasons are more often obese. Moreover, increased total cholesterol, LDL-C, apo-B levels, triglycerides and lipoprotein(a) levels, and a normal or decreased HDL-C, were found in GH deficient patients. ${ }^{61-63}$ Also, increased peripheral insulin resistance and impaired glucose tolerance were observed in these subjects. ${ }^{64}$ Accordingly, GH deficiency might be involved in the development of the components of the metabolic syndrome.

GH deficiency may be caused by direct pituitary damage resulting from cranial irradiation, however, it is also possible that it is caused by dysregulation of hypothalamic hormones, following from hypothalamic damage. The hypothalamus is more sensitive than the pituitary to the effects of radiation. Hypothalamic damage may also be a potential cause of insensitivity for leptin, a hormone involved in the regulation of appetite and metabolism. Subsequently, insensitivity for leptin can cause obesity it self. 
GH deficiency has also been determined in survivors who had been treated with chemotherapy only. This might be explained by chemotherapy crossing the blood-brain barrier in combination with individual susceptibility. ${ }^{65}$ Other endocrinopathies that might be associated with an increased risk of developing components of the metabolic syndrome are thyroid dysfunction and gonadal dysfunction. Thyroid dysfunction, particularly primary hypothyroidism, has been described after radiotherapy and after chemotherapy, especially cytokines. In survivors of pediatric Hodgkin's disease treated with neck irradiation, the prevalence ranged from $30-50 \%$, depending on the dosage. Approximately $8 \%$ of the patients who were treated with chemotherapy only also had been diagnosed with hypothyroidism. ${ }^{65}$ It is known that hypothyroidism causes modest weight gain, which is largely the result of fluid retention and decreased metabolism. Decreased thyroid hormone effect can cause increased levels of total cholesterol and LDL-C and a possible change in HDL-C due to a change in metabolic clearance. In addition, hypothyroidism may result in an increase in insulin resistance. Moreover, hypothyroidism causes fatigue and loss of energy, possibly contributing to the increase in weight.

Gonadal dysfunction has been described mainly after radiotherapy, alkylating agents and gonadotoxic drugs. ${ }^{67} \mathrm{~A}$ direct explanation for this is not yet available, but it is hypothesized that hormonal alterations induce obesity, which subsequently induces insulin resistance and other components of the metabolic syndrome. Alternatively, hormonal alterations might be induced by obesity.

The metabolic syndrome increases the risk of cardiovascular and cerebrovascular diseases. Some survivors are already at increased risk of developing these diseases. For example, stroke has been reported after Hodgkin's disease ${ }^{68}$ and after ALL and brain tumors. ${ }^{69} \mathrm{~A}$ possible explanation for this increased risk is carotid artery disease following from radiotherapy. This is believed to be a result of premature atherosclerotic changes in the arterial wall, including endothelial cell damage. Another possible cause for the increased risk of developing components of the metabolic syndrome might be therapy including corticosteroids. It is known that children who receive corticosteroids gain weight. However, it remains unclear why this increase continues long after treatment is stopped. It is suggested that the development of sequential endocrinopathies might be the explanation.

Other factors that are suggested to be involved in the development of the metabolic syndrome are reduced physical activity or a sedentary lifestyle. Because survivors of childhood cancer have been described to suffer from many chronic health conditions, ${ }^{70}$ for example cardiac dysfunction due to anthracyclines or renal failure due to radiotherapy or cisplatin, a reduced physical activity is to be expected. Several years after cessation of therapy, the resting energy expenditure of survivors is similar to that in controls, ${ }^{71}$ or slightly lower. ${ }^{72}$ This reduced physical activity, together with altered energy equilibrium, can contribute to obesity.

There are a few limitations to this review; the reviewed studies are heterogeneous with respect to diagnosis, treatment, age and follow-up time, but also with respect to used definitions and cut-off values for the (components of) the metabolic syndrome. In addition, methodological 
quality of the described studies was often low and as described above no clear pathophysiological explanation is given. However, this taken into account, based upon the studies (subgroups of) survivors of childhood cancer seem to be at increased risk of developing one or more components of the metabolic syndrome.

Although treatment regimens that have been used in adult survivors are not the same as the regimens that are used nowadays, knowledge of the possible late effects of these regimens is very important. Determining the components of the metabolic syndrome in the follow-up of childhood cancer survivors is of great importance as the number of adult survivors is increasing. As some of the components of the metabolic syndrome, like dyslipidemia and hypertension, are often asymptomatic, screening plays an important role in this follow-up.

Up till now it remains unclear whether the metabolic syndrome should and can be treated by a single therapy targeting all or multiple risk factors. ${ }^{72}$ Until such a treatment is available, single risk factors should be treated separately. Furthermore, GH deficiency and other endocrinopathies should be investigated and treated. GH treatment has been well proved to improve lipid metabolism and body composition and to enhance quality of life. ${ }^{74}$ Dyslipidemia and hypertension also require medical management. Type 2 diabetes should be treated as soon as possible with conventional therapy. The use of insulin sensitizers and biguanides, like metformin and thiazolidinediones, in the treatment of insulin resistance has been proposed, however no consensus is reached as yet. ${ }^{75}$

Additionally, treatment of the metabolic syndrome should include losing weight by increased physical activity and an appropriate diet and may require a medical intervention. This all because (early) treatment of the components of the metabolic syndrome may prevent cardiovascular events. To assure the actual role of medical treatment and lifestyle changes, intervention studies should be conducted.

In conclusion, long-term survivors of childhood cancer seem to be at increased risk of developing (components of) the metabolic syndrome and should be screened for these components. To determine the best intervention for these survivors, future intervention studies are necessary. 


\section{References}

1. Meadows AT. Pediatric cancer survivors: Past history and future challenges. Curr Probl Cancer 2003;27:112-26.

2. Robison LL, Green DM, Hudson M, et al. Long-term outcomes of adult survivors of childhood cancer. Cancer 2005;104:2557-64.

3. Oeffinger KC, Mertens AC, Sklar CA, et al. Obesity in adult survivors of childhood acute lymphoblastic leukemia: a report from the Childhood Cancer Survivor Study. J Clin Oncol 2003;21:1359-65.

4. Jarfelt M, Lannering B, Bosaeus I, Johannsson G, Bjarnason R. Body composition in young adult survivors of childhood acute lymphoblastic leukemia. Eur J Endocrinol 2005;153:81-9.

5. Gurney JG, Ness KK, Sibley SD, et al. Metabolic syndrome and growth hormone deficiency in adult survivors of childhood acute lymphoblastic leukemia. Cancer 2006;107:1303-12.

6. van Casteren NJ, van der Linden GH, Hakvoort-Cammel FG, Hahlen K, Dohle GR, van den Heuvel-Eibrink MM. Effect of childhood cancer treatment on fertility markers in adult male long-term survivors. Pediatr Blood Cancer 2009;52:108-12.

7. Lie Fong S, Laven JS, Hakvoort-Cammel FG, et al. Assessment of ovarian reserve in adult childhood cancer survivors using anti-Mullerian hormone. Human Reprod (Oxford, England) 2009;24:982-90.

8. MacArthur AC, Spinelli JJ, Rogers PC, Goddard KJ, Abanto ZU, McBride ML. Mortality among 5-year survivors of cancer diagnosed during childhood or adolescence in British Columbia, Canada. Pediatr Blood Cancer 2007;48:460-7.

9. Neville KA, Cohn RJ, Steinbeck KS, Johnston K, Walker JL. Hyperinsulinemia, impaired glucose tolerance, and diabetes mellitus in survivors of childhood cancer: prevalence and risk factors. J Clin Endocrinol Metab 2006;91:4401-7.

10. Taskinen M, Saarinen-Pihkala UM, Hovi L, Lipsanen-Nyman M. Impaired glucose tolerance and dyslipidemia as late effects after bone-marrow transplantation in childhood. Lancet 2000;356:993-7.

11. Reaven GM. Banting lecture 1988. Role of insulin resistance in human disease. Diabetes 1988;37:1595607.

12. Alberti KG, Zimmet PZ. Definition, diagnosis and classification of diabetes mellitus and its complications. Part 1: diagnosis and classification of diabetes mellitus provisional report of a WHO consultation. Diabet Med 1998;15:539-53.

13. Balkau B, Charles MA, Drivsholm T, et al. Frequency of the WHO metabolic syndrome in European cohorts, and an alternative definition of an insulin resistance syndrome. Diabetes Metab 2002;28:36476.

14. Executive Summary of The Third Report of The National Cholesterol Education Program (NCEP) Expert Panel on Detection, Evaluation, And Treatment of High Blood Cholesterol In Adults (Adult Treatment Panel III). JAMA 2001;285:2486-97.

15. Einhorn D, Reaven GM, Cobin RH, et al. American College of Endocrinology position statement on the insulin resistance syndrome. Endocr Pract 2003;9:237-52.

16. Grundy SM, Cleeman JI, Daniels SR, et al. Diagnosis and management of the metabolic syndrome: an American Heart Association/National Heart, Lung, and Blood Institute Scientific Statement. Circulation 2005;112:2735-52.

17. Alberti KG, Zimmet P, Shaw J. Metabolic syndrome - a new world-wide definition. A Consensus Statement from the International Diabetes Federation. Diabet Med 2006;23:469-80.

18. Grundy SM. Does the metabolic syndrome exist? Diabetes Care 2006;29:1689-92; discussion 93-6.

19. Rexrode KM, Carey VJ, Hennekens $\mathrm{CH}$, et al. Abdominal adiposity and coronary heart disease in women. JAMA 1998;280:1843-8.

20. Ohlson LO, Larsson B, Svardsudd K, et al. The influence of body fat distribution on the incidence of diabetes mellitus. 13.5 years of follow-up of the participants in the study of men born in 1913. Diabetes 1985;34:1055-8. 
21. Carr DB, Utzschneider KM, Hull RL, et al. Intra-abdominal fat is a major determinant of the National Cholesterol Education Program Adult Treatment Panel III criteria for the metabolic syndrome. Diabetes 2004;53:2087-94.

22. Fonseca VA. The metabolic syndrome, hyperlipidemia, and insulin resistance. Clinical Cornerstone 2005;7:61-72.

23. Lakka HM, Laaksonen DE, Lakka TA, et al. The metabolic syndrome and total and cardiovascular disease mortality in middle-aged men. JAMA 2002;288:2709-16.

24. Hunt KJ, Resendez RG, Williams K, Haffner SM, Stern MP. National Cholesterol Education Program versus World Health Organization metabolic syndrome in relation to all-cause and cardiovascular mortality in the San Antonio Heart Study. Circulation 2004;110:1251-7.

25. Malik S, Wong ND, Franklin SS, et al. Impact of the metabolic syndrome on mortality from coronary heart disease, cardiovascular disease, and all causes in United States adults. Circulation 2004;110:1245-50.

26. McNeill AM, Katz R, Girman CJ, et al. Metabolic syndrome and cardiovascular disease in older people: The cardiovascular health study. J Am Geriatr Soc 2006;54:1317-24.

27. Golden SH, Folsom AR, Coresh J, Sharrett AR, Szklo M, Brancati F. Risk factor groupings related to insulin resistance and their synergistic effects on subclinical atherosclerosis: the atherosclerosis risk in communities study. Diabetes 2002;51:3069-76.

28. Laaksonen DE, Lakka HM, Niskanen LK, Kaplan GA, Salonen JT, Lakka TA. Metabolic syndrome and development of diabetes mellitus: application and validation of recently suggested definitions of the metabolic syndrome in a prospective cohort study. Am J Epidemiol 2002;156:1070-7.

29. Lorenzo C, Williams K, Hunt KJ, Haffner SM. The National Cholesterol Education Program - Adult Treatment Panel III, International Diabetes Federation, and World Health Organization definitions of the metabolic syndrome as predictors of incident cardiovascular disease and diabetes. Diabetes Care 2007;30:8-13.

30. Trimis G, Moschovi M, Papassotiriou I, Chrousos G, Tzortzatou-Stathopoulou F. Early indicators of dysmetabolic syndrome in young survivors of acute lymphoblastic leukemia in childhood as a target for preventing disease. J Pediatr Hematol Oncol 2007;29:309-14.

31. Hoffman KE, Derdak J, Bernstein D, et al. Metabolic syndrome traits in long-term survivors of pediatric sarcoma. Pediatr Blood Cancer 2008;50:341-6.

32. Taskinen M, Lipsanen-Nyman M, Tiitinen A, Hovi L, Saarinen-Pihkala UM. Insufficient growth hormone secretion is associated with metabolic syndrome after allogeneic stem cell transplantation in childhood. J Pediatr Hematol Oncol 2007;29:529-34.

33. Haugnes HS, Aass N, Fossa SD, et al. Components of the metabolic syndrome in long-term survivors of testicular cancer. Ann Oncol 2007;18:241-8.

34. van Waas M, Neggers SJ, Pieters R, van den Heuvel-Eibrink MM. Components of the metabolic syndrome in 500 adult long-term survivors of childhood cancer. Ann Oncol 2010;21:1121-6.

35. Talvensaari KK, Lanning M, Tapanainen P, Knip M. Long-term survivors of childhood cancer have an increased risk of manifesting the metabolic syndrome. J Clin Endocrinol Metab 1996;81:3051-5.

36. Perkins JL, Kunin-Batson AS, Youngren NM, et al. Long-term follow-up of children who underwent hematopoeitic cell transplant (HCT) for AML or ALL at less than 3 years of age. Pediatr Blood Cancer 2007;49:958-63.

37. Link K, Moell C, Garwicz S, et al. Growth hormone deficiency predicts cardiovascular risk in young adults treated for acute lymphoblastic leukemia in childhood. J Clin Endocrinol Metab 2004;89:500312.

38. Janiszewski PM, Oeffinger KC, Church TS, et al. Abdominal obesity, liver fat, and muscle composition in survivors of childhood acute lymphoblastic leukemia. J Clin Endocrinol Metab 2007;92:3816-21.

39. Nysom K, Holm K, Michaelsen KF, Hertz H, Muller J, Molgaard C. Degree of fatness after treatment for acute lymphoblastic leukemia in childhood. J Clin Endocrinol Metab 1999;84:4591-6.

40. Birkebaek NH, Clausen N. Height and weight pattern up to 20 years after treatment for acute lymphoblastic leukemia. Arch Dis Child 1998;79:161-4. 
41. Oeffinger KC, Buchanan GR, Eshelman DA, et al. Cardiovascular risk factors in young adult survivors of childhood acute lymphoblastic leukemia. J Pediatr Hematol Oncol 2001;23:424-30.

42. Brennan BM, Rahim A, Blum WF, Adams JA, Eden OB, Shalet SM. Hyperleptinemia in young adults following cranial irradiation in childhood: growth hormone deficiency or leptin insensitivity? Clin Endocrinol 1999;50:163-9.

43. Birkebaek NH, Fisker S, Clausen N, Tuovinen V, Sindet-Pedersen S, Christiansen JS. Growth and endocrinological disorders up to 21 years after treatment for acute lymphoblastic leukemia in childhood. Med Pediatr Oncol 1998;30:351-6.

44. van Beek RD, de Muinck Keizer-Schrama SM, Hakvoort-Cammel FG, et al. No difference between prednisolone and dexamethasone treatment in bone mineral density and growth in long term survivors of childhood acute lymphoblastic leukemia. Pediatr Blood Cancerr 2006;46:88-93.

45. Chow EJ, Pihoker C, Hunt K, Wilkinson K, Friedman DL. Obesity and hypertension among children after treatment for acute lymphoblastic leukemia. Cancer 2007;110:2313-20.

46. Asner S, Ammann RA, Ozsahin H, Beck-Popovic M, von der Weid NX. Obesity in long-term survivors of childhood acute lymphoblastic leukemia. Pediatr Blood Cancer 2008;51:118-22.

47. Van Dongen-Melman JE, Hokken-Koelega AC, Hahlen K, De Groot A, Tromp CG, Egeler RM. Obesity after successful treatment of acute lymphoblastic leukemia in childhood. Pediatr Res 1995;38:86-90.

48. van der Sluis IM, van den Heuvel-Eibrink MM, Hahlen K, Krenning EP, de Muinck Keizer-Schrama SM. Bone mineral density, body composition, and height in long-term survivors of acute lymphoblastic leukemia in childhood. Med Pediatr Oncol 2000;35:415-20.

49. Papadia C, Naves LA, Costa SS, Vaz JA, Domingues L, Casulari LA. Incidence of obesity does not appear to be increased after treatment of acute lymphoblastic leukemia in Brazilian children: role of leptin, insulin, and IGF-1. Horm Res 2007;68:164-70.

50. Heikens J, Ubbink MC, van der Pal HP, et al. Long term survivors of childhood brain cancer have an increased risk for cardiovascular disease. Cancer 2000;88:2116-21.

51. Gurney JG, Ness KK, Stovall M, et al. Final height and body mass index among adult survivors of childhood brain cancer: childhood cancer survivor study. J Clin Endocrinol Metab 2003;88:4731-9.

52. van Beek RD, van den Heuvel-Eibrink MM, Hakvoort-Cammel FG, et al. Bone mineral density, growth and thyroid function in long-term survivors of pediatric Hodgkin's lymphoma treated with chemotherapy only. J Clin Endocrinol Metab 2009 Jun;94(6):1904-9.

53. Nysom K, Holm K, Michaelsen KF, Hertz H, Muller J, Molgaard C. Degree of fatness after treatment of malignant lymphoma in childhood. Med Pediatr Oncol 2003;40:239-43.

54. Nysom K, Holm K, Michaelsen KF, et al. Degree of fatness after allogeneic BMT for childhood leukemia or lymphoma. Bone Marrow Transplant 2001;27:817-20.

55. Dengel DR, Ness KK, Glasser SP, Williamson EB, Baker KS, Gurney JG. Endothelial function in young adult survivors of childhood acute lymphoblastic leukemia. J Pediatr Hematol Oncol 2008;30:20-5.

56. Pietila S, Ala-Houhala M, Lenko HL, Harmoinen AP, Turjanmaa V, Makipernaa A. Renal impairment and hypertension in brain tumor patients treated in childhood are mainly associated with cisplatin treatment. Pediatr Blood Cancer 2005;44:363-9.

57. Haddy TB, Mosher RB, Reaman GH. Hypertension and prehypertension in long-term survivors of childhood and adolescent cancer. Pediatr Blood Cancer 2007;49:79-83.

58. Kantor AF, Li FP, Janov AJ, Tarbell NJ, Sallan SE. Hypertension in long-term survivors of childhood renal cancers. J Clin Oncol 1989;7:912-5.

59. Boot AM, Engels MA, Boerma GJ, Krenning EP, De Muinck Keizer-Schrama SM. Changes in bone mineral density, body composition, and lipid metabolism during growth hormone (GH) treatment in children with GH deficiency. J Clin Endocrinol Metab 1997;82:2423-8.

60. Binnerts A, Deurenberg P, Swart GR, Wilson JH, Lamberts SW. Body composition in growth hormonedeficient adults. Am J Clin Nutr 1992;55:918-23.

61. de Boer H, Blok GJ, Voerman HJ, Phillips M, Schouten JA. Serum lipid levels in growth hormonedeficient men. Metabolism 1994;43:199-203. 
62. Angelin B, Olivecrona H, Ericsson S, Rudling M. Growth hormone and low-density lipoproteins. Acta Endocrinol 1993;128 Suppl 2:26-8.

63. Eden S, Wiklund O, Oscarsson J, Rosen T, Bengtsson BA. Growth hormone treatment of growth hormone-deficient adults results in a marked increase in $\mathrm{L} p(\mathrm{a})$ and HDL-C cholesterol concentrations. Arterioscler Thromb 1993;13:296-301.

64. Johansson JO, Fowelin J, Landin K, Lager I, Bengtsson BA. Growth hormone-deficient adults are insulinresistant. Metabolism 1995;44:1126-9.

65. Roman J, Villaizan CJ, Garcia-Foncillas J, Azcona C, Salvador J, Sierrasesumaga L. Chemotherapyinduced growth hormone deficiency in children with cancer. Med Pediatr Oncol 1995;25:90-5.

66. Sklar C, Whitton J, Mertens A, et al. Abnormalities of the thyroid in survivors of Hodgkin's disease: data from the Childhood Cancer Survivor Study. J Clin Endocrinol Metab 2000;85:3227-32.

67. Stava CJ, Jimenez C, Vassilopoulou-Sellin R. Endocrine sequelae of cancer and cancer treatments. J Cancer Surviv 2007;1:261-74.

68. Bowers DC, McNeil DE, Liu Y, et al. Stroke as a late treatment effect of Hodgkin's Disease: a report from the Childhood Cancer Survivor Study. J Clin Oncol 2005;23:6508-15.

69. Bowers DC, Liu Y, Leisenring W, et al. Late-occurring stroke among long-term survivors of childhood leukemia and brain tumors: a report from the Childhood Cancer Survivor Study. J Clin Oncol 2006;24:5277-82.

70. Oeffinger KC, Mertens AC, Sklar CA, et al. Chronic health conditions in adult survivors of childhood cancer. N Engl J Med 2006;355:1572-82.

71. Warner JT, Bell W, Webb DK, Gregory JW. Daily energy expenditure and physical activity in survivors of childhood malignancy. Pediatr Res 1998;43:607-13.

72. Reilly JJ, Ventham JC, Ralston JM, Donaldson M, Gibson B. Reduced energy expenditure in preobese children treated for acute lymphoblastic leukemia. Pediatr Res 1998;44:557-62.

73. Grundy SM. Drug therapy of the metabolic syndrome: minimizing the emerging crisis in polypharmacy. Nat Rev 2006;5:295-309.

74. Russell-Jones DL, Watts GF, Weissberger A, et al. The effect of growth hormone replacement on serum lipids, lipoproteins, apolipoproteins and cholesterol precursors in adult growth hormone deficient patients. Clin Endocrinol 1994;41:345-50.

75. Batsis JA, Nieto-Martinez RE, Lopez-Jimenez F. Metabolic syndrome: from global epidemiology to individualized medicine. Clin Pharmacol Ther 2007;82:509-24. 


\section{Abstract}

Background: Adult survivors of childhood cancer have been reported to have an increased risk of late sequels. A cluster of abnormalities that contribute to the metabolic syndrome may be expressed at a higher level and therefore result in an increased risk for diabetes mellitus and cardiovascular diseases.

Patients and methods: We investigated a single centre cohort of 500 adult survivors (228 females) of childhood cancer, median age 28 years (range 18-59 years) and median follow-up time 19 years (range $6-49$ years). We measured total cholesterol, high-density lipoproteincholesterol (HDL-C), systolic and diastolic blood pressure, body mass index and the prevalence of type 2 diabetes. Data from the epidemiologic MORGEN-study were used to calculate standard deviation scores as normative values.

Results: The criteria of the metabolic syndrome were met in $13 \%$ of the total cohort. Acute lymphoblastic leukemia (ALL) survivors treated with cranial irradiation had an increased risk of developing the metabolic syndrome compared to ALL survivors not treated with cranial irradiation ( $23 \%$ vs. $7 \%, \mathrm{P}=0.011$ ), probably determined by higher prevalence of overweight and hypertension.

Conclusion: Adult survivors of childhood cancer, especially those treated with cranial irradiation, are at increased risk of developing the metabolic syndrome. 


\section{Introduction}

During the last decades, the survival rate of childhood cancer has improved remarkably, resulting in an increasing number of survivors. ${ }^{1}$ Some of these survivors are now 30 or even more years after treatment. Children who survive cancer are at risk of developing late effects, as treatment is intensive and therapy is administered in a growing and developing individual. Various studies investigated the risk of second malignancies, cardiac problems, pulmonary complications, impaired fertility, problems of offspring, osteoporosis and obesity in survivors of childhood cancer. ${ }^{2-12}$ Mertens et al. found an increased standardized mortality ratio for cardiovascular diseases in survivors of several childhood malignancies. ${ }^{13}$ Obesity is a frequently reported late effect in long-term survivors of childhood acute lymphoblastic leukemia (ALL), especially when treatment included cranial irradiation. ${ }^{3,14}$ In addition, in survivors of other malignancies obesity has been reported. ${ }^{15,16}$ Several studies described a range of disorders like dyslipidemia, hypertension, hyperinsulinemia, impaired glucose tolerance and insulin resistance in long-term survivors after treatment for various malignancies. ${ }^{17-19}$ Together with obesity, these factors are in fact components of the metabolic syndrome.

The metabolic syndrome was first described by Reaven in $1988 .{ }^{20}$ He found a clustering of symptoms, including insulin resistance, hyperinsulinemia, glucose intolerance, elevated levels of triglycerides, a reduced high-density lipoprotein-cholesterol (HDL-C) level and hypertension, in patients and called this the Syndrome $X$. It was suggested to be an important risk factor for developing cardiovascular diseases and diabetes mellitus type II. Since then, many definitions of this metabolic syndrome were proposed by different organizations. ${ }^{21-26}$ Each definition contains at least the next components: insulin resistance or hyperglycaemia or hyperinsulinemia, in combination with obesity, hypertension and dyslipidemia. Other additional factors that are associated with the metabolic syndrome are a proinflammatory state, a prothrombotic state, hyperuricemia, hyperleptinemia and microalbuminuria. So far studies on the metabolic syndrome in large cohorts of childhood cancer survivors are not available. Therefore, we evaluated the metabolic syndrome in our cohort of childhood cancer survivors.

\section{Patients and methods}

\section{Patients}

Five-hundred adult survivors of childhood cancer, who were at least five years after cessation of therapy, treated in the Erasmus MC-Sophia Children's Hospital in Rotterdam, the Netherlands, were included. We created 11 subgroups according to type of cancer (Table 1). Patients were evaluated during their regular visit at the Late Effects Registration outpatient clinic (LATER-clinic). This evaluation included history, physical examination, laboratory tests and imaging studies. We compared the data on body mass index, lipid spectrum, blood pressure and prevalence of 
diabetes in childhood cancer survivors with the normal population, derived from the Monitoring van Risicofactoren en Gezondheid in Nederland (MORGEN) study, a large epidemiologic study performed in the Netherlands from 1993 to 1997. In this project, 10,219 males and 12,139 females aged 20 - 59 years were registered. ${ }^{27}$ In addition, we performed a separate analysis of patients who received cranial radiotherapy (CRT) $(N=62$, including one patient who received craniospinal radiotherapy) and compared them to patients who did not receive CRT (nonCRT) $(\mathrm{N}=354)$. Patients who received other types of radiotherapy were not included in this analysis, because of possible scatter radiation. One patient in the CRT group received $15 \mathrm{~Gy}$, five patients received $18-24 \mathrm{~Gy}$, and the other 58 patients received $\geq 24 \mathrm{~Gy}$. Moreover, we compared ALL survivors who received CRT ( $N=58$ ) to ALL survivors who did not receive CRT ( $N=104)$.

Table 1. Diagnosis groups.

\begin{tabular}{ll}
\hline Type of malignancy & Initial Diagnoses \\
\hline ALL & ALL \\
AML & AML \\
NHL & Burkitt, DLBCL, LCAL, NHL \\
HL & HL, HL paragranuloma \\
Bone tumours & Ewing, MFH Bone, Osteosarcoma \\
Wilms & Mesoblastoma, Wilms \\
Neuroblastoma & Neuroblastoma, GGLN, GNBL \\
LCH & LCH, Mal Hist \\
Germcell & Germcell \\
MMT & Chondrosarcoma, Chordosarcoma, Fibrous Histiocytoma, Fibrosarcoma, \\
& Leiomyosarcoma, MFH soft, MMT, MPNST, RMS, Sarcoma \\
Other & Carcinoid, CCSK, CML, Grawitz, Hepatoblastoma, Nasopharynxcarcinoma, \\
& Nephroblastomatosis, Retinal, Retinoblastoma
\end{tabular}

$\mathbf{A L L}=$ Acute Lymphoblastic Leukemia; $\mathbf{A M L}=$ Acute Myeloid Leukemia; NHL = Non Hodgkin Lymphoma; HL = Hodgkin's lymphoma; Wilms = Wilms tumour; $\mathbf{L C H}=$ Langerhans Cell Histiocytosis; Germcell = Germcell tumour; $\mathbf{M M T}=$ Malignant Mesenchymal Tumour; DLBCL = Diffuse Large B Cell Lymphoma; LCAL = Large Cell Anaplastic Lymphoma; MFH Bone = Malignant Fibriohistocytosis Histiocytoma; GGLN = Ganglioneuroma; GNBL = Ganglioneuroblastoma; Mal Hist = Malignant Histiocytosis; MFH soft = Malignant Histiocytosis; MMT = Malignant Mesenchymal Tumour; MPNST = Malignant Periferal Nerve Sheet Tumour; RMS = rhabdomyosarcoma; $\mathbf{C C S K}=$ Clear Cell Sarcoma of the Kidney; $\mathbf{C M L}$ adult = Chronic Myeloid Leukemia.

\section{Methods}

All data were collected from the database of the LATER outpatient clinic. Missing data were recruited from the medical records. Age and follow-up time were measured in years. Treatment was categorized by kind of chemotherapy, radiotherapy (dose and location), surgery or stem cell transplantation. Height was measured using a Harpenden stadiometer. Weight was 
measured on a standard clinical balance. The body mass index (BMI) was calculated as weight $(\mathrm{kg}) /\left(\right.$ height $\left.(\mathrm{m})^{2}\right)$. BMI $\geq 25 \mathrm{~kg} / \mathrm{m}^{2}$ was defined as overweight, $\mathrm{BMI} \geq 30 \mathrm{~kg} / \mathrm{m}^{2}$ was defined as obesity. ${ }^{28}$ Blood pressure was measured on the right arm of the patient with the Dinamap ${ }^{\circledR}$ Procare. Blood pressure levels were defined as hypertensive if either systolic blood pressure $\geq 140 \mathrm{mmHg}$ or diastolic blood pressure $\geq 90 \mathrm{mmHg} .{ }^{29}$ Presence of diabetes and family history of cardiovascular diseases and of hypercholesterolemia were routinely asked for during visits and were abstracted from the medical records. Total cholesterol and HDL-C were measured nonfasting according to the Hitachi 917 directive (Roche Diagnostica Germany). Insulin-like growth factor (IGF)-1 levels levels were assessed according to the Immunite 2000 directive (DPC, Los Angeles, NDA). IGF-1 levels below $15 \mathrm{nmol} / \mathrm{I}$ were considered abnormal. Unfortunately, it was not feasible to use the definitions of the metabolic syndrome according to the World Health Organization (WHO) ${ }^{23}$, National Cholesterol Education Program / Adult Treatment Panel III (NCEP/ ATPIII) ${ }^{21}$ or any of the other proposed definitions, ${ }^{22,24-26}$ because of the unavailability of fasting lipid spectrum and of fasting glucose in our patients. Therefore we decided to use the modified definition of the metabolic syndrome as reported by Haugnes et al. ${ }^{30}$ (Table 2).

Table 2. Modified definition of the metabolic syndrome according to Haugnes. ${ }^{30}$

\section{At least two of the following:}

Blood pressure $\geq 140 / 90 \mathrm{~mm} / \mathrm{Hg}$ or medication

Obesity: body mass index $\geq 30\left(\mathrm{~kg} /\left(\mathrm{m}^{2}\right)\right)$

Self reported prevalence of diabetes or medication

Serum total cholesterol $\geq 5.2 \mathrm{mmol} / \mathrm{l}$ or medication

\section{Statistics}

Statistical analysis was performed using the Statistical Package for Social Sciences (SPSS) 15.0. Non-parametric paired tests were used to compare (mean) standard deviation scores (SDS) values with healthy controls. Mann-Whitney $U$ test was used to compare the (mean) SDS values of CRT patients with nonCRT patients. Chi-square tests were used to compare categorical variables between CRT and nonCRT groups. P-values of $<0.05$ (two-tailed) were considered statistically significant.

\section{Results}

\section{Patients}

In this study 500 patients were included (228 females and 272 males). Patients were treated between 1958 and 2001 and visited the LATER-outpatient clinic between 2002 and 2007. Median 
age was 28 years (range $18-59$ years). Twenty-nine patients were younger than 20 and 25 patients were older than 40 at follow-up. Median follow-up time after cessation of therapy was 19 years (range $6-49$ years). Patient characteristics are shown in table 3.

Table 3. Diagnosis, sex distribution, age, age at diagnosis and follow-up time of the survivors.

\begin{tabular}{lrcrc}
\hline Diagnosis & $\begin{array}{c}\text { No. of subjects } \\
(\hat{\jmath} / \text { / })\end{array}$ & $\begin{array}{c}\text { Age } \\
\text { (yr) (range) }\end{array}$ & $\begin{array}{c}\text { Age at diagnosis } \\
\text { (yr) (range) }\end{array}$ & $\begin{array}{c}\text { Follow-up time } \\
\text { (yr) (range) }\end{array}$ \\
\hline All patients & $500(272 / 228)$ & $28(18-59)$ & $6(0-17)$ & $19(6-49)$ \\
ALL & $164(89 / 75)$ & $27(18-43)$ & $5(1-17)$ & $18(6-34)$ \\
AML & $22(13 / 9)$ & $28(19-38)$ & $10(0-14)$ & $19(9-27)$ \\
NHL & $61(42 / 19)$ & $28(20-41)$ & $9(2-16)$ & $18(6-31)$ \\
HL & $47(31 / 16)$ & $28(19-44)$ & $13(4-17)$ & $14(6-34)$ \\
Bone tumours & $29(15 / 14)$ & $30(19-46)$ & $12(1-16)$ & $17(8-42)$ \\
Wilms & $58(29 / 29)$ & $28(19-47)$ & $4(0-13)$ & $24(14-40)$ \\
Neuroblastoma & $36(14 / 22)$ & $27(18-43)$ & $1(0-12)$ & $24(11-38)$ \\
LCH & $13(3 / 10)$ & $27(19-37)$ & $2(1-9)$ & $23(14-29)$ \\
Germcell & $11(5 / 6)$ & $25(20-34)$ & $2(1-13)$ & $19(10-31)$ \\
MMT & $43(21 / 22)$ & $29(18-59)$ & $7(0-16)$ & $21(8-49)$ \\
Other & $16(10 / 6)$ & $27(20-35)$ & $1(0-15)$ & $20(10-33)$ \\
\hline
\end{tabular}

Values are medians (ranges). $\mathbf{A L L}=$ Acute Lymphoblastic Leukemia; $\mathbf{A M L}=$ Acute Myeloid Leukemia; $\mathbf{N H L}=$ Non Hodgkin Lymphoma; HL = Hodgkin's lymphoma; Wilms = Wilms tumour; $\mathbf{L C H}=$ Langerhans Cell Histiocytosis; Germcell = Germcell tumour; MMT = Malignant Mesenchymal Tumour; Other = Carcinoid; Clear Cell Sarcoma of the Kidney; Chronic Myeloid Leukemia; Grawitz; Hepatoblastoma; Nasopharynxcarcinoma; Nephroblastomatosis; Retinal; Retinoblastoma.

\section{Total cholesterol and HDL-cholesterol}

Compared to controls, total cholesterol was significantly increased in females (mean SDS 0.39, $\mathrm{P}<0.001$ ), but not in males (mean SDS $0.09, \mathrm{~ns}$ ). HDL-C was significantly increased in males compared to controls (mean SDS 0.29, P=0.009), but not in females (mean SDS 0.16, ns). Comparing cancer types, total cholesterol was increased in AML (mean SDS 0.94, $\mathrm{P}=0.001$ ), bone tumour (mean SDS 0.60, $\mathrm{P}=0.011$ ) and in MMT (mean SDS 0.40, $\mathrm{P}=0.035$ ) survivors. HDL-C was increased in NHL (mean SDS 0.48, P=0.021) and in Wilms tumour survivors (mean SDS 0.47, $\mathrm{P}=0.002$ ). (Table 4) No differences in total cholesterol or HDL-C were found between patients who received CRT or not. (Table 5 and 6) ALL survivors who received CRT had higher total cholesterol levels compared to ALL survivors who did not (mean SDS 0.38 vs. mean SDS $-0.05, P=0.027$ ), whereas their HDL-C levels did not differ. 


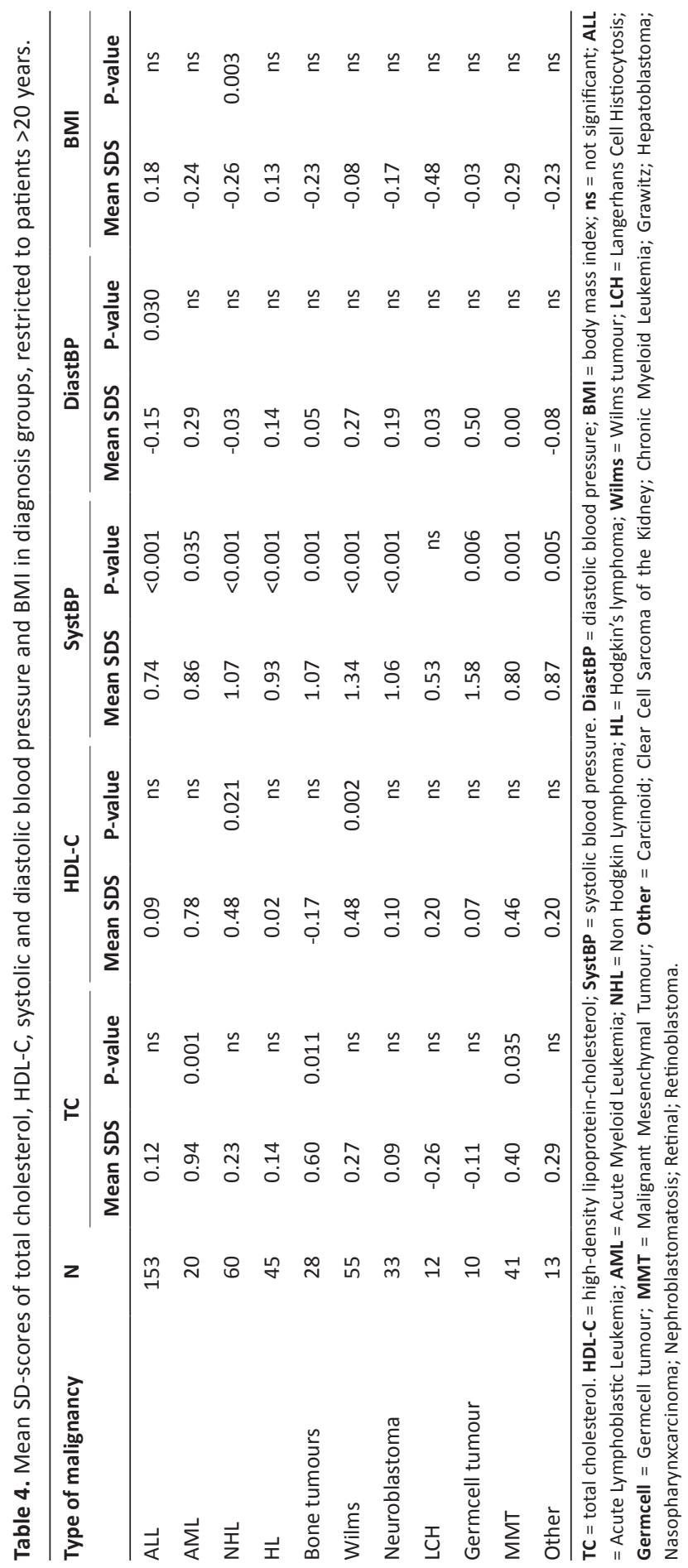




\section{Blood pressure}

Mean systolic blood pressure was significantly higher in males (mean SDS 0.80, P<0.001) and in females (mean SDS 1.11, $\mathrm{P}<0.001$ ) as compared to controls. Diastolic blood pressure was significantly higher in females (mean SDS 0.31, P<0.001), but lower in males (mean SDS 0.21, $\mathrm{P}<0.001$ ). Systolic blood pressure was increased in survivors of all cancer types, except for $\mathrm{LCH}$. Diastolic blood pressure was decreased in ALL survivors (Table 4). No differences in systolic or diastolic blood pressure were found between CRT and nonCRT groups (Table 5 and 6). However, ALL survivors treated with CRT were more often hypertensive compared to ALL survivors not treated with CRT (22\% vs. $10 \%, \mathrm{P}=0.036)$.

Table 5. SDS of total cholesterol, HDL-C, systolic and diastolic blood pressure and BMI in nonCRT and CRT group.

\begin{tabular}{lccc}
\hline & $\begin{array}{c}\text { nonCRT }(\mathbf{N}=354) \\
\text { Mean SDS (95\% Cl) }\end{array}$ & $\begin{array}{c}\text { CRT (N=64) } \\
\text { Mean SDS (95\% Cl) }\end{array}$ & P-value \\
\hline Total cholesterol & $0.13(0.02-0.25)$ & $0.33(0.03-0.63)$ & $\mathrm{ns}$ \\
HDL-C & $0.20(0.06-0.33)$ & $0.21(-0.15-0.57)$ & $\mathrm{ns}$ \\
SystBP & $0.94(0.80-1.08)$ & $0.78(0.48-1.08)$ & $\mathrm{ns}$ \\
DiastBP & $-0.01(-0.13-0.11)$ & $0.01(-0.26-0.28)$ & $\mathrm{ns}$ \\
BMI & $-0.10(-0.22-0.02)$ & $0.34(0.09-0.59)$ & $<0.001$ \\
\hline
\end{tabular}

Restricted to patients not treated with bone marrow transplantation and patients not treated with radiotherapy other than cranial. CRT = survivors treated with cranial irradiation; nonCRT = survivors not treated with cranial irradiation; HDL-C = highdensity lipoprotein-cholesterol; SystBP = systolic blood pressure; DiastBP = diastolic blood pressure; BMI = body mass index; $\mathbf{n s}=$ not significant; $\mathbf{B M I}=$ body mass index; $\mathbf{C l}$ = confidence interval.

\section{Body mass index}

BMI was not different from controls in females (mean SDS 0.10, ns), and was significantly decreased in males (mean SDS $-0.17, \mathrm{P}<0.001$ ). Within the specific cancer types, BMI was not different from controls. Survivors treated with CRT, compared to survivors not treated with CRT, had a significantly higher BMI (mean SDS difference 0.44, $\mathrm{P}<0.001$ ) (Table 5). Male survivors treated with CRT were more often overweight ( $59 \%$ vs. $28 \%, \mathrm{P}=0.001$ ) and more often obese (13\% vs. $2.0 \%, P=0.015)$. Female survivors treated with CRT were more often overweight (55\% vs. $27 \%, \mathrm{P}=0.004$ ), but not more often obese. (Table 6) Additionally, survivors treated with CRT more frequently had low IGF-1 levels compared to survivors not treated with CRT ( $29 \%$ vs. $5 \%$, $\mathrm{P}<0.001)$. Moreover, ALL survivors treated with CRT, compared to ALL survivors not treated with $\mathrm{CRT}$, had a significantly increased BMI (mean SDS difference 0.34, $\mathrm{P}=0.014$ ). ALL survivors treated with CRT were more often overweight compared to ALL survivors not treated with CRT (59\% vs. $34 \%, \mathrm{P}=0.003)$, but not more often obese ( $12 \%$ vs. $9 \%, \mathrm{~ns})$. ALL survivors treated with CRT more frequently had low IGF-1 levels compared to ALL survivors not treated with CRT (32\% vs. $8 \%$, $\mathrm{P}=0.003)$. 
Table 6. Percentages of survivors with hypercholesterolemia, decreased HDL-C levels, hypertension, overweight, obesity, diabetes and metabolic syndrome in nonCRT group compared to CRT group.

\begin{tabular}{|c|c|c|c|}
\hline Variable & nonCRT & CRT & P-value \\
\hline \multicolumn{4}{|c|}{ Hypercholesterolemia } \\
\hline$\delta$ & $3 \%$ & $9 \%$ & ns \\
\hline 우 & $6 \%$ & $13 \%$ & ns \\
\hline \multicolumn{4}{|c|}{ Low HDL-C } \\
\hline$\hat{0}$ & $11 \%$ & $19 \%$ & ns \\
\hline q & $2 \%$ & $7 \%$ & ns \\
\hline \multicolumn{4}{|c|}{ Hypertension } \\
\hline$\delta$ & $20 \%$ & $28 \%$ & ns \\
\hline q & $14 \%$ & $16 \%$ & ns \\
\hline \multicolumn{4}{|c|}{ Overweight } \\
\hline 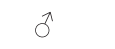 & $28 \%$ & $59 \%$ & 0.001 \\
\hline q & $27 \%$ & $55 \%$ & 0.004 \\
\hline \multicolumn{4}{|l|}{ Obesity } \\
\hline$\hat{\sigma}$ & $2 \%$ & $13 \%$ & 0.015 \\
\hline q & $12 \%$ & $10 \%$ & ns \\
\hline \multicolumn{4}{|l|}{ Diabetes } \\
\hline$\hat{\sigma}$ & $0 \%$ & $0 \%$ & n.a. \\
\hline q & $1 \%$ & $0 \%$ & ns \\
\hline \multicolumn{4}{|c|}{ Prevalence of the metabolic syndrome } \\
\hline$\partial$ & $7 \%$ & $25 \%$ & 0.003 \\
\hline 우 & $12 \%$ & $20 \%$ & ns \\
\hline
\end{tabular}

Restricted to patients not treated with bone marrow transplantation and patients not treated with radiotherapy other than cranial. HDL-C = high-density lipoprotein-cholesterol; $\mathbf{C R T}=$ survivors treated with cranial irradiation; nonCRT = survivors not treated with cranial irradiation; $\mathbf{n s}=$ not significant; $\mathbf{n} . \mathbf{a}$. = not available.

\section{Type 2 diabetes}

In our patient group, three out of 500 (0.6\%) survivors had self-reported diabetes mellitus. One of them was 19 years old, diagnosed with chronic myeloid leukemia at the age of one year, treated with chemotherapy, radiotherapy and bone marrow transplantation. The other two were 24 and 29 years old, both survivors of Wilms tumour. They were both treated with surgery and chemotherapy, the latter also with radiotherapy. None of the diabetic patients had received cranial irradiation or prednisone therapy. Body mass indexes of these patients were respectively $18 \mathrm{~kg} /\left(\mathrm{m}^{2}\right), 24 \mathrm{~kg} /\left(\mathrm{m}^{2}\right)$ and $31 \mathrm{~kg} /\left(\mathrm{m}^{2}\right)$. The prevalence of diabetes in the patients seemed to be the same as in the normal population (Table 6). 


\section{Combining components of the metabolic syndrome}

Scoring of components of the metabolic syndrome was performed according to the definition of Haugnes $^{30}$ (Table 2), which indicates subjects with at least two components as positive. In 466 survivors information on all required parameters was available. Thirteen percent $(\mathrm{N}=59)$ of the survivors had at least two components (males 12\%, females $14 \%$ ) of the metabolic syndrome. Two percent had three components and in only one person $(0.2 \%)$ four components were determined. Of the 59 survivors with $\geq 2$ components of the metabolic syndrome, 52 were younger than 40 years.

Prevalence of the metabolic syndrome was higher in male CRT patients than in male nonCRT patients ( $25 \%$ vs. $7 \%, \mathrm{P}=0.003$ ), but not in female CRT patients compared to female nonCRT patients ( $18 \%$ vs. $12 \%$, ns) (Table 6). Prevalence of the metabolic syndrome was higher in ALL survivors treated with CRT compared to ALL survivors not treated with CRT ( $23 \%$ vs. $7 \%, P=0.011$ ).

\section{Discussion}

We found that long-term adult survivors of childhood cancer, especially those treated with CRT, are at increased risk of developing components of the metabolic syndrome. The present analysis of 500 adult survivors of childhood cancer provides information on the occurrence of components of the metabolic syndrome in long-term survivors of 11 types of childhood cancer, using a large epidemiologic control group. Although several childhood cancer survivor studies have also shown the possible relationship between treatment and an increased risk of developing risk factors contributing to the metabolic syndrome, studies have been limited by small sample sizes and lack of optimal comparison groups. Additionally, most studies assessed only one or some determinants of the metabolic syndrome instead of the full content of the syndrome, or focused on only one type of cancer.

Our study shows that total cholesterol levels were elevated in females, in contrast to in males. This was not due to increased HDL-C. Therefore, it seems likely that the increased cholesterol in females can be explained by LDL-C or very low-density lipoproteins. These lipoproteins are known to cause atherosclerosis and therefore increase the risk of cardiovascular diseases. Why male survivors have higher HDL-C levels, which may be advantageous, is unclear. Additionally, survivors of ALL treated with CRT had increased total cholesterol levels (mean SDS 0.38 vs. mean SDS -0.05, $P=0.027$ ) compared to their counterparts not treated with CRT. Dyslipidemia has been reported in survivors of ALL, ${ }^{3,14}$ brain cancer, ${ }^{31}$ stem cell transplantation ${ }^{19}$ and in heterogeneous groups of survivors of childhood cancer. ${ }^{18,32}$ However, besides decreased HDL-C levels, elevated LDL-C levels and/or elevated triglyceride levels characterize dyslipidemia. Unfortunately LDL-C and triglycerides were not available in this survey. This indicates that the contribution of dyslipidemia may even be higher than we indirectly observed by total cholesterol and HDL-C measurement. 
In our study we found increased systolic blood pressure both in men and women. Systolic blood pressure was increased after treatment for all types of malignancies, except for LCH-C (Table 4). Systolic and diastolic blood pressures were both significantly correlated with BMI and hypertensive survivors were more often overweight than survivors with normal blood pressure. Additionally, ALL survivors treated with CRT were more often hypertensive than ALL survivors not treated with CRT, which might be explained by a relatively stronger correlation between systolic and diastolic blood pressure and BMI in this subgroup. A previous study did not find a significant difference in blood pressure between 50 survivors of childhood cancer (excluding brain cancer) at a median follow-up time of 13 years, and controls. However, they found a higher systolic blood pressure in a risk group, which they defined by the combination of obesity, hyperinsulinemia and low HDL-C levels. ${ }^{32}$ Furthermore, hypertension has been reported in $13-19 \%$ in studies that included 103 and 124 adult survivors of a variety of malignancies respectively. ${ }^{17,18}$ It is unknown what determines the elevated blood pressure in survivors. Dysfunction of the endothelium has been hypothesized to be the initial step in the development of cardiovascular diseases. Chemotherapy agents like anthracyclines are known to damage the vascular endothelium. Additionally, radiotherapy could have a damaging effect on the endothelium.

We found BMI not to be increased significantly, neither in males or females. As BMI is not representing the exact distribution of fat, it is possible that measuring abdominal obesity by measuring the waist circumference would have identified abdominal adiposity more accurately. This is illustrated by the fact that normal body mass indexes together with increased abdominal obesity have been reported in ALL survivors. ${ }^{3,4}$ It is advisable to measure waist circumference and lean body mass in future studies. The survivors treated with CRT had a significantly higher BMI compared to nonCRT survivors. Male and female survivors were more often overweight and males also more often obese compared to the normal population. Male ALL survivors treated with CRT were more often overweight than male ALL survivors not treated with CRT, however they were not more often obese. Female ALL survivors treated with CRT were not more often overweight or obese. These results are in contrast to those of Oeffinger et al., ${ }^{12}$ who reported a higher incidence of obesity in female ALL survivors treated with CRT $\geq 20$ Gy, than in males and than in normal controls. It should be noticed that Oeffinger et al. used self-reported BMI values, whereas we actually measured weight and height. Obesity has been reported frequently in survivors treated with $\mathrm{CRT}^{4,15,32,33}$ in which growth hormone deficiency is suggested to play a causal role. Growth hormone deficiency may be caused by direct pituitary damage resulting from CRT, but also by hypothalamic damage. Although a low IGF-1 level is a reasonable indicator of growth hormone deficiency, a normal IGF-1 will not rule out growth hormone deficiency. For this a dynamic assessment of growth hormone is necessary. It should however be considered that these tests are more invasive for the survivors.

Only three out of 500 survivors had diabetes, i.e. two survivors of Wilms tumour and one survivor of chronic myeloid leukemia. Insulin resistance, in which a given concentration of insulin is associated with a subnormal glucose response, has been described in adult survivors of several 
malignancies. ${ }^{3,14,18,19,32}$ Therefore, it is important to investigate insulin resistance in the future in larger prospective cohorts, as well as parameters like pro-thrombotic and pro-inflammatory factors, hyperuricemia, hyperleptinemia and microalbuminuria, which are also reported to be associated with the occurrence of the metabolic syndrome.

Besides the size, the strength of the current study is the availability of the unique control cases of the epidemiologic MORGEN-study in the Netherlands. This enabled the comparison of BMI, total cholesterol and HDL-C, blood pressure and the prevalence of type 2 diabetes in childhood cancer survivors with the normal population. However, although the MORGENstudy measured the single components of the metabolic syndrome, no direct estimation of the prevalence of the metabolic syndrome was provided. In our cohort, $59(13 \%)$ survivors had the metabolic syndrome. Interestingly, $52(12 \%)$ of these survivors were younger than 40 years. In several European countries a prevalence of the metabolic syndrome varying between $7-36 \%$ (male) and $5-22 \%$ (female) aged $40-55$ years has been described. ${ }^{24}$ Studies in $20-40$ years old normal people are unfortunately not available. The fact that $12 \%$ of the survivors younger than 40 already met the criteria for the metabolic syndrome, suggests that the metabolic syndrome may play an important role in our survivors. In the normal population, the prevalence of the metabolic syndrome increases with age,$^{34}$ indicating that this may reflect an emerging problem in the future. This indicates that screening may be useful to detect disguised metabolic syndrome, in order to develop preventive strategies.

We found an increased prevalence of the metabolic syndrome in CRT patients (23\%) compared to nonCRT patients $(9 \%)$. When looking closer, this difference was only significant in males but not in females. Similarly to what Trimis et al..$^{33}$ found, prevalence of the metabolic syndrome was higher in ALL survivors treated with CRT compared to ALL survivors not treated with CRT ( $23 \%$ vs. 7\%).

In conclusion, childhood cancer survivors, especially those treated with CRT, are at greater risk of having components of the metabolic syndrome. Prospective studies, with matched controls (siblings), are urgently needed to evaluate the risk for cardiovascular diseases and type 2 diabetes based on up to date determinants of the metabolic syndrome, and, if necessary, to design intervention strategies. Therefore, it is necessary to start screening for components of the metabolic syndrome in childhood cancer survivors, in order to prevent cardiovascular events and development of type 2 diabetes. 


\section{References}

1. Meadows AT. Pediatric cancer survivors: Past history and future challenges. Curr Probl Cancer 2003; 27: 112-126.

2. Robison LL, Green DM, Hudson M et al. Long-term outcomes of adult survivors of childhood cancer. Cancer 2005; 104: 2557-2564.

3. Jarfelt M, Lannering B, Bosaeus I et al. Body composition in young adult survivors of childhood acute lymphoblastic leukemia. Eur J Endocrinol 2005; 153: 81-89.

4. Gurney JG, Ness KK, Sibley SD et al. Metabolic syndrome and growth hormone deficiency in adult survivors of childhood acute lymphoblastic leukemia. Cancer 2006; 107: 1303-1312.

5. van Casteren NJ, van der Linden GH, Hakvoort-Cammel FG et al. Effect of childhood cancer treatment on fertility markers in adult male long-term survivors. Pediatr Blood Cancer 2009; 52: 108-112.

6. van Beek RD, de Muinck Keizer-Schrama SM, Hakvoort-Cammel FG et al. No difference between prednisolone and dexamethasone treatment in bone mineral density and growth in long term survivors of childhood acute lymphoblastic leukemia. Pediatr Blood Cancer 2006; 46: 88-93.

7. van Beek RD, Smit M, van den Heuvel-Eibrink MM et al. Inhibin B is superior to FSH as a serum marker for spermatogenesis in men treated for Hodgkin's lymphoma with chemotherapy during childhood. Hum Reprod 2007; 22: 3215-3222.

8. van Beek RD, van den Heuvel-Eibrink MM, Laven JS et al. Anti-Mullerian hormone is a sensitive serum marker for gonadal function in women treated for Hodgkin's lymphoma during childhood. J Clin Endocrinol Metab 2007; 92: 3869-3874.

9. van der Sluis IM, van den Heuvel-Eibrink MM, Hahlen K et al. Bone mineral density, body composition, and height in long-term survivors of acute lymphoblastic leukemia in childhood. Med Pediatr Oncol 2000; 35: 415-420.

10. Lie Fong S, Laven JS, Hakvoort-Cammel FG et al. Assessment of ovarian reserve in adult childhood cancer survivors using anti-Mullerian hormone. Hum Reprod 2009; 24: 982-990.

11. van Beek RD, van den Heuvel-Eibrink MM, Hakvoort-Cammel FG et al. Bone mineral density, growth and thyroid function in long-term survivors of pediatric Hodgkin's lymphoma treated with chemotherapy only. J Clin Endocrinol Metab 2009 Jun;94(6):1904-9.

12. Oeffinger KC, Mertens AC, Sklar CA et al. Obesity in adult survivors of childhood acute lymphoblastic leukemia: a report from the Childhood Cancer Survivor Study. J Clin Oncol 2003; 21: 1359-1365.

13. Mertens AC. Cause of mortality in 5-year survivors of childhood cancer. Pediatr Blood Cancer 2007; 48: 723-726.

14. Oeffinger KC, Buchanan GR, Eshelman DA et al. Cardiovascular risk factors in young adult survivors of childhood acute lymphoblastic leukemia. J Pediatr Hematol Oncol 2001; 23: 424-430.

15. Hoffman KE, Derdak J, Bernstein D et al. Metabolic syndrome traits in long-term survivors of pediatric sarcoma. Pediatr Blood Cancer 2008; 50: 341-346.

16. Nysom K, Holm K, Michaelsen KF et al. Degree of fatness after treatment of malignant lymphoma in childhood. Med Pediatr Oncol 2003; 40: 239-243.

17. Haddy TB, Mosher RB, Reaman GH. Hypertension and prehypertension in long-term survivors of childhood and adolescent cancer. Pediatr Blood Cancer 2007; 49: 79-83.

18. Neville KA, Cohn RJ, Steinbeck KS et al. Hyperinsulinemia, impaired glucose tolerance, and diabetes mellitus in survivors of childhood cancer: prevalence and risk factors. J Clin Endocrinol Metab 2006; 91: 4401-4407.

19. Taskinen M, Saarinen-Pihkala UM, Hovi L, Lipsanen-Nyman M. Impaired glucose tolerance and dyslipidemia as late effects after bone-marrow transplantation in childhood. Lancet 2000; 356: 993997.

20. Reaven GM. Banting lecture 1988. Role of insulin resistance in human disease. Diabetes 1988; 37 : 1595-1607. 
21. Executive Summary of The Third Report of The National Cholesterol Education Program (NCEP) Expert Panel on Detection, Evaluation, And Treatment of High Blood Cholesterol In Adults (Adult Treatment Panel III). JAMA 2001; 285: 2486-2497.

22. Alberti KG, Zimmet P, Shaw J. Metabolic syndrome - a new world-wide definition. A Consensus Statement from the International Diabetes Federation. Diabet Med 2006; 23: 469-480.

23. Alberti KG, Zimmet PZ. Definition, diagnosis and classification of diabetes mellitus and its complications. Part 1: diagnosis and classification of diabetes mellitus provisional report of a WHO consultation. Diabet Med 1998; 15: 539-553.

24. Balkau B, Charles MA, Drivsholm T et al. Frequency of the WHO metabolic syndrome in European cohorts, and an alternative definition of an insulin resistance syndrome. Diabetes Metab 2002; 28 : 364-376.

25. Einhorn D, Reaven GM, Cobin RH et al. American College of Endocrinology position statement on the insulin resistance syndrome. Endocr Pract 2003; 9: 237-252.

26. Grundy SM, Cleeman JI, Daniels SR et al. Diagnosis and management of the metabolic syndrome: an American Heart Association/National Heart, Lung, and Blood Institute Scientific Statement. Circulation 2005; 112: 2735-2752.

27. A. Blokstra HAS, H.B. Bueno de Mesquita,, J.C. Seidell WMMV. Monitoring van Risicofactoren en Gezondheid in Nederland (MORGEN-project) 1993-1997. Leefstijl- en risicofactoren: prevalenties en trends. 1997.

28. Obesity: preventing and managing the global epidemic. Report of a WHO consultation. World Health Organ Tech Rep Ser 2000; 894: i-xii, 1-253.

29. Chobanian AV, Bakris GL, Black HR et al. Seventh report of the Joint National Committee on Prevention, Detection, Evaluation, and Treatment of High Blood Pressure. Hypertension 2003; 42: 1206-1252.

30. Haugnes HS, Aass N, Fossa SD et al. Components of the metabolic syndrome in long-term survivors of testicular cancer. Ann Oncol 2007; 18: 241-248.

31. Heikens J, Ubbink MC, van der Pal HP et al. Long term survivors of childhood brain cancer have an increased risk for cardiovascular disease. Cancer 2000; 88: 2116-2121.

32. Talvensaari KK, Lanning M, Tapanainen P, Knip M. Long-term survivors of childhood cancer have an increased risk of manifesting the metabolic syndrome. J Clin Endocrinol Metab 1996; 81: 3051-3055.

33. Trimis G, Moschovi M, Papassotiriou I et al. Early indicators of dysmetabolic syndrome in young survivors of acute lymphoblastic leukemia in childhood as a target for preventing disease. J Pediatr Hematol Oncol 2007; 29: 309-314.

34. Day C. Metabolic syndrome, or What you will: definitions and epidemiology. Diab Vasc Dis Res 2007; 4: 32-38. 


\section{Abstract}

Background: Aim of this study was to investigate the long-term endocrine effects of treatment for childhood non-Hodgkin Lymphoma (NHL).

Patients and methods: A single centre cohort of 84 survivors (22 females) was included in this retrospective study. Median age was 21 yrs ( $9-40$ yrs) and time after cessation of therapy 12 yrs (4 - 30 yrs). Height, weight, percentage fat, lean body mass (LBM), bone mineral content (BMC), bone mineral density of total body $\left(\mathrm{BMD}_{\mathrm{TB}}\right)$ and lumbar spine $\left(\mathrm{BMD}_{\mathrm{LS}}\right)$ were measured. Thyroid stimulating hormone (TSH), free thyroxin (fT4), insulin-like growth factor-1 (IGF-1), Inhibin-B and anti-müllerian hormone (AMH) levels were measured. Results were compared with Dutch controls.

Results: Height was lower in survivors (mean SDS -0.36, P=0.002), but further analysis showed that shorter stature was already present at diagnosis (mean SDS $-0.28, \mathrm{P}=0.023$ ). $\mathrm{BMI}$, percentage fat, $B M C, B M D_{T B}$ and $B M D_{L S}$ were not different from controls. $L B M$ was lower in survivors (mean SDS $-0.47, P=0.008$ ). TSH, $\mathrm{fT} 4$ and IGF-1 were normal in all survivors. $3 / 20$ adult females had low AMH levels and 23/42 adult males had low Inhibin-B levels.

Conclusions: Twelve years after cessation of treatment, NHL survivors did not develope adiposity, osteoporosis or thyroid disease. Male survivors may be at risk for infertility. 


\section{Introduction}

Six percent of children with a malignancy suffer from non Hodgkin lymphomas (NHL). ${ }^{1}$ Currently, the 5 -year survival for childhood $\mathrm{NHL}$ is $\sim 80 \%$. Because of this high survival rate and since treatment is administered in a growing and developing individual, long-term side effects are an important issue. Treatment for NHL consists of intensive chemotherapy, in rare cases combined with radiotherapy. Treatment schedules involve high doses of corticosteroids, which may cause reduced bone mineral density, osteoporosis and obesity. ${ }^{2}$ Both normal and decreased bone mineral density (BMD) have been reported in long-term survivors of other malignancies treated with chemotherapy including corticosteroids. ${ }^{3-9}$ In addition, altered body composition, especially obesity, was observed during and after treatment of acute lymphoblastic leukemia (ALL). ${ }^{10-19}$ Other endocrine or metabolic sequelae that have been described in NHL and ALL survivors are growth hormone deficiency, hypothyroidism, insulin resistance and dyslipidemia. ${ }^{18-20}$ Moreover, infertility or impaired reproductive outcome has been described in survivors of childhood cancer. ${ }^{21-25}$

So far, studies on endocrine long-term effects in survivors of childhood NHL are scarce and often limited by small sample sizes, or by the fact that data of NHL survivors have been combined with that of survivors of other malignancies. ${ }^{20,26-31}$ Therefore, we investigated endocrine late sequelae after childhood NHL in a single center cohort.

\section{Patients and methods}

\section{Patients}

We identified all survivors who were alive and $\geq 4$ years after cessation of therapy on January $1^{\text {st }} 2009$ and were treated for childhood non B-NHL, B-cell type lymphoma (B-NHL) or Large Cell Anaplastic Lymphoma (LCAL) at the Erasmus Medical Centre-Sophia Children's Hospital from 1975 - 2003. Survivors that visited the LATe Effects Registration (LATER)-outpatient clinic were eligible for analysis. The local ethical committee approved the study and consent according to the Helsinki declaration was obtained..$^{32}$ Outpatient clinic visits and performance of dual-energy X-ray absorptiometry (DXA) scans took place between July 2003 and November 2008. As they received similar treatment regimens, the LCAL survivors were analyzed together with the B-NHL group. Baseline characteristics and treatment details of survivors are summarized in Table 1.

\section{Anthropometry}

Height was measured using a Harpenden stadiometer and compared with Dutch normative values. $^{33}$ Height of survivors of other ethnicities was compared with normative values for these ethnicities. ${ }^{34,35}$ No data on target height were available. Weight was measured on a standard clinical balance. Body mass index (BMI) was calculated as weight $(\mathrm{kg}) /\left(\right.$ height $\left.(\mathrm{m})^{2}\right)$. 
$\mathrm{BMI}$ of survivors younger than 20 years was compared with Dutch normative values for this age group. ${ }^{33} \mathrm{BMI}$ of survivors of other ethnicities was compared with normative values for these ethnicities. ${ }^{34,35} \mathrm{BMI}$ of survivors aged 20 years and older were compared with Dutch normative values of the MOnitoring van Risicofactoren en GEzondheid in Nederland (MORGEN) study, a large epidemiologic study conducted in the Netherlands between 1993-1997, in which 10,219 males and 12,139 females aged 20 - 59 years were registered. ${ }^{36}$ Reference values of subjects aged 20-40 years in the MORGEN-study, i.e. 9,126 subjects (5,103 females), were used because all our survivors were younger than 40 years.

\section{Body composition and bone mineral density}

Bone mineral density of total body $\left(B M D_{T B}\right)$ and lumbar spine $\left(B M D_{L S}\right)$ were measured using dualenergy X-ray absorptiometry (DXA)-scans (Lunar Prodigy, Madison, WI, USA). To correct for bone size, we calculated bone mineral apparent density of the lumbar spine $\left(B M A D_{L S}\right)$, as described by Kröger et al. ${ }^{37} \mathrm{~A}$ DXA-scan of the total body also assesses body composition, i.e. lean body mass (LBM) in grams, percentage fat and bone-mineral content (BMC) in grams.

The results of survivors that were younger than 20 years were compared with those for a control group consisting of 444 healthy children and young adults ( 256 females) measured on the Lunar DPX-L device. ${ }^{38}$ Because outcomes of Lunar Prodigy and Lunar DPX-L can differ, a formula, derived from simultaneous measurements on Prodigy and DPXL in healthy individuals, was used to enable comparison of outcomes. The results for the survivors that are aged $\geq 20$ years were compared with those for a control group consisting of healthy young adults (58 males and 83 females; median age 23 years (range $18-37$ years), who were measured on the Lunar Prodigy device. $^{3}$

\section{Laboratory measurements}

All blood samples were taken during regular consultation at the LATER-outpatient clinic. Blood samples were processed within $2 \mathrm{~h}$ after withdrawal, and serum was stored at $-20^{\circ} \mathrm{C}$ until assay. Free thyroxin (fT4) levels were measured by chemoluminescence assays (Vitros $\mathrm{ECl}$ immunodiagnostic system, Ortho Diagnostics, Rochester, NY, USA). Normal values, determined in our laboratory were $11-25 \mathrm{pmol} / \mathrm{l}$ (interassay VC\% 4.7\% - 5.4\%) for fT4. The normal values for thyroid stimulating hormone (TSH) are $0.4-4.3 \mathrm{mU} / \mathrm{I}$ (interassay VC\% $2.5-4.1 \%$ ). Insulin-like growth factor-1 (IGF-1) and thyroid stimulating hormone (TSH) levels were assessed according to the Immulite 2000 (Diagnostic Products Corp., Los Angeles, CA, USA). Age adjusted normal values for IGF-1 were used to calculate SDS, according to Elmlinger et al. ${ }^{39}$ Anti-müllerian hormone $(\mathrm{AMH})$ levels $<1.0 \mu \mathrm{g} / \mathrm{l}$ are considered abnormal. AMH was measured using an in-house double-antibody enzyme-linked immunosorbent assay (ELISA), intra- and inter-assay coefficients of variance were $<10 \%$ and $<5 \% .{ }^{40,41}$ The reference values of follicle stimulating hormone (FSH) and Inhibin B for male adults in our institute are $2.0-7.0 \mathrm{U} / \mathrm{L}$ and $150-400 \mathrm{ng} / \mathrm{L}$, respectively. 


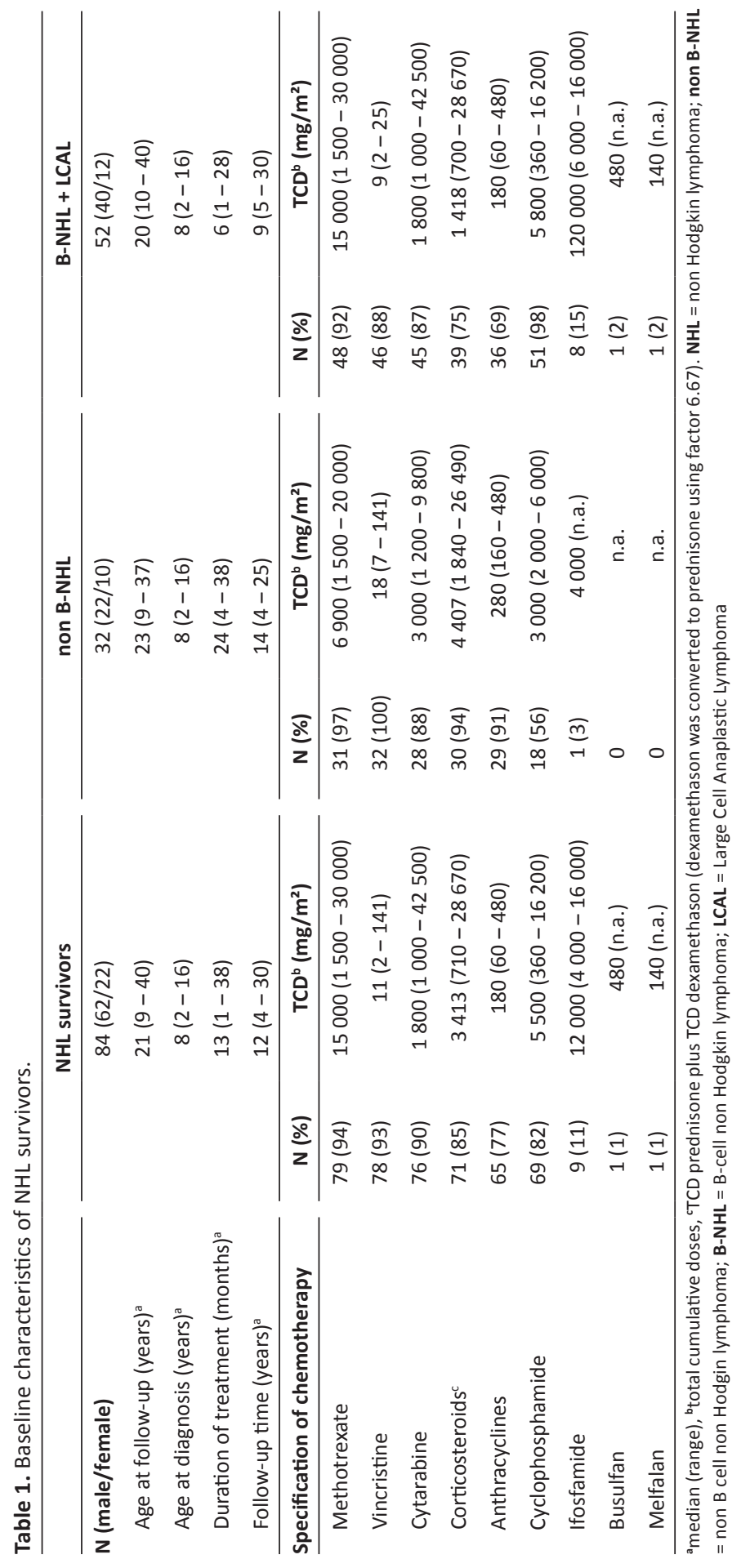


Within-assay and between-assay coefficients of variation (CV) for FSH were $5 \%$ and $11 \%$ and for Inhibin B 9\% and 15\%. ${ }^{42}$ The cut-off value of $150 \mathrm{ng} / \mathrm{L}$ for Inhibin B is taken as it has been shown that this provided the highest sensitivity and specificity in identifying low semen quality. ${ }^{43}$

\section{Statistics}

Statistical analysis was performed using the Statistical Package for Social Sciences (SPSS) 15.0 (SPSS, Inc., Chicago, IL, USA). Values are expressed as standard deviation scores (SDS). One sample T-test was used to compare mean SDS values of the survivors with healthy controls. Independent sample T-test was used to compare mean SDS values between survivor subgroups. Linear regression analysis was performed to assess the influence of age at follow-up, sex, type of NHL and LBM on height, and to assess the influence of age at diagnosis, alkylating agent dose and cytarabine dose on Inhibin B levels in adult males. Height SDS and Inhibin B levels were normally distributed. Linear regression was performed with a maximum of three to four dependent variables, because of the small (sub)group size. Changes in height from diagnosis until ten years after diagnosis were analyzed using repeated measurements analysis (SAS PROC MIXED SAS Institute, Inc., Cary, NC, USA), with an unstructured repeated covariance type. P-values less than 0.05 (two-sided) were considered statistically significant.

\section{Results}

\section{Patients}

Ninety-two survivors long-term NHL survivors were identified, of whom 84 visited the outpatient clinic and could be analyzed (Figure 1). Sixty-two males and 22 females were identified, consistent with the incidence of NHL (NHL occurs two to three times as often in males as in females). Thirtytwo of the 84 survivors were diagnosed non B-NHL, 46 B-NHL, and 6 LCAL. 76 survivors were Dutch, 4 Moroccan, 3 Turkish and 1 Asian. Median age at diagnosis was 8 years (range $2-16$ yrs), and at follow-up 21 years (range $9-40$ yrs). Thirty-six subjects (six females) were aged $<20$ years at follow-up.

\section{Anthropometry}

Height and weight measurements were available in all survivors $(\mathrm{N}=84)$. BMI was within the normal range in survivors and height was lower compared with controls (Table 2). Males and females were not different with respect to BMI (mean SDS -0.01 vs. $0.21, P=0.434$ ) or height (mean SDS -0.43 vs. $-0.16, P=0.279$ ), however male survivors reached a lower height compared with controls (mean SDS -0.43, P=0.002). Non B-NHL survivors and B-NHL/LCAL survivors were not different with respect to $\mathrm{BMI}$ (mean SDS -0.08 vs. $0.13, \mathrm{P}=0.409$ ) and height (mean SDS -0.41 vs. mean SDS $-0.32, \mathrm{P}=0.705$ ). $\mathrm{BMI}$ and height of six patients treated with radiotherapy (including two patients who were treated with bone marrow transplantation) were not different from those 
of Dutch normative values. In linear regression analysis with height (SDS) as dependent variable, age at follow-up, sex and type of NHL were not significant (Table 3). We additionally performed a longitudinal analysis to investigate height at different time points; namely at diagnosis, six months after diagnosis, and one year, three years, five years and ten years after diagnosis. Interestingly, height was already significantly lower in NHL survivors as compared with healthy controls at diagnosis (mean SDS $-0.28, \mathrm{P}=0.023$ ) and continued to be low at all investigated time points $(\mathrm{P}<0.001)$.

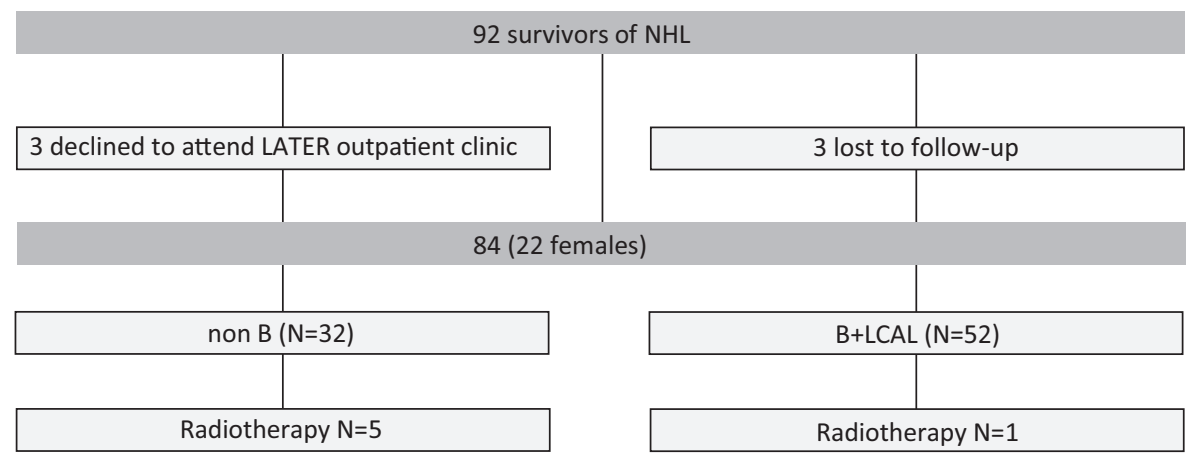

Figure 1. Recruitment of non-Hodgkin lymphoma survivors.

NHL = non-Hodgkin lymphoma; non B = non B cell non-Hodgin lymphoma; $\mathbf{B}=$ B-cell non-Hodgkin lymphoma; $\mathbf{L C A L}=$ Large Cell Anaplastic Lymphoma

Table 2. Anthropometry, body composition and bone mineral density in NHL survivors.

\begin{tabular}{|c|c|c|c|}
\hline & Mean SDS & 95\% C.I. ${ }^{a}$ & P-value ${ }^{b}$ \\
\hline \multicolumn{4}{|c|}{ All survivors ${ }^{c}$} \\
\hline $\mathrm{BMI}^{\mathrm{d}}$ & 0.05 & $-0.20,0.29$ & 0.323 \\
\hline Height & -0.36 & $-0.58,-0.14$ & 0.002 \\
\hline \multicolumn{4}{|c|}{ Survivors with a DXA-scan available ${ }^{e}$} \\
\hline $\mathrm{BMI}^{\mathrm{d}}$ & 0.03 & $-0.25,0.31$ & 0.849 \\
\hline Height & -0.34 & $-0.62,-0.05$ & 0.022 \\
\hline$\%$ fat & 0.19 & $-0.05,0.43$ & 0.111 \\
\hline $\operatorname{LBM}^{f}$ & -0.47 & $-0.81,-0.13$ & 0.008 \\
\hline $\mathrm{BMC}^{\mathrm{g}}$ & -0.12 & $-0.49,0.25$ & 0.524 \\
\hline $\mathrm{BMD}_{\mathrm{TB}}{ }^{\mathrm{h}}$ & -0.17 & $-0.52,0.19$ & 0.357 \\
\hline $\mathrm{BMD}_{\mathrm{LS}}^{\mathrm{i}}$ & -0.08 & $-0.41,0.24$ & 0.607 \\
\hline $\mathrm{BMAD}_{\mathrm{LS}}{ }^{\mathrm{j}}$ & -0.04 & $-0.32,0.41$ & 0.811 \\
\hline
\end{tabular}

${ }^{a}$ confidence interval, b ${ }^{b}$ Compared with healthy controls, cAll NHL survivors $(\mathrm{N}=84)$, d body mass index, e $\mathrm{NHL}$ survivors with a DXA-scan available ( $\mathrm{N}=56$ ), flean body mass, "bone mineral content, "hbone mineral density of the total body, 'bone mineral density of the lumbar spine, ibone mineral apparent density of the lumbar spine. 
Table 3. Determinants of height in NHL survivors.

\begin{tabular}{|c|c|c|c|}
\hline \multirow{2}{*}{$\frac{\text { All survivors }^{\mathrm{a}}}{\text { Variable }}$} & \multicolumn{3}{|c|}{ Height (SDS) } \\
\hline & $\beta$ & P-value & 95\% C.I. \\
\hline Age at follow-up & 0.01 & 0.556 & $-0.02,0.04$ \\
\hline Sex (male) & 0.26 & 0.319 & $-0.25,0.77$ \\
\hline Type of $\mathrm{NHL}^{\mathrm{b}}$ & 0.13 & 0.582 & $-0.33,0.59$ \\
\hline Survivors with a DXA-scan available ${ }^{c}$ & \multicolumn{3}{|c|}{ Height (SDS) } \\
\hline Variable & $\beta$ & P-value & 95\% C.I. \\
\hline Age at follow-up & 0.24 & 0.815 & $-0.03,0.04$ \\
\hline Sex (male) & 0.00 & 0.303 & $-0.23,0.72$ \\
\hline Type of $\mathrm{NHL}^{\mathrm{b}}$ & 0.16 & 0.482 & $-0.30,0.62$ \\
\hline $\mathrm{LBM}^{\mathrm{d}}$ & 0.68 & $<0.001$ & $0.50,0.85$ \\
\hline
\end{tabular}

${ }^{a}$ All NHL survivors ( $\left.\mathrm{N}=84\right)$, ${ }^{b}$ non $B$ versus $B+L C A L, ~ c N H L$ survivors with a DXA-scan available $(\mathrm{N}=56)$, dean body mass.

\section{Body composition}

DXA-scans were performed in 56 survivors (16 females). Sex, age at follow-up, age at diagnosis and type of NHL were not significantly different between survivors with and without a DXAscan available. Percentage fat and BMC were normal in survivors (Table 2). LBM in survivors was significantly lower (mean SDS $-0.47, \mathrm{P}=0.008$ ) compared with controls. LBM is measured in grams and is therefore highly correlated with height $(R=0.76, P<0.001)$. Linear regression analysis with height (SDS) as dependent variable performed in the subgroup of survivors with a DXA-scan available, confirmed this relationship (Table 3). Males and females were not different with respect to percentage fat (mean SDS 0.13 vs. $0.36, P=0.405)$, $\operatorname{LBM}(-0.49$ vs. $-0.41, P=0.839$ ) and $\mathrm{BMC}$ (mean SDS -0.15 vs. $-0.04, \mathrm{P}=0.794$ ). However, compared with healthy controls, $\mathrm{LBM}$ was significantly lower in males (mean SDS -0.49, P=0.008). Non B-NHL and B-NHL survivors did not differ in percentage fat (mean SDS 0.00 vs. $0.23, P=0.448$ ), LBM (mean SDS -0.67 vs. -0.35 , $\mathrm{P}=0.433$ ) and $\mathrm{BMC}$ (mean SDS -0.57 vs. $0.18, P=0.098$ ), but compared with normal controls, non B-NHL survivors had a lower LBM $(P=0.017)$, whereas $B-N H L / L C A L$ survivors did not $(P=0.161)$.

\section{Bone mineral density}

$B M D_{T B}, B M D_{L S}$ and $B M A D_{L S}$ in NHL survivors were not different from healthy controls (Table 2). Males and females were not different with respect to $\mathrm{BMD}_{\text {ТВ }}$ (mean SDS -0.06 vs. $-0.44, P=0.350$ ), $\mathrm{BMD}_{\text {LS }}$ (mean SDS -0.05 vs. $-0.18, \mathrm{P}=0.362$ ) or $\mathrm{BMAD}_{\text {LS }}$ (mean SDS 0.07 vs. $-0.03, \mathrm{P}=0.801$ ). Non $B$ and $B-N H L / L C A L$ survivors were not different with respect to $B M D_{\text {TB }}$ (mean SDS -0.42 vs. $0.04, \mathrm{P}=0.201$ ), $\mathrm{BMD}_{L S}$ (mean SDS -0.18 vs. $-0.00, \mathrm{P}=0.589$ ) or $\mathrm{BMAD}_{L S}$ (mean SDS -0.09 vs. 0.15, $\mathrm{P}=0.504)$. 


\section{Laboratory measurements}

TSH measurements were assessed in 68 survivors and fT4 measurements in 67 survivors. TSH (mean $1.45 \mathrm{mU} / \mathrm{L}, 95 \%$ confidence interval (C.I.) 1.26, 1.64) and fT4 levels (mean $16.5 \mathrm{pmol} / \mathrm{L}$, $95 \%$ C.I. 15.8, 17.1) were within the normal range in all survivors. None of the survivors was treated with thyroid hormones. None of the 71 survivors had IGF-1 SDS lower than -2 or higher than 2, which are considered to be abnormal. However, IGF-1 SDS was significantly lower than 0 in this group of NHL survivors (mean SDS $-0.14, P=0.032$ ). In 20 adult female survivors AMH levels were available (mean $5.3 \mu \mathrm{g} / \mathrm{L}, 95 \%$ C.I. 3.0-7.5). Three survivors had AMH levels lower than $1.0 \mu \mathrm{g} / \mathrm{l}$.

Inhibin B levels were available in 42 out of 43 adult male NHL survivors (mean $149 \mathrm{ng} / \mathrm{L}$, 95\% C.I. 125-173). Twenty-three (55\%) adult male survivors had abnormal (<150 ng/l) Inhibin B levels, of whom 16 (38\%) below $100 \mathrm{ng} / \mathrm{l}$. Additionally, FSH levels were high and correlated with low Inhibin $B$ levels $(R=-0.70, P<0.001)$. In univariate analysis, cumulative dose of administered cytarabine was significantly higher in survivors with low Inhibin $B$ versus survivors with normal Inhibin B. Age at diagnosis and cumulative dose of cyclophosphamide, that have been reported to influence fertility in survivors, were not significantly higher in survivors with low Inhibin B. Because of its known relation with infertility, we also calculated the alkylating agent dose by use of tertiles, as described by Green et al. ${ }^{21,22}$ This enabled us to take into account the effect not only of cyclophosphamide, but also of ifosfamide, busulfan and melphalan, alkylating agents that were administered in our group of NHL survivors. In linear regression analysis, after correction for age at diagnosis, type of NHL and alkylating agent dose, cytarabine had a significant negative effect on Inhibin B levels ( $\beta-8.2, P=0.024$ ) (Table 4). To rule out possible effects of pituitary or hypothalamic damage, two survivors who were treated with total body irradiation (TBI) or craniospinal irradiation were excluded from this analysis.

Table 4. Determinants of Inhibin B levels in adult male NHL survivors.

\begin{tabular}{lccc}
\hline & & Inhibin B & \\
\cline { 2 - 4 } Variable & $\boldsymbol{\beta}$ & P-value & $\mathbf{9 5 \%}$ C.I. \\
\hline Age at diagnosis & 2.9 & 0.484 & $-5.4,11.2$ \\
Type of $\mathrm{NHL}^{\mathrm{a}}$ & -21.8 & 0.567 & $-98.3,54.7$ \\
Alkylating agent dose & -5.3 & 0.805 & $-48.7,38.1$ \\
Cytarabine (gram) & -8.2 & 0.024 & $-15.2,-1.2$ \\
\hline
\end{tabular}

Survivors treated with total body irradiation or craniospinal irradiation are excluded from this analysis. ${ }^{a}$ non $B$ versus $B+L C A L$. 


\section{Discussion}

The present study describes endocrine late sequelae in long-term survivors of childhood NHL. We observed a lower absolute lean body mass (LBM) and a shorter stature in NHL survivors twelve years after cessation of therapy. The lower LBM can be explained by the shorter stature of the survivors, as LBM is described by an absolute weight of tissue not including fat and bone. Lower adult height has been observed in ALL survivors, ${ }^{12,44-47}$ and a correlation has been observed between cranial radiotherapy and reduced height. Some studies observed a correlation between chemotherapy and height, ${ }^{46,47}$ but this is regarded as controversial. We observed height to be lower in NHL survivors twelve years after cessation of therapy, in particular in male survivors. However, as height was already low at diagnosis, it is unlikely that this is due to administered treatment.

Growth hormone deficiency is a frequently occurring phenomenon in survivors of childhood cancer, especially in survivors treated with cranial radiotherapy, ${ }^{25,48}$ most likely caused by direct pituitary damage resulting from cranial irradiation but also by hypothalamic damage. However, growth hormone deficiency has also been described in survivors not treated with radiotherapy. ${ }^{46}$ Growth hormone deficiency can contribute to reduced height, obesity and osteopenia or osteoporosis. Although our survivors had a significantly lower height compared with healthy controls, none of the 71 survivors had IGF-1 SDS lower than -2, which suggests that growth hormone deficiency did not play an important role in our NHL survivors. However, it is noticeable that IGF-1 SDS tended to be low in our group of survivors. Although a low IGF-1 level is a reasonable indicator of growth hormone deficiency, a normal IGF-1 will not rule out growth hormone deficiency. For this, a dynamic assessment of growth hormone is necessary. Since this is a retrospective study we were not able to assess these dynamic tests. It should, moreover, be considered that these tests are more invasive for the survivors, time consuming and therefore expensive.

Adiposity has been frequently reported in leukemia but also in lymphoma survivors. ${ }^{11,30,31}$ In the present study we found normal BMI and normal percentage fat in NHL survivors. Hypothyreoidism, which can contribute to obesity and is reported in survivors, especially after local radiotherapy, ${ }^{49-51}$ did not occur in this group of survivors.

In patients treated for childhood cancer, treatment can have a deleterious effect on the acquisition of peak bone mass, subsequently increasing the risk of fractures at an older age. Reduced BMD has been described in adolescent and adult NHL survivors after stem cell transplantation ${ }^{52}$ and in childhood NHL survivors after chemotherapy combined with cranial radiotherapy. ${ }^{9,29}$ In our study, which is the largest described cohort of long-term childhood NHL survivors so far, $\mathrm{BMD}_{\mathrm{TB}}$ and $B M D_{L S}$ were within the normal range. $B M D$ of the lumbar spine was still normal after correction for bone size $\left(B M A D_{L S}\right)$. This was in concordance with findings in long-term survivors of childhood ALL. ${ }^{9}$ When interpreting these results one should consider the retrospective nature of this study 
and the small sample sizes of the subgroups. However, as the majority of our NHL survivors were treated with chemotherapy only these results seem comprehensible.

Infertility has been frequently reported in childhood cancer survivors. ${ }^{21-23}$ Additionally, low AMH or Inhibin B levels, which are fertility markers, have been reported. ${ }^{24,25}$ Notorious for causing infertility are abdominal radiotherapy and alkylating chemotherapeutic agents, like cyclophosphamide. ${ }^{21,22}$ Moreover, cytarabin has been described to contribute to gonadal damage in survivors of adult acute myeloid leukemia. ${ }^{53}$ However, this was not confirmed in female childhood cancer survivors ${ }^{21}$ and in male childhood cancer survivors even a reversed effect was described. ${ }^{22}$ In the present study, three out of 20 adult female survivors had abnormal AMH levels. Two out of three received both cyclophosphamide and cytarabin, however none of them received cranial or abdominal radiotherapy. Interestingly, Inhibin B levels were low in 55\% of adult male survivors. High FSH levels in these survivors confirm that this is not a problem of pituitary or hypothalamus but of local origin. After correction for age at diagnosis and total cumulative dose of alkylating agents, cytarabin had a significant negative effect on Inhibin B levels in adult males. Although groups are small and results should therefore be interpreted with caution, this effect seems substantial and has not been reported previously. Our results contradict those of Green et al., ${ }^{22}$ which may be due to differences in reported outcomes (siring a pregnancy versus fertility markers). Inhibin B is a relatively new fertility marker but has been shown to be the most reliable serum marker for spermatogenesis. ${ }^{43,54-57}$ Prospective studies are needed to confirm these data, preferably with larger cohorts and including both Inhibin B measurements and sperm analysis. In conclusion, long-term childhood NHL survivors, especially males, have a shorter stature which seems to be determined by height at diagnosis and not by treatment related side effects. Otherwise, this study shows that long term childhood NHL survivors do not seem to be at risk for endocrine late sequelae like osteoporosis, obesity and hypothyreoidy. However, especially males might be at risk for gonadal damage, which may be related to the cumulative dose of cytarabine. To evaluate gonadal damage both in male and female NHL survivors, prospective studies in larger cohorts are required. 


\section{References}

1. Reiter A. Diagnosis and treatment of childhood non-hodgkin lymphoma. Hematology / the Education Program of the American Society of Hematology American Society of Hematology 2007;2007:285-96.

2. Reid IR. Glucocorticoid osteoporosis--mechanisms and management. Eur J Endocrinol 1997;137:20917.

3. van Beek RD, van den Heuvel-Eibrink MM, Hakvoort-Cammel FG, et al. Bone mineral density, growth and thyroid function in long-term survivors of pediatric Hodgkin's lymphoma treated with chemotherapy only. J Clin Endocrinol Metab 2009 Jun;94(6).

4. Arikoski P, Komulainen J, Voutilainen R, et al. Reduced bone mineral density in long-term survivors of childhood acute lymphoblastic leukemia. J Pediatr Hematol Oncol 1998;20:234-40.

5. Brennan BM, Rahim A, Adams JA, Eden OB, Shalet SM. Reduced bone mineral density in young adults following cure of acute lymphoblastic leukemia in childhood. Br J Cancer 1999;79:1859-63.

6. Gilsanz V, Carlson ME, Roe TF, Ortega JA. Osteoporosis after cranial irradiation for acute lymphoblastic leukemia. J Pediatr 1990;117:238-44.

7. Henderson RC, Madsen CD, Davis C, Gold SH. Bone density in survivors of childhood malignancies. J Pediatr Hematol Oncol 1996;18:367-71.

8. van der Sluis IM, van den Heuvel-Eibrink MM, Hahlen K, Krenning EP, de Muinck Keizer-Schrama SM. Bone mineral density, body composition, and height in long-term survivors of acute lymphoblastic leukemia in childhood. Med Pediatr Oncol 2000;35:415-20.

9. Nysom K, Holm K, Hertz H, Muller J, Michaelsen KF, Molgaard C. Bone mass after treatment for acute Iymphoblastic leukemia in childhood. J Clin Oncol 2001;19:2970-1.

10. van Beek RD, de Muinck Keizer-Schrama SM, Hakvoort-Cammel FG, et al. No difference between prednisolone and dexamethasone treatment in bone mineral density and growth in long term survivors of childhood acute lymphoblastic leukemia. Pediatr Blood Cancer 2006;46:88-93.

11. Asner S, Ammann RA, Ozsahin H, Beck-Popovic M, von der Weid NX. Obesity in long-term survivors of childhood acute lymphoblastic leukemia. Pediatr Blood Cancerr 2008;51:118-22.

12. Birkebaek NH, Clausen N. Height and weight pattern up to 20 years after treatment for acute lymphoblastic leukemia. Arch Dis Child 1998;79:161-4.

13. Jarfelt M, Lannering B, Bosaeus I, Johannsson G, Bjarnason R. Body composition in young adult survivors of childhood acute lymphoblastic leukemia. Eur J Endocrinol 2005;153:81-9.

14. Oeffinger KC, Buchanan GR, Eshelman DA, et al. Cardiovascular risk factors in young adult survivors of childhood acute lymphoblastic leukemia. J Pediatr Hematol Oncol 2001;23:424-30.

15. Warner JT, Evans WD, Webb DK, Gregory JW. Body composition of long-term survivors of acute lymphoblastic leukemia. Med Pediatr Oncol 2002;38:165-72.

16. Brennan BM, Rahim A, Blum WF, Adams JA, Eden OB, Shalet SM. Hyperleptinemia in young adults following cranial irradiation in childhood: growth hormone deficiency or leptin insensitivity? Clin Endocrinol 1999;50:163-9.

17. Nysom K, Holm K, Michaelsen KF, Hertz H, Muller J, Molgaard C. Degree of fatness after treatment for acute lymphoblastic leukemia in childhood. J Clin Endocrinol Metab 1999;84:4591-6.

18. van Waas M, Neggers SJ, Pieters R, van den Heuvel-Eibrink MM. Components of the metabolic syndrome in 500 adult long-term survivors of childhood cancer. Ann Oncol 2010;21:1121-6.

19. van Waas M, Neggers SJ, van der Lelij AJ, Pieters R, van den Heuvel-Eibrink MM. The metabolic syndrome in adult survivors of childhood cancer, a review. J Pediatr Hematol Oncol 2010;32:171-9.

20. Steffens M, Beauloye V, Brichard B, et al. Endocrine and metabolic disorders in young adult survivors of childhood acute lymphoblastic leukemia (ALL) or non-Hodgkin lymphoma (NHL). Clin Endocrinol 2008;69:819-27.

21. Green DM, Kawashima T, Stovall M, et al. Fertility of female survivors of childhood cancer: a report from the childhood cancer survivor study. J Clin Oncol 2009;27:2677-85. 
22. Green DM, Kawashima T, Stovall M, et al. Fertility of male survivors of childhood cancer: a report from the Childhood Cancer Survivor Study. J Clin Oncol 2010;28:332-9.

23. Hudson MM. Reproductive outcomes for survivors of childhood cancer. Obstet Gynecol 2010;116:117183.

24. Lie Fong S, Laven JS, Hakvoort-Cammel FG, et al. Assessment of ovarian reserve in adult childhood cancer survivors using anti-Mullerian hormone. Human Reprod (Oxford, England) 2009;24:982-90.

25. van Casteren NJ, van der Linden GH, Hakvoort-Cammel FG, Hahlen K, Dohle GR, van den Heuvel-Eibrink MM. Effect of childhood cancer treatment on fertility markers in adult male long-term survivors. Pediatr Blood Cancer 2009;52:108-12.

26. Nysom K, Holm K, Michaelsen KF, Hertz H, Muller J, Molgaard C. Degree of fatness after treatment of malignant lymphoma in childhood. Med Pediatr Oncol 2003;40:239-43.

27. Muszynska-Roslan K, Konstantynowicz J, Krawczuk-Rybak M, Protas P. Body composition and bone mass in survivors of childhood cancer. Pediatr Blood Cancer 2007;48:200-4.

28. Vassilopoulou-Sellin R, Brosnan P, Delpassand A, Zietz H, Klein MJ, Jaffe N. Osteopenia in young adult survivors of childhood cancer. Med Pediatr Oncol 1999;32:272-8.

29. Sala A, Talsma D, Webber C, Posgate S, Atkinson S, Barr R. Bone mineral status after treatment of malignant lymphoma in childhood and adolescence. Eur J Cancer 2007;16:373-9.

30. Gofman I, Ducore J. Risk factors for the development of obesity in children surviving ALL and NHL. J Pediatr Hematol Oncol 2009;31:101-7.

31. Razzouk $\mathrm{BI}$, Rose SR, Hongeng $\mathrm{S}$, et al. Obesity in survivors of childhood acute lymphoblastic leukemia and lymphoma. J Clin Oncol 2007;25:1183-9.

32. Assembly WMAG. Declaration of Helsinki - Sixth revision. 2008.

33. Fredriks AM, van Buuren S, Burgmeijer RJ, et al. Continuing positive secular growth change in The Netherlands 1955-1997. Pediatr Res 2000;47:316-23.

34. Fredriks AM, van Buuren S, Jeurissen SE, Dekker FW, Verloove-Vanhorick SP, Wit JM. Height, weight, body mass index and pubertal development reference values for children of Turkish origin in the Netherlands. Eur J Pediatr 2003;162:788-93.

35. Fredriks AM, van Buuren S, Jeurissen SE, Dekker FW, Verloove-Vanhorick SP, Wit JM. Height, weight, body mass index and pubertal development references for children of Moroccan origin in The Netherlands. Acta Paediatr 2004;93:817-24.

36. A. Blokstra HAS, H.B. Bueno de Mesquita, and W.M.M.V. J.C. Seidell. Monitoring van Risicofactoren en Gezondheid in Nederland (MORGEN-project), 1993-1997. 1999.

37. Kroger H, Vainio P, Nieminen J, Kotaniemi A. Comparison of different models for interpreting bone mineral density measurements using DXA and MRI technology. Bone 1995;17:157-9.

38. van der Sluis IM, de Ridder MA, Boot AM, Krenning EP, de Muinck Keizer-Schrama SM. Reference data for bone density and body composition measured with dual energy $x$ ray absorptiometry in white children and young adults. Arch Dis Child 2002;87:341-7; discussion -7.

39. Elmlinger MW, Kuhnel W, Weber MM, Ranke MB. Reference ranges for two automated chemiluminescent assays for serum insulin-like growth factor I (IGF-I) and IGF-binding protein 3 (IGFBP-3). Clin Chem Lab Med 2004;42:654-64.

40. de Vet A, Laven JS, de Jong FH, Themmen AP, Fauser BC. Antimullerian hormone serum levels: a putative marker for ovarian aging. Fertil Steril 2002;77:357-62.

41. Kevenaar ME, Meerasahib MF, Kramer P, et al. Serum anti-mullerian hormone levels reflect the size of the primordial follicle pool in mice. Endocrinology 2006;147:3228-34.

42. van Casteren NJ, Dohle GR, Romijn JC, de Muinck Keizer-Schrama SM, Weber RF, van den HeuvelEibrink MM. Semen cryopreservation in pubertal boys before gonadotoxic treatment and the role of endocrinologic evaluation in predicting sperm yield. Fertil Steril 2008;90:1119-25.

43. Pierik FH, Burdorf A, de Jong FH, Weber RF. Inhibin B: a novel marker of spermatogenesis. Ann Med 2003;35:12-20.

44. Schriock EA, Schell MJ, Carter M, Hustu O, Ochs JJ. Abnormal growth patterns and adult short stature in 115 long-term survivors of childhood leukemia. J Clin Oncol 1991;9:400-5. 
45. Katz JA, Pollock BH, Jacaruso D, Morad A. Final attained height in patients successfully treated for childhood acute lymphoblastic leukemia. J Pediatr 1993;123:546-52.

46. Sklar C, Mertens A, Walter A, et al. Final height after treatment for childhood acute lymphoblastic leukemia: comparison of no cranial irradiation with 1800 and 2400 centigrays of cranial irradiation. J Pediatr 1993;123:59-64.

47. Dalton VK, Rue M, Silverman LB, et al. Height and weight in children treated for acute lymphoblastic leukemia: relationship to CNS treatment. J Clin Oncol 2003;21:2953-60.

48. Mulder RL, Kremer LC, van Santen HM, et al. Prevalence and risk factors of radiation-induced growth hormone deficiency in childhood cancer survivors: a systematic review. Cancer Treat Rev 2009;35:61632.

49. Bonato $\mathrm{C}$, Severino RF, Elnecave RH. Reduced thyroid volume and hypothyroidism in survivors of childhood cancer treated with radiotherapy. J Pediatr Endocrinol Metab 2008;21:943-9.

50. Miyoshi Y, Ohta H, Hashii Y, et al. Endocrinological analysis of 122 Japanese childhood cancer survivors in a single hospital. Endocr J 2008;55:1055-63.

51. Stava CJ, Jimenez C, Vassilopoulou-Sellin R. Endocrine sequelae of cancer and cancer treatments. J Cancer Surviv 2007;1:261-74.

52. Majhail NS, Ness KK, Burns LJ, et al. Late effects in survivors of Hodgkin and non-Hodgkin lymphoma treated with autologous hematopoietic cell transplantation: a report from the bone marrow transplant survivor study. Biol Blood Marrow Transplant 2007;13:1153-9.

53. Lemez P, Urbanek V. Chemotherapy for acute myeloid leukemias with cytosine arabinoside, daunorubicin, etoposide, and mitoxantrone may cause permanent oligoasthenozoospermia or amenorrhea in middle-aged patients. Neoplasma 2005;52:398-401.

54. Pierik FH, Vreeburg JT, Stijnen T, De Jong FH, Weber RF. Serum inhibin B as a marker of spermatogenesis. $\mathrm{J}$ Clin Endocrinol Metab 1998;83:3110-4.

55. Kumanov P, Nandipati K, Tomova A, Agarwal A. Inhibin B is a better marker of spermatogenesis than other hormones in the evaluation of male factor infertility. Fertil Steril 2006;86:332-8.

56. Klingmuller D, Haidl G. Inhibin B in men with normal and disturbed spermatogenesis. Human Reprod (Oxford, England) 1997;12:2376-8.

57. van Beek RD, Smit M, van den Heuvel-Eibrink MM, et al. Inhibin B is superior to FSH as a serum marker for spermatogenesis in men treated for Hodgkin's lymphoma with chemotherapy during childhood. Human Reprod (Oxford, England) 2007;22:3215-22. 


\section{Abstract}

Background: Adrenal insufficiency, or relative insufficiency, might partly explain increased mortality rates in nephroblastoma and neuroblastoma survivors after unilateral adrenalectomy. Aim was to assess adrenal function and its metabolic effects in survivors after adrenalectomy.

Patients and methods: In this prospective study, 67 adult long-term survivors of nephroblastoma and 36 survivors of neuroblastoma participated. Forty-nine control subjects were crosssectionally recruited. Adrenal function was assessed by a $1 \mu \mathrm{g}$ short Synacthen-test. Levels of cortisol, adrenocorticotrophic hormone (ACTH), low (LDL-C) and high-density lipoproteincholesterol (HDL-C), triglycerides, apolipoprotein-B, glucose and insulin were assessed in blood samples taken at baseline. In addition, cortisol levels were assessed after $30(t=30)$ and 60 minutes. Homeostatic Model Assessment (HOMA) was calculated.

Results: Adrenal insufficiency was not present in survivors. Interestingly, baseline serum cortisol levels were higher in survivors after unilateral adrenalectomy (mean $503 \mathrm{nmol} / \mathrm{l})(\mathrm{N}=46)$ than in survivors with both adrenals intact (mean $393 \mathrm{nmol} / \mathrm{l}, \mathrm{P}=0.002)(\mathrm{N}=52)$, and than in controls (mean $399 \mathrm{nmol} / \mathrm{l}, \mathrm{P}=0.013$ ) ( $\mathrm{N}=49)$. After correcting for age, sex, and use of oral estrogens, unilateral adrenalectomy was independently associated with elevated baseline cortisol and ACTH levels. Baseline cortisol levels were positively associated with triglycerides $(P<0.001)$, LDL-C ( $P=0.004)$, apolipoprotein-B $(P<0.001)$ and HOMA $(P=0.008)$.

Conclusions: No adrenal insufficiency was observed in survivors of nephroblastoma and neuroblastoma. Survivors treated with unilateral adrenalectomy had relatively high basal cortisol and ACTH levels, indicating a higher central setpoint of the hypothalamic-pituitary-adrenal axis. This higher setpoint was associated with lipid concentrations and insulin resistance and can therefore influence the cardiovascular risk profile in long term survivors of nephroblastoma and neuroblastoma. 


\section{Introduction}

Over the past decades, childhood cancer survival rates have increased significantly. Approximately $75 \%$ of children diagnosed with cancer are cured and become long-term survivors. For children diagnosed with nephroblastoma or neuroblastoma 5 -year survival is approximately $92 \%$ and $55 \%$, respectively. Increased long-term survival has led to increasing absolute numbers of survivors: to date 1 in 640 young adults is a childhood cancer survivor. ${ }^{1}$ Therefore, the recognition of late effects has become more relevant. In nephroblastoma and neuroblastoma survivors, especially those treated with radiotherapy, the risk for developing late adverse events like second malignant neoplasms and pulmonary, orthopaedic and cardiovascular events, muscoskeletal late effects, cardiac toxicity, reproductive health problems and renal dysfunction is significant. ${ }^{2-4}$ Due to poor survival, reports on late effects in intensively treated long-term neuroblastoma survivors are mainly based on case series and small cohorts.

Although endocrine sequelae are the most common late effects, ${ }^{5-15}$ little is known about the long-term endocrine effects in survivors of nephroblastoma or neuroblastoma. Increased mortality rates as compared to the general age-matched population have been reported in childhood cancer survivors, which are partly related to the above-mentioned documented late effects. ${ }^{16}$ However unexpected deaths occur and to date it is unknown whether adrenal insufficiency following surgery, radiotherapy and/or chemotherapy for nephroblastoma or neuroblastoma is an important denominator of this excess mortality rate later in life. Abdominal surgery is part of treatment for nephroblastoma and neuroblastoma. In the majority of nephroblastoma patients, unilateral total nephrectomy, often including unilateral adrenalectomy, is performed. In neuroblastoma patients, adrenalectomy is performed when the tumor is located in the adrenal gland. Bilateral adrenalectomy is associated with high morbidity and mortality rates as a consequence of adrenal insufficiency. ${ }^{17,18} \mathrm{It}$ is unknown whether adrenal function after unilateral adrenalectomy declines over time by aging, especially in survivors who were also treated with chemotherapy or local radiotherapy. We hypothesized that a subset of neuroblastoma and nephroblastoma survivors has a subnormal adrenal function and therefore performed a study on the influence of administered treatment on adrenal function in long term nephroblastoma and neuroblastoma survivors. Additionally, to evaluate the possible altered effect of the hypothalamic-pituitary-adrenal (HPA) axis on the metabolic status in these survivors, we assessed the influence of cortisol on lipids and insulin resistance parameters.

\section{Methods}

\section{Patients}

All long-term ( $\geq 5$ years after cessation of treatment) adult survivors of childhood nephroblastoma and neuroblastoma, treated between 1961 - 2004 in the Erasmus MC-Sophia Children's Hospital 
that regularly visit the Late Effects outpatient clinic were invited to participate in this prospective study. Informed consent was obtained according to the Helsinki declaration ${ }^{19}$ and the study was approved by the local medical ethical committee.

Of the 88 adult long-term survivors of nephroblastoma who were alive and currently living in The Netherlands, 67 participated, six were lost to follow-up, 13 refused to participate and two survivors were not able to visit the outpatient clinic at the appointed time interval. Adult survivors with neuroblastoma stage $4 \mathrm{~s}$ who did not receive surgery, radiotherapy or chemotherapy were excluded from this study. Of the 50 eligible neuroblastoma survivors, 36 survivors participated, five were lost to follow-up, six refused to participate and three females were pregnant at the time of the study. Information on disease and treatment was obtained from our local database and medical records. Data regarding (partial) unilateral adrenalectomy were obtained from pathology reports. As to date limited normal values for young adults are available, we recruited a control group, consisting of siblings, friends or neighbours, preferably of the same sex and within an age range of five years of their related survivor. This group was designed as a socio-demographically similar comparison population. Information regarding smoking status and socio-economic status (defined by the highest level of educational attainment) was collected using a questionnaire. Height was measured to the nearest millimeter using a Harpenden Stadiometer and weight was measured without shoes and clothes but in underwear to the nearest $0.1 \mathrm{~kg}$ with a standard clinical balance. Body mass index (BMI) was calculated as weight in kilograms divided by the squared length in meters. Survivors and controls participated in this study from October 2009 until March 2011.

\section{ACTH stimulation test}

To evaluate adrenal function, a low dose $(1 \mu \mathrm{g})$ short ACTH stimulation-test was performed both in survivors and control subjects. ACTH stimulation-tests were started in the morning, all before 10:00 am. Subjects were resting during one hour and during the test. Fasting blood samples were taken from one and the same intravenous-cannula at baseline, after 30 minutes ( $t=30)$ and after 60 minutes $(t=60)$. One milliliter of Synacthen solution $250 \mu \mathrm{g} / \mathrm{ml}$ (Defiante Farmaceutica S.A., Portugal) was added to $249 \mathrm{ml}$ of sterile solution of $\mathrm{NaCl} 0.9 \%$. One milliliter of this solution, corresponding to $1 \mu \mathrm{g}$ of Synacthen, was administered intravenously after the baseline blood sample was taken. Serum cortisol levels were measured at baseline, $t=30$ and $t=60$. At $t=30$, cortisol levels higher than $440 \mathrm{nmol} / \mathrm{I}$ were considered normal, adrenal insufficiency was defined as cortisol levels below $440 \mathrm{nmol} / \mathrm{l}$ at $\mathrm{t}=30 .{ }^{20} \mathrm{In}$ addition, ACTH was measured in the sample taken at baseline.

\section{Laboratory measurements}

Serum cortisol (nmol/l), ACTH (pmol/l), androstenedione (adione) (nmol/l), dehydroepiandosterone-sulfate (DHEAs) ( $\mu \mathrm{mol} / \mathrm{l})$, insulin $(\mathrm{p} / \mathrm{mol} / \mathrm{l})$, testosterone $(\mathrm{nmol} / \mathrm{l})$, dehydroepiandosterone (DHEA) (nmol/l), 17-hydroxyprogesterone (17OHP) (nmol/l), triglycerides, high- 
density lipoprotein-cholesterol (HDL-C), low-density lipoprotein-cholesterol (LDL-C), glucose and apolipoprotein-B (Apo-B) (g/I) were measured. Assays and intra- and inter coefficients of variation are described in supplementary text. Homeostatic Model Assessment (HOMA) was calculated as a measure of insulin resistance..$^{21}$

\section{Statistics}

Statistical analysis was performed with the Statistical Package for Social Sciences (SPSS 17.0, Chicago, IL, USA). Data are expressed as means and standard deviations, unless specified otherwise. Independent Sample T-tests were used to compare results in survivors and controls and in subgroups. The associations between cortisol levels and various treatment parameters and baseline characteristics were analyzed using multiple linear regression modelling. Cortisol, ACTH, triglycerides and glucose levels were normally distributed after log-transformation. Beta's of logtransformed variables are expressed in percentages. Age and sex were added to all models and influence of BMI was evaluated as previously recommended. ${ }^{22}$ Estrogens, as the component of hormonal contraception or as oral supplementals in estrogen deficient women, are known to increase serum levels of cortisol-binding globulin (CBG) resulting in higher total serum cortisol levels. Accordingly, serum cortisol concentrations do not correlate well with cortisol production rates unless the cortisol binding globulin concentration is accounted for. ${ }^{23}$ Twenty-nine out of 73 females ( $9 / 25$ controls, $20 / 48$ survivors) in our study used oral estrogens. Therefore, oral estrogen use was added to the model. Furthermore, three subjects were using fluticasone (one survivor with both adrenals intact, one survivor who underwent adrenalectomy, one control subject) and one survivor who underwent adrenalectomy was using hydrocortisone cream. A subanalysis was performed excluding these survivors. In the first model we added a dummy variable for patients with intact adrenals and a dummy variable for patients who had undergone an adrenalectomy. In the second model, we added cumulative dose of anthracyclines, vincristine, actinomycine, being treated with cyclophosphamide or not (dichotomous variable) and radiotherapy administered to the abdomen or to the contralateral adrenal gland. The variables smoking (smoker, non-smoker or former smoker) and socio-economic status (ordinal variable) were added to both models, but these factors were not significant. Additionally, multivariate analyses were performed to assess the influence of cortisol levels on metabolic parameters. Models were corrected for age, sex, socio-economic status, BMI, diagnosis, abdominal radiotherapy and cumulative dose of administered chemotherapy. P-values $<0.05$ (two-tailed) were considered statistically significant.

\section{Results}

\section{Survivors and controls}

Sixty-seven long-term adult nephroblastoma survivors (28 females) and 36 neuroblastoma survivors (21 females) participated in this study. Forty-eight percent of survivors had a sibling 
or friend willing to participate, resulting in 49 participating control subjects ( 23 females). Thirty-six of them were siblings and 13 were partner or friend of the survivors. The main reason for survivors not to bring a control-subject was the inability or unwillingness of siblings or neighbours to participate as they had to take a day off at work. Baseline and treatment characteristics and status of the adrenal glands are shown in Table 1. In survivors who underwent adrenalectomy the tumor was located in or close to the adrenal gland, in survivors treated with partial adrenalectomy the tumor was located at the upper part of the kidney and in survivors treated without adrenalectomy the tumor was located at the lower part of the kidney. Synacthen stimulation-tests were performed in 93/103 survivors and in 48/49 controls and baseline cortisol levels were available in 101/103 survivors and in all controls. In 3/103 survivors information regarding adrenalectomy was not available, they were therefore excluded in the relevant analysis.

\section{Baseline levels}

Mean baseline cortisol level in nephroblastoma and neuroblastoma survivors was $450 \mathrm{nmol} / \mathrm{l}$ and in controls $399 \mathrm{nmol} / \mathrm{I}(\mathrm{P}=0.141$ ). Two survivors (1 nephroblastoma and 1 neuroblastoma) and two control subjects had cortisol levels higher than $1000 \mathrm{nmol} / \mathrm{l}$ at baseline. Baseline cortisol levels were not different between nephroblastoma or neuroblastoma survivors (mean 461 vs. mean $429, \mathrm{P}=0.44$, Supplementary table 1). Interestingly, baseline serum cortisol levels were higher in survivors after unilateral adrenalectomy compared with survivors without adrenalectomy $(P=0.002)$, and also higher when compared with controls ( $P=0.013)$ (Figure 1a). The percentage of estrogen users was not significantly different between the two survivor subgroups $(P=0.203)$. After correcting for confounders the effect of adrenalectomy remained significant $(P=0.010)$ in the total group (Table 2 ), in the subanalysis excluding females on oral estrogens $(\beta=28.3, P=0.002$ ) and in the subanalysis excluding survivors who were currently using corticosteroids $(\beta=20.0$, $P=0.019)$. Cumulative dose of anthracyclines $(\beta=-0.0002, P=0.995)$, vincristine $(\beta=-0.4, P=0.121)$ and actinomycin $(\beta=0.8, P=0.297)$, being treated with cyclophosphamide $(\beta=-3.3, P=0.723)$, abdominal radiotherapy $(\beta=-2.3, P=0.789)$ and radiotherapy administered to the contralateral adrenal gland $(\beta=17.2, P=0.244)$ were not significant.

Mean ACTH levels were $5.3 \mathrm{pmol} / \mathrm{l}$ both in controls and in survivors with both adrenals intact, and were $7.8 \mathrm{pmol} / \mathrm{l}$ in survivors after adrenalectomy. After correcting for age, sex, oral estrogen use and BMI, adrenalectomy was positively associated with baseline ACTH levels both in the total group ( $\beta=49.2, P=0.006)$, in the subanalysis excluding females on oral estrogens $(\beta=39.5, P=0.036)$ and in the subanalysis excluding survivors who were currently using corticosteroids $(\beta=46.4$, $\mathrm{P}=0.012$ ). Adding the cumulative dose of various chemotherapeutic agents and radiotherapy to the model did not affect the results (data not shown). 
Table 1. Baseline characteristics of study participants.

\begin{tabular}{|c|c|c|c|c|c|}
\hline & \multicolumn{2}{|r|}{ Nephroblastoma } & \multicolumn{2}{|r|}{ Neuroblastoma } & Controls \\
\hline $\mathrm{N}$ & \multicolumn{2}{|r|}{67} & \multicolumn{2}{|r|}{36} & 49 \\
\hline Male/female & \multicolumn{2}{|r|}{$39 / 28$} & & $15 / 21$ & $26 / 23$ \\
\hline Age at follow-up (yrs) & \multicolumn{2}{|r|}{$30.2(18.8-50.8)$} & & $29.6(20.4-46.2)$ & $32.7(18.0-61.8)$ \\
\hline Age at diagnosis (yrs) & \multicolumn{2}{|r|}{$3.3(0.0-12.7)$} & & $0.8(0.0-11.7)$ & n.a. \\
\hline Follow-up time $(y r s)^{a}$ & \multicolumn{2}{|r|}{$26.2(6.4-48.9)$} & \multicolumn{2}{|r|}{$27.8(15.0-44.4)$} & n.a. \\
\hline \multicolumn{6}{|l|}{ Stage } \\
\hline 1 & \multicolumn{2}{|r|}{32} & & 6 & \multirow[t]{6}{*}{ n.a. } \\
\hline 2 & \multicolumn{2}{|r|}{13} & \multicolumn{2}{|r|}{17} & \\
\hline 3 & \multicolumn{2}{|r|}{11} & \multicolumn{2}{|r|}{8} & \\
\hline 4 & \multicolumn{2}{|r|}{3} & \multicolumn{2}{|r|}{2} & \\
\hline 5 & \multicolumn{2}{|r|}{1} & \multicolumn{2}{|r|}{0} & \\
\hline unknown & \multicolumn{2}{|r|}{7} & \multicolumn{2}{|r|}{3} & \\
\hline Surgery (n) & \multicolumn{2}{|r|}{67} & \multicolumn{2}{|r|}{36} & n.a. \\
\hline unilateral adrenalectomy & \multicolumn{2}{|r|}{26} & \multicolumn{2}{|r|}{13} & n.a. \\
\hline partial adrenalectomy & \multicolumn{2}{|r|}{7} & \multicolumn{2}{|r|}{0} & \\
\hline no adrenalectomy & & 31 & & 23 & \\
\hline unknown & & 3 & & 0 & \\
\hline Radiotherapy (n) & & 35 & & 12 & n.a. \\
\hline hemi-abdomen & & 20 & & 0 & n.a. \\
\hline abdomen & & 5 & & 7 & \\
\hline kidney & & 7 & & 0 & \\
\hline lung(s) & & 3 & & 0 & \\
\hline thorax & & 0 & & 2 & \\
\hline spine & & 0 & & 1 & \\
\hline neck & & 0 & & 1 & \\
\hline Cumulative dose (Gy) & & $25(15-40)$ & & $20(10-30)$ & n.a. \\
\hline Chemotherapy (n) & & 59 & & 31 & n.a. \\
\hline & $\mathbf{N}$ & Cumulative dose & $\mathbf{N}$ & Cumulative dose & \\
\hline Vincristin $\left(\mathrm{mg} / \mathrm{m}^{2}\right)$ & 51 & $22.0(6.0-93.0)$ & 16 & $22.8(5.0-65.0)$ & n.a. \\
\hline Actinomycin $\mathrm{D}\left(\mathrm{mg} / \mathrm{m}^{2}\right)$ & 48 & $10.9(0.1-24.8)$ & 0 & 0 & n.a. \\
\hline Anthracyclines $\left(\mathrm{mg} / \mathrm{m}^{2}\right)$ & 18 & $250(100-450)$ & 12 & $210(100-315)$ & n.a. \\
\hline Cyclophosphamide $\left(\mathrm{mg} / \mathrm{m}^{2}\right)$ & 2 & $3825(250-7400)$ & 29 & 7350 (3150 - 45990) & n.a. \\
\hline Cisplatin $\left(\mathrm{mg} / \mathrm{m}^{2}\right)$ & 0 & 0 & 6 & $450(270-630)$ & n.a. \\
\hline Teniposide $\left(\mathrm{mg} / \mathrm{m}^{2}\right)$ & 0 & 0 & 6 & $500(300-700)$ & n.a. \\
\hline Dacarbazine $\left(\mathrm{mg} / \mathrm{m}^{2}\right)$ & 2 & $14.7(13.5-15.8)$ & 0 & 0 & n.a. \\
\hline Iphosphamide $\left(\mathrm{mg} / \mathrm{m}^{2}\right)$ & 2 & $33000(30000-36000)$ & 0 & 0 & n.a. \\
\hline
\end{tabular}

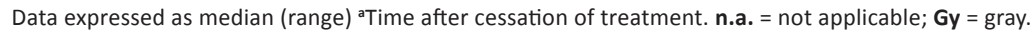




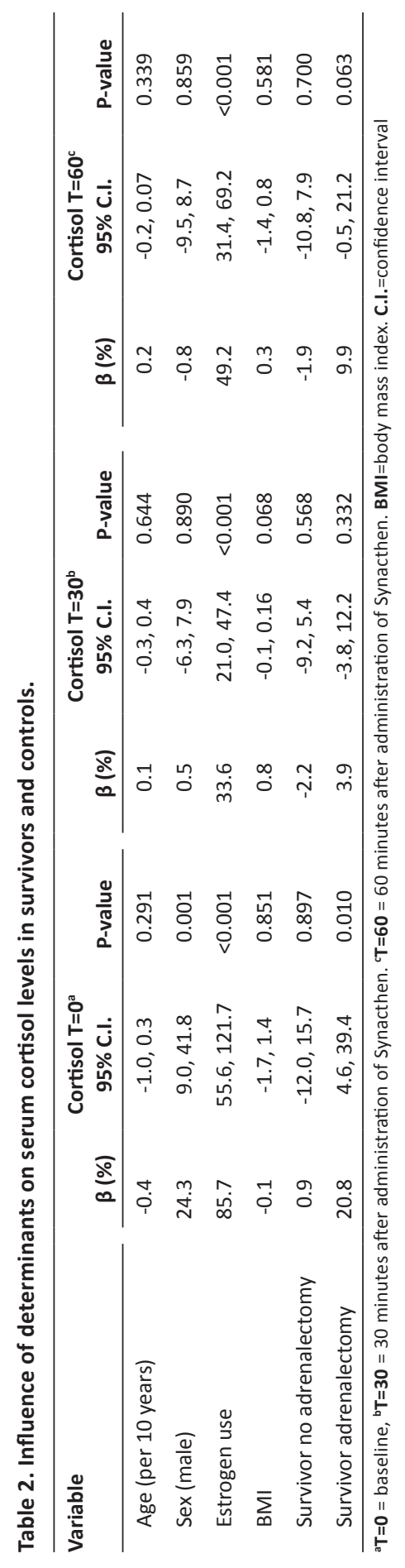




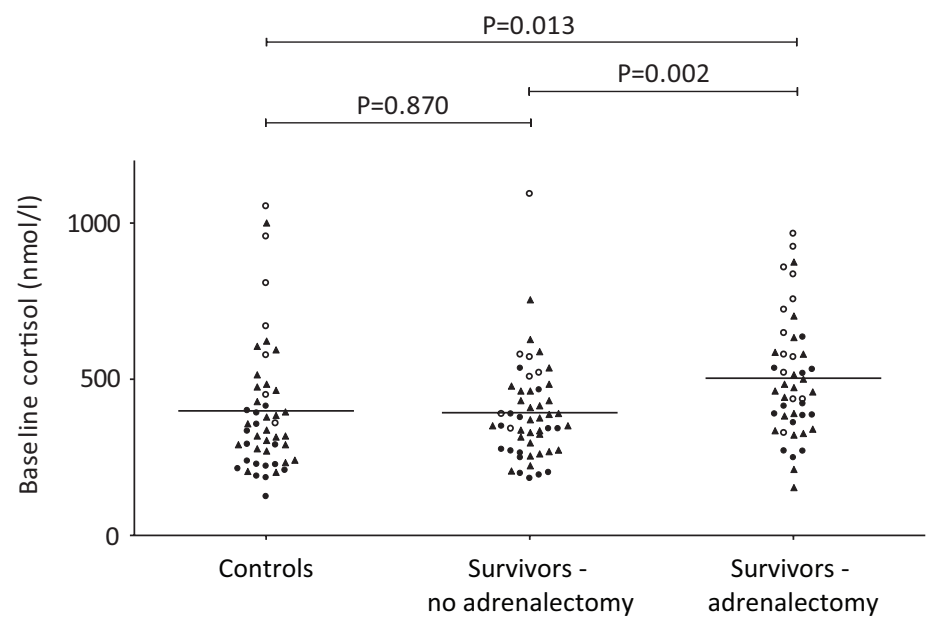

Figure 1a. Baseline cortisol levels.

\section{Normal controls}

To confirm that $440 \mathrm{nmol} / \mathrm{l}$ is a correct cut-off value for serum cortisol at $\mathrm{t}=30$ in subjects of this age group, 47 control subjects were evaluated. Mean cortisol level at $t=30$ in our controls was $662 \mathrm{nmol} / \mathrm{l}$ (range 417 - 1200). Ninety-six percent $(\mathrm{N}=45)$ of controls had serum cortisol levels higher than $440 \mathrm{nmol} / \mathrm{l}, 4 \%(\mathrm{~N}=2)$ of controls had levels below $440 \mathrm{nmol} / \mathrm{l}(419 \mathrm{nmol} / \mathrm{l}$ and $430 \mathrm{nmol} / \mathrm{l})$. These subjects were further investigated using an insulin tolerance test and then reached a peak cortisol level of $>550 \mathrm{nmol} / \mathrm{l}$, indicating that adrenal function in these subjects was sufficient. Fifteen percent of controls had cortisol levels at $t=30$ below $500 \mathrm{nmol} / \mathrm{l}$, versus $5 \%$ of survivors.

\section{Adrenal insufficiency}

In order to evaluate adrenal insufficiency, cortisol levels at $\mathrm{t}=30$ after administration of Synacthen were analyzed. None of the nephroblastoma or neuroblastoma survivors had cortisol levels below $440 \mathrm{nmol} / \mathrm{l}$ at $\mathrm{t}=30$ (Figure $1 \mathrm{~b}$ ). Serum cortisol levels at $\mathrm{t}=30$ were not different between long-term survivors of nephroblastoma, neuroblastoma or controls. However, serum cortisol levels at $\mathrm{t}=30$ and $\mathrm{t}=60$ were significantly higher in survivors after adrenalectomy compared with survivors with both adrenals intact (Appendix table 1 - 2). Concordantly, mean increase of cortisol level after Synacthen administration was lower in survivors after adrenalectomy ( $210 \mathrm{nmol} / \mathrm{l}$ ) compared with both controls (mean $266 \mathrm{nmol} / \mathrm{l}, \mathrm{P}=0.034$ ) and survivors with both adrenals intact (mean $262 \mathrm{nmol} / \mathrm{l}, \mathrm{P}=0.032$ ). 


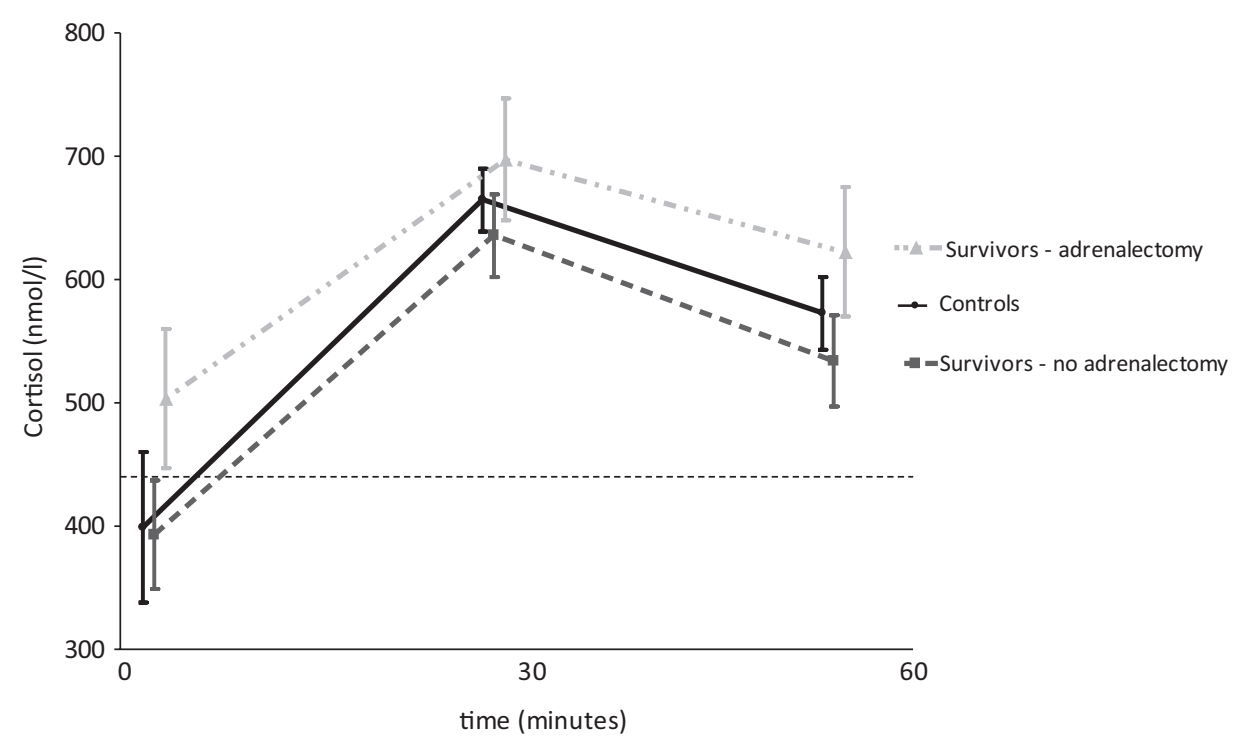

Figure 1b. Synacthen-test.

Data shown as mean ( $95 \%$ confidence interval). To avoid overlapping spaces are introduced on the $x$-axis between subgroups.

\section{Other adrenal steroids}

DHEA, DHEAs, adione and 17-OHP were measured both in males and females, testosterone was only measured in females, all at baseline, and after 30 minutes and 60 minutes. All steroids in males followed a pattern similar to that of cortisol. Steroids in females (estrogen users and non-estrogen users) followed a somewhat less clear pattern. For most steroids, baseline levels in adrenalectomized patients were higher than in non-adrenalectomized patients. However, no significance was reached (Appendix tables $3-4$ ).

\section{Influence of baseline cortisol levels on metabolic parameters}

Baseline cortisol levels were positively associated with triglyceride $(P<0.001), L D L-C(P=0.004)$ and Apo-B $(P<0.001)$ levels, but not with insulin or glucose levels. However, association of baseline cortisol levels with HOMA was significant $(P=0.008)$ (Table 3$)$. 
Table 3. Influence of baseline cortisol levels on metabolic parameters.

\begin{tabular}{|c|c|c|c|}
\hline & \multicolumn{3}{|c|}{ Cortisol (per $100 \mathrm{mmol} / \mathrm{I})$} \\
\hline & $\beta$ & $95 \% \mathrm{Cl}$ & P-value \\
\hline Glucose (\%) ${ }^{\mathrm{a}}$ & 0.90 & $-0.00,0.02$ & 0.138 \\
\hline Insulin (pmol/l) & 1.86 & $-4.52,8.23$ & 0.570 \\
\hline $\mathrm{HOMA}^{\mathrm{a}}$ & 0.06 & $0.02,0.10$ & 0.008 \\
\hline Triglycerides (\%) & 10.52 & $6.61,14.45$ & $<0.001$ \\
\hline HDL-C (mmol/l) & -0.02 & $-0.05,0.00$ & 0.105 \\
\hline LDL-C (mmol/l) & 0.09 & $0.03,0.15$ & 0.004 \\
\hline Apo-B (g/l) & 0.04 & $0.02,0.06$ & $<0.001$ \\
\hline
\end{tabular}

Corrected for: age, sex, socio-economic status, smoking status, diagnosis, body mass index, abdominal radiotherapy, cumulative dose of anthracyclines, vincristin, actinomycin and cyclophosphamide. HOMA = Homeostatic Model Assessment. ${ }^{a}$ subjects with diabetes excluded

\section{Discussion}

The present study describes adrenal function in very long-term (mean 27 years) adult survivors of childhood nephroblastoma and neuroblastoma, in a substantial subset of whom unilateral adrenalectomy was performed. To our knowledge, this is the first study in such a relatively large cohort of nephroblastoma and neuroblastoma survivors. We observed that all nephroblastoma and neuroblastoma survivors, regardless of the presence of both or one adrenal gland(s), had adequate adrenal function. It is therefore unlikely that adrenal dysfunction contributes to the unexplained mortality rate in childhood cancer survivors.

However, we unexpectedly found baseline cortisol and ACTH levels to be higher in survivors treated with adrenalectomy compared with survivors treated without adrenalectomy, and compared with healthy controls. Other treatment components did not affect the increased basal cortisol and ACTH levels. In the normal population, cortisol levels are known to be highly dependent on physical (e.g. physical activity) or psychosocial (e.g. emotional arousal) stress. It is possible that participating in this study was more stressful for survivors, because of their medical history, than for healthy controls. Raised cortisol levels have been previously reported in childhood cancer survivors after cranial irradiation. ${ }^{24}$ It could be that this is explained by damaging the hypothalamus or pituitary, or that raised cortisol levels are a more universal finding in childhood cancer survivors, either explained by physical or pshycosocial stress. However, if that would be the explanation, similar cortisol and ACTH levels would then have been expected in survivors treated without adrenalectomy. The hypothesis of a higher 'setpoint' of the adrenal system in cases after adrenalectomy is therefore more reasonable. The presence of both high ACTH levels and high cortisol levels in survivors after adrenalectomy suggests that the feedback system has been altered. A possible explanation for this higher setpoint could be 
that it is achieved by a feedback loop of the neural pathways, which might be damaged during surgery. Such a mechanism has been described for the thyroid gland, which is known to have neural connections with the hypothalamic suprachiasmatic and paraventricular nuclei. Although the specific role of autonomic innervation of the thyroid gland in terms of endocrine regulation remains to be elucidated, it is very likely that these connections contribute to the coordination of metabolic control. ${ }^{25}$ Although the pathogenesis of this possible higher setpoint is not clear yet, it is important to realize that the presence of elevated cortisol levels can influence the metabolic system in this subset of survivors. Cortisol plays a role in gluconeogenesis, suppresses the immune system, and is involved in fat, protein and carbohydrate metabolism. Higher prevalence of coronary calcification in young and middle aged adults has been described in subjects with more flattened cortisol slopes, but not with average cortisol levels. ${ }^{26}$ In a large Dutch epidemiologic study total cortisol exposure was associated with higher prevalence of atherosclerosis as indicated by number of plaques in the carotid arteries. ${ }^{27}$ We found baseline serum cortisol levels to be positively associated with lipid concentrations and insulin resistance. This indicates that the higher setpoint of the HPA axis may influence metabolic and cardiovascular parameters in longterm childhood cancer survivors and are therefore important confounders for future studies.

In our study, adrenal function was assessed by a low-dose ACTH stimulation test. This test is considered to be superior to the $250 \mu \mathrm{g}$ short ACTH stimulation test, as $250 \mu \mathrm{g}$ is a supraphysiological dose. This may lead to overstimulation of partially atrophied adrenals, thereby producing a deceivingly adequate cortisol response. ${ }^{20,28}$ So far, no consensus has been reached regarding the cut-off value for peak cortisol levels after the low-dose ACTH stimulation test. According to a meta-analysis by Kazlauskaite et al. cortisol levels below 440 at 30 minutes after administration of Synacthen are predictive of hypothalamic-pituitary-adrenal insufficiency. ${ }^{20}$ The lack of a standard cortisol assay method explains at least partly the variability in diagnostic cortisol thresholds reported across studies. For that reason and also because large control groups of young adults tested for adrenal insufficiency are lacking, we felt that an age-matched control group consisting of healthy subjects was mandatory to strengthen our study. Results of the Synacthen-test in our control subjects confirm that $440 \mathrm{nmol} / \mathrm{l}$ is a correct cut-off value in this group of survivors and controls. Moreover, as $73 \%$ of the control subjects in this study were relatives of the survivors, the possibly confounding effect of genetic variation is negligible.

Although 17-hydroxy-progesterone, adione, DHEA, DHEAs and testosterone have, compared with cortisol, only minor importance in the diagnosis of primary adrenal insufficiency, their levels as a reflection of normal response to the low dose stimulation test provide further proof of sufficient adrenal function and are in line with our observed serum cortisol levels. In our study, levels of these steroids correlated well with cortisol in males, however in females this correlation was less strong. This might be explained by the fact that we did not perform the Synacthen-test on the same day of the menstrual cycle of the females. It is known that the menstrual cycle yields a varying ovarian contribution to the concentration of these steroids. ${ }^{29}$ 
In conclusion, long-term nephroblastoma and neuroblastoma survivors after unilateral adrenalectomy are not at risk for adrenal insufficiency. However, we found their baseline cortisol and ACTH levels to be elevated compared with controls and survivors without adrenalectomy. This higher setpoint can induce consequences for the metabolic system and cardiovascular risk in these survivors, which is suggested by our findings of elevated lipid levels and increased insulin resistance. Our study indicates that further studies on the exact mechanism behind the altered setpoint of the adrenal system after adrenalectomy are warranted. Detailed information on the status of the adrenal glands after surgery is therefore of value in those studies. Moreover, studies on the influence of this higher baseline setting on metabolic and cardiovascular parameters in subsets of long-term adult survivors of childhood cancer are relevant. 


\section{Supplementary tables}

Supplementary table 1. Serum cortisol levels in nephroblastoma and neuroblastoma survivors.

\begin{tabular}{llcccccc}
\hline & & $\begin{array}{c}\text { Nephroblastoma } \\
(\mathbf{N}=67)\end{array}$ & $\begin{array}{c}\text { Neuroblastoma } \\
(\mathbf{N}=\mathbf{3 4 )}\end{array}$ & $\begin{array}{c}\text { Controls } \\
(\mathbf{N}=49)\end{array}$ & P-value $^{\mathrm{d}}$ & P-value $^{\mathrm{e}}$ P-value $^{\mathrm{f}}$ \\
\hline Cortisol (nmol/l) & $T=0^{\mathrm{a}}$ & $461(199)$ & $429(185)$ & $399(211)$ & 0.44 & 0.11 & 0.50 \\
& $T=30^{\mathrm{b}}$ & $674(145)$ & $649(141)$ & $662(168)$ & 0.44 & 0.70 & 0.72 \\
& $T=60^{\mathrm{c}}$ & $590(166)$ & $549(153)$ & $565(202)$ & 0.27 & 0.48 & 0.72 \\
\hline
\end{tabular}

Data are expressed as mean (standard deviation). ${ }^{\mathrm{a}} \mathbf{T}=\mathbf{0}=$ baseline, ${ }^{\mathrm{b}} \mathbf{T}=\mathbf{3 0}=30$ minutes after administration of Synacthen. ${ }^{\mathrm{c}} \mathbf{T}=\mathbf{6 0}=60$ minutes after administration of Synacthen. ${ }^{\mathrm{d}}$ Nephroblastoma survivors compared with neuroblastoma survivors. eNephroblastoma survivors compared with controls. ${ }^{\mathrm{f}} \mathrm{Neuroblastoma}$ survivors compared with controls.

Supplementary table 2. Steroids in survivors and controls.

\begin{tabular}{|c|c|c|c|c|c|}
\hline & & Controls & $\begin{array}{c}\text { Survivors no } \\
\text { adrenalectomy }\end{array}$ & $\begin{array}{c}\text { Survivors } \\
\text { adrenalectomy }\end{array}$ & P-value $^{d}$ \\
\hline \multirow[t]{3}{*}{ DHEA (nmol/l) } & $T=0^{\mathrm{a}}$ & $26.0(17.0-48.7)$ & $24.4(13.7-36.0)$ & $34.4(18.2-52.5)$ & 0.62 \\
\hline & $T=30^{\mathrm{b}}$ & $45.9(30.3-79.0)$ & $46.4(29.2-68.7)$ & $48.1(30.8-88.6)$ & 0.85 \\
\hline & $T=60^{c}$ & $32.5(22.8-56.9)$ & $33.6(19.8-55.0)$ & $31.5(21.0-69.0)$ & 0.97 \\
\hline \multirow[t]{3}{*}{ DHEAs $(\mu \mathrm{mol} / \mathrm{l})$} & $T=0^{\mathrm{a}}$ & $4.8(3.0-7.0)$ & $4.4(2.4-6.6)$ & $5.0(2.6-8.6)$ & 0.81 \\
\hline & $T=30^{\mathrm{b}}$ & $5.1(3.9-5.7)$ & $4.6(2.5-6.9)$ & $5.1(2.7-8.1)$ & 0.69 \\
\hline & $T=60^{c}$ & $4.9(3.2-6.7)$ & $4.5(2.5-7.1)$ & $5.7(2.7-8.6)$ & 0.50 \\
\hline \multirow[t]{3}{*}{ Adione (nmol/l) } & $T=0^{\mathrm{a}}$ & $6.6(5.3-9.3)$ & $6.5(4.3-9.0)$ & $7.7(5.0-11.0)$ & 0.38 \\
\hline & $T=30^{\mathrm{b}}$ & $9.2(6.3-11.6)$ & $8.7(6.4-11.0)$ & $9.3(6.0-12.6)$ & 0.88 \\
\hline & $T=60^{c}$ & $7.6(5.8-9.8)$ & $8.0(5.3-9.8)$ & $7.6(5.3-11.1)$ & 0.60 \\
\hline \multirow[t]{3}{*}{$170 \mathrm{HP}(\mathrm{nmol} / \mathrm{l})$} & $T=0^{a}$ & $3.2(2.1-4.9)$ & $2.9(1.5-4.3)$ & $3.3(1.8-4.8)$ & 0.82 \\
\hline & $T=30^{\mathrm{b}}$ & $5.1(3.8-5.7)$ & $5.1(3.8-7.0)$ & $4.2(3.0-5.3)$ & 0.05 \\
\hline & $T=60^{c}$ & $3.5(2.4-4.6)$ & $3.4(2.3-4.9)$ & $3.1(2.0-4.6)$ & 0.43 \\
\hline \multirow[t]{3}{*}{ Testosterone (females) (nmol/l) } & $T=0^{a}$ & $0.9(0.6-1.4)$ & $0.7(0.5-1.4)$ & $0.7(0.5-1.3)$ & 0.88 \\
\hline & $T=30^{\mathrm{b}}$ & $1.2(0.7-1.7)$ & $0.8(0.6-1.5)$ & $1.0(0.7-1.5)$ & 0.60 \\
\hline & $T=60^{c}$ & $1.2(0.6-1.5)$ & $0.8(0.7-1.6)$ & $0.9(0.7-1.5)$ & 0.63 \\
\hline
\end{tabular}

Data are expressed as median (interquartile range). ${ }^{\mathrm{a}} \mathbf{T}=\mathbf{0}=$ baseline, ${ }^{\mathrm{b}} \mathbf{T}=\mathbf{3 0}=30$ minutes after administration of Synacthen. ${ }^{\mathrm{C}} \mathbf{T}=\mathbf{6 0}=\mathbf{6 0}$ minutes after administration of Synacthen. ${ }^{\mathrm{d}}$ Survivors after adrenalectomy compared with controls. Adione = androstenedione; DHEA = Dehydro-epiandosterone; DHEAs = dehydroepiandosterone-sulfate; $170 H \mathbf{P}=$ 17-Hydroxyprogesterone. 
Supplementary table 3 . Steroids in survivors.

\begin{tabular}{|c|c|c|c|c|c|}
\hline & & Males & $\begin{array}{l}\text { Females } \\
\text { non OAC }\end{array}$ & $\begin{array}{c}\text { Females } \\
\text { OAC }\end{array}$ & P-value $^{d}$ \\
\hline \multirow[t]{3}{*}{ DHEA (nmol/l) } & $T=0^{\mathrm{a}}$ & $34.8(21.0-59.3)$ & $16.4(9.1-27.9)$ & $23.6(13.8-36.4)$ & 0.30 \\
\hline & $T=30^{\mathrm{b}}$ & $54.9(38.6-87.8)$ & $34.5(18.7-53.2)$ & $40.7(30.6-74.4)$ & 0.86 \\
\hline & $T=60^{c}$ & $37.9(26.1-67.2)$ & $21.9(14.8-35.0)$ & $30.2(18.9-43.5)$ & 0.65 \\
\hline \multirow[t]{3}{*}{ DHEAs ( $\mu \mathrm{mol} / \mathrm{I})$} & $T=0^{\mathrm{a}}$ & $5.6(3.9-10.1)$ & $2.8(1.1-5.1)$ & $3.3(2.5-5.3)$ & 0.58 \\
\hline & $T=30^{\mathrm{b}}$ & $5.7(3.8-9.1)$ & $3.0(1.3-6.0)$ & $3.7(2.2-5.3)$ & 0.49 \\
\hline & $T=60^{c}$ & $6.1(4.0-9.8)$ & $2.9(1.3-5.8)$ & $3.6(2.5-5.7)$ & 0.47 \\
\hline \multirow[t]{3}{*}{ Adione (nmol/l) } & $T=0^{\mathrm{a}}$ & $8.3(6.1-10.2)$ & $4.8(3.3-7.6)$ & $6.1(4.0-9.0)$ & 0.87 \\
\hline & $T=30^{\mathrm{b}}$ & $10.5(7.9-13.3)$ & $6.7(5.0-9.3)$ & $5.6(4.5-9.8)$ & 0.18 \\
\hline & $T=60^{c}$ & $8.6(6.5-10.9)$ & $6.1(4.5-8.8)$ & $6.3(4.5-9.4)$ & 0.26 \\
\hline \multirow[t]{3}{*}{$170 H P(n m o l / l)$} & $T=0^{\mathrm{a}}$ & $3.8(2.5-4.6)$ & $2.5(0.9-4.4)$ & $1.4(0.8-2.0)$ & 0.048 \\
\hline & $T=30^{\mathrm{b}}$ & $4.9(3.9-7.1)$ & $4.7(3.5-6.3)$ & $2.8(2.1-3.3)$ & $<0.001$ \\
\hline & $T=60^{c}$ & $3.9(2.8-5.5)$ & $3.3(1.7-4.9)$ & $1.6(1.3-2.2)$ & $<0.001$ \\
\hline \multirow[t]{3}{*}{ Testosterone (females) (nmol/l) } & $T=0^{\mathrm{a}}$ & n.a. & $0.7(0.5-1.3)$ & $0.7(0.5-1.7)$ & 0.44 \\
\hline & $T=30^{\mathrm{b}}$ & n.a. & $1.0(0.7-1.4)$ & $0.8(0.4-1.7)$ & 0.20 \\
\hline & $T=60^{c}$ & n.a. & $0.8(0.7-1.5)$ & $0.7(0.4-1.6)$ & 0.15 \\
\hline
\end{tabular}

Data are expressed as median (interquartile range). ${ }^{\mathrm{a}} \mathbf{T}=\mathbf{0}=$ baseline, ${ }^{\mathrm{b}} \mathbf{T}=\mathbf{3 0}=\mathbf{3 0}$ minutes after administration of Synacthen. ${ }^{\mathrm{C}} \mathbf{T}=\mathbf{6 0}=\mathbf{6 0}$ minutes after administration of SyOnacthen. ${ }^{\mathrm{d} F e m a l e s}$ using oral estrogens compared with females not using oral estrogens. Adione= androstenedione, DHEA = Dehydro-epiandosterone, DHEAs $=$ dehydroepiandosterone-sulfate, 170HP =17-Hydroxyprogesterone. 


\section{References}

1. Hewitt M. WSL, Simone J.V., ed. Childhood cancer survivorship: improving care and quality of life. Washington, DC: National Academies Press 2003.

2. Wright KD, Green DM, Daw NC. Late effects of treatment for wilms tumor. Pediatr Hematol Oncol 2009;26:407-13.

3. van Dijk IW, Oldenburger F, Cardous-Ubbink MC, et al. Evaluation of late adverse events in long-term wilms' tumor survivors. Int J Radiat Oncol Biol Phys 2010;78:370-8.

4. Laverdiere C, Liu Q, Yasui Y, et al. Long-term outcomes in survivors of neuroblastoma: a report from the Childhood Cancer Survivor Study. J Natl Cancer Inst 2009;101:1131-40.

5. Green DM, Kawashima T, Stovall M, et al. Fertility of female survivors of childhood cancer: a report from the childhood cancer survivor study. J Clin Oncol 2009;27:2677-85.

6. Green DM, Kawashima T, Stovall M, et al. Fertility of male survivors of childhood cancer: a report from the Childhood Cancer Survivor Study. J Clin Oncol 2010;28:332-9.

7. Hudson MM. Reproductive outcomes for survivors of childhood cancer. Obstet Gynecol 2010;116:117183.

8. Lie Fong S, Laven JS, Hakvoort-Cammel FG, et al. Assessment of ovarian reserve in adult childhood cancer survivors using anti-Mullerian hormone. Human Reprod (Oxford, England) 2009;24:982-90.

9. van Casteren NJ, van der Linden GH, Hakvoort-Cammel FG, Hahlen K, Dohle GR, van den Heuvel-Eibrink MM. Effect of childhood cancer treatment on fertility markers in adult male long-term survivors. Pediatr Blood Cancer 2009;52:108-12.

10. Steffens $M$, Beauloye V, Brichard B, et al. Endocrine and metabolic disorders in young adult survivors of childhood acute lymphoblastic leukemia (ALL) or non-Hodgkin lymphoma (NHL). Clin Endocrinol (Oxf) 2008;69:819-27.

11. van Beek RD, de Muinck Keizer-Schrama SM, Hakvoort-Cammel FG, et al. No difference between prednisolone and dexamethasone treatment in bone mineral density and growth in long term survivors of childhood acute lymphoblastic leukemia. Pediatr Blood Cancer 2006;46:88-93.

12. van Beek RD, van den Heuvel-Eibrink MM, Hakvoort-Cammel FG, et al. Bone mineral density, growth, and thyroid function in long-term survivors of pediatric Hodgkin's lymphoma treated with chemotherapy only. J Clin Endocrinol Metab 2009;94:1904-9.

13. van der Sluis IM, van den Heuvel-Eibrink MM, Hahlen K, Krenning EP, de Muinck Keizer-Schrama SM. Bone mineral density, body composition, and height in long-term survivors of acute lymphoblastic leukemia in childhood. Med Pediatr Oncol 2000;35:415-20.

14. van Waas M, Neggers SJ, Pieters R, van den Heuvel-Eibrink MM. Components of the metabolic syndrome in 500 adult long-term survivors of childhood cancer. Ann Oncol 2010;21:1121-6.

15. van Waas $M$, Neggers SJ, van der Lelij AJ, Pieters R, van den Heuvel-Eibrink MM. The metabolic syndrome in adult survivors of childhood cancer, a review. J Pediatr Hematol Oncol 2010;32:171-9.

16. Mertens AC, Liu Q, Neglia JP, et al. Cause-specific late mortality among 5-year survivors of childhood cancer: the Childhood Cancer Survivor Study. Journal of the National Cancer Institute 2008;100:136879.

17. Burke CW. Adrenocortical insufficiency. Clin Endocrinol Metab 1985;14:947-76.

18. Bergthorsdottir R, Leonsson-Zachrisson M, Oden A, Johannsson G. Premature mortality in patients with Addison's disease: a population-based study. J Clin Endocrinol Metab 2006;91:4849-53.

19. Assembly WMAG. Declaration of Helsinki - Sixth revision. 2008.

20. Kazlauskaite R, Evans AT, Villabona CV, et al. Corticotropin tests for hypothalamic-pituitary- adrenal insufficiency: a metaanalysis. J Clin Endocrinol Metab 2008;93:4245-53.

21. Matthews DR, Hosker JP, Rudenski AS, Naylor BA, Treacher DF, Turner RC. Homeostasis model assessment: insulin resistance and beta-cell function from fasting plasma glucose and insulin concentrations in man. Diabetologia 1985;28:412-9. 
22. Veldhuis JD, Iranmanesh A, Roelfsema F, et al. Tripartite control of dynamic ACTH-cortisol dose responsiveness by age, body mass index, and gender in 111 healthy adults. J Clin Endocrinol Metab 2011;96:2874-81.

23. Bright GM. Corticosteroid-binding globulin influences kinetic parameters of plasma cortisol transport and clearance. J Clin Endocrinol Metab 1995;80:770-5.

24. Darzy KH, Shalet SM. Absence of adrenocorticotropin (ACTH) neurosecretory dysfunction but increased cortisol concentrations and production rates in ACTH-replete adult cancer survivors after cranial irradiation for nonpituitary brain tumors. J Clin Endocrinol Metab 2005;90:5217-25.

25. Fliers E, Klieverik LP, Kalsbeek A. Novel neural pathways for metabolic effects of thyroid hormone. Trends Endocrinol Metab 2010;21:230-6.

26. Matthews K, Schwartz J, Cohen S, Seeman T. Diurnal cortisol decline is related to coronary calcification: CARDIA study. Psychosom Med 2006;68:657-61.

27. Dekker MJ, Koper JW, van Aken MO, et al. Salivary cortisol is related to atherosclerosis of carotid arteries. J Clin Endocrinol Metab 2008;93:3741-7.

28. Abdu TA, Elhadd TA, Neary R, Clayton RN. Comparison of the low dose short synacthen test (1 microg), the conventional dose short synacthen test (250 microg), and the insulin tolerance test for assessment of the hypothalamo-pituitary-adrenal axis in patients with pituitary disease. J Clin Endocrinol Metab 1999;84:838-43.

29. Aedo AR, Landgren BM, Diczfalusy E. Studies on ovarian and adrenal steroids at different phases of the menstrual cycle: II. A comparative assessment of the circadian variation in steroid and lutropin levels during the follicular, periovulatory and luteal phases. Contraception 1981;23:407-24. 



\section{Abstract}

Background: The risk of metabolic late effects after childhood cancer, such as obesity, hypertension and diabetes, can be positively influenced by a healthy lifestyle with sufficient physical activity. Nevertheless, studies on physical activity in adult survivors of childhood cancer are scarce and involve different and often non-validated questionnaires. Aim of the study was to assess daily life physical activity in Dutch adult long-term nephroblastoma and neuroblastoma survivors.

Patients and methods: To assess physical activity, the Short QUestionnaire to ASsess Healthenhancing physical activity (SQUASH), which was developed and validated to assess daily life physical activity in the Dutch adult population, was used. Sixty-seven nephroblastoma and 36 neuroblastoma survivors (median age 30 years, range 18 -51) and 60 socio-demographically similar healthy control subjects (median age 32 years, range $18-61$ ) were asked to complete the SQUASH during their regular follow-up visit.

Results: The adjusted mean physical activity score in male neuroblastoma survivors (mean 7767, $\mathrm{P}=0.028$ ) was significantly lower than male controls (mean 10341), whereas it was not significantly lower in male nephroblastoma survivors (mean 8980, $\mathrm{P}=0.121$ ). Adjusted means for physical activity scores in females were not different from their controls.

Conclusion: Male neuroblastoma survivors were identified as performing less daily physical activity. 


\section{Introduction}

Survival of nephroblastoma and neuroblastoma has increased significantly during the last few decades, $92 \%$ and $55 \%$ respectively. This increased survival is due to better stratification, improvements in treatment strategies and optimized supportive care. With a mean survival rate, nephroblastoma and neuroblastoma survivors represent a considerable group of childhood cancer survivors. ${ }^{1,2}$

Pulmonary, muscoskeletal, renal, and cardiac late effects, but also second malignant neoplasms and increased risk of cardiovascular diseases, have been reported in these survivors. ${ }^{3-6}$ Some of these late effects influencing cardiovascular risk, for example hypertension, obesity, dyslipidemia and insulin resistance, which altogether represent the metabolic syndrome, might be positively affected by sufficient physical activity.

In a recent review, most studies reported a lower activity status of childhood cancer survivors as compared with control subjects. Survivors treated with cranial radiotherapy or amputation are less physically active. ${ }^{7}$ Limitations of previously performed research on physical activity in childhood cancer survivors are the lack of objective physical activity measures, validated questionnaires, non-cancer comparison groups and the heterogeneity of studied diagnostic groups. $^{7}$

The Short Questionnaire to Assess Health-enhancing physical activity (SQUASH) was developed by the Dutch National Institute of Public Health and the Environment in order to assess daily life physical activity in the Dutch adult population. ${ }^{8}$ The SQUASH contains questions about commuting activities, exertion at work or school, household activities and leisure time and sports during the last week. Although this questionnaire is a subjective measure of physical activity, it has an important advantage over other questionnaires because it was validated using an objective measure: a Computer Science and Applications (CSA) activity monitor.

The objective of this study was to assess daily life physical activity using the SQUASH and to assess compliance to the Dutch physical activity guidelines in Dutch adult long-term survivors of nephroblastoma and neuroblastoma compared to a non-cancer comparison group.

\section{Patients and methods}

\section{Participants}

All long-term (i.e. $\geq 5$ years after cessation of treatment) adult survivors of childhood nephroblastoma and neuroblastoma, treated between 1961 and 2004 in the Erasmus MC-Sophia Children's Hospital routinely visiting the late effects outpatient clinic were invited to participate in this study. Informed consent was obtained according to the Helsinki declaration ${ }^{9}$ and the study was approved by the local medical ethical committee. Adult survivors of neuroblastoma stage $4 \mathrm{~S}$ who did not receive any treatment were excluded from this study. During their regular 
visit, history was taken and physical examination, laboratory tests and imaging studies were performed. Additionally, survivors were asked to introduce a healthy control subject who had not been treated for childhood cancer to participate in the study. Brothers and sisters were preferred, otherwise a friend or partner with a maximum age difference of five years was eligible. This control group was designed as a socio-demographically similar comparison population. The study took part from October 2009 until March 2011.

\section{Outcome of interest}

Information about daily life physical activity was collected with the SQUASH. ${ }^{8}$ The SQUASH expresses physical activity as a physical activity score. Physical activity score is the sum of activity scores for each question. A higher physical activity score indicates a higher amount of daily physical activity. Questions are pre-structured in commuting activities, activities at work or school, household activities and leisure time activities including sports. The SQUASH consists of three main queries about physical activity: days per week, average time per day and intensity. Intensity is subdivided in three categories based on Ainsworth's Compendium of Physical Activities. ${ }^{10}$ The method of Wendel-Vos et al. was used for allocating intensity scores to reported activities. ${ }^{8}$ For determining intensity categories of reported sports, the method of Ainsworth et al. ${ }^{10}$ was used as described before. ${ }^{8}$ Physical activity score is the sum of activity scores for each question. Mean physical activity score using the SQUASH was reported to be 7850 in a study of healthy males and females (mean age 44 years, standard deviation 6). To assess compliance to the Dutch guidelines for physical activity, ${ }^{11}$ the question 'How many days a week are you bicycling, doing odd jobs, gardening or exercising for at least 30 minutes?' was added. If subjects answered this question with five days or more, they were considered to adhere to the guideline. ${ }^{11}$

\section{Data collection}

Information on disease and treatment was obtained from our local database and medical records. To assess the influence of relevant confounders, the following data were collected: Information regarding smoking status and socio-economic status was collected using a selfdesigned questionnaire. Smoking status was categorized as non-smoker, former smoker and current smoker. Socio-economic status was defined by the highest level of educational attainment and based on the Dutch educational system. Height was measured to the nearest millimeter using a Harpenden Stadiometer and weight was measured to the nearest 0.1 kilogram on a standard clinical balance. Body mass index (BMI) was calculated as weight $(\mathrm{kg}) / \mathrm{height}^{2}(\mathrm{~m})$. Spinal deformities were determined in survivors by physical examination. $\mathrm{N}$-terminal pro-brain natriuretic peptide (NT-pro-BNP) was determined as a marker for heart function in both survivors and control subjects and was measured using a validated, commercially available immunoassay (Elecsysw ProBNP, Roche Diagnostics, Indianapolis, IN, USA), using established methodology. ${ }^{12}$ 


\section{Statistics}

Statistical analyses were performed with the Statistical Package for Social Sciences 18.0 (SPSS Inc., Chicago, IL, USA). Mann-Whitney U tests were used to compare physical activity scores, BMI, and NT-pro-BNP between survivors and control subjects. Before performing adjusted subgroup comparisons, missing data were imputed according to the following strategy: when less than $5 \%$ of data were missing, the median value was imputed as described previously. ${ }^{13}$ When more than $5 \%$ of data were missing, customized models were developed to predict the predictor from all the other predictors. Adjusted subgroup comparisons for sex and diagnosis subgroups were performed by calculating the estimated marginal means of the factor scores of the clusters, with adjustments for the confounders age, sex, smoking, socio-economic status, NT-pro-BNP and BMI. Multiple linear regression analysis was performed to assess the influence of spinal deformities on physical acivity in survivors only. P-values of $<0.05$ (two tailed) were considered statistically significant.

\section{Results}

\section{Participants}

Hundred-three adult survivors of nephroblastoma and 54 adult survivors of neuroblastoma who survived at least five years after cessation of treatment and who were registered in our outpatient clinic registry were eligible to participate in the study. Of the 103 survivors of nephroblastoma 28 could not be contacted for participation (six died after having survived at least five years after cessation of treatment, eight refused to attend the clinic, five were lost to follow-up, six emigrated and three visited a outpatient clinic in another pediatric oncology centre). Of the 54 survivors of neuroblastoma 12 could not be contacted for participation (three died after having survived for at least five years after cessation of treatment, three refused to attend the clinic, five were lost to follow-up and one emigrated). In total 75 survivors of nephroblastoma and 42 survivors of neuroblastoma were contacted for participation. Eight nephroblastoma survivors (two being ill at the time, one lost to follow-up and five refused to participate) and six neuroblastoma survivors (three were currently pregnant, two being ill at the time and one refused to participate) did not participate. In total 103 adult survivors (67 nephroblastoma and 36 neuroblastoma survivors) participated in the current study. Additionally, 60 healthy control subjects were included. Ninetyfour percent $(\mathrm{N}=97)$ of the survivors and eighty-three percent $(\mathrm{N}=50)$ of the control subjects answered all the questions of the SQUASH. Median age of nephroblastoma survivors was 30.2 years (range 18.8 - 50.8 years), of neuroblastoma survivors 29.6 years ( $20.4-46.2$ years) and of control subjects 32.1 years (range $18.0-61.7$ years). Median age at diagnosis was 2.3 years (range $0.0-12.7$ years) and median follow-up time after cessation of treatment was 26.2 years (range $6.4-48.9$ years) in nephroblastoma survivors and 27.8 years (range $15.0-44.4$ years) in neuroblastoma survivors. Characteristics of the study participants are shown in Table 1. 
Table 1. Baseline characteristics.

\begin{tabular}{|c|c|c|c|}
\hline & Nephroblastoma & Neuroblastoma & Controls \\
\hline $\mathrm{N}$ & 67 & 36 & 60 \\
\hline Male/female (N) & $39 / 28$ & $15 / 21$ & $27 / 33$ \\
\hline Age at follow-up (yrs) & $30.2(18.8-50.8)$ & $29.6(20.4-46.2)$ & $32.1(18.0-61.8)$ \\
\hline Age at diagnosis (yrs) & $3.3(0.0-12.7)$ & $0.8(0.0-11.7)$ & n.a. \\
\hline Follow-up time (yrs) & $26.2(6.4-48.9)$ & $27.8(15.0-44.4)$ & n.a. \\
\hline \multicolumn{4}{|l|}{ Stage $(N)$} \\
\hline I & 32 & 6 & n.a. \\
\hline il & 13 & 17 & \\
\hline III & 11 & 8 & \\
\hline IV & 3 & 2 & \\
\hline V & 1 & 0 & \\
\hline unknown & 7 & 3 & \\
\hline Surgery (N) & 67 & 36 & n.a. \\
\hline radiotherapy $(\mathrm{N})$ & 35 & 12 & n.a. \\
\hline cumulative dose (Gy) & $25(15-40)$ & $20(10-30)$ & n.a. \\
\hline chemotherapy $(\mathrm{N})$ & 59 & 31 & n.a. \\
\hline \multicolumn{4}{|l|}{ smoking } \\
\hline - non smoking & $65.7 \%(44)$ & $47.2 \%(17)$ & $46.7 \%(28)$ \\
\hline - former smoker & $11.9 \%(8)$ & $22.2 \%(8)$ & $16.7 \%(10)$ \\
\hline - current smoker & $22.4 \%(15)$ & $30.6 \%(11)$ & $31.7 \%(19)$ \\
\hline missing & $0.0 \%(0)$ & $0.0 \%(0)$ & $5.0 \%(3)$ \\
\hline \multicolumn{4}{|l|}{ Socio-economic status } \\
\hline - Low & $6.0 \%(4)$ & $2.8 \%(1)$ & $1.7 \%(1)$ \\
\hline - Intermediate & $46.3 \%(31)$ & $47.2 \%(17)$ & $55.0 \%(33)$ \\
\hline - High & $47.8 \%(32)$ & $50.0 \%(18)$ & $40.0 \%(24)$ \\
\hline missing & $0.0 \%(0)$ & $0.0 \%(0)$ & $3.3 \%(2)$ \\
\hline
\end{tabular}

Data are expressed as median (range) or as $\%(\mathrm{~N})$.

\section{Outcomes}

Physical activity scores in nephroblastoma survivors (median 8140, $\mathrm{P}=0.721$ ) were not different from controls (median 8080), whereas neuroblastoma survivors had low physical activity scores compared with controls (median 6685, $\mathrm{P}=0.043$ ). Physical activity scores in female survivors of both nephroblastoma (median 8490, $\mathrm{P}=0.205$ ) and neuroblastoma (median 7190, $\mathrm{P}=0.979$ ) were not significantly different from sex-matched controls (median 7200), nor was physical activity score of male nephroblastoma survivors (median 8040 vs. 9528, $\mathrm{P}=0.442$ ). Male survivors of 
neuroblastoma however reported a significantly lower physical activity score than male control subjects (median 6390 versus 9528, $\mathrm{P}=0.031$ ) (Table 2 ). Female nephroblastoma survivors seem to adhere more often to the physical activity guidelines (at least 30 minutes of activity per day at least 5 days a week) than female controls, however this difference was not significant $(64.3 \%$ vs. $44.4 \%, \mathrm{P}=0.180$ ).

Adherence to the guideline was comparable between female neuroblastoma survivors and female controls ( $47.6 \%$ vs. $44.4 \%, \mathrm{P}=0.920$ ) (Table 2 ). Male survivors of nephroblastoma (35.9\%, $\mathrm{P}=0.066)$ and male survivors of neuroblastoma $(33.3 \%, \mathrm{P}=0.119)$ seemed to adhere less frequently to the guidelines than male controls (57.5\%), however these differences were not significant (Table 2). There were no differences in median BMI and NT-pro-BNP between survivors and control subjects (Table 2), however both nephroblastoma and neuroblastoma survivors more often had high (>13.5 pmol/I) NT-pro-BNP levels (Table 2). Spinal deformities were present in 43/92 (42\%) assessed survivors.

After correction for the confounding effects of age, sex, socio-economic status, smoking, NT-proBNP and BMI, the adjusted mean physical activity scores between female controls (7808), female nephroblastoma survivors (9327) and neuroblastoma survivors (7969) were not significantly different (Figure 1). The adjusted mean physical activity score in male neuroblastoma survivors was significantly lower compared with male controls (mean 7205 vs. mean 10341, $\mathrm{P}=0.006$ ). Physical activity score in male nephroblastoma survivors also seemed to be lower than in controls, however this difference was not significant (mean 8980 vs. mean 10341, $P=0.121$ ) (Figure 2). Physical activity scores in male control subjects were significantly higher compared with female control subjects $(P=0.020)$. To assess the influence of spinal deformities on physical activity score we performed a multiple linear regression analysis in survivors only, correcting for age, sex, smoking, socio-economic status, NT-proBNP and BMI. The results showed no associations between activity score and spinal deformities $(\beta=1219, P=0.116)$. 


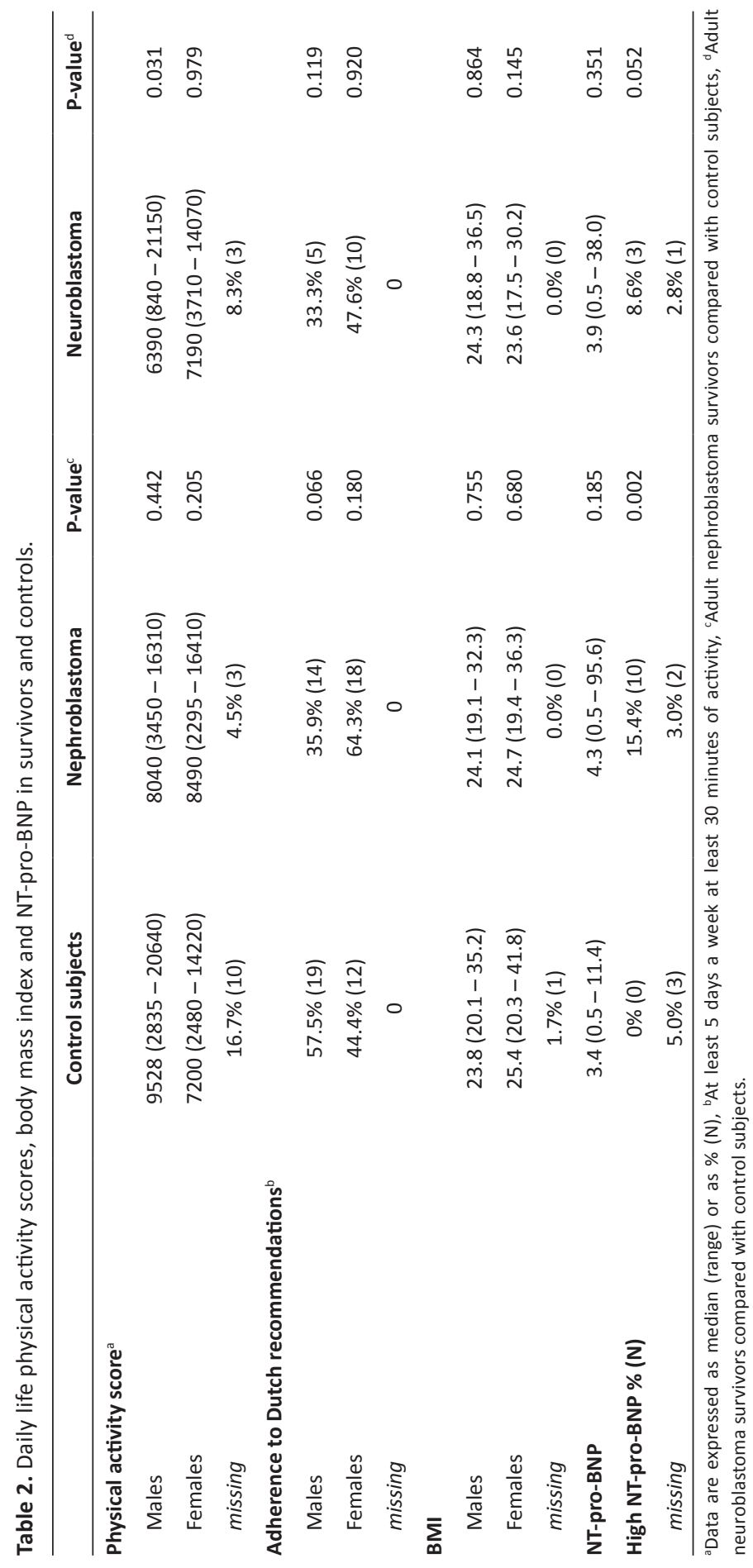




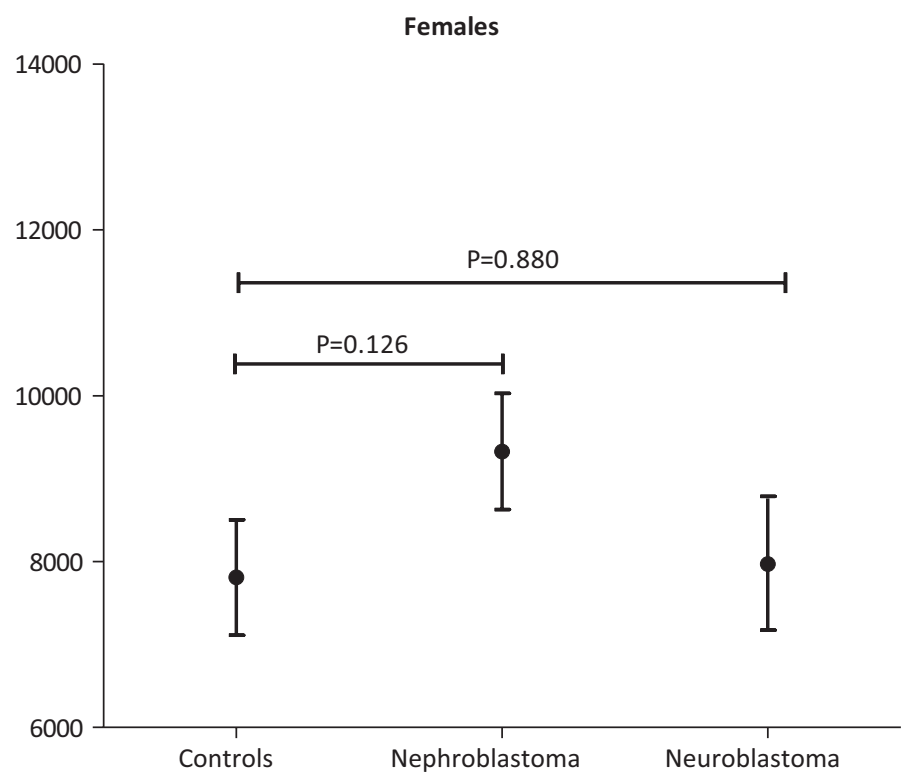

Figure 1. Adjusted mean physical activity scores in females.

Adjusted means are corrected for age, sex, smoking, socio-economic status, NT-pro-BNP and BMI. Y-axis runs from 6000 to 14000.

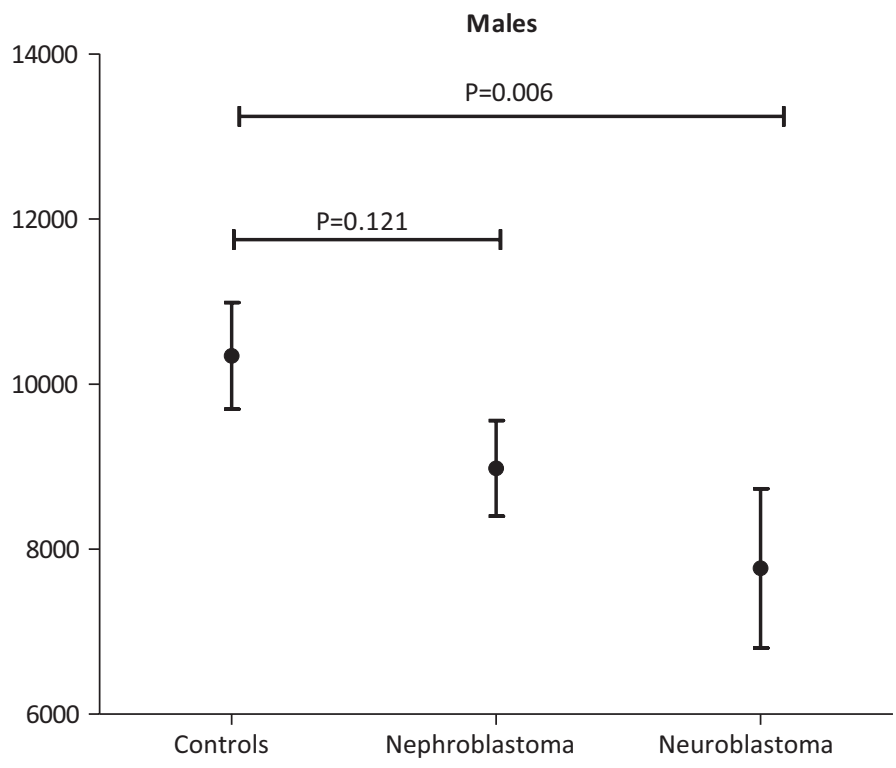

Figure 2. Adjusted mean physical activity scores in males.

Adjusted means are corrected for age, sex, smoking, socio-economic status, NT-pro-BNP and BMI. Y-axis runs from 6000 to 14000. 


\section{Discussion}

We investigated daily life physical activity in 67 nephroblastoma and 36 neuroblastoma survivors compared to 60 socio-demographically comparable healthy control subjects, using the SQUASH. After correction for age, sex, smoking status, socio-economic status, BMI and NT-pro-BNP, male survivors of neuroblastoma reported less daily life activity than male control subjects.

It is unknown why male neuroblastoma survivors are less physically active than their healthy peers. Specific late effects of cancer treatment could possibly induce a less active lifestyle. Cardiac late effects and deformities such as scoliosis may influence daily life physical activity, but in our study, spinal deformities and NT-pro-BNP did not have such an effect.

Physical activity scores in female survivors were not different from female control subjects. It is possible that female survivors are more concerned about their health status and therefore commit more often in physical activity. Compared with the male control subjects, female control subjects were less physically active. This is consistent with previous reports which showed that healthy males are more physically active than females both on a daily basis and based on committing to sports. ${ }^{14,15}$ Likewise, the percentage of healthy siblings in the Childhood Cancer Survivors Study not meeting the physical activity guidelines was also somewhat higher in females than in males. ${ }^{16}$

Our data concerning male neuroblastoma survivors are in concordance with findings of Ness et al. In contrast to our study, they reported that also female survivors of nephroblastoma and neuroblastoma were more likely to report an inactive lifestyle compared with siblings. Differences in assessment of physical activity but also in health behaviour and lifestyle between countries might contribute to the discrepant findings.

Our study has some important strengths. We used a proven reliable and validated questionnaire to assess daily life physical activity, especially developed for the Dutch adult population. The almost complete follow-up of survivors limits selection bias. Furthermore, we were able to compare physical activity in adult survivors of childhood cancer with an age, sex and sociodemographically similar control group. Moreover, we investigated the influence of several potential confounders on daily life physical activity. Limitations of our study are the relatively small group of survivors and the use of a subjective measurement of physical activity instead of an objective measurement.

In conclusion, using the SQUASH we found that male neuroblastoma survivors report less daily physical activity than controls. Physical activity in male nephroblastoma survivors and female nephroblastoma and neuroblastoma survivors was comparable with that in controls. Future research on physical activity in adult survivors of childhood cancer should investigate large populations of survivors and focus on all types of childhood malignancies. 


\section{References}

1. Bernstein ML, Leclerc JM, Bunin G, et al. A population-based study of neuroblastoma incidence, survival, and mortality in North America. J Clin Oncol 1992;10:323-9.

2. Bernstein L, Linet $M$, Smith $M$, Olshan AF. Cancer incidence and survival among children and adolescents: United States SEER Program 1975-1995, SEER Program. National Cancer Institute 1999 1999:p.79-90.

3. van Dijk IW, Oldenburger F, Cardous-Ubbink MC, et al. Evaluation of late adverse events in long-term wilms' tumor survivors. Int J Radiat Oncol Biol Phys 2010;78:370-8.

4. Geenen MM, Bakker PJ, Kremer LC, Kastelein JJ, van Leeuwen FE. Increased prevalence of risk factors for cardiovascular disease in long-term survivors of acute lymphoblastic leukemia and Wilms tumor treated with radiotherapy. Pediatr Blood Cancer 2010;55:690-7.

5. Laverdiere C, Liu Q, Yasui Y, et al. Long-term outcomes in survivors of neuroblastoma: a report from the Childhood Cancer Survivor Study. J Natl Cancer Inst 2009;101:1131-40.

6. Wright KD, Green DM, Daw NC. Late effects of treatment for wilms tumor. Pediatr Hematol Oncol 2009;26:407-13.

7. Stolley MR, Restrepo J, Sharp LK. Diet and physical activity in childhood cancer survivors: a review of the literature. Ann Behav Med 2010;39:232-49.

8. Wendel-Vos GC, Schuit AJ, Saris WH, Kromhout D. Reproducibility and relative validity of the short questionnaire to assess health-enhancing physical activity. J Clin Epidemiol 2003;56:1163-9.

9. Assembly WMAG. Declaration of Helsinki - Sixth revision. 2008.

10. Ainsworth BE, Haskell WL, Leon AS, et al. Compendium of physical activities: classification of energy costs of human physical activities. Med Sci Sports Exerc 1993;25:71-80.

11. Kemper HGC, Ooijendijk WTM, Stiggelbout M. [Consensus about the Dutch physical activity guideline]. Tijdschr Soc Gezondheidsz 2000;78:180-3.

12. Januzzi JL, van Kimmenade R, Lainchbury J, et al. NT-proBNP testing for diagnosis and short-term prognosis in acute destabilized heart failure: an international pooled analysis of 1256 patients: the International Collaborative of NT-proBNP Study. Eur Heart J 2006;27:330-7.

13. Harrell FE, Jr. Regression Modelling Strategies. Chapter 3. Missing Data: Springer; 2001.

14. Aaron DJ, Kriska AM, Dearwater SR, et al. The epidemiology of leisure physical activity in an adolescent population. Med Sci Sports Exerc 1993;25:847-53.

15. Fairclough SJ, Boddy LM, Hackett AF, Stratton G. Associations between children's socioeconomic status, weight status, and sex, with screen-based sedentary behaviours and sport participation. Int J Pediatr Obes 2009;4:299-305.

16. Ness KK, Leisenring WM, Huang S, et al. Predictors of inactive lifestyle among adult survivors of childhood cancer: a report from the Childhood Cancer Survivor Study. Cancer 2009;115:1984-94. 



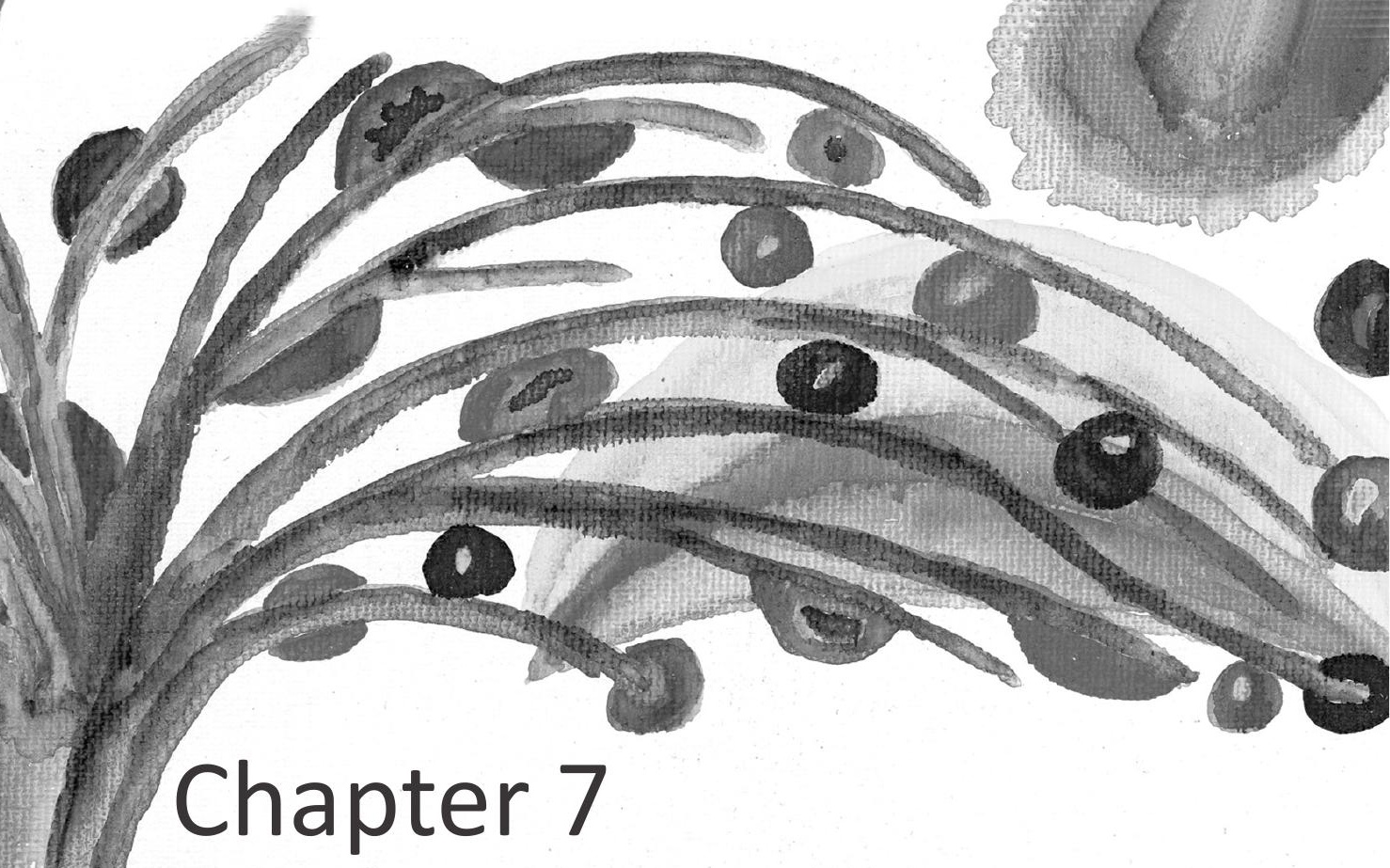

\title{
Abdominal irradiation: An important determinant of metabolic syndrome after nephroblastoma and neuroblastoma
}

\author{
Marjolein van Waas', Sebastian J.C.M.M. Neggers ${ }^{1,2}$, Hein Raat ${ }^{3}$, \\ Caroline M. van Rij ${ }^{4}$, Rob Pieters ${ }^{1}$, Marry M. van den Heuvel-Eibrink ${ }^{1}$
}

1Department of Pediatric Oncology/Hematology, Erasmus MC-Sophia Children's Hospital, Rotterdam, the Netherlands

${ }^{2}$ Department of Medicine, Section Endocrinology, Erasmus University Medical Centre, Rotterdam, the Netherlands

${ }^{3}$ Department of Public Health, Erasmus University Medical Centre, Rotterdam, the Netherlands ${ }^{4}$ Department of Radiation Oncology, Erasmus University Medical Centre, Rotterdam, the Netherlands

Submitted 


\section{Abstract}

Background: Reports on metabolic syndrome in nephroblastoma and neuroblastoma survivors are scarce. Aim was to evaluate the occurrence of and the contribution of treatment regimens to the metabolic syndrome.

Patient and methods: In this prospective study 164 subjects participated (67 long-term nephroblastoma ( 28 females) and 36 neuroblastoma survivors ( 21 females)). Sixty-one control subjects (28 females) were cross-sectionally recruited. Median age at follow-up was 30 (range 19 - 51) years in survivors and 32 (range $18-62$ ) years in controls. Median follow-up time in survivors was 26 range $(6-49)$ years. Waist and hip circumference as well as blood pressure were measured. Body composition and abdominal fat were assessed by dual energy X-ray absorptiometry (DXA-scan). Laboratory measurements included fasting triglyceride, high density lipoprotein-cholesterol (HDL-C), glucose, insulin, low-density lipoprotein-cholesterol (LDL-C) and free fatty acids (FFA) levels.

Results: Nephroblastoma ( $\mathrm{OR}=5.2, \mathrm{P}<0.0001)$ and neuroblastoma ( $\mathrm{OR}=6.5, \mathrm{P}<0.001)$ survivors had more components of the metabolic syndrome than controls. Survivors treated with abdominal irradiation had higher blood pressure, triglycerides, LDL-C, FFA and lower waist circumference. The latter can not be regarded as a reliable factor in these survivors as radiation affects the waist circumference. When total fat percentage was used as a surrogate marker of adiposity the metabolic syndrome was three times more frequent in abdominally irradiated survivors $(27.5 \%)$ than in non-irradiated survivors $(9.1 \%, \mathrm{P}=0.018)$.

Conclusions: Nephroblastoma and neuroblastoma survivors are at increased risk for developing components of metabolic syndrome, especially after abdominal irradiation. We emphasize that survivors treated with abdominal irradiation need alternative adiposity measurements for assessment of metabolic syndrome. 


\section{Introduction}

Survival of childhood cancer has increased significantly over the past few decades, leading to increased recognition and knowledge of late effects. As treatment of childhood cancer is administered in a growing and developing individual, organs and tissues might be affected in a different and more severe way than in adults. The mean five-year survival for nephroblastoma and neuroblastoma survivors is $92 \%$ and $55 \%$, respectively. ${ }^{1-2}$ Although endocrine late sequelae of pediatric cancer treatment are reported, ${ }^{3-7}$ studies in nephroblastoma and neuroblastoma survivors are scarce. Chemotherapy including anthracyclines, actinomycin and cyclophosphamide are administered in a considerable subset of these survivors. Moreover, abdominal radiotherapy is administered which includes the internal organs in the radiation field. In addition, late effects after treatment in these survivors may be aggravated by the removal of the affected organ. Subsequently, development of the metabolic syndrome may occur.

Metabolic syndrome is known to increase the risk for diabetes and cardiovascular diseases. ${ }^{8-9}$ Adipose tissue, or more specifically visceral fat, which is located around the internal organs, plays a central role in the pathophysiology of metabolic syndrome. Adipose tissue has been recognized as a highly active metabolic organ involved in the production of several hormones. ${ }^{10}$ Excess of visceral fat is strongly correlated with cardiovascular diseases, type 2 diabetes, insulin resistance and inflammatory diseases. ${ }^{11-14}$ The easiest way to measure visceral fat is by measuring waist circumference. However, survivors of specific cancer types such as nephroblastoma and neuroblastoma suffer from scarred abdominal areas as a result from surgery and truncal soft tissue hypoplasia caused by abdominal irradiation. ${ }^{15-17}$ It is conceivable that these anatomical derangements induce incorrect interpretation of abdominal adiposity. Subsequently, although this issue has never been raised in childhood cancer survivor studies, underscoring of the frequency of the metabolic syndrome may occur.

Metabolic syndrome is defined for use in a clinical setting, however other metabolic parameters such as measures of insulin resistance and lipids provide additional information about cardiovascular risk. Our primary objective was to evaluate the frequency of (the components of) metabolic syndrome and associated measures of insulin resistance and dyslipidemia in adult long-term childhood nephroblastoma and neuroblastoma survivors in comparison with a control group. Secondary, we aimed to determine the influence of radiotherapy, chemotherapy and surgery on the occurrence of metabolic syndrome. 


\section{Methods}

\section{Patients}

All long-term ( $\geq 5$ years after cessation of treatment) adult survivors of childhood nephroblastoma and neuroblastoma, treated between 1961 - 2004 in the Erasmus MC-Sophia Children's Hospital who regularly visit the Late Effects outpatient clinic were invited to participate in this prospective study. Informed consent was obtained according to the Helsinki declaration ${ }^{18}$ and the study was approved by the local medical ethical committee (NTR 2814). A control group, consisting of siblings, friends or neighbours, preferably of the same sex and within an age range of five years of the survivor, was cross-sectionally recruited.

\section{Methods}

Disease and treatment data were obtained from our local database. Data regarding (partial) nephrectomy and (partial) unilateral adrenalectomy were confirmed from pathological reports. Abdominal radiotherapy was categorized according to location, i.e.: A) spine plus $1.5 \mathrm{~cm}$ on the right and on the left hemi-abdomen, B) left hemi-abdomen including the spine plus $1.5 \mathrm{~cm}$ on the right hemi-abdomen, $C$ ) right hemi-abdomen including the spine plus $1.5 \mathrm{~cm}$ on the left hemi-abdomen, D) total abdomen. Consequently, the following categories according to organs involved in the radiation field were created: Total pancreas $(B+D)$, part of pancreas $(A+C)$, total liver $(C+D)$ and part of liver ( $A+B)$ (Figure 1). Information regarding smoking status, educational level, statine and antihypertensive medication and diabetes was collected using a self-designed questionnaire. Smoking status was defined as non-smoker, former smoker or current smoker. Educational level was defined by the highest level of educational attainment as selected from three categories based on the Dutch educational system. Daily life physical activity was assessed by the Short Questionnaire to Assess Health-enhancing physical activity (SQUASH). ${ }^{19}$ Height was measured to the nearest millimeter using a Harpenden Stadiometer and weight was assessed wearing underwear only to the nearest $0.1 \mathrm{~kg}$ with a standard clinical balance. Body mass index (BMI) was calculated (weight $(\mathrm{kg})$ )/(height $\left.(\mathrm{cm})^{2}\right) \cdot{ }^{20}$ Waist and hip circumference were measured to the nearest $1 \mathrm{~cm}$, midway between last rib and the iliac crest and the maximum circumference of the buttocks, respectively. ${ }^{21}$ Waist-hip-ratio was calculated. Blood pressure was measured with the subject in sitting position after an hour of rest on the right arm with the Dinamap ${ }^{\circledR}$ Procare and was defined as the mean of three measurements. According to the revised National Cholesterol Education Program (NCEP) criteria, participants with at least three of the following components were diagnosed with metabolic syndrome: Waist circumference $>102 \mathrm{~cm}$ in males or $>88 \mathrm{~cm}$ in females; triglycerides $\geq 1.7 \mathrm{mmol} / \mathrm{l}$ or treatment for dyslipidemia; high density lipoproteincholesterol (HDL-C) $<1.03 \mathrm{mmol} / \mathrm{l}$ in males or $<1.30 \mathrm{mmol} / \mathrm{l}$ in females; fasting plasma glucose $\geq 5.6 \mathrm{mmol} / \mathrm{I}$ or treatment for type 2 diabetes; blood pressure $\geq 130 / 85 \mathrm{mmHg}$ or treatment for hypertension. ${ }^{22}$ Data on total body fat mass $(\mathrm{kg})$, lean body mass $(\mathrm{kg})$ and percentage of body fat were retrieved from dual energy X-ray absorptiometry (DXA, GE Lunar Prodigy, USA), which 
was performed in survivors only. In addition, visceral fat percentage was calculated from intraabdominal fat $(\mathrm{kg})$ and total fat $(\mathrm{kg})$ using the DXA scan. ${ }^{23}$ Values for lean body mass and total fat percentage were compared with normal Dutch reference values and calculated as standard deviation scores (SDS). ${ }^{24}$

A

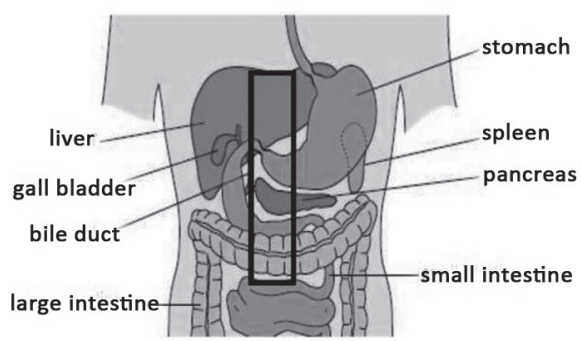

C

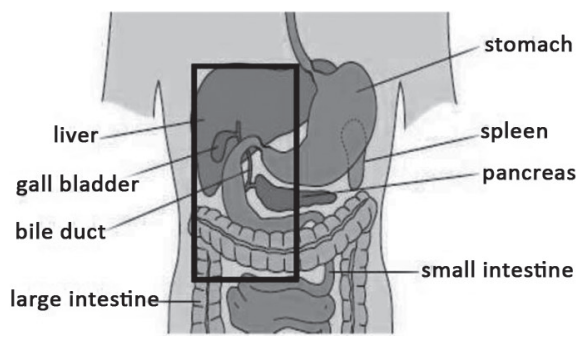

B

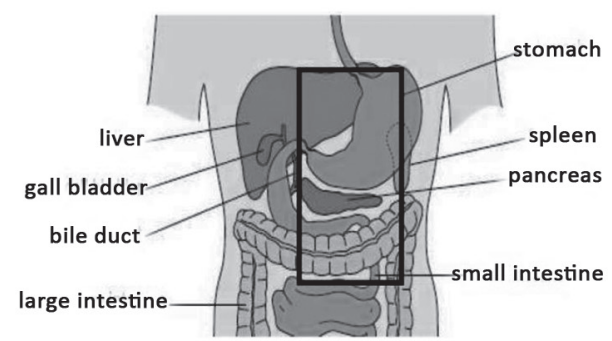

D

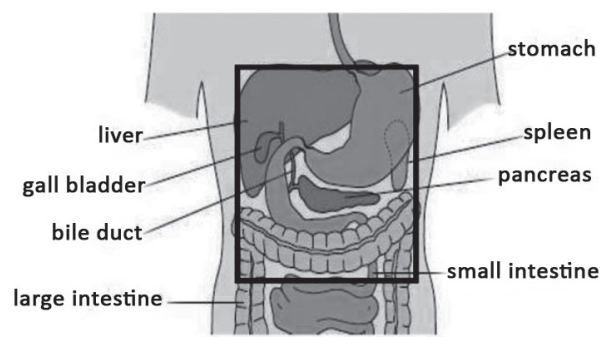

\section{Figure 1}

Legend: The black box indicates a schematic interpretation of the radiation field. $\mathbf{A}=$ Part of pancreas (i.e. head and part of tail) and part of liver in radiation field. $\mathbf{B}=$ Total pancreas (i.e. head and tail) and part of liver in radiation field. $\mathbf{C}=$ Part of pancreas (i.e. head and part of tail) and total liver in radiation field. $\mathbf{D}=$ Total pancreas (i.e. head and tail) and total liver in radiation field

Categories: $\mathbf{B}+\mathbf{D}=$ Total pancreas in radiation field. $\mathbf{A}+\mathbf{C}=$ Part of pancreas in radiation field. $\mathbf{C}+\mathbf{D}=$ Total liver in radiation field. $\mathbf{A}+\mathbf{B}=$ Part of liver in radiation field.

\section{Laboratory measurements}

Fasting blood samples were obtained from an intravenous-cannula before 10:00 a.m. Serum values of triglyceride $(\mathrm{mmol} / \mathrm{l}), \mathrm{HDL}-\mathrm{C}(\mathrm{mmol} / \mathrm{l}), \mathrm{LDL}-\mathrm{C}(\mathrm{mmol} / \mathrm{l})$, glucose $(\mathrm{mmol} / \mathrm{l})$ and free fatty acids (FFA) (mmol/l) were measured using an enzymatic in vitro assay (Roche Diagnostics, Mannheim, Germany). The intra- and interassay coefficients of variation (CV) were $<2 \%$ and $<3 \%$ for triglycerides, $<1 \%$ and $<2 \%$ for HDL-C, $<1 \%$ and $<2 \%$ for LDL-C, $<2 \%$ and $<2 \%$ for glucose and 
$<2 \%$ and $<3 \%$ for FFA. Serum insulin levels ( $\mathrm{pmol} / \mathrm{l}$ ) were measured using a chemi-luminescencebased immunoassay (Immulite 2000, Siemens DPC, Los Angeles, CA, USA). Intra- and interassay $\mathrm{CV}$ were $<6 \%$ and $<7 \%$ for insulin. Homeostatic model assessment (HOMA), that quantifies insulin resistance and beta cell function, was calculated. ${ }^{25}$

\section{Statistics}

Statistical analyses were performed with the Statistical Package for Social Sciences (SPSS 17.0, Chicago, IL, USA). P-values < 0.05 (two-tailed) were considered statistically significant. Independent Sample T-tests were used to compare results in survivors and controls and in subgroups. Metabolic syndrome and its separate components were evaluated with Chi-squared tests, all subjects were included. When assessing the metabolic syndrome, information can be lost as outcomes are dichotomized. Metabolic syndrome is designed for use in clinical settings but for research purposes the use of continuous variables provides more information. Therefore multiple linear regression analyses were performed with the outcome parameters glucose, triglycerides, HDL-C, waist circumference, systolic and diastolic blood pressure (components of metabolic syndrome) and insulin, HOMA, LDL-C, FFA (parameters considered associated with metabolic syndrome) as dependent variables. Glucose, insulin, HOMA and triglycerides levels were normally distributed after log-transformation and were expressed in percentages. Logistic regression analysis was performed with the outcome parameter metabolic syndrome (yes/no) as dependent variable and ordinal regression analysis was performed with the number of components of metabolic syndrome as dependent variable. For multiple linear regression analyses subjects treated for diabetes were excluded when evaluating glucose, insulin and FFA; subjects treated for hypertension were excluded when evaluating blood pressure; subjects treated for dyslipidemia were excluded when evaluating triglycerides, HDL-C and LDL-C. All models were corrected for age and sex. When the P-value was $<0.200$ for the possible confounders educational level, BMI, smoking and physical activity, these confounders were kept in the subsequent models. In model 1 the effects of the diagnoses (dummy variables) were evaluated. In model $2-4$ the effects of chemotherapy, nephrectomy, adrenalectomy and abdominal radiotherapy (dummy variables) were evaluated. Controls were indicated as not treated for treatment variables. Strategies of the multiple regression analyses are described in the legends of Table 3. We included SDS for total percentage fat $\geq 2$ as a surrogate adiposity component for metabolic syndrome. Frequency of SDS $\geq 2$ for total percentage fat was compared between survivors treated with and without abdominal radiotherapy. 


\section{Results}

\section{Survivors and controls}

In this study we included 164 subjects, i.e. 103 adult long-term survivors of nephroblastoma and neuroblastoma and 61 control subjects. Out of 88 adult long-term survivors of nephroblastoma who were alive and living in the Netherlands, 67 (28 females) participated (six were lost to followup, two not able to visit the outpatient clinic at the appointed time and 13 refused). Median age was 30.2 (range 18.8 - 50.8) years and median follow-up time was $26.2(6.4-48.9$ ) years. Survivors of neuroblastoma stage $4 \mathrm{~s}$ who had not received any previous treatment were excluded from this study. Out of 55 remaining neuroblastoma survivors, 36 (21 females) participated (five were lost to follow-up, three were pregnant at the time of the study and six refused). Median age was 29.6 (20.4 - 46.2) years and median follow-up time was $27.8(15.0-44.4)$ years. Survivors who did not participate were not different from participating survivors with respect to baseline characteristics (data not shown).

Fifty-eight percent of survivors had a sibling or friend willing to participate, resulting in 61 participating control subjects (28 females). Thirty-seven control subjects were siblings and 24 were partners/friends. Main reason for control-subjects not to participate was that they had to take a day off work. Baseline and treatment characteristics are shown in Table 1.

\section{Insulin resistance}

After adjusting for confounders, glucose levels were lower after adrenalectomy $(\beta=-8.7 \%$, $\mathrm{P}=0.001$ ) (Table 3). Five survivors were treated for type 2 diabetes at time of the study. Four out of five survivors who received irradiation to the total pancreas versus $1 / 5$ of the survivors who received irradiation to part of the pancreas had diabetes. Survivors who received radiotherapy to the total pancreas had higher glucose levels than controls $(\beta=10.5, P=0.002)$. Insulin levels were not determined by diagnosis, type of chemotherapy, surgery or radiotherapy field (Table 3 ). After adjusting for age, sex, educational level, smoking and BMI, HOMA was not significantly different between non-diabetic survivors of nephroblastoma ( $\beta=16.4 \%, P=0.075)$ and neuroblastoma $(\beta-4.0 \%, P=0.70)$ and controls. HOMA was not affected by nephrectomy, adrenalectomy or chemotherapy. Survivors who received radiotherapy to the total pancreas area had higher HOMA levels than controls $(\beta=27.0 \%, P=0.034)$, in contrast to survivors who received radiotherapy to part of the pancreas $(\beta=3.7 \%, P=0.78)$.

\section{Blood pressure}

Thirty-nine percent of nephroblastoma survivors had arterial hypertension or treatment for hypertension versus $29 \%$ of neuroblastoma survivors and $14 \%$ of controls (Table 2 ). After adjusting for age, sex, BMI and smoking, both nephroblastoma and neuroblastoma survivors had higher systolic $(\beta=5.6, P=0.007$ and $\beta=7.1, P=0.003$, respectively) and diastolic $(\beta=4.8, P=0.001$ and $\beta=6.0, P=0.001$, respectively) blood pressure levels than controls. Abdominal radiotherapy was 
associated with higher systolic $(\beta=6.1, P=0.021)$ and diastolic $(\beta=4.9, P=0.011)$ blood pressure. Chemotherapy only was associated with higher systolic blood pressure $(\beta=4.9, P=0.041)$.

Table 1. Baseline characteristics of study participants.

\begin{tabular}{|c|c|c|c|c|c|}
\hline & & Nephroblastoma & & Neuroblastoma & Controls \\
\hline$N$ & & 67 & & 36 & 61 \\
\hline Male/female & & $39 / 28$ & & $15 / 21$ & $33 / 28$ \\
\hline Age at follow-up (yrs) & & $30.2(18.8-50.8)$ & & $29.6(20.4-46.2)$ & $31.8(18.0-61.8)$ \\
\hline Age at diagnosis (yrs) & & $3.3(0.0-12.7)$ & & $0.8(0.0-11.7)$ & n.a. \\
\hline Follow-up time ${ }^{a}(y r s)$ & & $26.2(6.4-48.9)$ & & $27.8(15.0-44.4)$ & n.a. \\
\hline BMI $\left(\mathrm{kg} / \mathrm{m}^{2}\right)^{\mathrm{b}}$ & & $24.6(21.6-27.1)$ & & $24.1(20.8-26.0)$ & $24.2(22.1-27.4)$ \\
\hline Physical activity score ${ }^{\mathrm{b}}$ & & $\begin{array}{c}8280 \\
(6660-12000)\end{array}$ & & $\begin{array}{c}7130 \\
(5843-9005)\end{array}$ & $\begin{array}{c}8330 \\
(6570-11370)\end{array}$ \\
\hline Non smoker & & $66 \%$ & & $50 \%$ & $53 \%$ \\
\hline Former smoker & & $12 \%$ & & $19 \%$ & $16 \%$ \\
\hline Smoker & & $22 \%$ & & $31 \%$ & $31 \%$ \\
\hline Low educational level & & $21 \%$ & & $22 \%$ & $16 \%$ \\
\hline Medium educational level & & $36 \%$ & & $33 \%$ & $48 \%$ \\
\hline High educational level & & $43 \%$ & & $45 \%$ & $36 \%$ \\
\hline (Partial) adrenalectomy (N) & & $33 / 67$ & & $13 / 36$ & n.a. \\
\hline (Partial) nephrectomy (N) & & $67 / 67$ & & $7 / 36$ & n.a. \\
\hline $\begin{array}{l}\text { Radiotherapy }(\mathrm{N}) \\
\text { abdomen } \\
\text { thorax } \\
\text { neck } \\
\text { spine }\end{array}$ & & $\begin{array}{c}35 \\
0 \\
0 \\
0\end{array}$ & & $\begin{array}{l}7 \\
2 \\
1 \\
1\end{array}$ & n.a. \\
\hline Cumulative dose radiotherapy (gy) & & $20(15-40)$ & & $20(10-30)$ & n.a. \\
\hline \multirow[t]{2}{*}{ Chemotherapy (N) } & & $59 / 67$ & & $31 / 36$ & n.a. \\
\hline & $\mathbf{N}$ & TCD & $\mathbf{N}$ & TCD & \\
\hline Vincristin $\left(\mathrm{mg} / \mathrm{m}^{2}\right)$ & 51 & $22.0(6.0-93.0)$ & 16 & $22.8(5.0-65.0)$ & n.a. \\
\hline actinomycin $\mathrm{D}\left(\mathrm{mg} / \mathrm{m}^{2}\right)$ & 48 & $10.9(0.1-24.8)$ & 0 & 0 & n.a. \\
\hline anthracyclines $\left(\mathrm{mg} / \mathrm{m}^{2}\right)$ & 18 & $250(100-450)$ & 12 & $210(100-315)$ & n.a. \\
\hline cyclophosphamide $\left(\mathrm{mg} / \mathrm{m}^{2}\right)$ & 2 & $3825(250-7400)$ & 29 & $7350(3150-45990)$ & n.a. \\
\hline cisplatin $\left(\mathrm{mg} / \mathrm{m}^{2}\right)$ & 0 & 0 & 6 & $450(270-630)$ & n.a. \\
\hline teniposide $\left(\mathrm{mg} / \mathrm{m}^{2}\right)$ & 0 & 0 & 6 & $500(300-700)$ & n.a. \\
\hline dacarbazine $\left(\mathrm{mg} / \mathrm{m}^{2}\right)$ & 2 & $14.7(13.5-15.8)$ & 0 & 0 & n.a. \\
\hline iphosphamide $\left(\mathrm{mg} / \mathrm{m}^{2}\right)$ & 2 & $33000(30000-36000)$ & 0 & 0 & n.a. \\
\hline
\end{tabular}

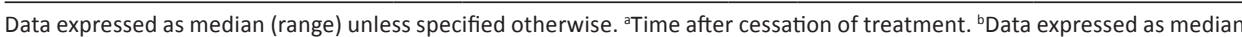
(interquartile range). $\mathbf{N} . \mathbf{A} .=$ not applicable; $\mathbf{G y}=$ gray; $\mathbf{T C D}=$ total cumulative dose; $\mathbf{B M I}=$ body mass index. 
Table 2. Prevalence of components of the metabolic syndrome in nephroblastoma and neuroblastoma survivors compared with controls.

\begin{tabular}{|c|c|c|c|c|c|}
\hline & $\begin{array}{c}\text { Controls } \\
(\mathrm{N}=61)\end{array}$ & $\begin{array}{l}\text { Nephroblastoma } \\
\quad(\mathrm{N}=67)\end{array}$ & P-value $^{a}$ & $\begin{array}{l}\text { Neuroblastoma } \\
(\mathrm{N}=36)\end{array}$ & $P_{\text {-value }}{ }^{b}$ \\
\hline Fasting glucose ${ }^{c}$ & $14 \%$ & $22 \%$ & 0.27 & $20 \%$ & 0.49 \\
\hline Hypertension $^{c}$ & $14 \%$ & $39 \%$ & 0.002 & $29 \%$ & 0.081 \\
\hline Low HDL-C & $20 \%$ & $24 \%$ & 0.66 & $29 \%$ & 0.39 \\
\hline High Triglycerides $^{c}$ & $11 \%$ & $27 \%$ & 0.031 & $18 \%$ & 0.35 \\
\hline High LDL-C & $21 \%$ & $31 \%$ & 0.20 & $31 \%$ & 0.32 \\
\hline High Waist ${ }^{d}$ & $10 \%$ & $6 \%$ & 0.44 & $12 \%$ & 0.73 \\
\hline High \% total body fat & n.a. & $15 \%$ & n.a. & $19 \%$ & n.a. \\
\hline
\end{tabular}

All subjects are included. Frequency of fasting glucose does not include one survivor with diabetes type 1. ${ }^{\text {Nephroblastoma }}$ survivors compared with controls ${ }^{b}$ Neuroblastoma survivors compared with controls (Chi-squared test) ${ }^{\mathrm{C} O r}$ treatment ${ }^{d}$ Waist circumference is imprecise in irradiated survivors. HDL-C = high-density lipoprotein-cholesterol; LDL-C = low-density lipoprotein-cholesterol

\section{Dyslipidemia}

High triglyceride levels or treatment for dyslipidemia were registered in $27 \%$ of nephroblastoma survivors, $18 \%$ of neuroblastoma survivors and in $11 \%$ of controls (Table 2 ). After adjusting for confounders, nephroblastoma survivors had higher triglyceride levels $(\beta=30.2, P=0.001)$, which seemed to be determined by abdominal radiotherapy $(\beta=33.8, P=0.005)$ and chemotherapy only ( $\beta=28.3, P=0.009$ ) (Table 3). Survivors who received radiotherapy to the total pancreas had significantly higher triglyceride levels $(\beta=44.8 \%, P=0.002)$ than controls, in contrast to survivors who received radiotherapy to part of the pancreas $(\beta=19.7 \%, P=0.159)$ (Table 3 ).

Diagnosis, treatment components and radiotherapy field did not affect the HDL-C levels (Table 3). After adjusting for confounders, LDL-C levels were higher in neuroblastoma survivors ( $\beta=0.44$, $P=0.004)$. Chemotherapy only $(\beta=0.45, P=0.003)$ and abdominal radiotherapy $(\beta=0.32, P=0.049)$ were positively associated with LDL-C levels, whereas adrenalectomy was negatively associated with LDL-C levels $(\beta-0.40, P=0.014)$. Survivors who received radiotherapy to the total liver had higher LDL-C levels $(\beta=0.35, P=0.044)$ than controls (Table 3 ).

After adjusting for confounders, abdominal radiotherapy was positively associated with higher FFA levels $(\beta=0.16, P<0.001)$, whereas chemotherapy only was negatively associated with FFA levels $(\beta=-0.13, P=0.005)$. Survivors who received radiotherapy to the total pancreas had significantly higher FFA levels than controls $(\beta=0.23, P<0.001)$, in contrast to survivors who received radiotherapy to only part of the pancreas $(\beta=0.09, P=0.121)$ (Table 3$)$. 


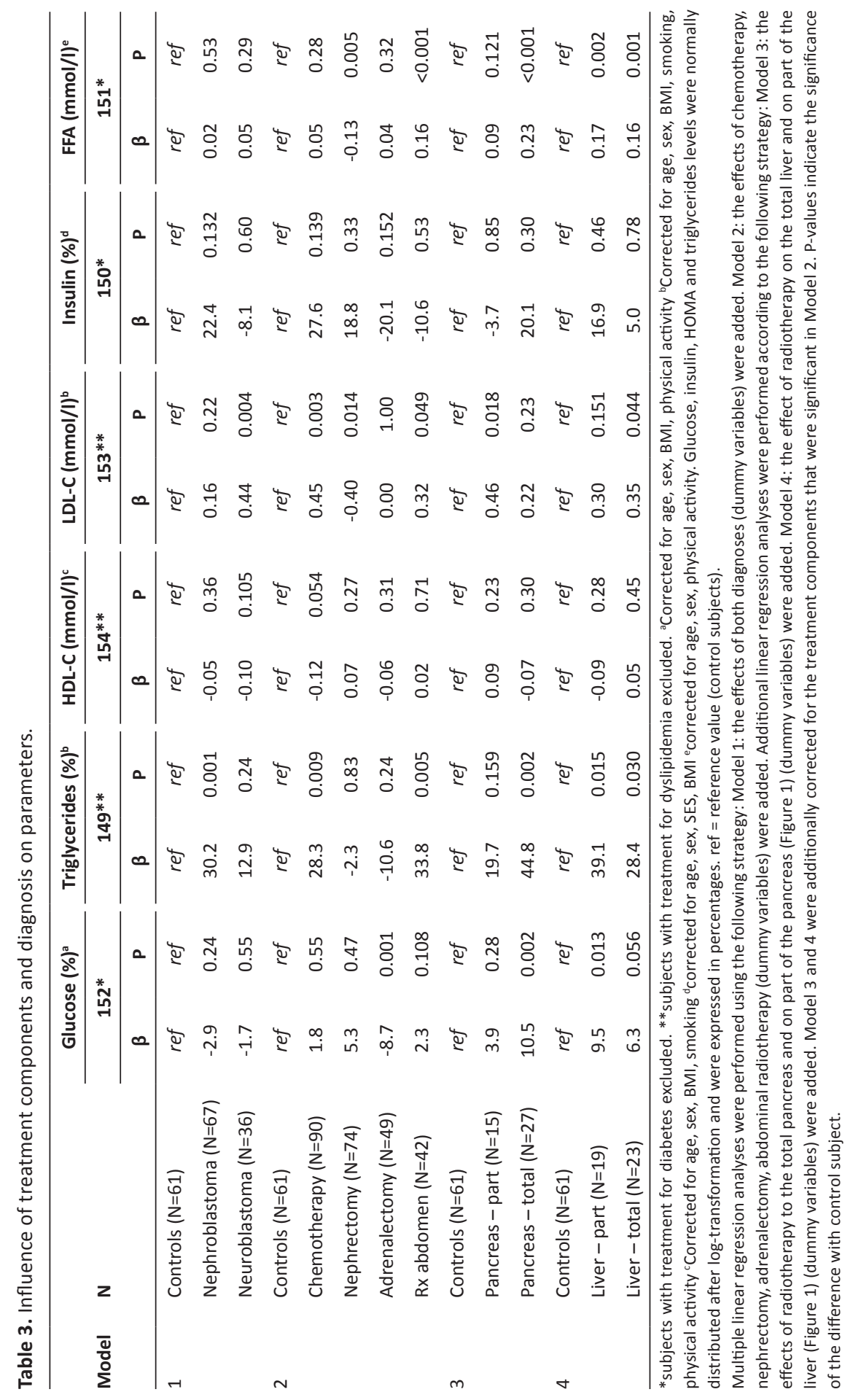




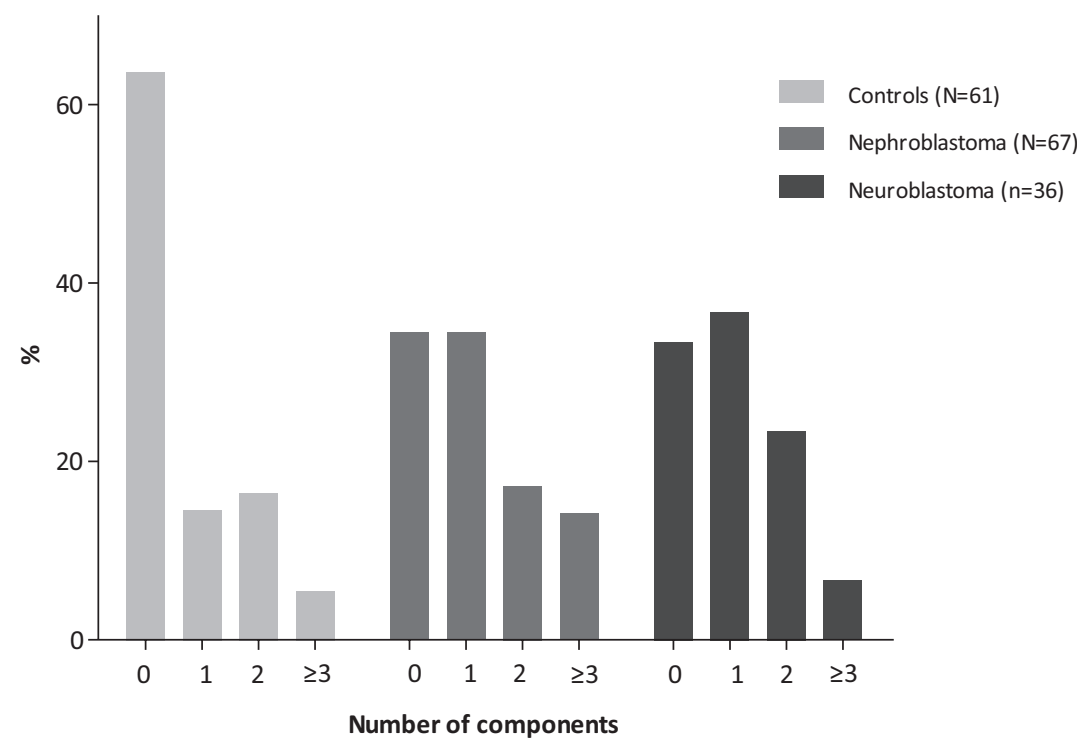

Figure 2. Components of the metabolic syndrome in nephroblastoma and neuroblastoma survivors. Frequency of metabolic syndrome determined according to the definition of the NCEP.

\section{Adiposity measurements}

After correction for age and sex, nephroblastoma survivors had a smaller waist circumference $(\beta=-4.7 \mathrm{~cm}, P<0.001)$ than controls. After additional correction for $B M I$, low waist circumference was associated with abdominal radiotherapy $(\beta=-5.6, P<0.001)$ but not with diagnosis or surgery. It is conceivable that this abnormal waist circumference is determined by anatomical derangements due to abdominal irradiation. Therefore, suggorate markers for adiposity were included in the analyses. After adjusting for age, sex and BMI, abdominal radiotherapy was associated with a lower waist/hip ratio $(\beta-0.05, P<0.001)$ and with a lower abdominal fat/total body fat ratio $(\beta=-1.9, P<0.001)$ but not with total body fat percentage $(\beta=1.4, P=0.29)$.

High waist circumference was present in $14 \%$ of the survivors treated without abdominal radiotherapy and in $2 \%$ of the survivors treated with abdominal radiotherapy $(P=0.020)$. Using total body fat (as measured by DXA-scan) as a surrogate marker for adiposity, $9 \%$ of the survivors treated without abdominal radiotherapy was categorized as adipose versus $28 \%$ of the survivors treated with abdominal radiotherapy $(P=0.018)$.

\section{Metabolic syndrome}

Prevalence of metabolic syndrome according to the NCEP criteria was not significantly different between nephroblastoma and neuroblastoma survivors and controls (Figure 2). After adjusting for age, sex, educational level and BMI, odds ratios (OR) for the frequency of metabolic syndrome as 
compared with controls were still not significant (ORs for nephroblastoma survivors 4.3, $P=0.093$, OR for neuroblastoma survivors $2.7, P=0.38$ ). However, $O R$ for the number of components of the metabolic syndrome were $5.2(P<0.001)$ in nephroblastoma and 6.5 in neuroblastoma $(P<0.001)$ survivors.

Subsequently, total fat percentage as measured by DXA scan was used as a surrogate marker for the adiposity component of metabolic syndrome. Metabolic syndrome was almost three times more frequent in abdominally irradiated survivors (28\%) than in non-irradiated survivors $(9 \%, \mathrm{P}=0.018)$ (Figure 3).

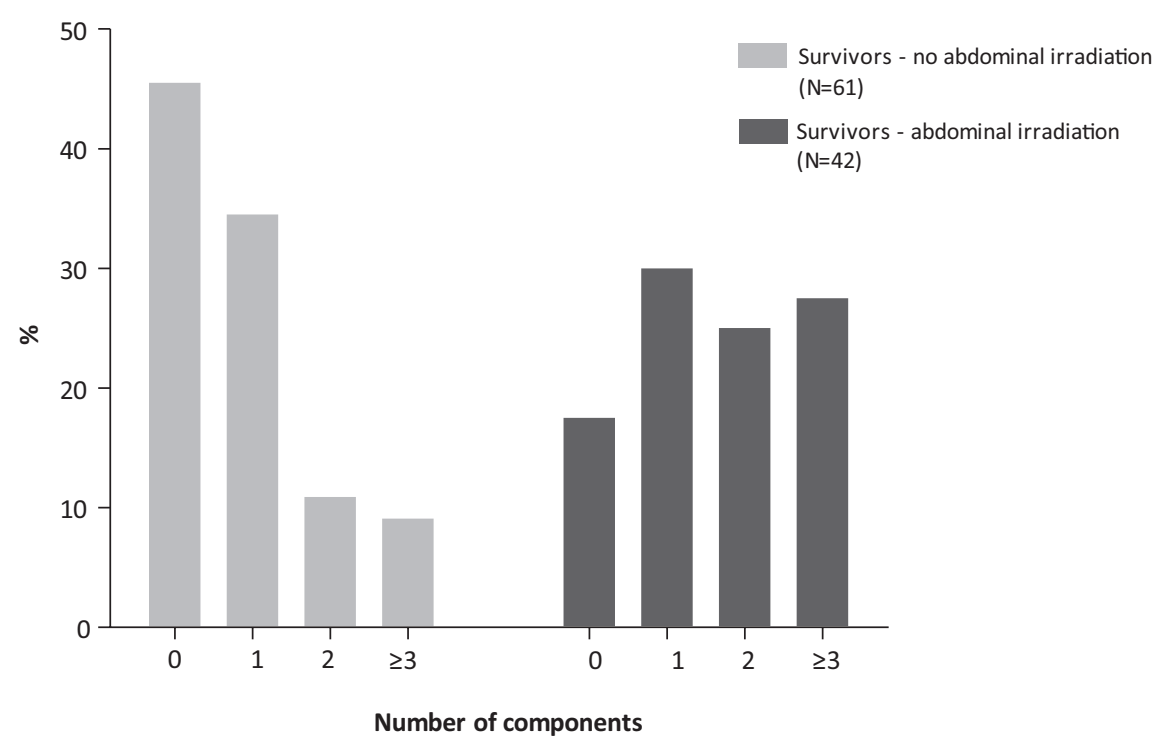

Figure 3. Components of the metabolic syndrome in survivors treated with and without abdominal irradiation.

Frequency of the metabolic syndrome determined according to the definition of NCEP but instead of waist circumference, total percentage fat was used as an alternative marker for adiposity. Prevalence of metabolic syndrome ( $\geq 3$ components) in abdominally irradiated survivors is significantly higher than in controls $(P=0.018)$ (Chi-squared test).

\section{Discussion}

In the present study we show that abdominal irradiation is the main determinant of metabolic syndrome in very long-term survivors of nephroblastoma and neuroblastoma, which was mainly due to hypertension, adiposity and dyslipidemia. Metabolic syndrome occurred three times more often in abdominally irradiated survivors, which is significant, especially when the relatively young age of the survivors and the fact that the incidence of cardiovascular diseases increases with age is taken into account. ${ }^{26}$ 
In the current study, hypertension and dyslipidemia in nephroblastoma and neuroblastoma survivors were mainly determined by previously administered abdominal irradiation. This is consistent with findings of the Childhood Cancer Survivor Study who found exposure to total body irradiation or abdominal plus chest irradiation to be associated with dyslipidemia, arterial hypertension and diabetes. ${ }^{27}$ In addition, increased prevalence of hypertension after abdominal irradiation was observed in a study including 62 nephroblastoma survivors ${ }^{28}$ and increased prevalence of cardiovascular events after abdominal irradiation was reported in a study including 185 nephroblastoma survivors. ${ }^{29}$ We hypothesized that the effect of abdominal radiotherapy on development of (components of the) metabolic syndrome may be due to radiation induced damage on individual organs. Therefore, we categorized patients according to administered radiation field. Although the pancreas has always been considered as relatively insensitive to radiation, ${ }^{30-31}$ we show that radiotherapy involving both head and tail of the pancreas influenced the occurrence of dyslipidemia and insulin resistance significantly. This is probably due to radiation induced apoptosis of pancreatic beta cells, and consequently to decreased insulin production, the thereby induced hyperglycemia, elevated FFA levels and hypertriglyceridemia and insulin resistance. We also show that abdominal irradiation involving the complete liver influences LDL-C levels, in contrast to irradiation involving only part of the liver. These results indicate that non-irradiated liver and non-irradiated pancreas fields may have the capacity to compensate for impaired liver and pancreas function of the irradiated fields. Although we were only able to categorize radiation fields, these single centre study results emphasize the need for studies in larger cohorts of childhood cancer survivors involving detailed radiation dosimetry, as this might help to unravel the mechanism behind the development of metabolic syndrome.

This is the first study that acknowledges the need for surrogate markers for adiposity in survivors after abdominal irradiation with a deformed abdominal area. We show that the measurement of total body fat probably provides a more reliable measurement of adiposity than waist circumference in abdominally irradiated survivors. It should be considered that abdominal fat is a better predictor of visceral fat than total body fat, however this needs the use of a computerised tomography (CT) or Magnetic Resonance Imaging (MRI) scan, which are expensive, time-consuming and undesirable in childhood cancer survivors who have often already been exposed to teratogenic treatments. Clinicians and researchers however need to be aware of the underestimation of adiposity and metabolic syndrome and, subsequently, the risk for cardiovascular diseases, when using waist circumference as a parameter in this specific subgroup.

Besides abdominal radiotherapy, we observed chemotherapy only to be associated with higher systolic blood pressure and dyslipidemia. Although the exact mechanism remains to be elucidated, it might well be that chemotherapeutic agents damage the vascular endothelium, resulting in vascular alterations. In the current study we found nephrectomy to be associated with lower LDL-C and FFA levels, whereas adrenalectomy was associated with lower glucose levels. Both types of surgery thus do not seem to increase the risk for development of 
metabolic syndrome. We previously, however, showed that adrenalectomized survivors have a higher setpoint of the hypothalamic pituitary axis. This resulted in high basal cortisol and adrenocorticotrophin hormone (ACTH) levels which were associated with insulin resistance and dyslipidemia. ${ }^{32}$ This indicates that although there might not be a direct effect of nephrectomy and adrenelectomy on metabolic syndrome, indirect pathways might still increase the risk for metabolic syndrome. Detailed measures of renal and adrenal function are therefore needed to determine the effects of nephrectomy and adrenalectomy on metabolic syndrome.

Although our study was limited by small sample sizes due to therapy subgroups, we are the first to report on metabolic syndrome and associated measures in survivors of nephroblastoma and neuroblastoma. This study provides information at one timepoint and in the future measurements of these survivors in time, including also cardiovascular endpoints, will be of importance for understanding the risk factors for subgroups of childhood cancer survivors.

In conclusion, our study demonstrated that nephroblastoma and neuroblastoma survivors are at increased risk for developing (components of) metabolic syndrome, and that abdominal radiotherapy is the main determinant of its occurrence. Surrogate markers for adiposity, such as total body fat, should be included in future studies in order to get insight in the true frequency of metabolic syndrome after abdominal irradiation. Prospective studies in large cohorts including radiation dosimetry are necessary to be able to unravel the underlying mechanisms behind the increased risk for developing components of metabolic syndrome after abdominal radiotherapy. 


\section{References}

1. Bernstein ML, Leclerc JM, Bunin G, Brisson L, Robison L, Shuster J, et al. A population-based study of neuroblastoma incidence, survival, and mortality in North America. J Clin Oncol 1992;10(2):323-9.

2. Bernstein L, Linet $M$, Smith $M$, Olshan AF. Cancer incidence and survival among children and adolescents: United States SEER Program 1975-1995, SEER Program. National Cancer Institute 1999 1999:p.79-90.

3. van Waas M, Neggers SJ, van der Lelij AJ, Pieters R, van den Heuvel-Eibrink MM. The metabolic syndrome in adult survivors of childhood cancer, a review. J Pediatr Hematol Oncol 2010;32(3):171-9.

4. van Waas M, Neggers SJ, Pieters R, van den Heuvel-Eibrink MM. Components of the metabolic syndrome in 500 adult long-term survivors of childhood cancer. Ann Oncol 2010;21(5):1121-6.

5. van Waas M, Neggers SJ, Te Winkel ML, Beishuizen A, Pieters R, van den Heuvel-Eibrink MM. Endocrine late sequelae in long-term survivors of childhood non-Hodgkin lymphoma. Ann Oncol 2011 June;23(6):1626-32.

6. van Beek RD, van den Heuvel-Eibrink MM, Hakvoort-Cammel FG, van den Bos C, van der Pal HJ, Krenning EP, et al. Bone mineral density, growth, and thyroid function in long-term survivors of pediatric Hodgkin's lymphoma treated with chemotherapy only. J Clin Endocrinol Metab 2009;94(6):1904-9.

7. Blijdorp K, van den Heuvel-Eibrink M, Pieters R, Boot A, Sluimer J, van der Lelij AJ, et al. The limited screening value of insulin-like growth factor-i as a marker for alterations in body composition in very long-term adult survivors of childhood cancer. Pediatr Blood Cancer 2011 Dec 11. doi: 10.1002/ pbc. 24015.

8. Grundy SM, Becker D, Clark LT, Cooper RS, Denke MA, Howard J, et al. Executive Summary of The Third Report of The National Cholesterol Education Program (NCEP) Expert Panel on Detection, Evaluation, And Treatment of High Blood Cholesterol In Adults (Adult Treatment Panel III). JAMA 2001;285(19):2486-97.

9. Reaven GM. Banting lecture 1988. Role of insulin resistance in human disease. Diabetes 1988;37(12):1595-607.

10. Kershaw EE, Flier JS. Adipose tissue as an endocrine organ. J Clin Endocrinol Metab 2004;89(6):254856.

11. Yusuf S, Hawken S, Ounpuu S, Dans T, Avezum A, Lanas F, et al. Effect of potentially modifiable risk factors associated with myocardial infarction in 52 countries (the INTERHEART study): case-control study. Lancet 2004;364(9438):937-52.

12. Montague CT, O’Rahilly S. The perils of portliness: causes and consequences of visceral adiposity. Diabetes 2000;49(6):883-8.

13. Kern PA, Ranganathan S, Li C, Wood L, Ranganathan G. Adipose tissue tumor necrosis factor and interleukin- 6 expression in human obesity and insulin resistance. Am J Physiol Endocrinol Metab 2001;280(5):E745-51.

14. Marette A. Molecular mechanisms of inflammation in obesity-linked insulin resistance. Int J Obes Relat Metab Disord 2003;27 Suppl 3:S46-8.

15. Laverdiere C, Cheung NK, Kushner BH, Kramer K, Modak S, LaQuaglia MP, et al. Long-term complications in survivors of advanced stage neuroblastoma. Pediatr Blood Cancer 2005;45(3):324-32.

16. Sasso G, Greco N, Murino P, Sasso FS. Late toxicity in Wilms tumor patients treated with radiotherapy at 15 years of median follow-up. J Pediatr Hematol Oncol 2010;32(7):e264-7.

17. Wright KD, Green DM, Daw NC. Late effects of treatment for wilms tumor. Pediatr Hematol Oncol 2009;26(6):407-13.

18. Assembly WMAG. Declaration of Helsinki - Sixth revision. 2008.

19. Wendel-Vos GC, Schuit AJ, Saris WH, Kromhout D. Reproducibility and relative validity of the short questionnaire to assess health-enhancing physical activity. J Clin Epidemiol 2003;56(12):1163-9.

20. Khosla T, Lowe CR. Indices of obesity derived from body weight and height. Br J Prev Soc Med $1967 ; 21(3): 122-8$. 
21. WHO. Waist circumference and Waist-Hip Ratio: Report of a WHO Expert Consultation. Geneva, 8-11 December 2008. 2008.

22. Expert Panel on Detection E, Treatment of High Blood Cholesterol in A. Executive Summary of The Third Report of The National Cholesterol Education Program (NCEP) Expert Panel on Detection, Evaluation, And Treatment of High Blood Cholesterol In Adults (Adult Treatment Panel III). JAMA 2001;285(19):2486-97.

23. Pimenta N, Santa-Clara H, Fragoso IJ. Comparison of body composition and body fat distribution of patients following a cardiac rehabilitation program and sedentary patients. Rev Port Cardiol 2010;29(78):1163-80.

24. van der Sluis IM, de Ridder MA, Boot AM, Krenning EP, de Muinck Keizer-Schrama SM. Reference data for bone density and body composition measured with dual energy $x$ ray absorptiometry in white children and young adults. Arch Dis Child 2002;87(4):341-7.

25. Matthews DR, Hosker JP, Rudenski AS, Naylor BA, Treacher DF, Turner RC. Homeostasis model assessment: insulin resistance and beta-cell function from fasting plasma glucose and insulin concentrations in man. Diabetologia 1985;28(7):412-9.

26. Mackay J, Mensah G. The Atlas of Heart Disease and Stroke. World Health Organization 2004.

27. Meacham LR, Chow EJ, Ness KK, Kamdar KY, Chen Y, Yasui Y, et al. Cardiovascular risk factors in adult survivors of pediatric cancer--a report from the childhood cancer survivor study. Cancer Epidemiol Biomarkers Prev 2010;19(1):170-81.

28. Geenen MM, Bakker PJ, Kremer LC, Kastelein JJ, van Leeuwen FE. Increased prevalence of risk factors for cardiovascular disease in long-term survivors of acute lymphoblastic leukemia and Wilms tumor treated with radiotherapy. Pediatr Blood Cancer 2010;55(4):690-7.

29. van Dijk IW, Oldenburger F, Cardous-Ubbink MC, Geenen MM, Heinen RC, de Kraker J, et al. Evaluation of late adverse events in long-term wilms' tumor survivors. Int J Radiat Oncol Biol Phys 2010;78(2):3708.

30. Radiation CotBEol. Health Effects of Exposure to Low Levels of Ionizing Radiation. BEIR V Washington, DC: National Academy Press 1990.

31. Shimizu Y, Kato H, Schull WJ. Studies of the mortality of A-bomb survivors. 9. Mortality, 1950-1985: Part 2. Cancer mortality based on the recently revised doses (DS86). Radiat Res 1990;121(2):120-41.

32. Van Waas M, Pieters R, van Eck JP, Van Noesel MM, Van der Lelij AJ, De Jong FH, et al. Adrenal function in adult long-term survivors of nephroblastoma and neuroblastoma. Eur J Cancer 2012;48:1159-66. 


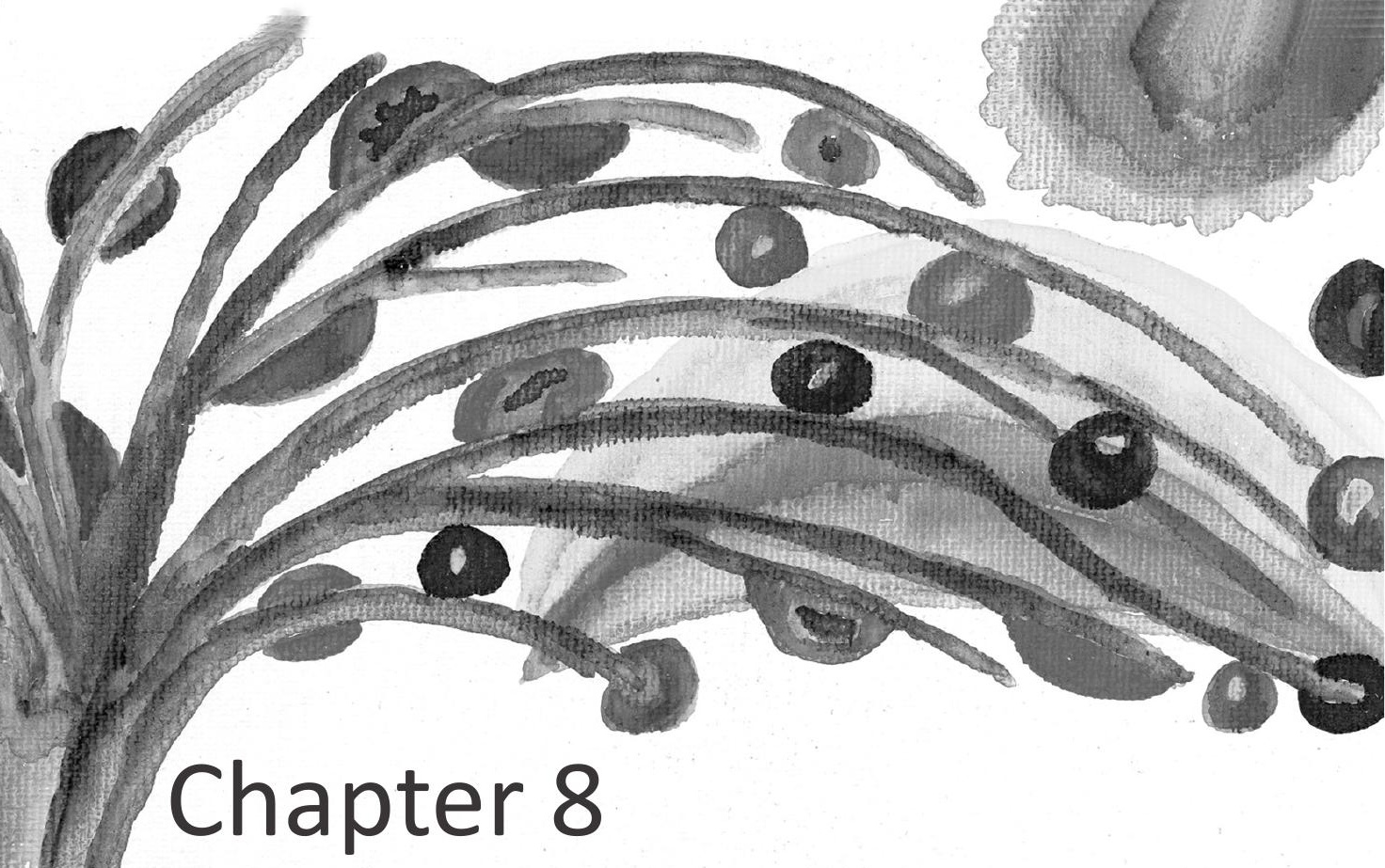

\title{
Cardiovascular risk factors in adult very
} long-term survivors of nephroblastoma and neuroblastoma

\author{
Marjolein van Waas' ${ }^{1}$, Sebastian J.C.M.M. ${ }^{1,2}$, Caspar W.N. Looman ${ }^{3}$, Hein Raat ${ }^{3}$, \\ Monique P.M. de Maat ${ }^{4}$, Robert de Jonge ${ }^{5}$, Patric J.D. Delhanty ${ }^{2}$, Martin Huisman², \\ Francesco U.S. Mattace-Raso ${ }^{6}$, Rob Pieters ${ }^{1}$, Marry M. van den Heuvel-Eibrink ${ }^{1}$
}

'Department of Pediatric Oncology/Hematology, Erasmus MC-Sophia Children's Hospital, Rotterdam, the Netherlands

${ }^{2}$ Department of Medicine, Section Endocrinology, Erasmus University Medical Centre, Rotterdam, the Netherlands

${ }^{3}$ Department of Public Health, Erasmus University Medical Centre, Rotterdam, the Netherlands ${ }^{4}$ Department of Hematology, Erasmus University Medical Centre, Rotterdam, the Netherlands ${ }^{5}$ Department of Clinical Chemistry, Erasmus University Medical Centre, Rotterdam, the Netherlands

${ }^{6}$ Department of Internal Medicine, Section of Geriatric Medicine, Erasmus University Medical Centre, Rotterdam, the Netherlands 


\section{Abstract}

Background: To date, reports on cardiovascular risk factors in nephroblastoma and neuroblastoma survivors are scarce and, so far, have focused on conventional and individual risk factors.

Patients and methods: In this prospective study 164 subjects (67 nephroblastoma survivors (28 females) and 36 neuroblastoma survivors ( 21 females), and 61 cross-sectionally recruited control subjects (28 females)) participated. Fasting blood samples were taken and a variety of biomarkers was assessed. Principal component analysis was used to identify underlying combinations of biomarkers. Pulse pressure, pulse pressure amplification, pulse wave velocity, intima media thickness and central blood pressure were assessed.

Results: Median age in survivors was 30 (18 - 51) years and median follow-up time was 27 $(6-49)$ years. Median age of control subjects was $32(18-62)$ years. Nephroblastoma but not neuroblastoma survivors had lower pulse pressure amplification $(\beta=-0.22, P=0.008)$ and higher central systolic blood pressure $(\beta=7.90, P=0.008)$ than controls. Abdominal radiotherapy was associated with higher pulse wave velocity $(\beta=0.89, P=0.017)$ and central diastolic blood pressure $(\beta=5.96, P=0.006)$. Seven underlying principal components of biomarkers were identified. Abdominal radiotherapy was associated with the "Adverse lipids component" $(\beta=0.49, P=0.044)$ and with the "Adipocyte derived factors component" $(\beta=0.58, P=0.018)$. Nephrectomy was associated with impaired renal function ( $\beta=0.63, P=0.013)$.

Conclusions: Nephroblastoma and neuroblastoma survivors express several cardiovascular risk factors. Abdominal irradiation and nephrectomy seem to be the most important determinants of risk for cardiovascular diseases. As relatively young aged survivors already express metabolic and vascular alterations, the relevance of these findings for the future is high. 


\section{Introduction}

Five-year survival of childhood nephroblastoma and neuroblastoma has increased significantly over the past few decades and is currently 92\% and $\sim 55 \%$, respectively. ${ }^{1,2}$ Increased prevalence of late effects has been reported in nephroblastoma survivors treated with abdominal radiotherapy. ${ }^{3,4}$ Treatment in these patients may effect health later in life, but reports on late effects, especially on cardiovascular risk factors in neuroblastoma survivors are scarce. ${ }^{5}$ Both tumors are of embryonic origin, have a peak incidence $<5$ years of age and present predominantly in the abdomen. ${ }^{6}$ Treatments for nephroblastoma and neuroblastoma include surgery (nephrectomy and/or adrenalectomy), abdominal radiotherapy, anthracyclines and vincristin. Studying the incidence of cardiovascular diseases in childhood cancer survivors is difficult because subjects are too young to use myocardial infarctions and/or mortality as unbiased endpoints. Therefore surrogate markers like blood pressure, low density lipoprotein-cholesterol (LDL-C), smoking behaviour and body mass index are used to predict cardiovascular diseases in young adults. New markers have been identified, such as central blood pressure and measures of arterial stiffness and intima media thickness which provide functional and structural information of the blood vessels. ${ }^{7-9}$ Pulse pressure has been reported to be an independent predictor of coronary heart disease $\mathrm{e}^{10}$ and impaired carotid-brachial pressure amplification is associated with an increased risk for cardiovascular diseases. ${ }^{11}$

Proinflammatory and prothrombotic factors and less conventional markers such as homocystein, uric acid, adiponectin, and leptin have been associated with the metabolic syndrome and a higher risk for cardiovascular disease. ${ }^{12-16}$ According to a recent review, a combination of biomarkers reflecting different pathophysiologic pathways will be most useful in yielding incremental information above and beyond that gained by using traditional parameters. ${ }^{17}$ This hypothesis is supported by several studies that have identified underlying factors of variables that are associated with metabolic syndrome, diabetes and cardiovascular diseases. ${ }^{18-21}$

In this unique prospective study of adult survivors of nephroblastoma and neuroblastoma and a cross-sectionally recruited controlgroup, we studied vascular parameters and used principal component analysis to identify combinations of biomarkers, as predictors of risk for developing cardiovascular diseases. Furthermore, we assessed the influence of surgery, radiotherapy, and chemotherapy on vascular parameters and identified combinations of biomarkers.

\section{Patients and methods}

\section{Patients}

All long-term ( $\geq 5$ years after cessation of treatment) adult survivors of childhood nephroblastoma and neuroblastoma, treated between 1961 - 2004 in the Erasmus MC - Sophia Children's Hospital who visit the Late Effects outpatient clinic regularly were invited to participate in this 
study. Informed consent was obtained according to the Helsinki declaration ${ }^{22}$ and the study was approved by the local medical ethical committee (NTR 2814). Survivors were asked to invite potential control subjects such as siblings, friends or neighbours, preferably of the same sex and within an age range of five years of the survivor.

\section{Data collection}

Disease and treatment data were obtained from our local database. Data regarding (partial) nephrectomy and unilateral adrenalectomy were confirmed from the original surgical and pathological reports. Information regarding smoking status, educational level, statine and antihypertensive medication and diabetes was collected using a self-designed questionnaire. Smoking status was defined as non-smoker, former smoker or current smoker. Educational level was defined by the highest level of educational attainment as selected from three categories based on the Dutch educational system. Daily life physical activity was assessed by the Short Questionnaire to Assess Health-enhancing physical activity (SQUASH). ${ }^{23}$ Height was measured to the nearest millimeter using a Harpenden Stadiometer and weight was assessed with underwear only to the nearest $0.1 \mathrm{~kg}$ with a standard clinical balance. Body mass index (BMI) was calculated (weight $(\mathrm{kg})) /\left(\right.$ height $\left.(\mathrm{cm})^{2}\right)$. Systolic and diastolic blood pressure was measured with the subject in sitting position after an hour of rest on the right arm with the Dinamap ${ }^{\circledR}$ Procare and was defined as the mean of three measurements.

\section{Laboratory measurements}

Fasting blood samples were taken from an intravenous-cannula before 10:00 a.m.. The following biomarkers were assessed: Serum values of triglyceride ( $\mathrm{mmol} / \mathrm{l}), \mathrm{HDL}-\mathrm{C}(\mathrm{mmol} / \mathrm{l}), \mathrm{LDL}-\mathrm{C}(\mathrm{mmol} / \mathrm{l})$, glucose (mmol/l), free fatty acids (FFA) ( $\mathrm{mmol} / \mathrm{l})$, lipoprotein a (lp $(\mathrm{a}))(\mathrm{mmol} / \mathrm{l})$, uric acid $(\mathrm{mmol} / \mathrm{l})$, urea (mmol/l), insulin (pmol/l), apolipoprotein-A1 (apo-A1) $(\mathrm{g} / \mathrm{l})$ and apo-B $(\mathrm{g} / \mathrm{l})$, leptin (ng/ $\mathrm{ml})$, high molecular weight $(\mathrm{HMW})$ adiponectin $(\mu \mathrm{g} / \mathrm{ml})$, homocystein $(\mu \mathrm{mol} / \mathrm{l})$, antithrombin III activity (IU/ml), protein C activity (IU/ml), protein S activity $(\mathrm{IU} / \mathrm{ml})$, Lupus Anticoagulans diluted Russell's Viper Venom Time (LA dRVVT) (sec), fibrinogen (g/l), von Willebrandfactor antigen (vWf-ag) (U/ml), plasminogen activator inhibitor-1 (PAI-1) $(\mathrm{IU} / \mathrm{ml})$, cystatin C $(\mathrm{mg} / \mathrm{l})$ and highsensitivity $\mathrm{C}$ reactive protein ( $\mathrm{hsCRP}$ ) ( $\mathrm{mg} / \mathrm{l}$ ) were assessed. Details on assays are supplied in the supplementary text. Homeostatic model assessment (HOMA), that quantifies insulin resistance and beta cell function, was calculated. ${ }^{24}$ Protein $\mathrm{C}$ and $\mathrm{S}, \mathrm{vWf}, \mathrm{LAdRVV}$ and antithrombin were measured to exclude subjects with possible coagulation problems and were not reported as outcome variables. Modification of Diet in Renal Disease (MDRD) study equation was calculated and MDRD below $60 \mathrm{~mL} / \mathrm{min} / 1.73 \mathrm{~m}^{2}$ was considered abnormal. ${ }^{25,26} \mathrm{~N}$-terminal pro-brain natriuretic peptide (NT-pro-BNP) (pmol/l) was determined as a marker for heart function. ${ }^{27}$ 


\section{Cardiovascular studies}

Carotid intima-media thickness (IMT) and common carotid distensibility were assessed with the subjects in supine position, with the head tilted slightly to the contralateral side. The vessel wall motion of the right common carotid artery was measured by means of a duplex scanner (operating frequency $7.5 \mathrm{MHz}$, Pie Medical, Esaote, Maastricht, the Netherlands) connected to a vessel wall movement detector system (analyzed with ART.LAB, Pie Medical, Esaote, Maastricht, the Netherlands). After five minutes of rest, a region at $1.0 \mathrm{~cm}$ proximal to the origin of the bulb of the carotid artery was identified with the use of B-mode ultrasound. Real-time calculation of IMT and distensibility was performed over six independent heartbeats and presentation included mean values and standard deviation. Distensibility coefficient (DC) was calculated according to the following formula: ((2000*distensibility)/diameter)/(pulse pressure*133.22). ${ }^{28,29}$ Pulse wave velocity (PWV) was calculated with the participants in supine position. PWV was assessed with an automatic device (Complior; Artech Medical, Pantin, France) that measures the time delay between the rapid upstroke of the feet of simultaneously recorded pulse waves in the carotid and the femoral arteries. ${ }^{30}$ The distance between the recording sites in the carotid and the femoral arteries was measured. PWV was calculated as the ratio between the distance and the foot-tofoot time delay $(\mathrm{m} / \mathrm{s})$. In a reproducibility study, the intraclass correlation coefficient was $0.80 .{ }^{31}$

Central blood pressure was also measured with subjects in the supine position using the Sphygmocor (AtCor Medical, Sydney, Australia), which uses a tonometry probe manually applied by a trained user on the radial artery pulse on the wrist. It records the pulse waveform, which is calibrated against upper arm cuff blood pressure. A generalized transfer function is applied to the radial waveform to derive that in the aorta and calculate central blood pressure. ${ }^{32}$

Brachial pulse pressure ${ }^{10}$ and pulse pressure amplification (brachial pulse pressure/central pulse pressure $)^{11}$ were calculated. Cardiac function was routinely assessed by measuring the left ventricular shortening fraction (LVSF) with transthoracic echocardiography in survivors treated with anthracyclines using a Philips iE33 or Sonos 7500 system (Philips, Best, The Netherlands). LVSF below $20 \%$ was defined as heart failure. ${ }^{33}$

\section{Statistics}

Statistical analyses were performed with the Statistical Package for Social Sciences (SPSS 17.0, Chicago, IL, USA). P-values <0.05 (two-tailed) were considered statistically significant. Mann Whitney U-tests were used to compare results in survivors and controls. When assessing the metabolic syndrome information can be lost as outcomes are dichotomized. Multiple linear regression analyses with the vascular parameters as dependent variables were performed. Multiple linear regression analyses were performed. All models were corrected for age and sex. When the P-value was $<0.200$ for the possible confounders educational level, BMI, smoking, physical activity, MDRD and NT-pro-BNP, the confounders were kept in the subsequent models. Model 1 was used to analyze the effect of both diagnoses. Model 2 was used to analyze the effect of the treatment components nephrectomy, adrenalectomy, abdominal radiotherapy and was 
adjusted for cumulative doses of cyclophosphamide, actinomycin, anthracyclines and vincristin. Principal component analysis (PCA) was used to identify correlations between biomarkers and to be able to reduce multiple testing while using all outcome variables. PCA is a multivariate correlation method that is well suited for revealing underlying patterns or structure among variables showing high degrees of intercorrelation. In the case of metabolic risk variables, the method identifies latent variables (so called "principal components") that can be interpreted as underlying continuous risk factors that can be calculated as weighted means of the observed variables. Varimax rotation redefines the solution in such a way that observed variables correlate either very high or very low to the factors/latent variables, which helps the interpretation of the factors. PCA was performed on normalized observations, i.e. Z-scores. Before performing PCA, missing data were imputed according to the following strategy: when less than $5 \%$ of data were missing, the median value was imputed. When $>5 \%$ of data were missing, customized models were developed to predict the covariate from all of the other covariates. Accordingly, missing data were imputed with predicted values. ${ }^{34}$ Log transformations of skewed variables were used in PCA. Varimax rotation was used to identify the components. We analyzed components with an Eigenvalue (the variance in all variables which is accounted for by that component) larger than one ${ }^{35}$ and observed seven underlying components (Table 4). Component scores, which represent the subjects' predicted values for each component, were calculated using the component weights and the original variable values. Variables with component loadings $>0.4$ and $<-0.4$ were included. A name was given to each component in the text based on the characterizing components (Table 4). Subsequently, multiple linear regression analyses with the component scores as dependent variables were performed, according to the strategy mentioned in the legends of Table 5.

\section{Results}

\section{Survivors and controls}

In this study, we included 164 subjects, i.e. 103 adult long-term survivors of childhood nephroblastoma and neuroblastoma and 61 control subjects. Out of 88 adult long-term survivors of nephroblastoma, 67 (28 females) who were alive and living in the Netherlands participated (six lost to follow-up, two not able to visit the outpatient clinic at the appointed time, 13 refused). Survivors of neuroblastoma stage $4 \mathrm{~s}$ who did not receive surgery, radiotherapy or chemotherapy were excluded from this study. Out of 55 remaining neuroblastoma survivors, 36 (21 females) participated (five lost to follow-up, three were pregnant at the time of the study, six refused). Survivors who did not participate were not different from participating survivors with respect to baseline characteristics.

Sixty-one control subjects participated (28 females). Thirty-seven control subjects were siblings and 24 were partner/friend. The main reason for control-subjects not to participate was that they had to take a day off work. Baseline and treatment characteristics are shown in Table 1. 
Table 1. Baseline characteristics of study participants.

\begin{tabular}{|c|c|c|c|c|c|}
\hline & \multicolumn{2}{|r|}{ Nephroblastoma } & \multicolumn{2}{|r|}{ Neuroblastoma } & Controls \\
\hline $\mathrm{N}$ & \multicolumn{2}{|r|}{67} & \multicolumn{2}{|r|}{36} & 61 \\
\hline Male/female & \multicolumn{2}{|r|}{$39 / 28$} & & $15 / 21$ & $33 / 28$ \\
\hline Age at follow-up (yrs) & \multicolumn{2}{|r|}{$30.2(18.8-50.8)$} & & $29.6(20.4-46.2)$ & $31.8(18.0-61.8)$ \\
\hline Age at diagnosis (yrs) & \multicolumn{2}{|r|}{$3.3(0.0-12.7)$} & & $0.8(0.0-11.7)$ & n.a. \\
\hline Follow-up time ${ }^{a}(y r s)$ & \multicolumn{2}{|r|}{$26.2(6.4-48.9)$} & & $27.8(15.0-44.4)$ & n.a. \\
\hline $\mathrm{BMI}\left(\mathrm{kg} / \mathrm{m}^{2}\right)^{\mathrm{b}}$ & \multicolumn{2}{|r|}{$24.6(21.6-27.1)$} & & $24.1(20.8-26.0)$ & $24.2(22.1-27.4)$ \\
\hline Physical activity score ${ }^{b}$ & \multicolumn{2}{|r|}{$\begin{array}{c}8280 \\
(6660-12000)\end{array}$} & & $\begin{array}{c}7130 \\
(5843-9005)\end{array}$ & $\begin{array}{c}8330 \\
(6570-11370)\end{array}$ \\
\hline Non smoker & \multicolumn{2}{|r|}{$66 \%$} & & $50 \%$ & $53 \%$ \\
\hline Former smoker & \multicolumn{2}{|r|}{$12 \%$} & & $19 \%$ & $16 \%$ \\
\hline Smoker & \multicolumn{2}{|r|}{$22 \%$} & & $31 \%$ & $31 \%$ \\
\hline Low educational level & \multicolumn{2}{|r|}{$21 \%$} & & $22 \%$ & $16 \%$ \\
\hline Medium educational level & \multicolumn{2}{|r|}{$36 \%$} & & $33 \%$ & $48 \%$ \\
\hline High educational level & \multicolumn{2}{|r|}{$43 \%$} & & $45 \%$ & $36 \%$ \\
\hline (Partial) adrenalectomy $(\mathrm{N})$ & \multicolumn{2}{|r|}{$33 / 67$} & & $13 / 36$ & n.a. \\
\hline (Partial) nephrectomy (N) & \multicolumn{2}{|r|}{$67 / 67$} & \multicolumn{2}{|r|}{$7 / 36$} & n.a. \\
\hline \multicolumn{6}{|l|}{ Radiotherapy (N) } \\
\hline abdomen & \multicolumn{2}{|r|}{35} & & 7 & \multirow[t]{4}{*}{ n.a. } \\
\hline thorax & \multicolumn{2}{|r|}{0} & \multicolumn{2}{|r|}{2} & \\
\hline neck & \multicolumn{2}{|r|}{0} & \multicolumn{2}{|r|}{1} & \\
\hline spine & \multicolumn{2}{|r|}{0} & \multicolumn{2}{|r|}{1} & \\
\hline Cumulative dose radiotherapy (gy) & & $20(15-40)$ & & $20(10-30)$ & n.a. \\
\hline \multirow[t]{2}{*}{ Chemotherapy $(\mathrm{N})$} & \multicolumn{2}{|r|}{$59 / 67$} & & $31 / 36$ & n.a. \\
\hline & $\mathbf{N}$ & TCD & $\mathbf{N}$ & TCD & \\
\hline Vincristin $\left(\mathrm{mg} / \mathrm{m}^{2}\right)$ & 51 & $22.0(6.0-93.0)$ & 16 & $22.8(5.0-65.0)$ & n.a. \\
\hline actinomycin $\mathrm{D}\left(\mathrm{mg} / \mathrm{m}^{2}\right)$ & 48 & $10.9(0.1-24.8)$ & 0 & 0 & n.a. \\
\hline anthracyclines $\left(\mathrm{mg} / \mathrm{m}^{2}\right)$ & 18 & $250(100-450)$ & 12 & $210(100-315)$ & n.a. \\
\hline cyclophosphamide $\left(\mathrm{mg} / \mathrm{m}^{2}\right)$ & 2 & $3825(250-7400)$ & 29 & $7350(3150-45990)$ & n.a. \\
\hline cisplatin $\left(\mathrm{mg} / \mathrm{m}^{2}\right)$ & 0 & 0 & 6 & $450(270-630)$ & n.a. \\
\hline teniposide $\left(\mathrm{mg} / \mathrm{m}^{2}\right)$ & 0 & 0 & 6 & $500(300-700)$ & n.a. \\
\hline dacarbazine $\left(\mathrm{mg} / \mathrm{m}^{2}\right)$ & 2 & $14.7(13.5-15.8)$ & 0 & 0 & n.a. \\
\hline iphosphamide $\left(\mathrm{mg} / \mathrm{m}^{2}\right)$ & 2 & $33000(30000-36000)$ & 0 & 0 & n.a. \\
\hline
\end{tabular}

Data expressed as median (range) unless specified otherwise. ${ }^{a}$ Time after cessation of treatment. ${ }^{b}$ Data expressed as median (interquartile range). $\mathbf{N} . \mathbf{A} .=$ not applicable; $\mathbf{G y}=$ gray; $\mathbf{T C D}=$ total cumulative dose; $\mathbf{B M I}=$ body mass index. 
At time of the study eight subjects (seven survivors and one control) were treated for hypertension, of whom six survivors and one control were treated with renin-angiotensin-system inhibitors. Five survivors had type 2 diabetes and one survivor had type 1 diabetes, none of the controls had diabetes. Four survivors and none of the controls were treated for dyslipidemia. None of the survivors or controls experienced a cardio- or cerebrovascular incident.

\section{Serum markers}

Triglyceride levels were significantly higher in nephroblastoma survivors (median $1.00 \mathrm{mmol} / \mathrm{l}$ ) than in controls (median $0.79 \mathrm{mmol} / \mathrm{l}$ ) and tended to be higher in neuroblastoma survivors (median $0.90 \mathrm{mmol} / \mathrm{l}$ ) (Table 2). FFA levels were significantly higher in neuroblastoma survivors (median $0.63 \mathrm{mmol} / \mathrm{l}$ ) as compared with controls (median $0.49 \mathrm{mmol} / \mathrm{l}$ ) whereas levels in nephroblastoma survivors (median $0.50 \mathrm{mmol} / \mathrm{l}$ ) were not.

Nephroblastoma survivors had significantly higher cystatin C (median $0.89 \mathrm{mg} / \mathrm{l}$ ), uric acid (median $0.34 \mathrm{mmol} / \mathrm{l}$ ) and urea (median $5.4 \mathrm{mmol} / \mathrm{l}$ ) levels than controls (median 0.80 $\mathrm{mg} / \mathrm{l}, 0.31 \mathrm{mmol} / \mathrm{l}, 5.1 \mathrm{mmol} / \mathrm{l}$, respectively), whereas neuroblastoma survivors did not (Table 2). Protein C and S, vWf, LAdRVV and antithrombin III were all within the normal range in all subjects (data not shown).

\section{Cardiovascular parameters}

After adjusting for age, sex, BMI and smoking, both nephroblastoma and neuroblastoma survivors had higher systolic $(\beta=5.8, P=0.005$ and $\beta=7.3, P=0.003$, respectively) and diastolic ( $\beta=5.0, P=0.001$ and $\beta=5.9, P=0.001$, respectively) blood pressure levels than controls. Abdominal radiotherapy was associated with higher diastolic blood pressure $(\beta=3.9, P=0.041)$.

Nephroblastoma but not neuroblastoma survivos had lower pulse pressure amplification $(\beta=-0.22, P=0.008)$ and higher central systolic blood pressure $(\beta=7.90, P=0.008)$. Abdominal radiotherapy was associated with higher pulse wave velocity $(\beta=0.89, P=0.017)$ and higher central diastolic blood pressure $(\beta=5.96, P=0.006)$ (Table 3).

Two chemotherapeutic agents were associated with several vascular parameters: Cumulative dose of actinomycin was positively associated with pulse pressure $(\beta=0.33, P=0.013)$, pulse wave velocity $(\beta=0.06, P=0.031)$ and central diastolic blood pressure $(\beta=1.00, P=0.001)$ and cumulative dose of cyclophosphamide was positively associated with pulse pressure $(\beta=0.38, P<0.0001)$ and central systolic $(\beta=0.38, P<0.0001)$ and diastolic blood pressure $(\beta=0.35, P=0.007)$. Type of malignancy and treatment components were not associated with intima media thickness or distensibility (Table 3).

LVSF was routinely performed in 26 survivors treated with anthracyclines. Median LVSF in these survivors was $34 \%$ (range $16-65$ ). Two survivors had a LVSF below $20 \%$. Cumulative dose of anthracyclines was significantly correlated with LVSF ( $R=-0.53, P=0.007)$. Subanalyses excluding two subjects with a LVSF below $20 \%$ were performed for all vascular parameters, which did not change the results. Two survivors had a MDRD below $60 \mathrm{~mL} / \mathrm{min} / 1.73 \mathrm{~m}^{2}$, indicating a decreased creatinin clearance. MDRD was not associated with any of the vascular parameters. 
Table 2. Metabolic and vascular parameters survivors and controls.

\begin{tabular}{|c|c|c|c|}
\hline & $\begin{array}{l}\text { Controls } \\
(\mathrm{N}=61)\end{array}$ & $\begin{array}{l}\text { Nephroblastoma } \\
\qquad(\mathrm{N}=67)\end{array}$ & $\begin{array}{l}\text { Neuroblastoma } \\
\qquad(\mathrm{N}=36)\end{array}$ \\
\hline \multicolumn{4}{|l|}{ Anthropometry } \\
\hline BMI $\left(\mathrm{kg} / \mathrm{m}^{2}\right)$ & $24.2(22.1-27.4)$ & $24.6(21.6-27.1)$ & $24.1(20.8-26.0)$ \\
\hline $\mathrm{SBP}(\mathrm{mmHg})$ & $118(111-127)$ & $123(117-133)^{c}$ & $124(114-131)$ \\
\hline $\mathrm{DBP}(\mathrm{mmHg})$ & $72(66-78)$ & $77(73-83)^{c}$ & $75(72-84)^{b}$ \\
\hline \multicolumn{4}{|l|}{ Biomarkers } \\
\hline Triglycerides (mmol/l) & $0.79(0.62-1.20)$ & $1.00(0.79-1.55)^{c}$ & $0.90(0.65-1.26)$ \\
\hline HDL-C (mmol/l) & $1.33(1.13-1.54)$ & $1.36(1.09-1.57)$ & $1.32(1.11-1.52)$ \\
\hline FFA (mmol/l) & $0.49(0.35-0.64)$ & $0.50(0.39-0.63)$ & $0.63(0.45-0.76)^{\mathrm{a}}$ \\
\hline Apo-A1(g/I) & $1.36(1.25-1.50)$ & $1.39(1.24-1.54)$ & $1.33(1.21-1.54)$ \\
\hline Apo-B (g/I) & $0.80(0.68-0.95)$ & $0.85(0.71-1.06)$ & $0.84(0.70-1.07)$ \\
\hline LDL-C (mmol/l) & $2.83(2.25-3.20)$ & $2.83(2.22-3.55)$ & $2.86(2.30-3.56)$ \\
\hline $\operatorname{Lp}(a)(g / l)$ & $0.11(0.06-0.31)$ & $0.13(0.05-0.37)$ & $0.11(0.05-0.36)$ \\
\hline $\mathrm{hsCRP}(\mathrm{mg} / \mathrm{l})$ & $1.27(0.51-3.81)$ & $1.57(0.68-3.51)$ & $1.42(0.42-3.39)$ \\
\hline Fibrinogen (g/l) & $2.9(2.5-3.3)$ & $3.0(2.4-3.3)$ & $2.8(2.5-3.3)$ \\
\hline PAI-1 (IU/ml) & $3.1(0.5-10.4)$ & $6.1(2.3-13.8)$ & $5.8(1.5-15.5)$ \\
\hline Glucose $^{\mathrm{a}}(\mathrm{mmol} / \mathrm{l})$ & $4.9(4.7-5.2)$ & $5.1(4.7-5.5)$ & $4.8(4.5-5.4)$ \\
\hline Insulinª(pmol/l) & $25.0(13.0-34.5)$ & $32.0(13.0-61.0)$ & $13.0(13.0-46)$ \\
\hline HOMA $^{a}$ & $0.50(0.40-0.65)$ & $0.60(0.40-1.10)$ & $0.40(0.40-0.73)$ \\
\hline Leptin (ng/ml) & $7.7(2.4-14.5)$ & $8.9(3.7-16.9)$ & $8.9(5.2-15.2)$ \\
\hline Adiponectin ( $\mu \mathrm{g} / \mathrm{ml})$ & $2.7(1.8-4.3)$ & $2.5(0.9-4.3)$ & $3.0(1.8-4.5)$ \\
\hline Homocystein $(\mu \mathrm{mol} / \mathrm{l})$ & $9.9(8.8-11.6)$ & $10.6(9.3-12.5)$ & $11.3(8.3-14.0)$ \\
\hline Cystatin C (mg/l) & $0.80(0.74-0.86)$ & $0.89(0.83-0.99)^{c}$ & $0.83(0.74-0.87)$ \\
\hline Uric acid (mmol/l) & $0.31(0.26-0.34)$ & $0.34(0.26-0.42)^{b}$ & $0.29(0.22-0.36)$ \\
\hline Urea (mmol/l) & $5.1(4.3-5.8)$ & $5.4(4.7-6.6)^{\mathrm{b}}$ & $5.0(4.5-6.3)$ \\
\hline \multicolumn{4}{|l|}{ Vascular parameters } \\
\hline $\mathrm{PP}(\mathrm{mmHg})$ & $46(42-50)$ & $46(40-53)$ & $45(41-49)$ \\
\hline Central SBP (mmHg) & $110(101-123)$ & $119(108-128)^{b}$ & $109(101-125)$ \\
\hline Central DBP (mmHg) & $77(70-86)$ & $77(72-86)$ & $76(70-84)$ \\
\hline Central PP (mmHg) & $34(28-44)$ & $40(32-49)^{b}$ & $35(30-43)$ \\
\hline PPA & $1.31(1.07-1.69)$ & $1.23(0.98-1.43)$ & $1.33(1.14-1.61)$ \\
\hline Diameter CCA (mm) & $6.4(5.8-6.8)$ & $6.2(5.9-6.8)$ & $6.5(6.1-6.9)$ \\
\hline IMT & $531(474-586)$ & $530(478-604)$ & $523(471-570)$ \\
\hline DC & $28.2(19.0-37.6)$ & $22.0(14.9-32.3)$ & $29.6(20.7-31.9)$ \\
\hline $\mathrm{PWV}(\mathrm{m} / \mathrm{s})$ & $7.0(6.3-7.8)$ & $7.0(6.0-8.2)$ & $6.8(6.0-7.5)$ \\
\hline
\end{tabular}

Data are presented as median and iqr = interquartile range; $\mathbf{B M I}=$ Body mass index; SBP = systolic blood pressure; $\mathbf{D B P}=$ diastolic blood pressure; $\mathbf{P P}=$ Pulse pressure; $\mathbf{P P A}=$ Pulse pressure amplification; $\mathbf{I M T}=$ intima media thickness;

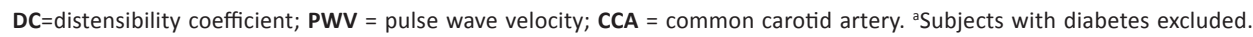
${ }^{b} \mathrm{P}<0.05^{\mathrm{c}} \mathrm{P}<0.01$ (Mann Whitney U-test). 


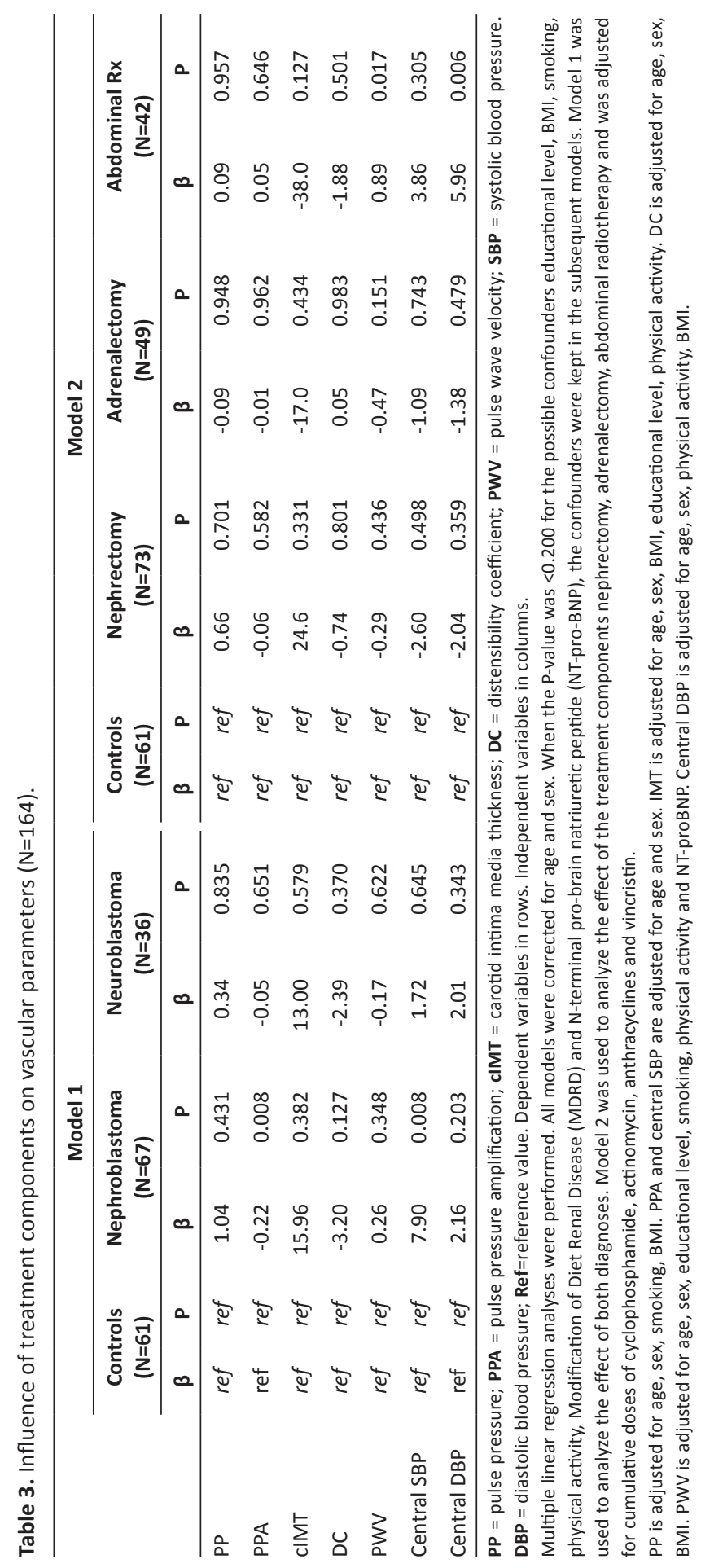




\section{Principal component analysis}

Using PCA, we identified seven underlying components of biomarkers. This seven-component solution explained $68.9 \%$ of the total variance (Table 4). The seven components were given the following names: 1) "Adverse lipids", with positive loadings of LDL-C, apo-B, triglycerides and Ip(a), 2) "Markers for renal function", with positive loadings of cystatin C, uric acid, urea and homocystein, 3) "Insulin resistance", with positive loadings of insulin and Homeostatic Model Assessment (HOMA), 4) "Favourable lipids" with positive loadings of apo-A1 and HDL-C, 5) "Inflammation and coagulation" with positive loadings of hs-CRP and fibrinogen, 6) "Adipocyte derived factors" with positive loadings of PAI-1 and FFA and 7) "Glucose regulation" with a positive loading of glucose and an inverse loading of adiponectin (Table 4).

Table 4. Factor loadings of components.

\begin{tabular}{|c|c|c|c|c|c|c|c|}
\hline & \multicolumn{7}{|c|}{ Principal components } \\
\hline & 1 & 2 & 3 & 4 & 5 & 6 & 7 \\
\hline LDL-C & 907 & 159 & , 049 &,- 056 & ,007 & 054 &,- 016 \\
\hline Apo-B & ,893 & 203 & 108 &,- 018 & ,099 & 123 & ,031 \\
\hline Triglycerides & ,491 & ,260 & 203 &,- 058 & 230 & 308 & 350 \\
\hline $\operatorname{Lp}(a)$ & ,441 &,- 168 &,- 259 &,- 160 &,- 158 &,- 380 & 310 \\
\hline CystatinC & 186 & ,783 &,- 046 & ,097 & 108 &,- 040 &,- 007 \\
\hline Uric acid & ,308 & ,660 & 125 &,- 205 &,- 095 & 193 & 196 \\
\hline Ureum & 236 & 619 & 157 & 105 &,- 084 & 142 &,- 113 \\
\hline Homocystein &,- 156 & ,571 &,- 238 &,- 228 &,- 008 &,- 112 & , 165 \\
\hline Leptin & 247 &,- 379 & 359 & 207 & 266 & 214 & ,066 \\
\hline Insulin & ,057 &,- 005 & 920 &,- 115 & 014 &,- 045 & 082 \\
\hline HOMA & ,067 & ,016 & ,911 &,- 095 & ,078 & ,070 & 121 \\
\hline Apo-A1 & ,030 & ,049 &,- 050 & 909 & 051 & ,031 & ,018 \\
\hline HDL-C &,- 180 &,- 123 &,- 120 & ,878 &,- 146 &,- 134 &,- 034 \\
\hline hs-CRP &,- 120 & ,084 & ,032 &,- 024 & ,845 & ,024 & 121 \\
\hline Fibrinogen & ,217 &,- 138 & ,072 &,- 031 & ,789 & ,009 &,- 025 \\
\hline PAI-1 & 170 & 126 & ,055 &,- 218 &,- 060 & 707 &,- 001 \\
\hline FFA & , 060 &,- 314 &,- 147 & ,339 & 195 & ,534 & 190 \\
\hline Glucose &,- 104 & ,043 & ,236 & 121 &,- 153 & 314 & 690 \\
\hline Adiponectin &,- 145 &,- 040 &,- 039 & ,060 &,- 215 & 128 &,- 654 \\
\hline
\end{tabular}

Component 1: Adverse lipids, Component 2: Markers for impaired renal function, Component 3: Insulin resistance, Component 4: Favourable lipids, Component 5: Inflammation \& coagulation, Component 6: Adipocyte derived factors, Component 7: Glucose regulation 


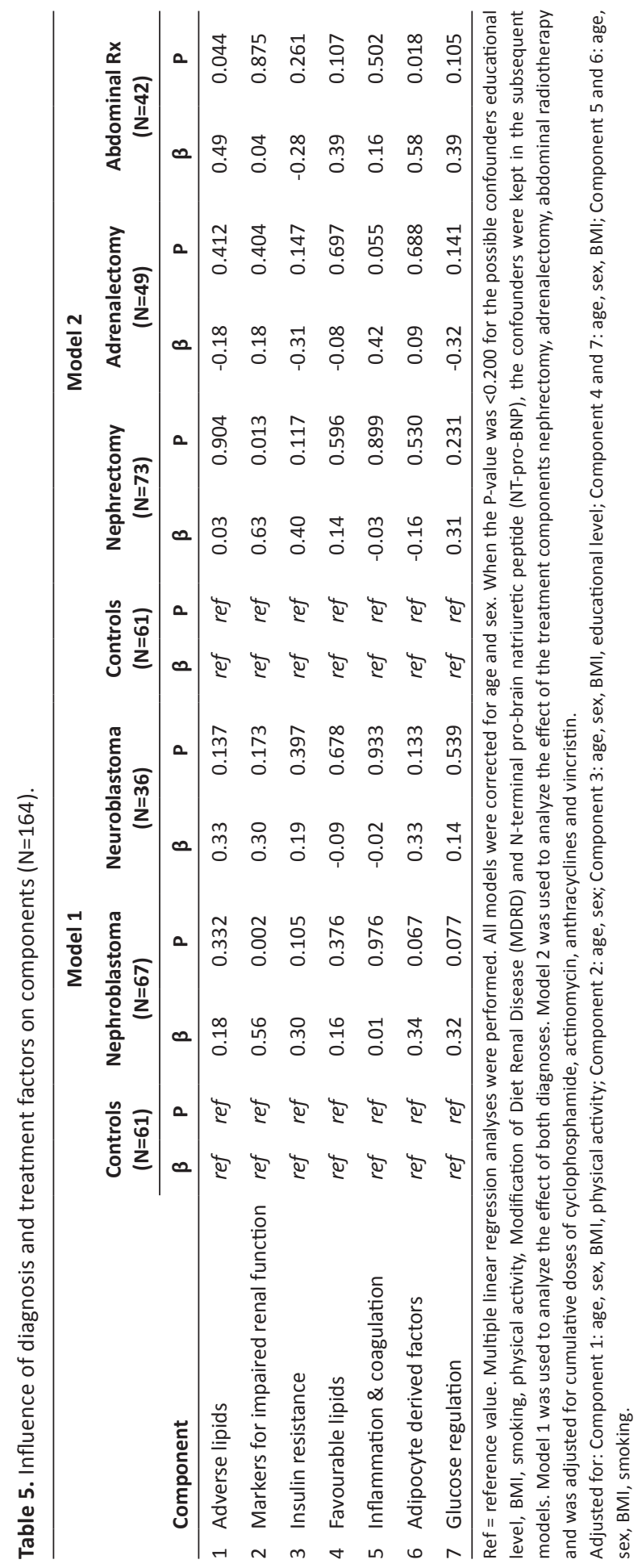


The association of type of malignancy and treatment components with the seven identified components was assessed using multiple linear regression analysis. Abdominal radiotherapy was significantly associated with Component 1 /Adverse lipids $(\beta=0.49, P=0.044)$ and with Component 6/Adipocyte derived components $(\beta=0.58, P=0.018$ ) (Table 5). Nephroblastoma diagnosis $(\beta=0.56, P=0.002$ ) was positively associated with Component $2 /$ Markers for impaired renal function, which was most likely determined by the effect of nephrectomy $(\beta=0.63, P=0.013)$ (Table 5). Diagnoses and treatment components were not associated with Component $3 /$ Insulin resistance, Component 4/Favourable lipids, Component 5/Inflammation and coagulation or Component 7/Glucose regulation (Table 5).

\section{Discussion}

This is the first report that describes an integrated risk profile, using principal component analysis, for cardiovascular diseases in very long-term nephroblastoma and neuroblastoma survivors. We observed nephroblastoma and neuroblastoma survivors, especially after abdominal irradiation, to have increased blood pressure levels and arterial stiffness and concomittant biomarker profiles such as adverse lipids and adipocyte derived factors.

Previous studies have reported an increased mortality risk for nephroblastoma and neuroblastoma survivors $^{36}$ and especially in abdominally irradiated survivors, the Childhood Cancer Survivor Study (CCSS) observed that individuals more often used medication for hypertension and diabetes. Moreover, survivors who received radiation involving both chest and abdomen more often used treatment for dyslipidemia. ${ }^{37}$ Other studies observed that abdominally irradiated nephroblastoma survivors were at higher cardiovascular risk and at increased risk for developing arterial hypertension and renal insufficiency, although dyslipidemia was not reported. ${ }^{3,4}$ This may also be due to the fact that the CCSS results were mainly based on questionnaires and not on laboratory parameters, and that the only other study reporting lipid spectrum did not assess the full lipid spectrum as has been done in the current study.

The other component that was expressed by abdominally irradiated survivors was characterized by adipocyte derived factors, i.e. PAI-1 and FFA. We are the first to report on these components in long-term nephroblastoma and neuroblastoma survivors, although PAI-1 is often mentioned as a component of the metabolic syndrome ${ }^{38}$ and FFA are known to be an important link between obesity and insulin resistance. ${ }^{39}$

Furthermore, diastolic blood pressure and pulse wave velocity were significantly higher in irradiated survivors, indicating that arterial stiffness is increased. Aortic pulse wave velocity is an independent predictor of coronary heart disease and stroke in apparently healthy subjects. ${ }^{40}$ The fact that abdominally irradiated survivors have an increased pulse wave velocity and higher central blood pressure, and express risk profiles characterized by adverse lipids and PAI-1 and FFA, puts them at higher risk for cardiovascular diseases. 
Nephrectomy was significantly associated with a risk profile characterized by high cystatin $C$, uric acid, urea and homocystein, which are associated with impaired renal function and cardiovascular diseases. ${ }^{12-14}$ Adrenalectomy was not directly associated with vascular parameters or identified components of biomarkers. However, as we have previously shown, increased basal cortisol levels as a consequence of adrenalectomy might lead to increased insulin resistance and dyslipidemia. ${ }^{41}$ Furthermore, cumulative doses of actinomycin and cyclophosphamide were associated with features of arterial stiffness. Although the pathogenesis needs to be elucidated, it might very well be that these chemotherapeutic agents damage the vascular endothelium, resulting in vascular alterations.

There are a few limitations of the current study that need to be considered. First, multiple statistical analyses were performed. Performing principal component analysis however enabled us to take into account the effect of a broad spectrum of biomarkers, while reducing multiple testing. Furthermore, the significant results of our study confirm our hypotheses, which indicates that the reported results are not spurious. Finally, this study provides information at one timepoint and in the future measurements of these survivors in time, including also cardiovascular endpoints, will be of importance for understanding the risk factors for childhood cancer survivors.

In conclusion, adult long-term survivors of nephroblastoma and neuroblastoma express several cardiovascular risk factors. Abdominal irradiation and nephrectomy seem to be the most important denominators of risk for cardiovascular diseases. As relatively young aged survivors already express metabolic and vascular alterations, the relevance of these findings for the future is high. Consequently, future prospective studies should therefore follow large cohorts of nephroblastoma and neuroblastoma survivors while ageing. 


\section{Supplementary text}

\section{Laboratory measurements}

Fasting blood samples were taken from an intravenous-cannula before 10:00 a.m. Serum values of triglyceride ( $\mathrm{mmol} / \mathrm{l}), \mathrm{HDL}-\mathrm{C}(\mathrm{mmol} / \mathrm{l}), \mathrm{LDL}-\mathrm{C}(\mathrm{mmol} / \mathrm{l})$, glucose $(\mathrm{mmol} / \mathrm{l})$, free fatty acids (FFA) $(\mathrm{mmol} / \mathrm{l})$, uric acid $(\mathrm{mmol} / \mathrm{l})$ and urea $(\mathrm{mmol} / \mathrm{l})$ were measured using an enzymatic in vitro assay (Roche Diagnostics, Mannheim, Germany). Serum insulin levels (pmol/l) were measured using a chemi-luminescence-based immunoassay (Immulite 2000, Siemens DPC, Los Angeles, CA, USA). Homeostatic model assessment (HOMA), that quantifies insulin resistance and beta cell function, was calculated. ${ }^{24}$ Apolipoprotein-A1 (Apo-A1) (g/l) and Apo-B (g/l) were measured by kinetic immunonephelometry (IMMAGE ${ }^{\circledR}$ Immunochemistry System, Beckman Coulter, CA, USA). Leptin $(\mathrm{ng} / \mathrm{ml})$ was measured using a radioimmunoassay kit (Millipore, MO, USA) and high molecular weight $(\mathrm{HMW})$ adiponectin $(\mu \mathrm{g} / \mathrm{ml})$ using a mulitimeric enzyme-linked immunosorbent assay (ALPCO Diagnostics, MA, USA). Homocystein ( $\mu \mathrm{mol} / \mathrm{I}$ ) was measured using high-performance liquid chromatography method with fluorescence detection. Antithrombin III (IU/ml) (Chromogenix, ILC, Lexington, MA, USA), Protein C activity (IU/ml) (Dade Behring-Siemens), Protein S activity (IU/ $\mathrm{ml}$ ) (Roche Diagnostics), Lupus Anticoagulans diluted Russell's viper venom time (sec) (LAdRVVT; Dade Behring-Siemens) and fibrinogen ( $\mathrm{g} / \mathrm{l}$ ) according to Clauss were performed on Sysmex CA1500 (Siemens HealthCare Diagnostics, Breda, the Nederlands). Von Willebrandfactor antigen (vWf-ag) (E/ml) was determined with an in-house ELISA with polyclonal rabbit anti-human vWf anti-bodies and horseradish peroxidase conjugated anti-human vWf (DakoCytomation, Glostrop, Denmark) for catching and tagging, respectively. Protein C and S, vWf, LAdRVV and antithrombin were measured to exclude subjects with possible coagulation problems and were not reported as outcome variables. PAI-1 (IU/ml) was determined by a two-stage, indirect enzymatic assay (Biopool, Trinity Biotech, Dublin, Ireland). Cystatin C (mg/l) was measured on a Roche Modular P800 (Roche, Almere, The Netherlands). Serum levels of lipoprotein a (Ip(a)) (mmol/l) and highsensitivity $\mathrm{C}$ reactive protein ( $\mathrm{hsCRP}$ ) ( $\mathrm{mg} / \mathrm{l})$ were routinely measured on a Beckman Immage nephelometer (Beckman Coulter, Woerden, The Netherlands). Modification of Diet in Renal Disease (MDRD) study equation, the gold standard for creatinin clearance was calculated and MRDR below $60 \mathrm{~mL} / \mathrm{min} / 1.73 \mathrm{~m}^{2}$ was considered abnormal. ${ }^{25,26}$ Serum $\mathrm{N}$-terminal pro-brain natriuretic peptide (NT-pro-BNP) (pmol/l) was determined as a marker for heart function and was measured using electrochemiluminescence immunoassay on a Roche Modular E170 (Roche, Almere, The Netherlands). ${ }^{27}$ 


\section{References}

1. Bernstein ML, Leclerc JM, Bunin G, et al. A population-based study of neuroblastoma incidence, survival, and mortality in North America. J Clin Oncol 1992;10:323-9.

2. Bernstein L, Linet $M$, Smith $M$, Olshan AF. Cancer incidence and survival among children and adolescents: United States SEER Program 1975-1995, SEER Program. National Cancer Institute 1999 1999:p.79-90.

3. Geenen MM, Bakker PJ, Kremer LC, Kastelein JJ, van Leeuwen FE. Increased prevalence of risk factors for cardiovascular disease in long-term survivors of acute lymphoblastic leukemia and Wilms tumor treated with radiotherapy. Pediatr Blood Cancer 2010;55:690-7.

4. van Dijk IW, Oldenburger F, Cardous-Ubbink MC, et al. Evaluation of late adverse events in long-term wilms' tumor survivors. Int J Radiat Oncol Biol Phys 2010;78:370-8.

5. Mulrooney DA, Yeazel MW, Kawashima T, et al. Cardiac outcomes in a cohort of adult survivors of childhood and adolescent cancer: retrospective analysis of the Childhood Cancer Survivor Study cohort. BMJ 2009;339:b4606.

6. Pizzo PA, Poplack DG. Principles and Practice of Pediatric Oncology. Philadelphia, PA: Lippincott Williams\&Wilkins;. 2002.

7. Polak JF, Pencina MJ, Pencina KM, O’Donnell CJ, Wolf PA, D'Agostino RB, Sr. Carotid-wall intima-media thickness and cardiovascular events. N Engl J Med 2011;365:213-21.

8. Safar ME, Jankowski P. Central blood pressure and hypertension: role in cardiovascular risk assessment. Clin Sci (Lond) 2009;116:273-82.

9. Roman MJ, Devereux RB, Kizer JR, et al. Central pressure more strongly relates to vascular disease and outcome than does brachial pressure: the Strong Heart Study. Hypertension 2007;50:197-203.

10. Franklin SS, Khan SA, Wong ND, Larson MG, Levy D. Is pulse pressure useful in predicting risk for coronary heart Disease? The Framingham heart study. Circulation 1999;100:354-60.

11. Nijdam ME, Plantinga $\mathrm{Y}$, Hulsen HT, et al. Pulse pressure amplification and risk of cardiovascular disease. Am J Hypertens 2008;21:388-92.

12. Lippi G, Montagnana M, Franchini M, Favaloro EJ, Targher G. The paradoxical relationship between serum uric acid and cardiovascular disease. Clin Chim Acta 2008;392:1-7.

13. Guven A, Inanc F, Kilinc M, Ekerbicer H. Plasma homocysteine and lipoprotein (a) levels in Turkish patients with metabolic syndrome. Heart Vessels 2005;20:290-5.

14. Shlipak MG, Sarnak MJ, Katz R, et al. Cystatin C and the risk of death and cardiovascular events among elderly persons. N Engl J Med 2005;352:2049-60.

15. Martin SS, Qasim A, Reilly MP. Leptin resistance: a possible interface of inflammation and metabolism in obesity-related cardiovascular disease. J Am Coll Cardiol 2008;52:1201-10.

16. Im JA, Kim SH, Lee JW, Shim JY, Lee HR, Lee DC. Association between hypoadiponectinemia and cardiovascular risk factors in nonobese healthy adults. Metabolism 2006;55:1546-50.

17. Herder C, Karakas M, Koenig W. Biomarkers for the prediction of type 2 diabetes and cardiovascular disease. Clin Pharmacol Ther 2011;90:52-66.

18. Sakkinen PA, Wahl P, Cushman M, Lewis MR, Tracy RP. Clustering of procoagulation, inflammation, and fibrinolysis variables with metabolic factors in insulin resistance syndrome. Am J Epidemiol 2000;152:897-907.

19. Meigs JB, D'Agostino RB, Sr., Wilson PW, Cupples LA, Nathan DM, Singer DE. Risk variable clustering in the insulin resistance syndrome. The Framingham Offspring Study. Diabetes 1997;46:1594-600.

20. Kekalainen P, Sarlund H, Pyorala K, Laakso M. Hyperinsulinemia cluster predicts the development of type 2 diabetes independently of family history of diabetes. Diabetes Care 1999;22:86-92.

21. Gray RS, Fabsitz RR, Cowan LD, Lee ET, Howard BV, Savage PJ. Risk factor clustering in the insulin resistance syndrome. The Strong Heart Study. Am J Epidemiol 1998;148:869-78.

22. Assembly WMAG. Declaration of Helsinki - Sixth revision. 2008. 
23. Wendel-Vos GC, Schuit AJ, Saris WH, Kromhout D. Reproducibility and relative validity of the short questionnaire to assess health-enhancing physical activity. J Clin Epidemiol 2003;56:1163-9.

24. Matthews DR, Hosker JP, Rudenski AS, Naylor BA, Treacher DF, Turner RC. Homeostasis model assessment: insulin resistance and beta-cell function from fasting plasma glucose and insulin concentrations in man. Diabetologia 1985;28:412-9.

25. Foundation NK. K/DOQI clinical practice guidelines for chronic kidney disease: Part 5: evaluation of laboratory measurements for clinical assessment of kidney disease. Guideline 4: estimation of GFR. . American Journal Kidney Diseases 2002;39.

26. Levey AS, Eckardt KU, Tsukamoto Y, et al. Definition and classification of chronic kidney disease: a position statement from Kidney Disease: Improving Global Outcomes (KDIGO). Kidney Int 2005;67:2089-100.

27. Januzzi JL, van Kimmenade R, Lainchbury J, et al. NT-proBNP testing for diagnosis and short-term prognosis in acute destabilized heart failure: an international pooled analysis of 1256 patients: the International Collaborative of NT-proBNP Study. Eur Heart J 2006;27:330-7.

28. Laurent S, Cockcroft J, Van Bortel L, et al. Expert consensus document on arterial stiffness: methodological issues and clinical applications. Eur Heart J 2006;27:2588-605.

29. Reneman RS, van Merode T, Hick P, Muytjens AM, Hoeks AP. Age-related changes in carotid artery wall properties in men. Ultrasound Med Biol 1986;12:465-71.

30. Asmar R, Benetos A, Topouchian J, et al. Assessment of arterial distensibility by automatic pulse wave velocity measurement. Validation and clinical application studies. Hypertension 1995;26:485-90.

31. Mattace-Raso FU, van der Cammen TJ, van der Meer IM, et al. C-reactive protein and arterial stiffness in older adults: the Rotterdam Study. Atherosclerosis 2004;176:111-6.

32. Karamanoglu M, O'Rourke MF, Avolio AP, Kelly RP. An analysis of the relationship between central aortic and peripheral upper limb pressure waves in man. Eur Heart J 1993;14:160-7.

33. Carvalho JS, Silva CM, Shinebourne EA, Redington AN. Prognostic value of posterior wall thickness in childhood dilated cardiomyopathy and myocarditis. Eur Heart J 1996;17:1233-8.

34. Harrell jr. FE. Regression Modelling Strategies. Chapter 3. Missing Data. Springer 2001:Page 41-52.

35. DiStefano C, Zhu M, D. M. Understanding and Using Factor Scores: Considerations for the Applied Researcher. Practical Assessment, Research \& Evaluation 2009;14.

36. Mertens AC, Liu Q, Neglia JP, et al. Cause-specific late mortality among 5-year survivors of childhood cancer: the Childhood Cancer Survivor Study. J Natl Cancer Inst 2008;100:1368-79.

37. Meacham LR, Chow EJ, Ness KK, et al. Cardiovascular risk factors in adult survivors of pediatric cancer-a report from the childhood cancer survivor study. Cancer Epidemiol Biomarkers Prev 2010;19:17081.

38. Alessi MC, Juhan-Vague I. PAI-1 and the metabolic syndrome: links, causes, and consequences. Arterioscler Thromb Vasc Biol 2006;26:2200-7.

39. Boden G. Free fatty acids, insulin resistance, and type 2 diabetes mellitus. Proc Assoc Am Physicians 1999;111:241-8.

40. Mattace-Raso FU, van der Cammen TJ, Hofman A, et al. Arterial stiffness and risk of coronary heart disease and stroke: the Rotterdam Study. Circulation 2006;113:657-63.

41. Van Waas M, Pieters R, van Eck JP, et al. Adrenal function in adult long-term survivors of nephroblastoma and neuroblastoma. Eur J Cancer 2012;48:1159-66. 



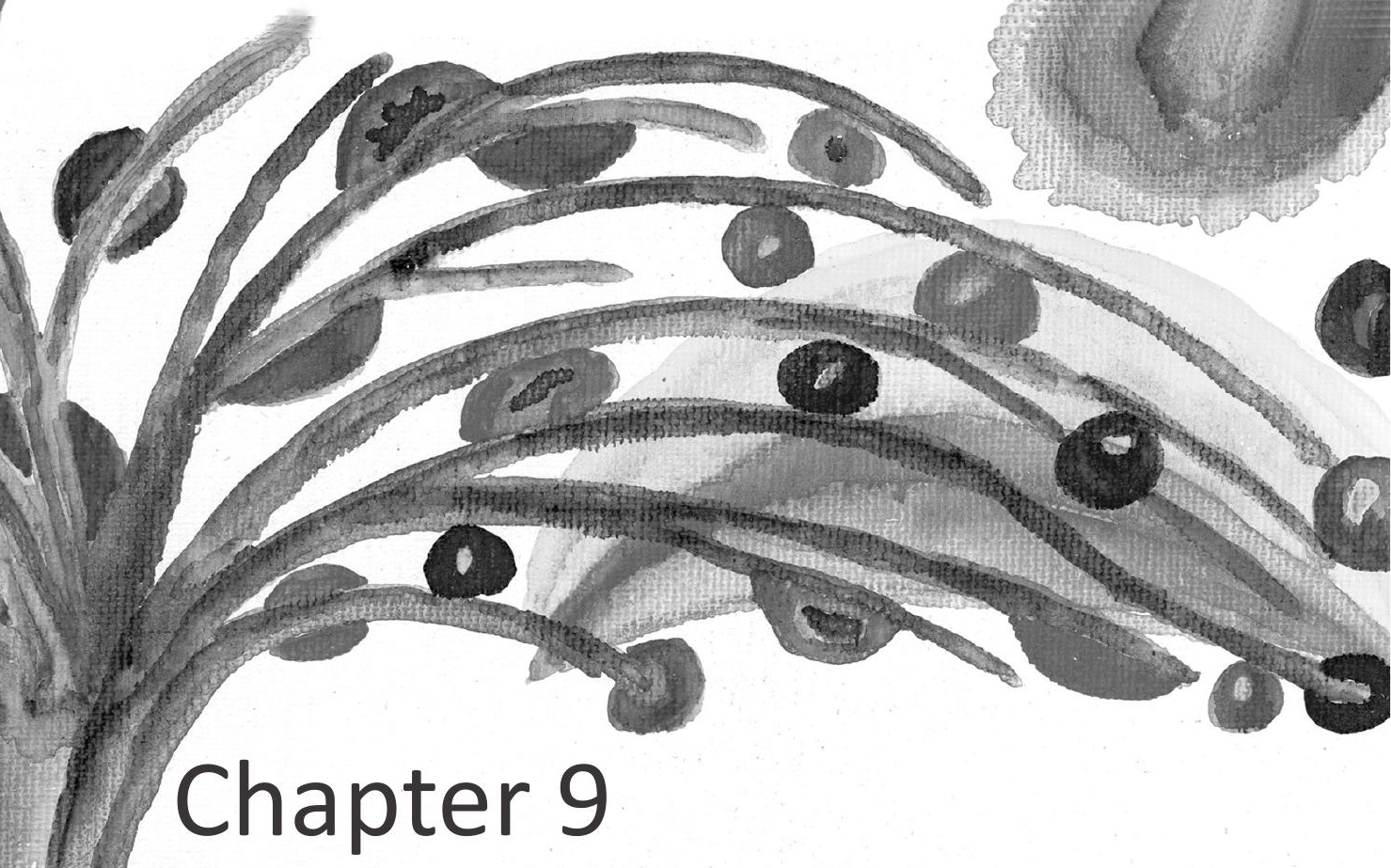

Treatment factors rather than genetic variation determine metabolic syndrome in childhood cancer survivors

Marjolein van Waas ${ }^{1}$, Sebastian J.C.M.M. Neggers ${ }^{1,2}$, André G. Uitterlinden ${ }^{3}$, Karin Blijdorp ${ }^{1,2}$, Rob Pieters ${ }^{1}$, Marry M. van den Heuvel-Eibrink ${ }^{1}$

${ }^{1}$ Department of Pediatric Oncology/Hematology, Erasmus MC-Sophia Children's Hospital, Rotterdam, the Netherlands

${ }^{2}$ Department of Medicine, Section Endocrinology, Erasmus University Medical Centre, Rotterdam, the Netherlands

${ }^{3}$ Department of Internal Medicine, Erasmus University Medical Centre, Rotterdam, the Netherlands 


\section{Abstract}

Background: Genetic variation that regulates insulin resistance, blood pressure and adiposity in the normal population might determine differential vulnerability for metabolic syndrome after treatment for childhood cancer. Aim was to evaluate the contribution of candidate single nucleotide polymorphisms (SNPs) relevant for metabolic syndrome in our single centre cohort of adult long-term childhood cancer survivors.

Patients and methods: In this retrospective study 532 survivors were analyzed. Median age at diagnosis was 5.7 (range $0.0-17.8$ ) years, median follow-up time was 18.2 (range 5.0-48.8) years and median age at follow-up was 26.2 (range 18.0 - 50.8). JAZF1 gene rs864745, THADA gene rs7578597, IRS1 gene rs2943641, TFAP2B gene rs987237, MSRA gene rs7826222, ATP2B1 gene rs2681472 and rs2681492 were genotyped. The association of genotypes with total cholesterol levels, blood pressure, body mass index, waist circumference and frequency of diabetes were assessed.

Results: Metabolic syndrome was more frequent in cranially $(23.3 \%, \mathrm{P}=0.002)$ and abdominally (23.4\%, $\mathrm{P}=0.009$ ) irradiated survivors as compared with non-irradiated survivors $(10.0 \%)$. Association of allelic variants in rs2681472 and rs2681492 with hypertension, rs987237 and rs7826222 with waist circumference and rs864745, rs7578597 and rs2943641 with diabetes were not significant. None of the SNPs was associated with the metabolic syndrome. Adjusting for age, sex, follow-up time, cranial irradiation and abdominal irradiation did not change these results.

Conclusions: Treatment factors rather than genetic variation determine hypertension, waist circumference, diabetes and metabolic syndrome in adult long-term survivors of childhood cancer. 


\section{Introduction}

Hypertension, adiposity, dyslipidemia and insulin resistance, when occurring together referred to as the metabolic syndrome, have been reported frequently in childhood cancer survivors. ${ }^{1-6}$ Metabolic syndrome is known to increase the risk for cardiovascular diseases and type 2 diabetes mellitus. ${ }^{7}$ In particular survivors treated with cranial irradiation, total body irradiation or abdominal irradiation seem to be at risk for developing (components of) the metabolic syndrome. ${ }^{4-6}$

Variation of long-term toxicity in equally treated childhood cancer survivors suggests that genetic variation and environmental factors may influence the occurrence and the level/impact of late effects. Single nucleotide polymorphisms (SNPs) are DNA sequence variations that occur when a single nucleotide in the genome sequence is altered. SNPs determine about $90 \%$ of all human genetic variation. Large-scale genome-wide association studies (GWAS), in which hundreds of thousands common genetic variants are genotyped, identified genes associated with common diseases and traits. ${ }^{8,9}$ The ATP2B1 gene appeared to be associated with systolic and diastolic blood pressure and hypertension. ${ }^{10}$ This gene encodes PMCA1, a plasma membrane calcium/calmodulin dependent ATP-ase that is expressed in vascular endothelium and is involved in calcium pumping from the cytosol to the extracellular component. ${ }^{11}$ Two loci near TFAP2B and MRSA influence adiposity and fat distribution through an effect on overall obesity or fat mass. ${ }^{12}$ A SNP located adjacent to the insulin receptor substrate 1 gene (IRS1) is associated with insulin resistance, hyperinsulinemia and reduced IRS1 protein levels and decreased insulin induction of IRS1-associated phosphatidylinositol-3-OH kinase activity in human skeletal muscle. ${ }^{13}$ Furthermore, loci at the JAZF1 and THADA gene regions have been associated with diabetes..$^{14,15}$

The aim of the current study was to evaluate whether SNPs that have been associated with blood pressure, adiposity and diabetes influence the occurrence of the metabolic syndrome in adult long-term childhood cancer survivors.

\section{Patients and methods}

\section{Patients}

This retrospective study was performed in a single centre cohort of adult long-term childhood cancer survivors treated at the ErasmusMC - Sophia Children's Hospital from October 1960 to June 2004. Survivors were younger than 18 years at the time of diagnosis and were at least 5 years after cessation of treatment when they visited the outpatient clinic for long-term side effects of cancer treatment.

To minimize ethnic influences on the study outcome, only Caucasian subjects were included in this study. Full details on cancer treatment were previously recorded: type and cumulative dose of chemotherapy, site, field and cumulative dose of radiotherapy, extent of surgery, conditioning regimen prior to stem cell transplantation (SCT), complications and relapses. During their regular 
visit at the outpatient clinic general health screening, including extensive history and physical examination, was performed. Written informed consent was obtained from all participants.

\section{Components of the metabolic syndrome}

Height was measured to the nearest millimeter using a Harpenden Stadiometer and weight was assessed but in underwear only to the nearest $0.1 \mathrm{~kg}$ with a standard clinical balance. Body mass index (BMI) was calculated as weight in kilograms divided by the squared length in meters. Waist circumference was measured to the nearest $1 \mathrm{~cm}$, midway between last rib and the iliac crest, hip circumference was measured at the maximum circumference of the buttocks to the nearest $1 \mathrm{~cm}$ and waist-hip-ratio was calculated. Blood pressure was measured on the right arm of the patient with the Dinamap ${ }^{\circledR}$ Procare. Medication use was routinely asked for during visits and was abstracted from the medical records. Medication was categorized in treatment for diabetes, antihypertensive treatment and lipid-lowering treatment. Non-fasting blood samples were taken to assess total cholesterol levels using an enzymatic in vitro assay (Roche Diagnostics, Mannheim, Germany).

Due to the unavailability of fasting lipid spectrum and fasting glucose in our patients, the modified definition of the metabolic syndrome as proposed by Haugnes et al. ${ }^{16}$ was used, as previously described. ${ }^{1}$ Subjects with at least two of the following components were diagnosed with metabolic syndrome: Blood pressure $\geq 140 / 90 \mathrm{mmHg} ; B M I \geq 30 \mathrm{~kg} / \mathrm{m}^{2}$; self reported prevalence of diabetes or medication; serum total cholesterol $\geq 5.2 \mathrm{mmol} / \mathrm{l}$ or medication. Prevalence of the metabolic syndrome was assessed in the total group and in diagnosis subgroups containing at least 30 survivors. Moreover, prevalence of the metabolic syndrome was assessed in radiotherapy subgroups (survivors treated with cranial or abdominal irradiation).

\section{Genotyping}

Seven SNPs were selected based on previous candidate genes studies and GWA studies, ${ }^{10,13,18,19}$ i.e.: JAZF1 gene rs864745, THADA gene rs7578597, IRS1 gene rs2943641 that were previously shown to determine insulin resistance and type 2 diabetes, TFAP2B gene rs987237 and MSRA gene rs7826222 associated with waist circumference and ATP2B1 gene rs2681472 and rs2681492 that are related to systolic and diastolic blood pressure, respectively. We additionally assessed the association of TFAP2B gene rs987237 and MSRA gene rs7826222 with obesity. Finally, the associations of all SNPs with the frequency of the metabolic syndrome were assessed.

Genomic DNA was extracted from peripheral blood using standard DNA extraction methods. 1 - 2 ng genomic DNA was dispensed into 384-wells plates using a Caliper Sciclone ALH3000 pipetting robot (Caliper LS, Mountain View, CA, USA). Genotypes were determined using Sequenom iPLEX genotyping and Taqman Allelic Discrimination assays. All used primers and probers are available on request.

Multiplex PCR assays were designed for the Sequenom iPLEX genotyping using Assay Designer on the website https://mysequenom.com/tools/genotyping/deafult.aspx). For this, sequences 
containing the SNP site and at least 100bp of flanking sequence on either side of the SNP were used. As previously described, ${ }^{20} 2 \mathrm{ng}$ genomic DNA was amplified in a $5 \mu \mathrm{l}$ reaction containing $1 \mathrm{x}$ Taq PCR buffer (Sequenom), $2 \mathrm{mM} \mathrm{MgCl}, 500 \mu \mathrm{M}$ each dNTP, $100 \mathrm{nM}$ each PCR primer, $0.5 \mathrm{U}$ Taq (Sequenom). The reaction was incubated at $94^{\circ} \mathrm{C}$ for 20 seconds, $56^{\circ} \mathrm{C}$ for 30 seconds, $72^{\circ} \mathrm{C}$ for 1 minute, followed by three minutes at $72^{\circ} \mathrm{C}$. Excess dNTPs were then removed from the reaction by incubation with $0.3 \mathrm{U}$ shrimp alkaline phosphatase (Sequenom) at $37^{\circ} \mathrm{C}$ for 40 minutes followed by 5 minutes at $85^{\circ} \mathrm{C}$ to deactivate the enzyme. Single primer extension over the SNP was carried out in a final concentration between $0.731 \mu \mathrm{M}$ and $1.462 \mu \mathrm{M}$ for each extension primer (depending on the mass of the probe), iPLEX termination mix (Sequenom), 10x iPLEX Buffer Plus and iPLEX enzyme (Sequenom) and subsequently cycled using the following program: $94^{\circ} \mathrm{C}$ for 30 seconds followed by $94^{\circ} \mathrm{C}$ for 5 seconds, 5 cycles of $52^{\circ} \mathrm{C}$ for 5 seconds, and $80^{\circ} \mathrm{C}$ for 5 seconds, last three steps were repeated 40 stops, then $72^{\circ} \mathrm{C}$ times for 3 minutes. This reaction was then desalted by addition of $6 \mathrm{mg}$ clear resin (Sequenom) followed by mixing (15 minutes) and centrifugation ( 5 minutes, $3000 \mathrm{rpm}$ ) to settle the contents of the tube. The extension product was then spotted into a 384-wells spectroCHIP using the SEQUENOM MasSARRAY Nanodispenser RS1000 before analysis on the MassARRAY Compact System (Sequenom). Data was collected using SpectroACQUIRE 3.3.1.13. and clustering was called using TYPER analyser 4.03.18 (Sequenom). Additionally, to ensure data quality, genotypes for each subjects were also checked manually.

Genotypes for rs864745 (JAZF1 gene), rs7578597 (THADA gene), rs2943641 (IRS1 gene), rs987237 (TFAP2B gene), rs7826222 (MSRA gene), rs2681472 and rs2681492 (both ATP2B1 gene) were generated using Taqman Allelic Discrimination (Applied Biosystems Inc., Foster City, CA, USA). Primer and probe sequences were optimized using the SNP assay-by-design service of Applied Biosystems. For details see http://www.appliedbiosystems.com. Reactions were performed on the Taqman Prism 7900 HT 384 wells format. All SNPs were tested for HardyWeinberg equilibrium (HWE) within the total group of childhood cancer survivors. As seven SNPS were tested, SNPs with P-values below 0.007 (0.05/7) were considered to be not in HWE.

\section{Statistics}

Statistical analyses were performed with the Statistical Package for Social Sciences (SPSS 17.0, Chicago, IL, USA). The following comparisons were made: Subjects using medication for diabetes versus subjects not using medication for diabetes, subjects with blood pressure levels lower than 140/90 $\mathrm{mmHg}$ versus subjects with blood pressure levels higher than 140/90 $\mathrm{mmHg}$, subjects with total cholesterol levels lower than $5.2 \mathrm{mmol} / \mathrm{l}$ and subjects with total cholesterol levels higher than $5.2 \mathrm{mmol} / \mathrm{l}$, subjects with BMI lower than 30 versus subjects with BMI higher than 30, subjects with low waist circumference (below $88 \mathrm{~cm}$ for females, below $102 \mathrm{~cm}$ for males) versus high waist circumference. Moreover, subjects with the metabolic syndrome were compared with subjects without the metabolic syndrome. Chi-squared tests were used to assess the association between the SNPs and the subgroups based on outcome parameters. Logistic 
regression was performed to assess the influence of rs864745, rs7578597 and rs2943641 on diabetes, of rs987237 and rs7826222 on high waist circumference and of rs2681472 and rs2681492 on hypertension and of all seven SNPs on metabolic syndrome. All logistic regression analyses were adjusted for age, sex, follow-up time, abdominal irradiation and cranial irradiation. Odds ratios (OR) were not calculated when frequencies were below $N=5$. P-values $<0.05$ (twotailed) were considered to be statistically significant.

\section{Results}

\section{Survivors}

Of 712 childhood cancer survivors registered in our outpatient clinic registry, 119 survivors were excluded because none of the components of the metabolic syndrome was available for these subjects. Sixty-one survivors were non-Caucasian and were therefore excluded from this study, leaving 532 survivors eligible for analysis. Median age at diagnosis was 5.7 (range $0.0-$ 17.8) years, median follow-up time was $18.2(5.0$ - 48.8) years and median age at follow-up was $26.2(18.0-50.8)$ (Table 1). Thirteen subgroups according to type of malignancy were present (Table 1).

Both chemotherapy and radiotherapy were administered in 154 (26\%) survivors, 362 (61\%) received chemotherapy only, $26(4 \%)$ received radiotherapy only and $50(8 \%)$ surgery only. Eighty-nine (15\%) survivors received cranial irradiation and $53(9 \%)$ received abdominal irradiation. Twelve (2\%) survivors were treated with allogeneic stem cell transplantation and in six (1\%) treatment included autologous stem cell transplantation. Number of subjects, sex, age, age at diagnosis and follow-up time are summarized per malignancy and therapy subgroup in Table 1. Survivors treated with cranial irradiation (median age 31.0, $\mathrm{P}<0.001$ ) or abdominal irradiation (median age 36.0, $\mathrm{P}<0.001$ ) were significantly older than survivors not treated with radiotherapy (median age 24.4 ) (Table 1 ).

\section{Components of the metabolic syndrome.}

Median and interquartile ranges for systolic and diastolic blood pressure, BMI, waist circumference and total cholesterol levels are shown in Table 1. Five out of 532 (0.9\%) survivors had type 2 diabetes. Twenty percent $(106 / 522)$ of the survivors suffered from arterial hypertension. Nine percent $(47 / 519)$ of the survivors was obese (BMI $\geq 30)$ and $13 \%(57 / 425)$ of the survivors had a high waist circumference. Thirty-six percent (187/523) of the survivors had serum total cholesterol levels higher than $5.2 \mathrm{mmol} / \mathrm{l}$. In 460 of the survivors the metabolic syndrome was assessed: $15 \%$ (67/460) of the survivors had the metabolic syndrome. 


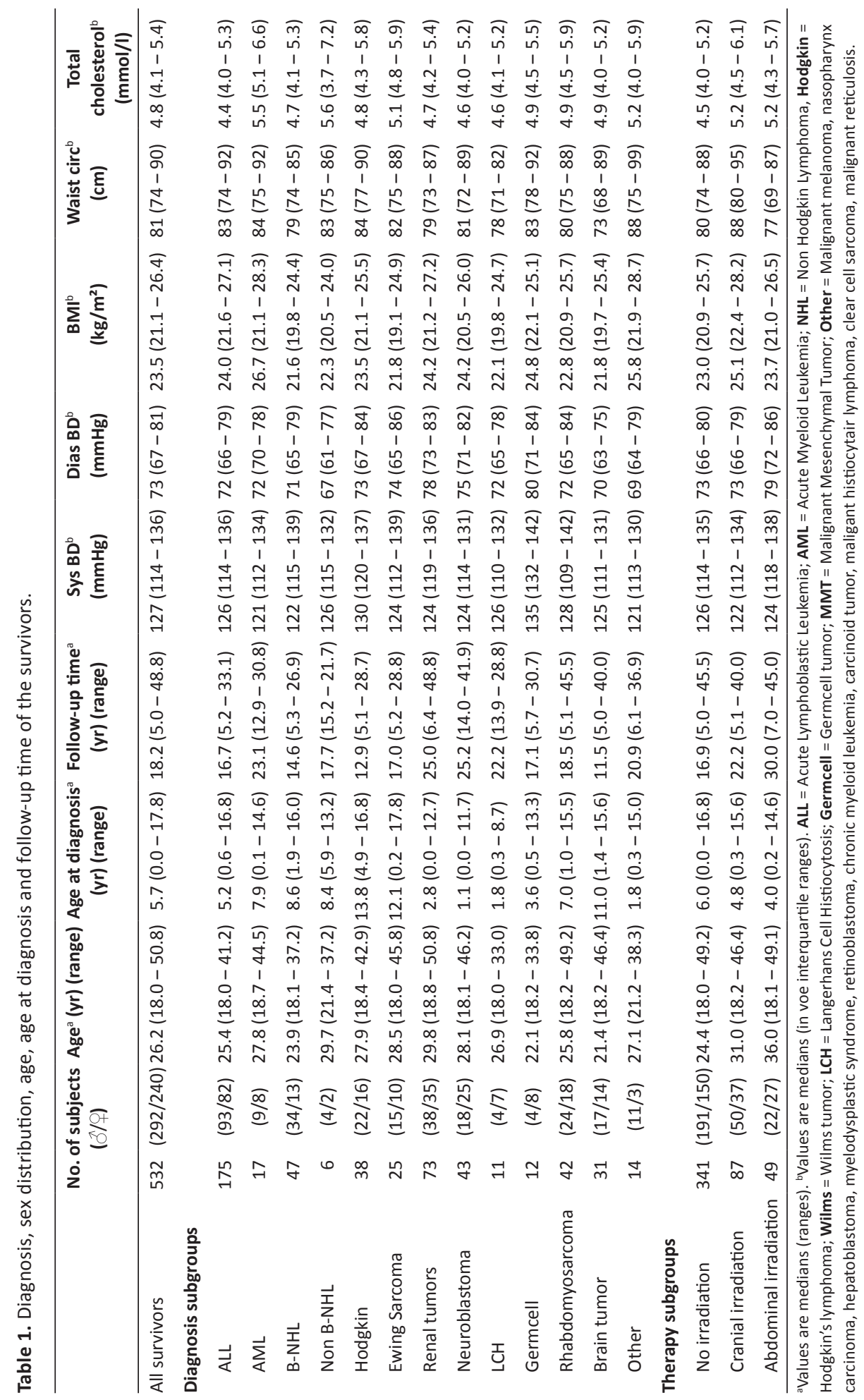


Diabetes was more frequent in Wilms survivors compared with other survivors (3/73 vs. 2/459, $\mathrm{P}=0.002$ ) and more frequent in abdominally irradiated survivors compared with survivors treated without radiotherapy ( $6.1 \%$ vs. $0.3 \%, \mathrm{P}=0.007$ ) (Supplementary tables). High waist circumference was more frequent in brain tumor survivors compared with other survivors $(29.2 \%$ vs. $12.5 \%$, $\mathrm{P}=0.030$ ) and more frequent in survivors treated with cranial irradiation compared with nonirradiated survivors ( $25.0 \%$ vs. $10.5 \%, \mathrm{P}=0.002)$. Brain tumor survivors more frequently had high total cholesterol levels $(58.6 \%$ vs. $34.4 \%, \mathrm{P}=0.015)$. Survivors treated with abdominal irradiation and survivors treated with cranial irradiation more frequently had high total cholesterol levels ( $54.2 \%$ vs. $27.1 \%, \mathrm{P}<0.001$ and $53.5 \%$ vs. $27.2 \%, \mathrm{P}<0.001$, respectively). The metabolic syndrome was more frequent in survivors treated with cranial irradiation $(23.3 \%, \mathrm{P}=0.002)$ and in survivors treated with abdominal irradiation $(23.4 \%, \mathrm{P}=0.009)$ as compared with non-irradiated survivors (10.0\%) (Supplementary tables).

\section{Genotype and fenotype}

Allele frequencies of the seven SNPs are shown in Table 2. All SNPs were in HWE (Table 2). Frequencies of CT in rs2681472 (26\% vs. $29 \%$ ) and GA in rs2681492 (28\% vs. $29 \%$ ) were not different in survivors with hypertension and survivors without hypertension. Frequencies of AG in rs987237 (32\% vs. $29 \%$ ) and CG in rs7826222 (26\% vs. $29 \%$ ) were not different between survivors with high waist circumference versus survivors with normal waist circumference. For the heterozygote alleles and the homozygote minor alleles in the rs864745, rs7578597 and rs2943641 SNPs significance could not be determined as subgroups were smaller than 5 (referred to as "N.A.") (Table 3). Frequencies of CT (52\% vs. 44\%) in rs2681472, GA (15\% vs. $20 \%)$ in rs 2681492 , AG (39\% vs. $42 \%)$ in rs987237, CG (36\% vs. $28 \%)$ in rs7826222, AG $(21 \%$ vs. $31 \%$ ) and GG ( $6 \%$ vs. $2 \%$ ) in rs 864745 , TC ( $28 \%$ vs. $28 \%$ ) in rs 7578597 and CT ( $29 \%$ vs. $28 \%$ ) in rs2943641 were not different between survivors with and without the metabolic syndrome (Table 3). For the homozygote minor alleles the significance of the SNPs for hypertension and waist circumference could not be determined as subgroups were smaller than 5 (referred to as "N.A.") (Table 3).

Adjusting for age, sex, follow-up time, cranial irradiation and abdominal irradiation did not change these results (Table 3 ). 


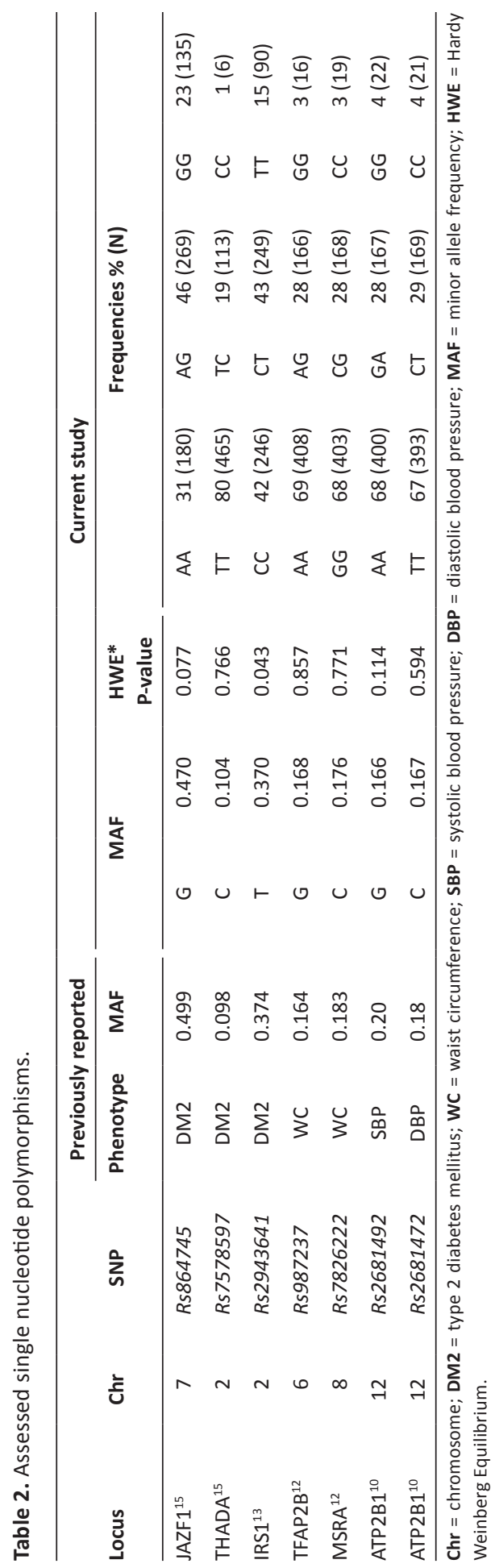




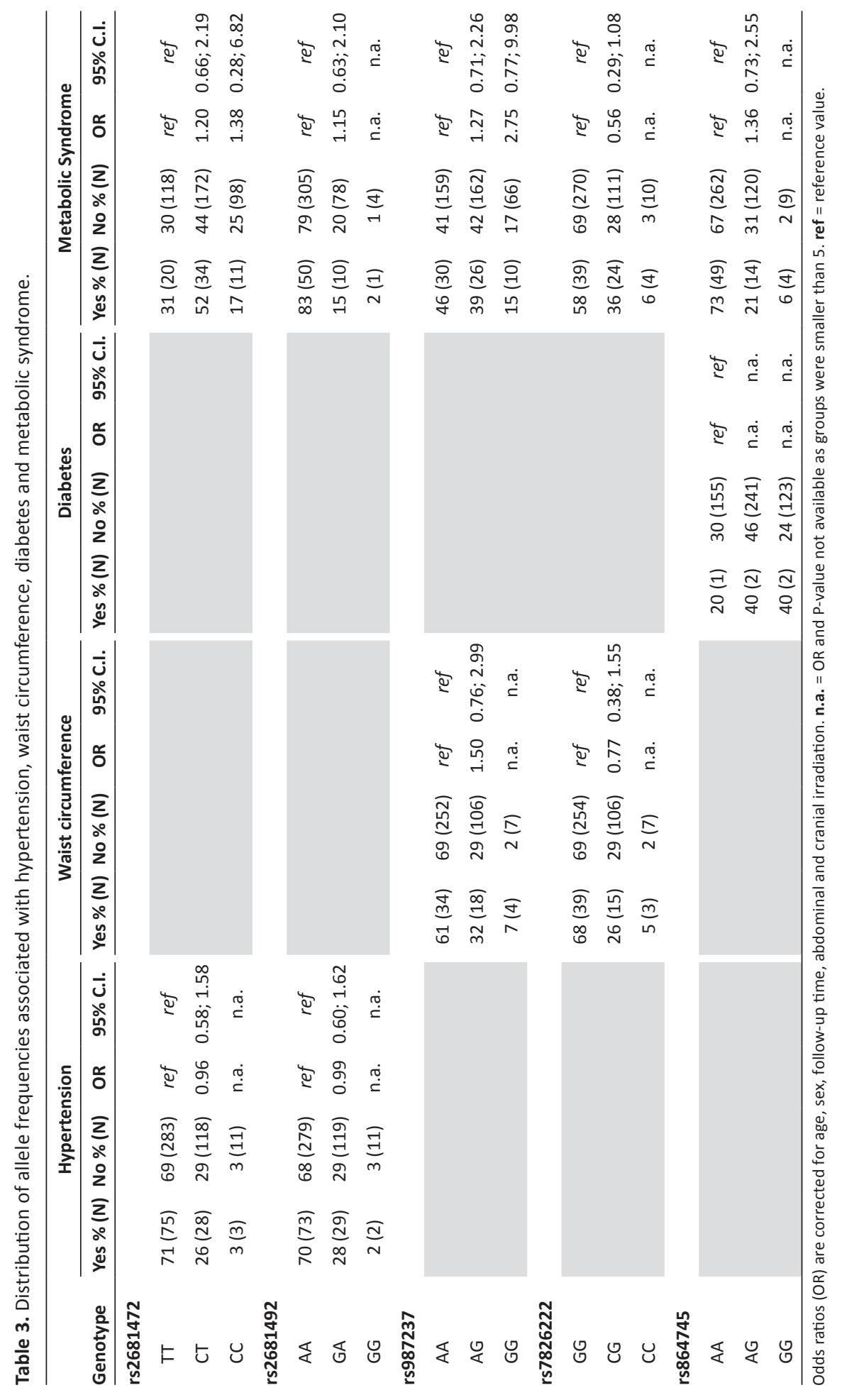




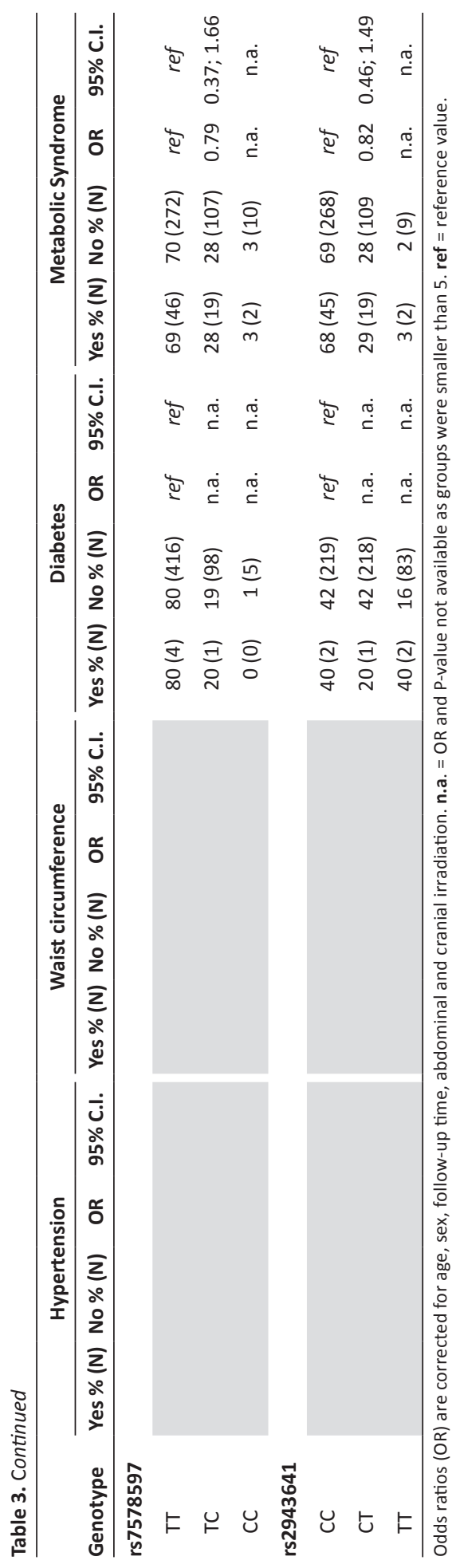




\section{Discussion}

The present study describes the influence of genetic variation on (components of) the metabolic syndrome in a single centre cohort of 532 adult long-term childhood cancer survivors. We found cranial and abdominal radiotherapy to be strongly associated with the occurrence of the (components of the) metabolic syndrome. No association of the candidate SNPs (genes JAZF1, THADA, IRS1, MRSA, TFAP2B, ATP2B1) ${ }^{10,13,15,18}$ with diabetes, waist circumference and blood pressure was observed. Furthermore, none of the seven SNPs was associated with the complete picture of the metabolic syndrome. This suggests that treatment factors, in particular cranial or abdominal radiotherapy, are a far more important for the development of metabolic syndrome in childhood cancer survivors than genetic variation is. Our study was underpowered for SNPs with low allelic frequency, which stresses the fact that genetic variation needs to be studied in larger cohorts. Although to date many studies reported on late effects in childhood cancer survivors, genetic variation studies in large cohorts are scarce. Szymon et al. found that homozygosity of the rs9939609 allele of the FTO-gene in possibly had a protective effect on becoming overweight $191 \mathrm{ALL}$ survivors. ${ }^{21}$ SNPs associated with the leptin and leptin receptor gene were not associated with overweight in 82 ALL survivors. ${ }^{22}$ SNPs in the estrogen receptor marker have been associated with azoospermia in 143 male childhood cancer survivors ${ }^{23}$ and SNPs associated with cardiac damage after anthracyclines treatment have been reported in 76 long-term ALL survivors. ${ }^{24}$ Folate pathway SNPs related to attention disorders in 48 childhood leukemia survivors have also been reported..$^{25}$ These findings do suggest that genetic variation might play a role in development of late effects in childhood cancer survivors.

Although it is very unlikely that SNPs have a stronger influence than treatment factors on development of (components of the metabolic syndrome) in childhood cancer survivors, it is possible that SNPs can be included in a predictive model for metabolic syndrome also involving lifestyle factors and treatment components. However this can only be established in large international collaborative studies with sufficient power. Finally, future GWAS might identify additional alleles associated with hypertension, central adiposity and insulin resistance, although the probability for finding SNPs with a stronger association than the already established SNPs $^{10,12,13,15}$ is not very high.

In summary, we found no associations between rs864745 (JAZF1 gene), rs7578597 (THADA gene), rs2943641 (IRS1 gene), rs987237 (TFAP2B gene), rs7826222 (MSRA gene), rs2681472 and rs2681492 (both ATP2B1 gene) with type 2 diabetes, waist circumference, hypertension and prevalence of metabolic syndrome, suggesting that treatment factors are more important than these candidate genes for determining metabolic syndrome in childhood cancer survivors. 


\section{Supplementary tables}

Table 1. Frequency of (components of) the metabolic syndrome in diagnosis subgroups $(\mathrm{N}>30)$.

\begin{tabular}{|c|c|c|}
\hline & Diagnosis subgroup & P-value ${ }^{a}$ \\
\hline \multirow[t]{8}{*}{ Diabetes } & ALL & 1.000 \\
\hline & B-NHL & 1.000 \\
\hline & Hodgkin & 1.000 \\
\hline & Sarcoma & 1.000 \\
\hline & Wilms & 0.002 (5.5\% vs. $0.2 \%)$ \\
\hline & NBL & 1.000 \\
\hline & MMT & 1.000 \\
\hline & Brain tumor & 1.000 \\
\hline \multirow[t]{8}{*}{ Obesitas } & ALL & 0.194 \\
\hline & B-NHL & 0.015 (0.0\% vs. $10.0 \%)$ \\
\hline & Hodgkin & 0.767 \\
\hline & Sarcoma & 0.153 \\
\hline & Wilms & 0.541 \\
\hline & NBL & 1.000 \\
\hline & MMT & 0.571 \\
\hline & Brain tumor & 0.293 \\
\hline \multirow[t]{8}{*}{ High waist circumference } & ALL & 0.065 \\
\hline & $\mathrm{B}-\mathrm{NHL}$ & 0.202 \\
\hline & Hodgkin & 0.268 \\
\hline & Sarcoma & 0.149 \\
\hline & Wilms & 0.130 \\
\hline & NBL & 0.342 \\
\hline & MMT & 1.000 \\
\hline & Brain tumor & $0.030(29.2 \%$ vs. $12.5 \%)$ \\
\hline \multirow[t]{7}{*}{ High total cholesterol } & ALL & $0.026(29.1 \%$ vs. $39.0 \%)$ \\
\hline & B-NHL & 0.746 \\
\hline & Hodgkin & 0.885 \\
\hline & Sarcoma & 0.292 \\
\hline & Wilms & 0.280 \\
\hline & NBL & 0.311 \\
\hline & MMT & 0.560 \\
\hline
\end{tabular}

${ }^{\mathrm{a} A s}$ compared with other diagnosis subgroups. 
Table 1. Continued

\begin{tabular}{lll}
\hline & Diagnosis subgroup & P-value $^{\text {a }}$ \\
\hline Bypertension & Brain tumor & 0.015 (58.6\% vs. 34.4\%) \\
ALL & 0.979 \\
B-NHL & 0.189 \\
Hodgkin & 0.591 \\
Sarcoma & 1.000 \\
Wilms & 0.663 \\
NBL & 0.061 \\
MMT & 0.074 \\
Brain tumor & 0.370 \\
ALL & 0.900 \\
B-NHL & 0.425 \\
Hodgkin & 1.000 \\
Sarcoma & 0.334 \\
Wilms & 0.522 \\
NBL & 0.879 \\
MMT & 0.387 \\
Brain tumor & 0.259 \\
\hline
\end{tabular}

${ }^{a}$ As compared with other diagnosis subgroups.

Table 2. Frequency of (components of ) the metabolic syndrome in radiotherapy subgroups.

\begin{tabular}{lll}
\hline & Therapy subgroup & P-value $^{\text {a }}$ \\
\hline Diabetes & Cranial irradiation & 1.000 \\
Obesitas & Abdominal irradiation & 0.007 (6.1\% vs. $0.3 \%)$ \\
High waist & Cranial irradiation & 0.074 \\
& Abdominal irradiation & 1.000 \\
High total cholesterol & Cranial irradiation & $0.002(25.0 \%$ vs. $10.5 \%)$ \\
Hypertension & Abdominal irradiation & 0.279 \\
Metabolic syndrome & Cranial irradiation & $<0.001(53.5 \%$ vs. $27.1 \%)$ \\
& Abdominal irradiation & $<0.001(54.2 \%$ vs. $27.1 \%)$ \\
& Cranial irradiation & 0.293 \\
\hline
\end{tabular}

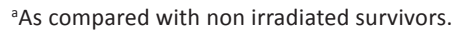




\section{References}

1. van Waas M, Neggers SJ, Pieters R, van den Heuvel-Eibrink MM. Components of the metabolic syndrome in 500 adult long-term survivors of childhood cancer. Ann Oncol 2010;21:1121-6.

2. van Waas $M$, Neggers SJ, Te Winkel ML, Beishuizen A, Pieters R, van den Heuvel-Eibrink MM. Endocrine late sequelae in long-term survivors of childhood non-Hodgkin lymphoma. Ann Oncol 2011 Jun;23(6):1626-32.

3. van Waas M, Neggers SJ, van der Lelij AJ, Pieters R, van den Heuvel-Eibrink MM. The metabolic syndrome in adult survivors of childhood cancer, a review. J Pediatr Hematol Oncol 2010;32:171-9.

4. Chow EJ, Simmons JH, Roth CL, et al. Increased cardiometabolic traits in pediatric survivors of acute lymphoblastic leukemia treated with total body irradiation. Biol Blood Marrow Transplant 2010 Dec;16(12):1674-81

5. Oeffinger KC, Buchanan GR, Eshelman DA, et al. Cardiovascular risk factors in young adult survivors of childhood acute lymphoblastic leukemia. J Pediatr Hematol Oncol 2001;23:424-30.

6. Meacham LR, Chow EJ, Ness KK, et al. Cardiovascular risk factors in adult survivors of pediatric cancer--a report from the childhood cancer survivor study. Cancer Epidemiol Biomarkers Prev 2010 Jan;19(1):170-81.

7. Executive Summary of The Third Report of The National Cholesterol Education Program (NCEP) Expert Panel on Detection, Evaluation, And Treatment of High Blood Cholesterol In Adults (Adult Treatment Panel III). JAMA 2001;285:2486-97.

8. Manolio TA, Brooks LD, Collins FS. A HapMap harvest of insights into the genetics of common disease. J Clin Invest 2008;118:1590-605.

9. McCarthy MI, Hirschhorn JN. Genome-wide association studies: potential next steps on a genetic journey. Hum Mol Genet 2008;17:R156-65.

10. Levy D, Ehret GB, Rice K, et al. Genome-wide association study of blood pressure and hypertension. Nat Genet 2009;41:677-87.

11. Pande J, Mallhi KK, Sawh A, Szewczyk MM, Simpson F, Grover AK. Aortic smooth muscle and endothelial plasma membrane Ca2+ pump isoforms are inhibited differently by the extracellular inhibitor caloxin 1b1. Am J Physiol Cell Physiol 2006;290:C1341-9.

12. Lindgren CM, Heid IM, Randall JC, et al. Genome-wide association scan meta-analysis identifies three Loci influencing adiposity and fat distribution. PLoS Genet 2009;5:e1000508.

13. Rung J, Cauchi S, Albrechtsen A, et al. Genetic variant near IRS1 is associated with type 2 diabetes, insulin resistance and hyperinsulinemia. Nat Genet 2009;41:1110-5.

14. Grarup N, Andersen G, Krarup NT, et al. Association testing of novel type 2 diabetes risk alleles in the JAZF1, CDC123/CAMK1D, TSPAN8, THADA, ADAMTS9, and NOTCH2 loci with insulin release, insulin sensitivity, and obesity in a population-based sample of 4,516 glucose-tolerant middle-aged Danes. Diabetes 2008;57:2534-40.

15. Zeggini E, Scott LJ, Saxena R, et al. Meta-analysis of genome-wide association data and large-scale replication identifies additional susceptibility loci for type 2 diabetes. Nat Genet 2008;40:638-45.

16. Haugnes HS, Aass N, Fossa SD, et al. Components of the metabolic syndrome in long-term survivors of testicular cancer. Ann Oncol 2007;18:241-8.

17. Lindgren CM, Heid IM, Randall JC, et al. Genome-wide association scan meta-analysis identifies three Loci influencing adiposity and fat distribution. PLoS Genet 2009;5:e1000508.

18. Zeggini E, Scott LJ, Saxena R, et al. Meta-analysis of genome-wide association data and large-scale replication identifies additional susceptibility loci for type 2 diabetes. Nat Genet 2008;40:638-45. 
19. Stolk L, Zhai G, van Meurs JB, et al. Loci at chromosomes 13, 19 and 20 influence age at natural menopause. Nat Genet 2009;41:645-7.

20. Szymon S, Bik-Multanowski M, Balwierz W, et al. Homozygosity for the rs9939609T allele of the FTO gene may have protective effect on becoming overweight in survivors of childhood acute lymphoblastic leukemia. J Genet 2011 Aug;90(2):365-8.

21. Skoczen S, Tomasik PJ, Bik-Multanowski M, et al. Plasma levels of leptin and soluble leptin receptor and polymorphisms of leptin gene -18G > A and leptin receptor genes K109R and Q223R, in survivors of childhood acute lymphoblastic leukemia. J Exp Clin Cancer Res 2011 Jun 1;30:64.

22. Romerius P, Giwercman A, Moell C, et al. Estrogen receptor alpha single nucleotide polymorphism modifies the risk of azoospermia in childhood cancer survivors. Pharmacogenet Genomics 2011 May;21(5):263-9.

23. Rajic V, Aplenc R, Debeljak M, et al. Influence of the polymorphism in candidate genes on late cardiac damage in patients treated due to acute leukemia in childhood. Leuk Lymphoma 2009;50:1693-8.

24. Krull KR, Brouwers $\mathrm{P}$, Jain $\mathrm{N}$, et al. Folate pathway genetic polymorphisms are related to attention disorders in childhood leukemia survivors. J Pediatr 2008;152:101-5. 

Due to the improving survival rates of childhood cancer, to date an increasing number of young adults is a survivor of childhood cancer. As mortality rates in childhood cancer survivors exceed those of the normal population, late effects of childhood cancer have increasingly gained attention. ${ }^{1}$ Metabolic syndrome and, consequently, an increased risk for cardiovascular diseases, seem to be a significant part of the burden of disease experienced by childhood cancer survivors. The first large group of childhood cancer survivors is now entering the period of life when premature cardiovascular events start to occur. ${ }^{2}$ Given their increased risk for cardiovascular diseases, this emphasizes the clinical relevance of the studies presented in this thesis.

In the present chapter we will discuss the main findings of the reported studies in the context of the literature. We will emphasize the implications of our findings and make suggestions about future research.

The specific aims of the research presented in this thesis were:

1. To determine the frequency of the metabolic syndrome in adult survivors of childhood cancer.

2. To identify which risk factors, especially treatment factors, increase the risk for metabolic syndrome after childhood cancer.

3. To identify additional cardiovascular risk factors associated with the metabolic syndrome in childhood cancer survivors.

4. To identify the influence of genetic variation on the development of the metabolic syndrome in childhood cancer survivors.

\subsection{Frequency of the metabolic syndrome}

The first aim of this thesis was to assess the frequency of the metabolic syndrome in childhood cancer survivors. We found the frequency in the total group of survivors to be comparable to that of the normal population ${ }^{3}$ (chapter three). This suggests that survivors of childhood cancer are not at increased risk for development of the metabolic syndrome. However, although the frequency of the metabolic syndrome was not higher, survivors had higher systolic blood pressure and serum total cholesterol levels. This indicates that when assessing the metabolic syndrome, important information can be lost. The dichotomization of the metabolic syndrome may consequently lead to an incorrect interpretation of the risk for cardiovascular diseases. We therefore additionally studied continuous variables in our prospective studies, which provide more information in research settings. Furthermore, we showed that although the overall frequency of the metabolic syndrome was comparable to that of the normal population, it was increased in survivors treated with cranial irradiation. This is described in more detail in paragraph 10.2 of this chapter. Finally, due to the fact that we had no fasting blood samples in these survivors, we were not able to assess the dyslipidemia component of the regular metabolic 
syndrome definitions..$^{4-9}$ We therefore used a modified definition, ${ }^{10}$ which showed to be useful when performing subgroup analyses, but makes statistical comparison of the frequency of the metabolic syndrome between our survivors and the normal population difficult. Based on the literature and our retrospective study we therefore use fasting laboratory parameters in our prospective studies to assess the metabolic syndrome according to international standardized criteria..$^{4-9}$

\subsection{Risk factors for the metablic syndrome}

The second aim of the research described in this thesis was to identify which risk factors, especially treatment factors, increase the risk for metabolic syndrome after childhood cancer. We first focused on survivors of haematological malignancies: i.e. acute lymphoblastic leukemia (ALL) and non-Hodgkin lymphoma (NHL) survivors.

Because of the relatively high incidence and survival rate, ${ }^{2}$ the majority of reports on late effects in childhood cancer survivors has focused on ALL survivors. Comparable with other studies, ${ }^{10-12}$ we observed ALL survivors treated with cranial irradiation to be at higher risk for the metabolic syndrome than ALL survivors not treated with cranial irradiation (chapter two). In addition, survivors treated with cranial irradiation were more often overweight and had lower insulin like growth factor (IGF)-1 levels, which is indicative for growth hormone deficiency. Low IGF-1 levels have been associated with an increased risk for metabolic syndrome, ${ }^{13}$ cardiovascular diseases and atherosclerosis, ${ }^{14}$ hyperlipidemia, ${ }^{15}$ coronary heart disease, ${ }^{16}$ fatty liver, ${ }^{17}$ and a proinflammatory state. ${ }^{18}$ In ALL survivors treated with cranial irradiation, damage to the hypothalamic-pituitary axis is largely responsible for the long-term effects of growth hormone deficiency. Growth hormone deficiency is a potentially atherogenic state, likely related, at least in part, to its association with low IGF-1 levels. ${ }^{19}$ The role of cranial irradiation in the development of metabolic syndrome is now well established. Future studies in ALL survivors should focus on prevention and treatment, in order to prevent the occurrence of cardiovascular events. Growth hormone deficiency treatment has been shown to have beneficial effects on metabolic syndrome, intima media thickness, insulin sensitivity, glucose tolerance, anthropometric parameters, and IGF-1 and IGFBP-3 in children born for gestational age and adults with growth hormone deficiency. ${ }^{20-23}$ However, the positive effects of growth hormone replacement while on therapy usually do not persist after growth hormone is discontinued. ${ }^{19} \mathrm{~A}$ small study in eleven ALL survivors showed that growth hormone replacement therapy led to positive effects on body composition, but hyperleptinemia, hyperinsulinemia and impaired insulin sensitivity remained unchanged. ${ }^{24}$ Whether growth hormone treatment improves the metabolic and cardiovascular risk profile in childhood cancer survivors needs to be evaluated in large, preferably randomized controlled trials. Ideally, these trials should include three study arms: one with placebo treatment, one with short-term growth hormone treatment and one with long-term growth hormone treatment. 
Because ALL survivors are known to be at increased risk for obesity and disturbed body composition. ${ }^{2,11,25,26}$ and treatment regimens for lymphoblastic NHL are similar to those for ALL, we studied NHL survivors to see whether they are also at higher risk for altered body composition or decreased bone mineral density. Body composition and bone mineral density, both measured with a dual-energy X-ray absorptiometry (DXA)-scan, were normal in NHL survivors. Thyroid function which may influence obesity and body composition, ${ }^{27}$ was normal in NHL survivors. Because of the known link between obesity and infertility, ${ }^{28}$ we also studied fertility markers in this subgroup of survivors but found no association between fertility markers and obesity. However, inhibin B, a marker for male fertility, appeared to be lower in male survivors (chapter three). The risk factors for impairment of fertility in childhood cancer survivors are age at time of treatment, chemotherapy with alkylating agents and irradiation of the reproductive organs or the hypothalamus/pituitary. ${ }^{29}$ Interestingly, in our study cytarabine was associated with lower inhibin B levels in males. This has not been reported previously in childhood cancer survivors, but an association between cytarabine and gonadal damage in survivors of adult acute myeloid has been observed..$^{30}$ The exact mechanism behind this pathway is yet unknown. Future studies are warranted to evaluate the influence of cytarabine, preferably in large cohorts enabling disentanglement of the other above-mentioned risk factors.

In clinical practice, NHL survivors may be counseled concerning their risk for impaired fertility. Research is currently focusing on new techniques enabling fertility preservation. ${ }^{31}$ This may lead to new possibilities for patients to preserve their possibility to have children.

In contrast to studies in ALL survivors, studies in survivors of solid tumors, for example nephroblastoma and neuroblastoma survivors, are scarce. We therefore consequently focused on survivors of nephroblastoma and neuroblastoma. Before analyzing the metabolic syndrome in nephroblastoma and neuroblastoma survivors, we studied three important confounders. First, we observed that all nephroblastoma and neuroblastoma survivors, regardless of the presence of both or one adrenal gland(s), had adequate adrenal function (chapter five). Interestingly, survivors treated with unilateral adrenalectomy had a relatively high central setpoint of the hypothalamicpituitary-adrenal axis characterized by high basal cortisol and adrenocorticotrophin hormone (ACTH). Raised cortisol levels have been previously reported in childhood cancer survivors after cranial irradiation. ${ }^{32}$ This could either be explained by damage of the hypothalamus/pituitary gland, or by physical/psychosocial stress. However, if the latter would be the explanation, similar cortisol and ACTH levels would have been observed in survivors treated without adrenalectomy. The hypothesis of a higher 'setpoint' of the adrenal system in subjects after adrenalectomy is therefore more reasonable. Our results thus suggest that the feedback system has been altered, which might be due to a feedback loop of the neural pathways, damaged during surgery. Such mechanism has been described for the thyroid gland, which has neural connections with the hypothalamic suprachiasmatic and paraventricular nuclei. ${ }^{33}$ The thyroid hormone affects target tissues directly via thyroid hormone receptors, but also indirectly through effects on the integration of the sympathetic signal in target tissues, and possibly also via the pre-autonomic 
neurones in the central nervous system. ${ }^{34}$ It is possible that cortisol acts in a way similar to the thyroid hormone. In our subset of adrenalectomized survivors, basal cortisol and ACTH levels were associated with dyslipidemia and insulin resistance, highlighting the relevance of this finding concerning the risk for metabolic syndrome and cardiovascular diseases in these survivors. Future studies are warranted to unravel the exact mechanism of the high central hypothalamic-pituitary-adrenal axis setpoint after adrenalectomy. Rodent or mice studies, following an approach similar to that performed for thyroid hormone ${ }^{34,35}$ may shed light on this complicated pathway.

The second confounder was the reduced physical activity in male neuroblastoma survivors, which was not due to cardiomyopathy or spinal deformities (chapter six). However, it needs to be considered that the group of male neuroblastoma survivors consisted of only 15 subjects. Results should therefore be interpreted with caution. However, as reduced physical activity is known to be associated with the metabolic syndrome, ${ }^{36}$ we adjusted for physical activity in our studies where necessary. Ideally, studies involving physical activity should use objective measurements such as heart rate telemetry, pedometry and accelerometry. ${ }^{37}$

Thirdly, we stressed the fact that prevalence of the metabolic syndrome in survivors treated with abdominal irradiation may be underestimated when international standardized criteria are used, due to their dysmorphic abdominal area. This was illustrated by the fact that although waist circumference was indeed lower in irradiated survivors, total percentage fat, as measured by DXA-scan, was significantly higher (chapter seven). To our knowledge, we are the first to report on this relevant topic and we think that this finding should be considered when studying the metabolic syndrome in abdominally irradiated survivors. Although it is expensive and time consuming, studies involving computerised tomography (CT) or Magnetic Resonance Imaging (MRI) scan, which can provide the most precise estimate of the location and amount of adipose tissue in various body regions $\mathrm{s}^{38}$ might provide information on the true contribution of abdominal adiposity to cardiovascular risk in abdominally irradiated survivors. For clinical practice we recommend assessing alternative markers for adiposity in irradiated survivors, for example total percentage fat as measured by DXA-scan, which is more feasible and less expensive than the previously mentioned $\mathrm{CT}$ and MR-scans.

When these important confounders were taken into account, we observed that the metabolic syndrome was three times more frequent in survivors treated with abdominal irradiation than in survivors not treated with irradiation (chapter seven). Furthermore, although the pancreas has always been considered as relatively insensitive to radiation, ${ }^{39,40}$ we showed that radiotherapy involving both head and tail of the pancreas influenced the occurrence of dyslipidemia and insulin resistance. Radiation induced apoptosis of pancreatic beta cells may play a causal role in this. It may lead to decreased insulin production which induces hyperglycemia, elevated free fatty acids (FFA) levels and hypertriglyceridemia. This may eventually lead to increased insulin resistance. We also showed that abdominal irradiation involving the complete liver influences dyslipidemia, in contrast to irradiation involving only part of the liver. These results indicate that 
non-irradiated liver and non-irradiated pancreas fields may have the capacity to compensate for impaired liver and pancreas function of the irradiated fields. Previous studies have shown an association between abdominal radiotherapy and components of the metabolic syndrome and cardiovascular risk, ${ }^{41-43}$ however, detailed information on which organs were included in the radiation field was not reported.

Furthermore, chemotherapeutic agents might damage the vascular endothelium, resulting in vascular alterations which may lead to the reported hypertension and dyslidemia. Studies involving radiation dosimetry and cumulative doses of specific chemotherapeutic agents are warranted to elucidate the exact mechanism behind the development of metabolic syndrome. To disentangle the separate effects of treatment components, these effects should be studied in large cohorts of survivors. In clinical practice, doctors should be aware of the increased risk for metabolic syndrome in survivors treated with abdominal irradiation and, to a less extent, chemotherapy. Currently, in the Netherlands, screening of survivors is considered to be secondary care, performed by pediatric oncologists (in children) or by internists (in adults). Follow-up is based on the Dutch guidelines (ISBN: 9789079691036) for follow-up of childhood cancer as developed by the Dutch Children Oncology Group. Screening for the components of the metabolic syndrome may lead to early recognition of risk factors. Although there is currently no drug therapy to treat metabolic syndrome as a whole disease, improvements in diet and lifestyle or, secondary, treatment of individual components, may reduce the risk for metabolic syndrome and consequently cardiovascular diseases in childhood cancer survivors. ${ }^{44}$

Finally, nephrectomy and adrenalectomy seemed to have beneficial effects concerning dyslipidemia and insulin resistance and thus do not seem to increase the risk for development of the metabolic syndrome. However, although there might not be a direct effect of nephrectomy and adrenelectomy, indirect pathways might still increase the risk for metabolic syndrome. This is illustrated by the fact that high basal cortisol levels, induced by adrenalectomy, are associated with dyslipidemia and insulin resistance (chapter four).

\subsection{Additional cardiovascular risk factors}

The third aim of this thesis was to identify additional cardiovascular risk factors associated with the metabolic syndrome in childhood cancer survivors. Classic markers for cardiovascular risk are low density lipoprotein-cholesterol (LDL-C), smoking behavior, hypertension and body mass index. The relationship between the metabolic syndrome, hypertension, adiposity, dyslipidemia and insulin resistance with cardiovascular diseases is well established. ${ }^{4-9,44}$ Several other biomarkers, such as proinflammatory and prothrombotic markers, have been associated with cardiovascular risk. ${ }^{45-49}$ According to a recent review, a combination of biomarkers reflecting different pathophysiologic pathways will be most useful in yielding incremental information above and beyond that gained by using traditional parameters. ${ }^{50}$ We therefore applied principal 
component analysis, enabling us to take into account the combined effects of a variety of biomarkers. Furthermore, we studied relatively new identified markers that provide information on the condition of the vascular system, such as central blood pressure, intima media thickness and pulse wave velocity. ${ }^{51-55}$

We observed survivors treated with abdominal irradiation to have risk profiles characterized by adverse lipids and by plasminogen activator inhibitor 1 (PAI-1) and FFA, increased blood pressure and features of arterial stiffness (chapter eight). Together with the increased total body fat percentage as reported in chapter seven, abdominally irradiated survivors thus face an increased cardiovascular risk. As described in chapter one, secondary effects of increased adiposity include increased circulating FFA (in part resulting from a net increase in lipolysis), reduced adiponectin, a potential increase in insulin resistance factors, and increased proinflammatory mediators. ${ }^{56}$ Although insulin resistance is the perceived pathophysiology of the metabolic syndrome, we did not find abdominally irradiated survivors to be more insulin resistant, neither did they express a proinflammatory state. However, as these survivors already express adiposity, dyslipidemia, high blood pressure and features of arterial stiffness, it is likely that they are at increased risk for developing insulin resistance and possibly type 2 diabetes.

Survivors with a history of nephrectomy were at risk for impaired renal function, as characterized by cystatin C, uric acid, urea and homocystein (chapter eight). Directly or indirectly via impaired renal function, these markers have been associated with a higher risk for cardiovascular disease. ${ }^{45-47}$ Although in chapter seven nephrectomy appeared to be associated with a favourable lipid profile, the question remains whether decreased renal function influences development of dyslipidemia and other components of the metabolic syndrome. The direct effect of impaired renal function on components of the metabolic syndrome would provide additional information on cardiovascular risk in nephroblastoma and neuroblastoma survivors. Furthermore, cumulative dose of actinomycin and cyclophosphamide were associated with features of arterial stiffness (chapter eight). As we also observed chemotherapy to be associated with hypertension and dyslipidemia (chapter seven), these components probably play a causal role in the development of arterial stiffness.

Although we included environmental factors such as age, sex, smoking, physical activity and socio-economic status in our analyses, we were unfortunately not able to assess the influence of diet. However, nephroblastoma and neuroblastoma survivors are not treated with cranial irradiation and thus do not seem to be at risk for hypothalamic-pituitary damage resulting in altered dietary patterns. It seems therefore unlikely that diet of these survivors is different from that of their controls.

In clinical practice, doctors should be aware that combined effects of several treatment risk factors may lead to substantial risk for cardiovascular disease. Furthermore, we have to realise that risk factors often occur in clusters and that consequently, when one risk factor is established, it might well be that more risk factors are present. Survivors expressing clusters of high PAI-1 and FFA levels will probably benefit from the previously mentioned improvements in 
diet and lifestyle or, secondary, treatment of insulin resistance. Similarly, arterial stiffness might be treated with changes in lifestyle behaviour or with treatment of blood pressure. Among the antihypertensive agents that may offer useful tools to lower pulse pressure and arterial stiffness are nitrates, nitric oxide donors and drugs that interfere with the renin-angiotensin-aldosterone. ${ }^{57}$ Future developments may include non-antihypertensive agents that target collagen or other components of the arterial wall matrix. However, large-scale clinical trials will have to confirm the therapeutic value of these agents in the treatment of increased pulse pressure and arterial stiffness. ${ }^{57}$

\subsection{Genetic variation and metabolic syndrome}

The metabolic syndrome is, in the general population, known to be determined by genetic makeup. The fourth aim of the study was therefore to evaluate influence of genetic variation based on candidate single nucleotide polymorphisms (SNPs) in genes on the development of the metabolic syndrome in childhood cancer survivors. We found no association of the candidate SNPs in the genes JAZF1, THADA, IRS1, MRSA, TFAP2B, ATP2B1 $)^{58-61}$ with diabetes, waist circumference and blood pressure. Furthermore, none of the seven SNPs was associated with the complete picture of the metabolic syndrome. Although our study was underpowered for SNPs with low allelic frequency, this suggests that treatment components are far more important for the development of metabolic syndrome in childhood cancer survivors than genetic variation is (chapter nine). It therefore seems unlikely that childhood cancer survivors carry more disadvantageous SNPS related to (components of the) metabolic syndrome than the normal population.

\subsection{This thesis as a pilot late effects study}

Currently, several large organisations collaborate in order to investigate late effects in childhood cancer survivors. Examples are the Childhood Cancer Survivor Study (CCSS), British Childhood Cancer Survivors Study (BCCSS) and Pan-European Network for Care of Survivors after Childhood Cancer (PanCare).

In 2012 a large nationwide study, called Dutch Childhood Oncology Group (DCOG) - LATER Q2008, will start in 7000 long-term survivors in the Netherlands. Besides the metabolic syndrome and associated cardiovascular risk factors, many other late effects will be assessed including endocrine late effects, secondary malignancies, cardiotoxicity, infertility, neurocognitive and psychosocial late effects. The study will have enough power to assess late effects such as the metabolic syndrome in specific subgroups of childhood cancer survivors. Q2008 is a unique late effects study as it will actively recruit participants and will assess all outcome parameters directly, whilst many other studies use questionnaires to assess late effects. 


\subsection{Conclusions}

The results described in this thesis are summarized in Figure 1. We found the frequency of childhood cancer survivors as a group to be comparable to that in the general population.

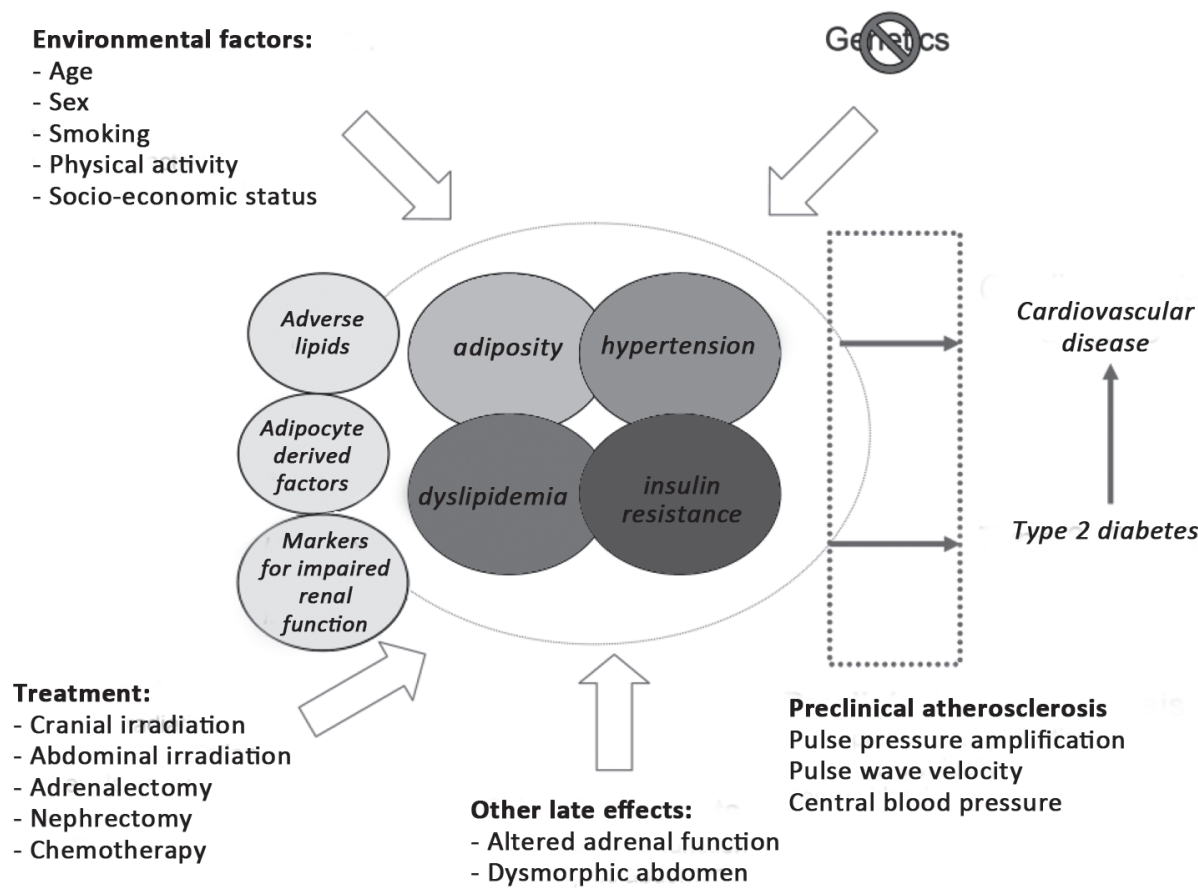

Figure 1. Summary of the results described in this thesis.

Cranial and abdominal radiotherapy emerged as the strongest determinants of metabolic syndrome and cardiovascular risk. Furthermore, our results suggested that non-irradiated liver and non-irradiated pancreas fields may have the capacity to compensate for impaired liver and pancreas function of the irradiated fields. Nephrectomy, adrenalectomy and chemotherapy also seemed to be associated with features of the metabolic syndrome and cardiovascular risk profiles, however the direct pathophysiological mechanisms remain to be elucidated. Features of preclinical atherosclerosis were already present in specific subgroups of survivors. Furthermore, we identified clusters of cardiovascular risk factors which may yield incremental information above and beyond that of single risk factors.

We identified late effects, i.e. altered adrenal function and dysmorphic abdomen due to irradiation and surgery, as important confounders for the metabolic syndrome and showed that the metabolic syndrome may be underestimated when using standard variables according to the 
NCEP criteria. Furthermore, we observed male NHL survivors to be at increased risk for impaired fertility. An effect of genetic variation on the metabolic syndrome was not established. Finally, we adjusted for the influence of environmental factors to prevent confounding.

At this point the cohort of survivors is too young to measure clinical endpoints such as myocardial infarction or death. It is however clear that, as relatively young childhood cancer survivors already express components of the metabolic syndrome and other cardiovascular risk factors, childhood cancer survivors face an increased risk for cardiovascular diseases. 


\section{References}

1. Mertens AC, Liu Q, Neglia JP, et al. Cause-specific late mortality among 5-year survivors of childhood cancer: the Childhood Cancer Survivor Study. J Natl Cancer Inst 2008;100:1368-79.

2. Oeffinger KC. Are survivors of acute lymphoblastic leukemia (ALL) at increased risk of cardiovascular disease? Pediatr Blood Cancer 2008;50:462-7.

3. Bos MB, de Vries JH, Wolffenbuttel BH, Verhagen $\mathrm{H}$, Hillege JL, Feskens EJ. [The prevalence of the metabolic syndrome in the Netherlands: increased risk of cardiovascular diseases and diabetes mellitus type 2 in one quarter of persons under 60]. De prevalentie van het metabool syndroom in Nederland: verhoogd risico op hart- en vaatziekten en diabetes mellitus type 2 bij een kwart van de personen jonger dan 60 jaar. Ned Tijdschr Geneeskd 2007;151:2382-8.

4. Alberti KG, Zimmet P, Shaw J. The metabolic syndrome--a new worldwide definition. Lancet 2005;366:1059-62.

5. Alberti KG, Zimmet P, Shaw J. Metabolic syndrome--a new world-wide definition. A Consensus Statement from the International Diabetes Federation. Diabet Med 2006;23:469-80.

6. Balkau B, Charles MA, Drivsholm T, et al. Frequency of the WHO metabolic syndrome in European cohorts, and an alternative definition of an insulin resistance syndrome. Diabetes \& metabolism 2002;28:364-76.

7. Einhorn D, Reaven GM, Cobin RH, et al. American College of Endocrinology position statement on the insulin resistance syndrome. Endocr Pract 2003;9:237-52.

8. Grundy SM, Becker D, Clark LT, et al. Executive Summary of The Third Report of The National Cholesterol Education Program (NCEP) Expert Panel on Detection, Evaluation, And Treatment of High Blood Cholesterol In Adults (Adult Treatment Panel III). JAMA 2001;285:2486-97.

9. Grundy SM, Cleeman JI, Daniels SR, et al. Diagnosis and management of the metabolic syndrome: an American Heart Association/National Heart, Lung, and Blood Institute Scientific Statement. Circulation 2005;112:2735-52.

10. Haugnes HS, Aass N, Fossa SD, et al. Components of the metabolic syndrome in long-term survivors of testicular cancer. Ann Oncol 2007;18:241-8.

11. Aldhafiri F, Al-Nasser A, Al-Sugair A, Al-Mutairi H, Young D, Reilly JJ. Obesity and metabolic syndrome in adolescent survivors of standard risk childhood acute lymphoblastic leukemia in Saudi Arabia. Pediatr Blood Cancer 2011.

12. Gurney JG, Ness KK, Sibley SD, et al. Metabolic syndrome and growth hormone deficiency in adult survivors of childhood acute lymphoblastic leukemia. Cancer 2006;107:1303-12.

13. Lam CS, Chen MH, Lacey SM, et al. Circulating insulin-like growth factor-1 and its binding protein-3: metabolic and genetic correlates in the community. Arterioscler Thromb Vasc Biol 2010;30:1479-84.

14. Arai Y, Kojima T, Takayama M, Hirose N. The metabolic syndrome, IGF-1, and insulin action. Mol Cell Endocrinol 2009;299:124-8.

15. Malik J, Stulc T, Wichterle D, et al. Hyperlipidemia is associated with altered levels of insulin-like growth factor-I. Physiol Res 2008;57:919-25.

16. Akanji AO, Suresh CG, Al-Radwan R, Fatania HR. Insulin-like growth factor (IGF)-I, IGF-II and IGFbinding protein (IGFBP)-3 levels in Arab subjects with coronary heart disease. Scand J Clin Lab Invest 2007;67:553-9.

17. Volzke H, Nauck M, Rettig R, et al. Association between hepatic steatosis and serum IGF1 and IGFBP-3 levels in a population-based sample. Eur J Endocrinol 2009;161:705-13.

18. Efstratiadis G, Tsiaousis G, Athyros VG, et al. Total serum insulin-like growth factor-1 and C-reactive protein in metabolic syndrome with or without diabetes. Angiology 2006;57:303-11.

19. Akanji AO, Smith RJ. The insulin-like growth factor system, metabolic syndrome, and cardiovascular disease risk. Metab Syndr Relat Disord 2012;10:3-13. 
20. Pasarica M, Zachwieja JJ, Dejonge L, Redman S, Smith SR. Effect of growth hormone on body composition and visceral adiposity in middle-aged men with visceral obesity. J Clin Endocrinol Metab 2007;92:4265-70.

21. Di Somma C, Pivonello R, Pizza G, et al. Prevalence of the metabolic syndrome in moderately-severely obese subjects with and without growth hormone deficiency. J Endocrinol Invest 2010;33:171-7.

22. Colao A, Di Somma C, Spiezia S, et al. Growth hormone treatment on atherosclerosis: results of a 5-year open, prospective, controlled study in male patients with severe growth hormone deficiency. J Clin Endocrinol Metab 2008;93:3416-24.

23. van der Klaauw AA, Biermasz NR, Feskens EJ, et al. The prevalence of the metabolic syndrome is increased in patients with $\mathrm{GH}$ deficiency, irrespective of long-term substitution with recombinant human GH. Eur J Endocrinol 2007;156:455-62.

24. Bulow B, Link K, Ahren B, Nilsson AS, Erfurth EM. Survivors of childhood acute lymphoblastic leukemia, with radiation-induced $\mathrm{GH}$ deficiency, exhibit hyperleptinemia and impaired insulin sensitivity, unaffected by 12 months of GH treatment. Clin Endocrinol (Oxf) 2004;61:683-91.

25. Veringa SJ, van Dulmen-den Broeder E, Kaspers GJ, Veening MA. Blood pressure and body composition in long-term survivors of childhood acute lymphoblastic leukemia. Pediatr Blood Cancer 2012;58:27882.

26. Skoczen S, Surmiak M, Strojny W. Survivors of acute lymphoblastic leukemia and body mass changes. Expert Opin Drug Saf 2010;9:65-77.

27. Kitahara CM, Platz EA, Ladenson PW, Mondul AM, Menke A, Berrington de Gonzalez A. Body fatness and markers of thyroid function among U.S. men and women. PLoS One;7:e34979.

28. Malnick SD, Knobler H. The medical complications of obesity. QJM 2006;99:565-79.

29. Spix C. Fertility in survivors of childhood cancer. Dtsch Arztebl Int 2012;109:124-5.

30. Lemez P, Urbanek V. Chemotherapy for acute myeloid leukemias with cytosine arabinoside, daunorubicin, etoposide, and mitoxantrone may cause permanent oligoasthenozoospermia or amenorrhea in middle-aged patients. Neoplasma 2005;52:398-401.

31. Lawrenz B, Henes M, Neunhoeffer E, Fehm T, Lang P, Schwarze CP. Fertility preservation in girls and adolescents before chemotherapy and radiation - review of the literature. Klin Padiatr 2011;223:12630.

32. Darzy KH, Shalet SM. Absence of adrenocorticotropin (ACTH) neurosecretory dysfunction but increased cortisol concentrations and production rates in ACTH-replete adult cancer survivors after cranial irradiation for nonpituitary brain tumors. J Clin Endocrinol Metab 2005;90:5217-25.

33. Fliers E, Klieverik LP, Kalsbeek A. Novel neural pathways for metabolic effects of thyroid hormone. Trends Endocrinol Metab 2010;21:230-6.

34. Alkemade A. Central and peripheral effects of thyroid hormone signalling in the control of energy metabolism. J Neuroendocrinol 2010 Jan;22(1):56-63.

35. Fliers E, Klieverik LP, Kalsbeek A. Novel neural pathways for metabolic effects of thyroid hormone. Trends Endocrinol Metab 2010 Apr;21(4):230-6.

36. Churilla JR, Fitzhugh EC. Total physical activity volume, physical activity intensity, and metabolic syndrome: 1999-2004 National Health and Nutrition Examination Survey. Metab Syndr Relat Disord 2012;10:70-6.

37. Rowlands AV, Eston RG. The measurement and interpretation of children's physical activity. Journal of Sports Science and Medicine 2007:270-76.

38. Ross R, Janssen I. Computed tomography and magnetic resonance imaging. Human Body Composition 2nd edition Heymsfield SB, Lohman TG, Wang Z, et al, editors 2005:89-108.

39. Radiation CotBEol. Health Effects of Exposure to Low Levels of Ionizing Radiation. BEIR V Washington, DC: National Academy Press 1990.

40. Shimizu Y, Kato H, Schull WJ. Studies of the mortality of A-bomb survivors. 9. Mortality, 1950-1985: Part 2. Cancer mortality based on the recently revised doses (DS86). Radiat Res 1990;121:120-41. 
41. Geenen MM, Bakker PJ, Kremer LC, Kastelein JJ, van Leeuwen FE. Increased prevalence of risk factors for cardiovascular disease in long-term survivors of acute lymphoblastic leukemia and Wilms tumor treated with radiotherapy. Pediatr Blood Cancer 2010;55:690-7.

42. van Dijk IW, Oldenburger F, Cardous-Ubbink MC, et al. Evaluation of late adverse events in long-term wilms' tumor survivors. Int J Radiat Oncol Biol Phys 2010;78:370-8.

43. Meacham LR, Chow EJ, Ness KK, et al. Cardiovascular risk factors in adult survivors of pediatric cancer-a report from the childhood cancer survivor study. Cancer Epidemiol Biomarkers Prev 2010;19:17081.

44. Moller DE, Flier JS. Insulin resistance--mechanisms, syndromes, and implications. N Engl J Med 1991;325:938-48.

45. Lippi G, Montagnana M, Franchini M, Favaloro EJ, Targher G. The paradoxical relationship between serum uric acid and cardiovascular disease. Clin Chim Acta 2008;392:1-7.

46. Guven A, Inanc F, Kilinc M, Ekerbicer H. Plasma homocysteine and lipoprotein (a) levels in Turkish patients with metabolic syndrome. Heart Vessels 2005;20:290-5.

47. Shlipak MG, Sarnak MJ, Katz R, et al. Cystatin C and the risk of death and cardiovascular events among elderly persons. N Engl J Med 2005;352:2049-60.

48. Martin SS, Qasim A, Reilly MP. Leptin resistance: a possible interface of inflammation and metabolism in obesity-related cardiovascular disease. J Am Coll Cardiol 2008;52:1201-10.

49. Im JA, Kim SH, Lee JW, Shim JY, Lee HR, Lee DC. Association between hypoadiponectinemia and cardiovascular risk factors in nonobese healthy adults. Metabolism 2006;55:1546-50.

50. Herder C, Karakas M, Koenig W. Biomarkers for the prediction of type 2 diabetes and cardiovascular disease. Clin Pharmacol Ther 2011;90:52-66.

51. Polak JF, Pencina MJ, Pencina KM, O’Donnell CJ, Wolf PA, D'Agostino RB, Sr. Carotid-wall intima-media thickness and cardiovascular events. N Engl J Med 2011;365:213-21.

52. Safar ME, Jankowski P. Central blood pressure and hypertension: role in cardiovascular risk assessment. Clin Sci (Lond) 2009;116:273-82.

53. Roman MJ, Devereux RB, Kizer JR, et al. Central pressure more strongly relates to vascular disease and outcome than does brachial pressure: the Strong Heart Study. Hypertension 2007;50:197-203.

54. Franklin SS, Khan SA, Wong ND, Larson MG, Levy D. Is pulse pressure useful in predicting risk for coronary heart Disease? The Framingham heart study. Circulation 1999;100:354-60.

55. Nijdam ME, Plantinga $\mathrm{Y}$, Hulsen $\mathrm{HT}$, et al. Pulse pressure amplification and risk of cardiovascular disease. Am J Hypertens 2008;21:388-92.

56. Moller DE, Kaufman KD. Metabolic syndrome: a clinical and molecular perspective. Annu Rev Med 2005;56:45-62.

57. Van Bortel LM, Struijker-Boudier HA, Safar ME. Pulse pressure, arterial stiffness, and drug treatment of hypertension. Hypertension 2001;38:914-21.

58. Rung J, Cauchi S, Albrechtsen A, et al. Genetic variant near IRS1 is associated with type 2 diabetes, insulin resistance and hyperinsulinemia. Nat Genet 2009;41:1110-5.

59. Zeggini E, Scott LJ, Saxena R, et al. Meta-analysis of genome-wide association data and large-scale replication identifies additional susceptibility loci for type 2 diabetes. Nat Genet 2008;40:638-45.

60. Levy D, Ehret GB, Rice K, et al. Genome-wide association study of blood pressure and hypertension. Nat Genet 2009;41:677-87.

61. Lindgren CM, Heid IM, Randall JC, et al. Genome-wide association scan meta-analysis identifies three Loci influencing adiposity and fat distribution. PLoS Genet 2009;5:e1000508. 




\section{Summary}

\section{Chapter 1}

Chapter 1 provides an overview of the epidemiology of childhood cancer and emphasizes the increased survival rates. The excess mortality and morbidity in childhood cancer survivors is highlighted and consequently, the relevance of assessing late effects in general and the metabolic syndrome in particular is described. Metabolic syndrome increases the risk for cardiovascular diseases. Furthermore, the pathophysiology of the metabolic syndrome is described. Finally, the aims and outline of the current thesis are presented.

\section{Chapter 2}

Literature concerning metabolic syndrome and its separate components in childhood cancer survivors is summarized in this chapter. Based upon previous studies of (components of) the metabolic syndrome, survivors of childhood cancer seem to be at increased risk. Especially the prevalence of obesity is increased in adult childhood cancer survivors, however, increased prevalence of insulin resistance, dyslipidemia and hypertension has also been reported. Possible explanations for this increased prevalence are hypothalamic or pituitary damage, thyroid or gonadal dysfunction, endothelial cell damage and reduced physical activity. In particular cranial irradiation, but also chemotherapeutic agents and radiation to the neck have been associated with an increased risk for metabolic syndrome or its components. Studies reporting the full content of the metabolic syndrome in large cohorts of childhood cancer survivors are scarce and have so far been focused on acute lymphoblastic leukemia (ALL) survivors.

\section{Chapter 3}

To assess the frequency of the metabolic syndrome in a heterogeneous group of childhood cancer survivors, we performed a retrospective single centre study in 500 adult long-term childhood cancer survivors. We found $13 \%$ of the survivors to have the metabolic syndrome. This seemed to be comparable with the prevalence of the metabolic syndrome in the normal population in the Netherlands (14\%). Comparable with previous findings, we observed the metabolic syndrome mainly in the subgroup of ALL survivors treated with cranial irradiation as compared with ALL survivors not treated with cranial irradiation ( $23 \%$ vs. $7 \%$ ). This increased prevalence was mainly due to increased frequency of hypertension and hypercholesterolemia. Furthermore, ALL survivors treated with cranial irradiation were more frequently overweight and had lower insulin like growth factor (IGF)-1 levels.

\section{Chapter 4}

As only scarce data had been available so far on this particular subgroup we focused in more detail on non-Hodgkin lymphoma (NHL) survivors in order to see whether NHL survivors are 
also at higher risk for endocrine late sequaelae. We found that body composition, bone mineral density and thyroid function were normal in NHL survivors, but observed low antimüllerian hormone (AMH) and low inhibin B levels in 15\% of adult females and $52 \%$ of adult males, respectively. Interestingly, in this pilot study we found cytarabin to be significantly associated with lower inhibin B levels in males, suggestive of a specific influence on gonadal damage, which had not been previously reported.

\section{Chapter 5}

The next subgroups we described consisted of survivors of nephroblastoma and neuroblastoma. Late effects after treatment in these survivors may be aggravated by the removal of the affected organ, as unilateral nephrectomy and adrenalectomy are often performed. It is unknown whether adrenal function after unilateral adrenalectomy declines over time by aging, especially in survivors who were also treated with chemotherapy or local radiotherapy. As increased mortality rates to date remain partly unexplained we hypothesized that adrenal insufficiency, or relative insufficiency during disease, contributes to this excess mortality later in life. We assessed adrenal function and its metabolic effects in nephroblastoma and neuroblastoma survivors and observed that all survivors, regardless of the presence of both or one adrenal gland(s), had adequate and thus not impaired adrenal function. Interestingly, survivors treated with unilateral adrenalectomy had relatively high basal cortisol and adrenocorticotropic hormone (ACTH) levels, indicating a higher central setpoint of the hypothalamic-pituitary-adrenal axis. Although the pathogenesis of this possible higher setpoint in adrenalectomized survivors is not clear yet, elevated cortisol and ACTH levels were associated with adverse lipids and insulin resistance, indicating that they influence the metabolic system in this subset of survivors.

\section{Chapter 6}

Another important confounder when measuring the metabolic syndrome is physical activity. Therefore, daily life physical activity in survivors of nephroblastoma and neuroblastoma was evaluated using the SQUASH-questionnaire. Male neuroblastoma survivors were less physically active than male control subjects, which was not due to determined cardiomyopathy or spinal deformities. Physical activity appeared to be related to both diagnosis and gender and is also known to be related to components of the metabolic syndrome. We therefore adjusted for physical activity in all studies involving nephroblastoma and neuroblastoma survivors.

\section{Chapter 7}

In this chapter we assessed the metabolic syndrome in nephroblastoma and neuroblastoma survivors. We observed an increased risk for developing components of the metabolic syndrome, especially after abdominal irradiation but also after chemotherapy. The results showed that radiotherapy involving both head and tail of the pancreas was associated with higher free fatty acids, triglyceride and glucose levels, indicating that pancreas irradiation may be an important 
denominator for future metabolic syndrome and subsequent cardiovascular disease. Likewise, abdominal irradiation involving the total liver seems to increase low density lipoproteincholesterol (LDL-C) levels, in contrast to irradiation involving only part of the liver. These results emphasize the need for more detailed studies involving larger cohorts and detailed radiation dosimetry, which will unravel the mechanisms behind the development of these late effects.

Although we found abdominally irradiated survivors already to be at higher risk for developing components of the metabolic syndrome than non-irradiated survivors, we found the prevalence of the metabolic syndrome in survivors treated with abdominal irradiation seems to be underestimated due to their dysmorphic abdominal area. Waist circumference, which is one of the components of the metabolic syndrome as defined by international and standardized definitions, was lower in irradiated survivors, however total percentage fat was significantly higher. Alternative markers for adiposity, such as total body fat, should therefore be included in future studies in order to get insight in the true prevalence of the metabolic syndrome after abdominal irradiation.

\section{Chapter 8}

In this chapter we described the study on preclinical atherosclerosis and combinations of biomarkers associated with the metabolic syndrome in nephroblastoma and neuroblastoma survivors and observed an increased risk for developing a risk profile for cardiovascular diseases. Nephroblastoma and neuroblastoma survivors, especially after abdominal irradiation, showed increased blood pressure and features of arterial stiffness. Additionally, survivors treated with abdominal radiotherapy showed risk profiles consisting of unfavourable lipids and adipocyte derived parameters. A history of nephrectomy was significantly associated with a risk profile consisting of high cystatin $\mathrm{C}$, uric acid, urea and homocystein, which are known to be associated with impaired renal function as well as cardiovascular disease. Furthermore, cumulative dose of actinomycin and cyclophosphamide seemed to determine increased arterial stiffness. Survivors of nephroblastoma and neuroblastoma thus show vascular alterations and risk profiles consisting of biomarkers, and together with the increased frequency of the components of the metabolic syndrome in this subgroup, as described in the previous chapter, thus are at increased risk for developing cardiovascular diseases.

\section{Chapter 9}

Development of the metabolic syndrome is, in the general population, known to be determined by environmental factors and by genetic make-up. We studied the genetic variation as determined by candidate single nucleotide polymorphisms (SNPs) that were identified in the general population. No association of the candidate SNPs (genes JAZF1, THADA, IRS1, MRSA, TFAP2B, ATP2B1) with diabetes, waist circumference and blood pressure was observed. Furthermore, none of the seven SNPs was associated with the complete picture of the metabolic syndrome. The results show that treatment factors, in particular cranial or abdominal radiotherapy, are a 
more dominant than genetic variation for the development of metabolic syndrome in childhood cancer survivors.

\section{Chapter 10}

In the general discussion, we discuss our findings in a broader context. Furthermore, general conclusions are provided and clinical implications are mentioned. Finally, suggestions for future research are made. 


\section{Nederlandse Samenvatting}

\section{Hoofdstuk 1}

Hoofdstuk 1 geeft een overzicht van de epidemiologie van kinderkanker en benadrukt de toegenomen overlevingskansen. De verhoogde mortaliteit en morbiditeit in overlevenden van kinderkanker wordt beschreven evenals de relevantie van het vaststellen van late effecten in het algemeen, en het metabool syndroom in het bijzonder. Metabool syndroom verhoogt het risico op hart- en vaatziekten. Verder wordt de pathofysiologie van het metabool syndroom beschreven en worden de doelen en de omlijning van het huidige proefschrift gepresenteerd.

\section{Hoofdstuk 2}

In dit hoofdstuk is de literatuur over het metabool syndroom en de afzonderlijke componenten in overlevenden van kinderkanker samengevat. Gebaseerd op eerder studies lijken overlevenden van kinderkanker een verhoogd risico te hebben op het metabool syndroom. Vooral de prevalentie van obesitas is verhoogd in overlevenden van kinderkanker, maar ook insulineresistentie, dyslipidemie en hypertensie worden vaker gerapporteerd. Mogelijke verklaringen voor deze toegenomen prevalentie zijn hypothalame of hypofysaire schade, dysfunctie van de schildklier of van de gonaden, endotheelcel schade en vermindere fysieke activiteit. Vooral craniële bestraling, maar ook chemotherapeutica en bestraling op de nek zijn geassocieerd met een verhoogd risico op het metabool syndroom of de losse componenten. Studies die het volledige spectrum van het metabool syndroom hebben bepaald in grote cohorten van overlevenden van kinderkanker zijn schaars en hebben zich tot nu hoofdzakelijk gericht op overlevenden van acute lymfatische leukemie (ALL).

\section{Hoofdstuk 3}

Retrospectief hebben we de frequentie van het metabool syndroom bepaald in een heterogene groep van 500 volwassenen die op de kinderleeftijd kanker hebben gehad, en die daarvoor destijds behandeld zijn in het Sophia Kinderziekenhuis. We stelden vast dat $13 \%$ van de overlevenden het metabool syndroom heeft. Dit is vergelijkbaar met de prevalentie van het metabool syndroom in de normale bevolking in Nederland (14\%). Het metabool syndroom komt vooral voor in de subgroep van ALL overlevenden behandeld met craniële bestraling, in vergelijking met de ALL overlevenden die niet behandeld waren met craniële bestraling ( $23 \%$ vs. $7 \%$ ). Deze verhoogde prevalentie werd hoofdzakelijk bepaald door een verhoogde frequentie van hypertensie en hypercholesterolemie. Bovendien hadden ALL overlevenden behandeld met craniële radiotherapie vaker overgewicht en hadden zij lagere insulin like growth factor-1 (IGF-1) spiegels. 


\section{Hoofdstuk 4}

Omdat er tot op heden weinig gegevens beschikbaar zijn over lange termijn effecten van behandeling voor non-Hodgkin lymfoom (NHL), hebben we ons gericht op deze subgroep. Hierbij hebben we gekeken naar endocriene late effecten. We vonden dat lichaamssamenstelling, botdichtheid en schildklierfunctie normaal waren in NHL overlevenden, echter, het antimüllerian hormoon (AMH) en inhibine $B$ waren verlaagd in respectievelijk 15\% van de volwassen vrouwen en $52 \%$ van de volwassenen mannen. In deze pilot-studie deden we tevens de interessante bevinding dat cytarabine, een chemotherapeuticum, was geassocieerd met lagere inhibine B waardes in mannen. Dit suggesteert dat cytarabine een specifieke invloed heeft op gonadale schade, wat nog niet eerder is gerapporteerd.

\section{Hoofdstuk 5}

De volgende subgroepen die we hebben beschreven zijn overlevenden van nefroblastoom en neuroblastoom. Doordat het aangedane orgaan is verwijderd, kunnen late effecten van behandeling in deze overlevenden zijn verergerd. Bij deze patienten wordt vaak een unilaterale nefrectomie (verwijdering van de nier) of adrenalectomie (verwijdering van de bijnier) uitgevoerd. Het is onbekend of de bijnierfunctie na unilaterale adrenalectomie afneemt wanneer patiënten ouder worden, vooral in overlevenden die ook behandeld werden met chemotherapie of lokale bestraling. Omdat de verhoogde mortaliteit tot op heden gedeeltelijk onverklaard is gebleven, hypothetiseerden wij dat bijnierinsufficiëntie, of relatieve insufficiëntie tijdens ziekte, bijdraagt aan deze verhoogde mortaliteit op latere leeftijd. We bepaalden de bijnierfunctie en de metabole effecten hiervan in nefroblastoom en neuroblastoom overlevenden en vonden dat alle overlevenden een adequate bijnierfunctie hadden, ongeacht er één of beide bijnieren waren verwijderd. Een interessante bevinding was dat overlevenden die een unilaterale adrenalectomie hadden ondergaan, relatief hoge basale cortisol en adrenocorticotroop hormoon (ACTH) waardes hadden. Dit wijst erop dat zij een hoger centraal setpoint van de hypothalame-hypofysairebijnier as hebben. Hoewel de pathogenese van dit mogelijk hogere setpoint nog onduidelijk is, bleken deze verhoogde cortisol en ACTH waardes weer geassocieerd te zijn met lipiden en insulineresistentie. Dit betekent dat cortisol en ACTH het metabole systeem in deze subgroep van overlevenden beïnvloeden.

\section{Hoofdstuk 6}

Een andere belangrijke confounder bij het bepalen van het metabool syndroom is fysieke activiteit. Daarom evalueerden wij fysieke activiteit in nefroblastoom en neuroblastoom overlevenden met behulp van de SQUASH-vragenlijst. Mannelijke neuroblastoom overlevenden waren minder fysiek actief dan mannelijke controlepersonen. Dit werd niet veroorzaakt door vastgestelde cardiomyopathie of door misvormingen van de wervelkolom. Fysieke activiteit bleek dus gerelateerd aan de diagnose en aan geslacht. Bovendien is het bekend dat fysieke 
activiteit gerelateerd is aan componenten van het metabool syndroom. We hebben daarom in alle nefroblastoom en neuroblastoom studies gecorrigeerd voor fysieke activiteit.

\section{Hoofdstuk 7}

In dit hoofdstuk hebben we de frequentie van het metabool syndroom bepaald in nefroblastoom en neuroblastoom overlevenden. We vonden een verhoogd risico op het ontwikkelen van componenten van het metabool syndroom, vooral na abdominale bestraling, maar ook na chemotherapie. De resultaten lieten zien dat radiotherapie toegepast op zowel het hoofd als de staart van de pancreas geassocieerd was met hogere vrije vetzuren, triglyceriden en glucose waardes. Dit betekent dat pancreasbestraling een belangrijke determinant van toekomstig metabool syndroom, en dus ook van hart- en vaatziekten kan zijn. Tevens vonden we dat abdominale bestraling op de totale lever, vergeleken met bestraling op alleen een deel van de lever, voor hogere "low density lipoprotein-cholesterol" (LDL-C) waardes lijkt te zorgen. Deze resultaten benadrukken dat meer gedetailleerde studies nodig zijn in grote cohorten en met gedetailleerde bestralingsdosimetrie.

Eerder vonden we dat overlevenden die bestraald waren op de buik een hoger risico hadden op het ontwikkelen van componenten van het metabool syndroom dan niet-bestraalde overlevenden. Nu blijkt ook dat de prevalentie van het metabool syndroom onderschat werd, doordat deze patiënten een misvormd abdominaal gebied hebben. De middelomtrek, één van de componenten van het metabool syndroom zoals gedefinieerd door internationale en gestandaardiseerde definities, was daardoor kleiner in bestraalde patiënten, terwijl het percentage totaal vet juist hoger was. Alternatieve markers voor adipositas, zoals het percentage totaal vet, zouden daarom geïncludeerd moeten worden in toekomstige studies. Zo kan er inzicht verkregen worden in de ware prevalentie van het metabool syndroom na abdominale bestraling.

\section{Hoofdstuk 8}

In dit hoofdstuk beschrijven we de studie naar preklinische atherosclerose en combinaties van biomarkers die geassocieerd zijn met het metabool syndroom in nefroblastoom en neuroblastoom overlevenden. We vonden dat vooral overlevenden die bestraald waren op het abdomen een verhoogde bloeddruk en arteriële stijfheid hadden. Bovendien hadden deze overlevenden risicoprofielen die bestonden uit lipiden en door vetcel-geproduceerde stoffen. Een nefrectomie in de voorgeschiedenis was significant geassocieerd met een risicoprofiel bestaande uit hoge cystatine $\mathrm{C}$, urinezuur, ureum en homocysteine waardes. Hiervan is bekend dat ze geassocieerd zijn met zowel verminderde nierfunctie als hart- en vaatziekten. Verder bleken de cumulatieve doseringen van zowel actinomycine als cyslophosphamide geassocieerd te zijn met arteriële stijfheid. Mensen die op de kinderleeftijd een nefroblastoom of neuroblastoom hebben gehad, hebben dus een ander risicoprofiel, gebaseerd op biomarkers en vasculaire veranderingen. Deze factoren zorgen er, in combinatie met de verhoogde frequentie van componenten van het 
metabool syndroom, voor dat de overlevenden van nefroblastoom en neuroblastoom hoger risico hebben op het ontwikkelen van hart- en vaatziekten.

\section{Hoofdstuk 9}

De ontwikkeling van het metabool syndroom wordt in de normale bevolking bepaald door omgevingsfactoren en het genetische profiel. We bestudeerden de genetische variatie, door middel van zogenaamde kandidaat "single nucleotide polymorphisms" (SNPs), die in de normale bevolking zijn geïdentificeerd. Er werd geen associatie gevonden tussen de kandidaat SNPs (gelokaliseerd in de genen JAZF1, THADA, IRS1, MRSA, TFAP2B, ATP2B1) en diabetes, middelomtrek en bloeddruk. Tevens werd er geen associatie gevonden tussen deze zeven SNPs en het metabool syndroom. De resultaten laten zien dat de behandeling, met name craniële en abdominale bestraling, een belangrijkere determinant is voor de ontwikkeling van metabool syndroom in overlevenden van kinderkanker, dan genetische variatie.

\section{Hoofdstuk 10}

In de algemene discussie bediscussiëren we onze bevindingen in een bredere context. Verder trekken we algemene conclusies en beschrijven we de klinische implicaties. We sluiten af met suggesties voor toekomstig onderzoek. 


\section{Affiliations co-authors}

Author

Auke Beishuizen,

MD, PhD

Karin Blijdorp,

MD

Patric J.D. Delhanty,

PhD

Annelies Hartman,

PhD

Marry M. van den Heuvel-Eibrink, MD, PhD

\section{Martin T. Huisman}

Frank H. de Jong,

PhD

Robert de Jonge,

PhD

Aart-Jan van der Lelij,

MD, PhD

Caspar W.N. Looman,

MSc

Monique P.M. de Maat,

PhD

Francesco U.S. Mattace-Raso, MD, PhD

\section{Affiliation}

Department of Pediatric Oncology/Hematology, Erasmus MC - Sophia Children's Hospital, Rotterdam, the Netherlands

Department of Medicine, section Endocrinology, Erasmus University Medical Centre, Rotterdam, the Netherlands

Department of Pediatric Oncology/Hematology, Erasmus MC - Sophia Children's Hospital, Rotterdam, the Netherlands

Department of Medicine, section Endocrinology, Erasmus University Medical Center Rotterdam, the Netherlands

Department of Pediatric Physiotherapy, Erasmus MC Sophia Children's Hospital, Rotterdam, the Netherlands Department of Pediatric Oncology/Hematology, Erasmus MC - Sophia Children's Hospital, Rotterdam, the Netherlands

Department of Medicine, section Endocrinology, Erasmus University Medical Center Rotterdam, the Netherlands

Department of Medicine, section Endocrinology, Erasmus University Medical Centre, Rotterdam, the Netherlands

Department of Clinical Chemistry, Erasmus University Medical Centre Rotterdam, the Netherlands Department of Medicine, section Endocrinology, Erasmus University Medical Centre, Rotterdam, the Netherlands

Department of Public Health, Erasmus University Medical Centre Rotterdam, the Netherlands Department of Hematology, Erasmus University Medical Centre Rotterdam, the Netherlands

Department of Internal Medicine, Section of Geriatric Medicine, Erasmus University Medical Centre Rotterdam, the Netherlands 
Sebastian J.C.M.M. Neggers, MD, PhD

Max M. van Noesel, MD, PhD

Rob Pieters,

MD, MSc, PhD

Hein Raat,

MD, PhD

Caroline M. van Rij, MD, PhD

André G. Uitterlinden, MD, PhD

Andrica C. H. de Vries, MD

Mark Wijnen

Lizet te Winkel, MD
Department of Medicine, section Endocrinology, Erasmus University Medical Centre, Rotterdam, the Netherlands

Department of Pediatric Oncology/Hematology,

Erasmus MC - Sophia Children's Hospital, Rotterdam, the Netherlands

Department of Pediatric Oncology/Hematology,

Erasmus MC - Sophia Children's Hospital, Rotterdam, the Netherlands

Department of Pediatric Oncology/Hematology,

Erasmus MC - Sophia Children's Hospital, Rotterdam, the Netherlands

Department of Public Health, Erasmus University

Medical Centre Rotterdam, the Netherlands

Department of Radiation Oncology, Erasmus University

Medical Centre Rotterdam, the Netherlands

Department of Internal Medicine, Erasmus University

Medical Centre Rotterdam, the Netherlands

Department of Pediatric Oncology/Hematology,

Erasmus MC - Sophia Children's Hospital, Rotterdam, the Netherlands

Department of Pediatric Oncology/Hematology,

Erasmus MC - Sophia Children's Hospital, Rotterdam, the Netherlands

Department of Pediatric Oncology/Hematology,

Erasmus MC - Sophia Children's Hospital, Rotterdam, the Netherlands 


\section{List of abbreviations}

AACE

ACTH

AHA/NHLBI

ALL

$\mathrm{AMH}$

BCCSS

BMAD-LS

$B M C$

BMD-LS

BMD-TB

$\mathrm{BMI}$

$\mathrm{BP}$

CBG

CCSS

CRT

CT

CV

DC

DCOG

DXA

EGIR

FFA

FPG

$\mathrm{FSH}$

fT4

$\mathrm{GH}$

GWAS

HDL-C

HMW

HOMA

HPA

hsCRP
Association of American Endocrinologists

adrenocorticotrophin hormone

American Heart Association/National Heart, Lung and Blood Institute

acute lymphoblastic leukemia

anti-müllerian hormone

British Childhood Cancer Survivor Study

bone mineral apparent density of the lumbar spine

bone mineral content

bone mineral density of the lumbar spine

bone mineral density of the total body

body mass index

blood pressure

cortisol binding globulin

Childhood Cancer Survivor Study

cranial radiotherapy

computed tomography

coefficients of variation

distensibility coefficient

Dutch Childhood Oncology Group

dual-energy X-ray absorptiometry

European Group for the study of Insulin Resistance

free fatty acids

fasting plasma glucose

follicle stimulating hormone

free thyroxin

growth hormone

genome wide association study

high-density lipoprotein-cholesterol

high molecular weight

homeostatic model assessment

hypothalamic-pituitary-adrenal

high sensitivity C-reactive protein
Chapter 


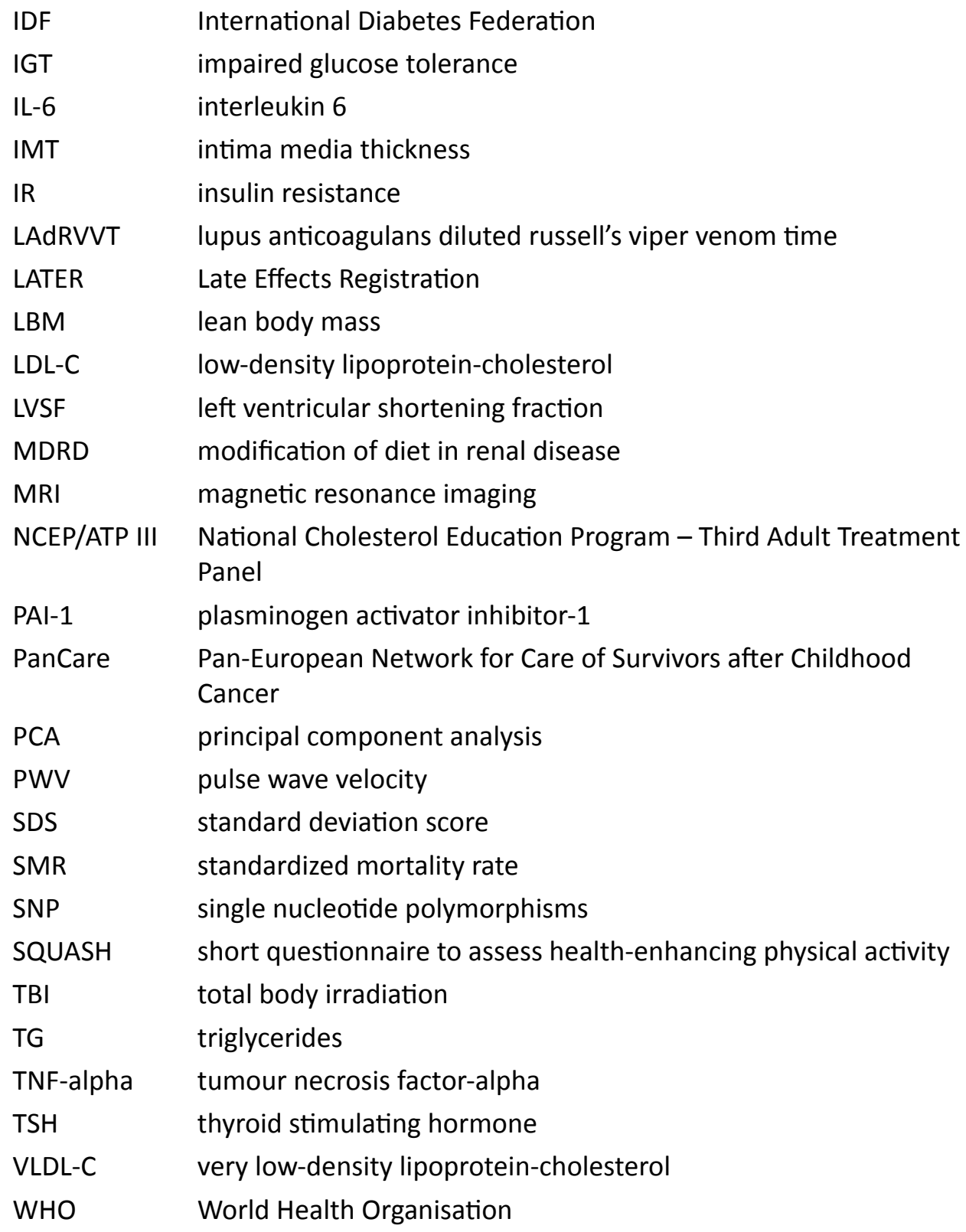




\section{Dankwoord}

Mijn proefschrift is af! Ik heb er de afgelopen jaren met veel plezier aan gewerkt, maar toch voelt het erg goed dat het nu af is. Tijdens mijn promotie heb ik ontzettend veel mensen ontmoet die, allemaal op hun eigen manier, veel voor mij hebben betekend. Dit is een mooi moment om daar bij stil te staan.

Allereerst wil ik alle patiënten en controlepersonen bedanken die hebben deelgenomen aan het onderzoek. Ik vond het heel bijzonder om een hele onderzoeksdag met jullie door te brengen en heb dit met veel plezier gedaan. Zonder jullie was dit proefschrift er niet geweest!

Mijn co-promotoren, Dr. M.M. van den Heuvel-Eibrink en Dr. S.J.C.M.M. Neggers. Marry, een paar jaar geleden kwam ik als student voor mijn afstudeeronderzoek bij jou en nu, een paar jaar later, sta ik hier. Ik wil je ontzettend bedanken voor je vertrouwen in mij, je altijd snelle reacties op mijn manuscripten en emails, je goede adviezen en samenwerking en ook je attentheid. Sebastian, wat fijn om een co-promotor te hebben die tevens in advies over de laatste iPhone apps en het beste parfum voorziet! Maar vooral natuurlijk heel erg bedankt voor de samenwerking, je wetenschappelijke input en het relativeren en motiveren de afgelopen jaren. Mijn promotor, Prof. dr. R. Pieters, beste Rob, bedankt voor de mogelijkheid om te kunnen promoveren op jouw afdeling. We hebben elkaar niet heel frequent gesproken de afgelopen jaren, maar je heldere visie en nuchtere commentaar heb ik altijd zeer gewaardeerd.

Prof.dr. A.P.N. Themmen, Prof.dr. A.J. van der Lelij en Prof.dr. J.J. van Binsbergen, hartelijk dank voor het lezen en beoordelen van mijn proefschrift.

Prof.dr. A.J. van der Heijden, Prof.dr.Ir. F.E. van Leeuwen en Prof.dr. H.N. Caron, veel dank voor het plaatsnemen in de promotiecommissie.

Dr. F.U.S. Mattace-Raso, beste Francesco, dank voor het aanleren van de vasculaire metingen zodat ik deze zelfstandig kon uitvoeren, en voor het meedenken over de analyses en het manuscript. Ir. C.W.N. Looman, dank voor uw deskundige statistische advies. Prof. H. Raat, dank voor het meedenken met de projecten en voor uw kritische, maar enthousiaste commentaar op de manuscripten. Patric Delhanty en Martin Huisman, heel erg bedankt voor de snelle uitvoering van en het meedenken over de leptin en adiponectin bepalingen en het manuscript. Dr. A. Beishuizen, Dr. M.M. van Noesel. Prof.dr. F.H. de Jong, Dr. R. de Jonge, Prof.dr. M.P.M. de Maat, Dr. C.M. van Rij, Prof. A.G. Uitterlinden, hartelijk dank voor jullie bijdrage en het tot stand komen van dit proefschrift. 
Drs. F.G.A.J. Hakvoort-Cammel en dr. G. van der Linden, door jullie ben ik eigenlijk geïntroduceerd en geënthousiasmeerd op en door de LATER-poli, dank jullie wel hiervoor.

Er waren ontzettend veel mensen betrokken bij de uitvoering van de studies die beschreven zijn in de laatste hoofdstukken. Allereerst Judith van Eck, dank je wel voor de fijne samenwerking tijdens alle vroege ochtenden waarop we de Synacthen-testen afnamen (en het weekend doornamen). Sjaan, Marianne en Joke, dank dat jullie altijd konden bijspringen wanneer dit nodig was. Kirsten, dank voor het inplannen van de vele telefonische afspraken. Serieta, Marjolein en Eveline, bedankt voor het meedenken en inplannen van alle patiënten voor de vasculaire metingen. Monique de Waart, Sjef van Asten en Ramazan Buyukcelik, dank voor jullie toegankelijkheid en het uitvoeren van de AKC, hemostase en SNP-bepalingen. Dames van de radiotherapieafdeling, hartelijk dank voor het opzoeken van de lastig terug te vinden statussen. Jeanine, Jacqueline en Anita, dank jullie wel voor alles de afgelopen jaren en met name de afgelopen maanden!

De vaste leden van de immer-groeiende QCAT-groep: Manita, Marjo, Andrica, Annelies, Lizet, Karin, Wendy, Wing, Lidewij, dank voor jullie aanwezigheid tijdens mijn promotie! Wat was het fijn om de afgelopen jaren met jullie te kunnen sparren over onderzoek, maar juist ook vaak over niet-onderzoek gerelateerde zaken. Ik wens jullie allemaal heel veel succes toe met jullie werk en onderzoek, en natuurlijk veel geluk! Manita, extra veel dank voor al jouw ondersteunend werk de afgelopen jaren. Karin, jou wil ik ook speciaal bedanken voor alles wat je de afgelopen jaren voor mij hebt gedaan: van het overnemen van mijn studie toen ik geveld was door de Pfeiffer, tot het ophalen van mijn posters om 5 uur's ochtends, en niet te vergeten de samenwerking voor het AML-manuscript. Wat fijn om op zo'n collega te kunnen bouwen!

Alle studenten die (rondom) de QCAT-groep onderzoek hebben gedaan of doen, bedankt voor jullie samenwerking en veel succes. Alle andere onderzoekers van de afdeling kinderoncologie/ hematologie, we zijn inmiddels met te veel om op te noemen; ik wens jullie allemaal heel veel succes met jullie onderzoek en verdere carriére.

Mijn allereerste echt vaste kamergenoten: Daan, Emile en Gerthe. De periode in het Chalet was toch wel de meest memorabele. Wat een goede combi van wetenschappelijke discussies, het opzetten van een paprikakwekerij, ijskoffies in de zon, en vooral heel veel lol! Ik ben heel blij dat we inmiddels al aan de reünie - deel 6 toe zijn en hoop dat er nog vele gaan volgen.

Lieve Gerthe, 3 maanden geleden was ik jouw paranimf en het kon natuurlijk niet anders dan dat jij nu de mijne bent! Wat hebben we veel met elkaar mee gemaakt en wat kennen we elkaar goed. Ik ben blij met zo'n slimme, lieve paranimf die ook nog eens alles over het metabool syndroom weet! 
Sandra, mijn andere paranimf, ook jou wil ik speciaal bedanken voor de laatste jaren. Wat was het fijn om met jou de kamer te delen, altijd vrolijk en vol bemoedigende en relativerende adviezen die altijd op het juiste moment kwamen. Dank je wel!

Mijn kamergenootjes van Sp-4478, Sjoerd, Nienke D, Nienke V, Sandra, Suzanne, Esther, Alexandra en Erik, het was misschien een beetje druk maar vooral ook heel gezellig!

Daarna door naar de Z-flat met ook Yvonne, Charlotte, Laura, Nynke, Yuen en Carlijn. Mede door jullie allemaal was het nooit een straf om naar het werk te gaan!

En dan nog alle andere Sophia onderzoekers die mijn promotietraject tot een fantastische tijd hebben gemaakt. Dankzij jullie heb ik ontzettend veel mooie herinneringen aan alle borrels, nieuwjaarsdiners, de weekendjes weg en het ski-weekend. Ik wens jullie allemaal veel geluk en succes met jullie verdere carrière!

Speciale dank aan Eefje, Marjolein, Anne Loes en Annemiek: voor alle cola-light breaks, de etentjes, biertjes \& bitterballen, de Sophia-hockeywedstrijden, de gedeelde onderzoeks- en andere frustraties!

Lieve Elske, als geen ander begreep jij de afgelopen jaren mijn promotiegerelateerde hoogte- en dieptepunten. Van congres naar festival, van repeated measurements naar een tripje New York, en van telefoongesprekken vol intellectuele ideeën naar babybezoek en jouw bruiloft! Ik ben heel blij dat ik jou heb leren kennen!

Mijn vriendinnen: Ineke, Renske, Stella, Stefanie, Joyce, Rachel, Gerbrich (ook als collega!), Rosanne, Louise, Thamar, Carly en Eva; dank jullie wel voor jullie belangstelling en steun. We denken wel eens dat ons studentenleven het hoogtepunt was, maar ik weet zeker dat het alleen nog maar beter gaat worden! Ik ben ontzettend blij met jullie en hoop dat we tot we grijze oude besjes zijn weekendjes weg blijven gaan. Speciale dank aan len, nu al 14 jaar mijn beste maatje. Dankjewel dat je er altijd voor mij bent! En ook speciale dank aan Rens, wat ben ik blij met onze vriendschap en wat was (en ben!) je belangrijk voor mij geweest de afgelopen jaren! Julia, Tessa, Sally, Marloes B en Marloes v/d H, dank voor al jullie belangstelling, steun en gezelligheid!

Mijn schoonfamilie, wat fijn dat jullie mij zo hartelijk ontvangen hebben en altijd belangstellend zijn, al wonen we niet bij elkaar om de hoek! An\&Martin, vroeger al mijn lievelingsoom en tante en nu nog steeds: Dank jullie wel dat jullie altijd voor mij klaarstaan en geïnteresseerd zijn in mij. Mijn fantastische broers, Mark en Erik, wat ben ik blij met deze mannen in mijn leven! Mark, altijd geïnteresseerd en trots, en Erik, altijd enthousiast en zelfs als proefpersoon in mijn onderzoek betrokken. Ik ben ook ontzettend trots op jullie! Lieve Marloes, wat fijn om zo'n geweldige schoonzus te hebben, altijd attent en vol leuke plannen. 
Jos en Irene, mijn geweldige papa en mama. Pap en mam, ik weet niet waar ik moet beginnen met jullie bedanken. Dank jullie wel voor jullie onvoorwaardelijke liefde en voor al het vertrouwen dat jullie mij altijd gegeven hebben. Mam, dankzij jou heeft dit proefschrift een prachtige omslag, die behalve veel andere parallellen misschien nog wel de mooiste parallel toont met alle mogelijkheden die jullie mij altijd hebben geschonken. Ik kan me geen betere ouders wensen dan jullie!

Lieve Sascha, dank je wel dat je er altijd voor me bent! Je maakt mijn leven compleet. Op naar een geweldige toekomst samen! 


\section{Curriculum vitae}

Marjolein van Waas was born in Vlaardingen on March $17^{\text {th }}, 1985$. She passed her secondary school exam (gymnasium) at "Scholengemeenschap Spieringshoek" in Schiedam in 2003. In the same year she started her medical training at the Medical Faculty of the Erasmus University Rotterdam. During her study she spent five weeks in Brazil where she did an internship in obstetrics/gynaecology at the Hospital Nossa Senhora da Conceição, Tubarão. Furthermore, she performed research at the department of Pediatric Oncology/Hematology (supervisors drs. F.G.A.J Hakvoort-Cammel and dr. M.M. van den Heuvel-Eibrink). She graduated on the theoretical part of her medical training in September 2007 and after six months of travelling in Australia and New Zealand she started her PhD project in April 2008 at the department of Pediatric Oncology/ Hematology of the Erasmus MC - Sophia Children's Hospital (supervisors Prof.dr. R. Pieters, dr. M.M. van den Heuvel-Eibrink, dr. S.J.C.M.M. Neggers). The research performed during this period is presented in this thesis. Furthermore, she received funding from the Scholten-Cordes foundation and from the department of Pediatric Oncology/Hematology, enabling her to enroll in the masterprogram of the Netherlands Health Institutes for Sciences (NIHES) in Rotterdam. In August 2010 she received her master of science in "Clinical Epidemiology". In May 2012 she restarted her Medicine study and started with her internships. 



\section{List of publications}

1. van Waas M, Neggers SJ, van der Lely AJ, Pieters R, van den Heuvel-Eibrink MM. The metabolic syndrome in adult survivors of childhood cancer, a review. J Pediatr Hematol Oncol 2010; 32:171-9.

2. van Waas M, Neggers SJ, Pieters R, van den Heuvel-Eibrink MM. Components of the metabolic syndrome in 500 adult long-term survivors of childhood cancer. Ann Oncol 2010; 21:1121-6.

3. van Waas M, Neggers SJ, Te Winkel ML, Beishuizen A, Pieters R, van den Heuvel-Eibrink MM. Endocrine late sequelae in long-term survivors of childhood non-Hodgkin lymphoma. Ann Oncol 2011;23:1626-32.

4. van Waas M, Neggers SJ, van Eck JP, van Noesel MM, van der Lely AJ, de Jong FH, Pieters R, van den Heuvel-Eibrink MM. Adrenal function in adult long-term survivors of nephroblastoma and neuroblastoma. Eur J Cancer 2012;48:1159-66.

5. van Waas M, Wijnen M, Hartman A, de Vries AC, Pieters R, Neggers SJ, van den HeuvelEibrink MM. Daily life physical activity in adult long-term nephroblastoma and neuroblastoma survivors. Submitted.

6. van Waas M, Neggers SJ, Raat H, van Rij CM, Pieters R, van den Heuvel-Eibrink MM. Abdominal irradiation: An important determinant of metabolic syndrome after nephroblastoma and neuroblastoma Submitted.

7. van Waas M, Neggers SJ, Looman CW, Raat H, de Maat MP, de Jonge R, Delhanty PJ, Huisman M, Mattace-Raso FU, Pieters R, van den Heuvel-Eibrink MM. Cardiovascular risk factors in adult very long-term survivors of nephroblastoma and neuroblastoma. Submitted.

8. van Waas M, Neggers SJ, Uitterlinden AG, Blijdorp K, Pieters R, van den Heuvel-Eibrink MM, Treatment factors but not genetic variation determine metabolic syndrome in childhood cancer survivors. Submitted.

9. Klap BC, te Winkel ML, van Waas M, Neggers SJ, Boot AM, Pieters R, van den Heuvel-Eibrink MM. Sequential measurement of bone mineral density identifies ongoing recovery of detrimental bone mass in very long term adult survivors of childhood cancer. Submitted.

10. Blijdorp K, van Waas M, van der Lely AJ, Pieters R, van den Heuvel-Eibrink MM, Neggers SJ. Endocrine sequelae and metabolic syndrome in adult long-term survivors of childhood acute myeloid leukemia. Submitted. 



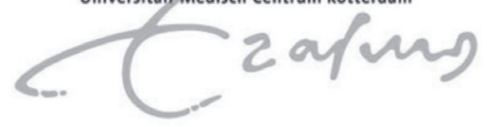

\section{Summary of PhD training}

Erasmus MC Department: Pediatric Oncology/Hematology

Research School: Molecular Medicine Postgraduate School

PhD period: 1 april $2008-1$ april 2012

Master of Science in Clinical Epidemiology 2008 - 2010

Promotor: Prof.dr. R. Pieters

Co-promotors: Dr. M.M. van den Heuvel-Eibrink, Dr. S.J.C.M.M. Neggers

\begin{tabular}{lc}
\hline General academic courses & Year \\
\hline Basiscursus regelgeving en organisatie voor klinisch onderzoekers & 2008 \\
Biomedical English Writing and Communication & 2009 \\
Molecular Diagnostics - MolMed & 2009 \\
Single Nucleotide Polymorphisms (SNP) course - MolMed & 2011 \\
\hline
\end{tabular}

Research skills

Master of Science in Clinical Epidemiology

$2008-2010$

NIHES

Including elective/in depth courses:

- Planning and Evaluation of Screening

- Genetic Analysis in Clinical Research

- Analysis of Growth Data

- Repeated Measurements

Weekly research meeting Pediatric Oncology/Hematology

2008-2012

Weekly research meeting Quality of Life and Toxicity of Care Working group

2008-2012

\section{(Inter)national conferences}

$-10^{\text {th }}$ International Conference on Long-Term Complications of Treatment of

2008

Children \& Adolescents for Cancer, Niagara-on-the-Lake, Canada

- European Symposium on Late Complications after Childhood Cancer, Edinburgh, 2009 Scotland (oral presentation)

- American Society of Hematology (ASH) - 51 $1^{\text {st }}$ Annual Meeting, New Orleans,

2009

U.S.A. (poster presentation)

- Novel insights in adipose cell functions - Ipsen Foundation, Paris, France

$-11^{\text {th }}$ International Conference on Long-Term Complications of Treatment of

Children and Adolescents for Cancer, Williamsburg, U.S.A. (oral presentation)

$-42^{\text {nd }}$ Congress of the International Society of Paediatric Oncology (SIOP), Boston, 
- European Symposium on Late Complications after Childhood Cancer 2011, Amsterdam, the Netherlands (2 poster presentations)

$-43^{\text {rd }}$ Congress of the International Society of Paediatric Oncology (SIOP),

Auckland, New Zealand (poster presentation)

- European Congress of Endocrinology, Florence, Italy (poster presentation)

$-12^{\text {th }}$ International Conference on Long-Term Complications of Treatment of

Children and Adolescents for Cancer, Williamsburg, U.S.A. (2 poster presentations)

- ENDO 2012 - $94^{\text {th }}$ Annual Meeting \& Expo, Houston, U.S.A. (oral presentation)

$-44^{\text {th }}$ Congress of the International Society of Paediatric Oncology (SIOP), London, United Kingdom (oral presentation)

\section{Symposia \& seminars}

$-1^{\text {st }}$ National DCOG 'LATER' Conference, University Medical Centre Groningen, 2008 the Netherlands

- Annual Pediatric Research day, Erasmus MC, Rotterdam, the Netherlands 2008, 2009

- Annual PhD day, Erasmus MC, Rotterdam, the Netherlands

- Annual Pediatric Oncology Symposium, Erasmus MC, Rotterdam, the Netherlands 2008-2010

- Meeting Integraal Kanker Centrum Rotterdam (IKR), Rotterdam, the Netherlands

2009 (oral presentation)

- Symposium 'Early growth, infant feeding and long-term risk for metabolic and cardiovascular disease', Erasmus MC, Rotterdam, the Netherlands

- Minisymposium: Metabolomics of the obese, Erasmus MC, Rotterdam, the Netherlands

- Diabetes symposium, Erasmus MC, Rotterdam, the Netherlands

$-2^{\text {nd }}$ National DCOG 'LATER' Conference “Guidelines and late effects research for childhood cancer survivors", Erasmus MC, Rotterdam, the Netherlands

- "SKION promovendidag", Utrecht, the Netherlands (oral presentation)

- Dutch Hematology Conference, Papendal, the Netherlands (oral presentation)

- "SKION promovendidag", Utrecht, the Netherlands (oral presentation)

\section{Grants}

- Grant for Master of Science in Clinical Epidemiology, awarded by the 2008 Scholten-Cordes Foundation

- Travel grant for the 11th International Conference on Long-Term Complications 2010 of Treatment of Children and Adolescents for Cancer, awarded by Erasmus MC Trustfonds

- Travel grant for ENDO $2012-94^{\text {th }}$ Annual Meeting \& Expo, awarded by Erasmus MC Trustfonds

- Travel grant for ENDO 2012 - 94th Annual Meeting \& Expo, awarded by 


\section{Supervising Master's thesis}

- Mrs. B. C. Klap, student Medicine, Leiden University Medical Centre, "Survivors

2010-2011 of childhood cancer: peak bone mass"

- Mr. M. Wijnen, student Medicine, Erasmus University Rotterdam, "Physical activity in adult long-term survivors of nephroblastoma and neuroblastoma"

- Mrs. L.S. Hoek, student Medicine, Leiden University Medical Centre, "Evaluation of liver toxicity using the APRI method in long-term survivors of childhood cancer"

\section{Other}




